SAND96-2019

Unlimited ReIease

Printed August 1996

Distribution

Category UC-600

\title{
Environmental Measurement-While-Drilling- Gamma Ray Spectrometer (EMWD-GRS) System Technology Demonstration Plan for Use at the Savannah River Site F-Area Retention Basin
}

\author{
Cecelia V. Williams \\ Environmental Restoration Technologies \\ Grant J. Lockwood \\ Materiais Radiation Science \\ Randy A. Nomann \\ Geothermal Rosearch \\ Sandia National Laboratories \\ Albuquexque, New Mexico $87185-5800$ \\ Rajph D. Gruebel \\ Tech Reps, Inc. \\ Albuquerque, New Mexico 87110
}

\begin{abstract}
The Environmental Measurement-While-Drilling-Gamma Ray Spectrometer (EMWD-GRS) system represents an innovative blend of new and existing technology that provides the capability of producing real-time-environmental and drillbit data during drilling operations. This demonstration plan presents information on the EMWD-GRS technology, demonstration design, Cs-137 contamination at the Savannah River Site F+Area Retention Basin, responsibilities of demonstration participants, and the policies and procedures for the demonstration to be conducted at the Savannah River Site F-Area Retention Basin.

The EMWD-GRS technology demonstration will consist of continanusly monitoring for gamma-ratiation contamivation whtle drilling two bojizossal borsholes befow the backfilled retention basin. These boreholes will pass near previously sampled vertical borehole locations whers concenirations of contaminant levels are known. Contaminant levels contintrously recorded by the EMWD-GRS system duritg drillting witl be compared to contaritiant levels previously detennined through quantitative laboratory analysis of soil samples.
\end{abstract}




\section{Acknowledgment}

This Envitonmental Measurenent-While-Drilling-Gamma Ray Spectrometer (EMWDGRS) system technology demonstration was funded by the Office of Science and Technoloby (OST) under U.S. Department of Energy (U.S. DOE) contract number DE-AC04-94AL85000.

The authors wist to acknowledge the contributions made by the following individuals in support of this demonstration:

Scott McMullen (DOE/Savannah River Site) for his confidence in the EMWD project and his vision in facilitating its demonstration at the Savannah River Site. This denonstration would not have taken place without his direct personal involvement.

Pete Zionkowski (Westinghouse Savannah River Company) for his administrative, logistics, and technical support.

Ahmet Suer (Westinghouse Savannah River Company) for his dedication to planning the demonstration, his technical support, and his tenacity in seeing that the demonstration oceusted.

Kevin Kuelske, William Pardee, James Jordon, and Edward Leibfarth (Westinghouse Savannah River Company) for their technical review of this demonstration plan. 


\section{DISCLAIMER}

Portions of this document may be illegible in electronic image products. Images are produced from the best available original document. 



\section{Contents}

ACKNOWLEDGMENT

CONTENTS

EXECUTTVE SUMMARY vii

ABBREVIATIONS AND ACRONYMS

1.0 INTRODUCTION

1.1 Techrology Dexponstration Purpose and Objectives 1

1,2 Predemorstration Arangements 1

1.3 Demonstration Sito 1

1.4 Demonstration Schedule $\quad$. 2

1.5 Demonstratjon Participants 2

2.0 DEMONSTRATION RESPONSIBILITIES AND COMMUNICATION

2.1 Demonstration Participants 3

2.2 Responsibilities 5

2,3 Communication 6

2.4 Personnel Locations 6

3.0 PREDEMONSTRATION ACTIVITIES 7

3.1 Site Selection 7

3.2 Predemonstration Site Characterization 7

4.0 TECHNOLOGY DESCRIPTION

4.1 Technology Capabilities 9

4.2 The EMWD-GRS System 9

4.2.1 Basic Systen. Operation

4.2.2 System Adaptability 9

4.2.3 Drilling Platform $\quad 12$

4.2.4 Down-hole Components 12

4.2.5 Up-hole Components 12

4,2,6 Cable Deployment System 13

4.2.7 Data Collection System

5.0 DEMONSTRATION SITE DESCRIPIION

S.1 Site Narne and Location 15

5.2 Site History . 15

5.3 Sit Characteristics 15

5.3.1 General Savanuah River Site Geology

5.3.2 F-Area Retention Basin Site Soils 19

5.3.3 Site Hydrogeology 19

5.3.4 Site Contaminants and Distribution 19

6.0 DRILLING AND DATA COLLECTION PLAN 27

6.1 Overview of Drilling and Evaluation Operations 27

6.2 Communications, Documentation, Logistics, and Equipment 27

6.3 Data Collection Procedures 28

63.1 Drilling Lacations 28

6.3.2 EMWD-GRS Data Collection 28

6.3.3 Decontamination and Disposal of Generated Waste 28

6.3.4 Schedule $\quad 30$

7.0 QUALITY ASSURANCE PROJECT PLAN 31

7.1 Purpose and Scope 31 
7.2 Quality Assurance Responsibilities 31

7.3 Data Quality Parameters 31

7.3.1 Representativeness

7.3 .2 Completeress 32

7.3 .3 Comparability $\quad 32$

7.3.4 Aceuracy

7.3.5 Precision 33

7.3.6 Probability of False-Positive 33

7.4 Calibration Procedures, Quality Control Checks, and Corrective Action 34

7.4.1 Calibration Procedures $\quad 34$

7.4.2 Soil Sample Analysis $\quad 34$

7.5 Data Reduction, Validation, and Reporting 34

7.5.1 Data Reduction $\quad 35$

7.5.2 Data Validation $\quad 35$

7.5.3 Data Reporting $\quad 35$

7.6 Data Quality Indicators

7.7 Performance and System Audits $\quad 35$

7.7.1 Performance Audit

7.7.2 On-Site System Audits 36

7.8 Quality Assurance Reports to Project Manager $\quad 36$

7.8.1 Status Reports $\quad 36$

7.8.2 Audit Reports $\quad 36$

7.9 Corrective Action 36

8.0 DATA MANAGEMENT AND ANALYSIS

8.1 EMWD-GRS Reading and Location Identification 37

8.2 Data Aralysis and Comparison with Provious Site Characterization Data 37

9.0 HEALTH AND SAFETY PLAN 39

9.1 Site Specific Health and Safey Plan (HASP)

9.2 Health and Safety Ptan Enforcement

I0.0 DELIVERABLES 4]

11.0 REFERENCES

Appendices

APPENDIX A Background Soil Aralytical results F-A rea Retention Basin - A-1 APPENDIX B Quantitative Laboratory Analysis of Soil Samples collected from Sampling . Locations FRB-05, 06, 07, 08, and 19 at the SRS F-Area Retention Basin ---B-1

APPENDIX C Environmental Measarement-While-Drilling-Gamma Ray Spectrometer

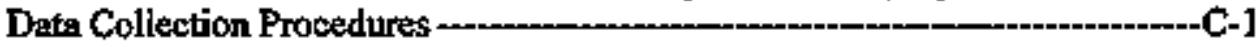

APPENDIX D Environmental Measurement-While-Drilling-Gamma Ray Spectrometer

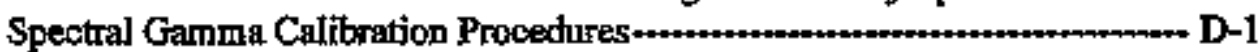

APPENDIXE Site Specific Health and Safety Plan for the EMWD-GRS System

Demonstration at the SRS F Area Retention Basis- 


\section{FIGURES}

Figure 1. Demonstration organizational structure. -

Figure 2. Soil sampling sites in and around the F-Area Retention Basin _.

Figure 3. Garnma Ray Spectrometer Environmental Measurement-While-Drilling System component placement on typical directional boring rig. 10

Figure 4. The Environmental Measurement-While-Drilling process using a coaxial spool.-. 11

Figure 5. Location of Savannah River Site F-Area Retention Basin, - 16

Figure 6. Topographic map of F-Area Retention Basin and surrounding area.---.--.-__- 17

Figure 7. General stratigraphic units underlying the Savannah River Site. 18

Figure 8. Hydrostratigraphic anits underlying the Savannab River \$ite,__________

Figure 9. Location of groundwater monitoring wells in the vicinity of the F-Area Retention Basin.

Figure 10. Potentiometric surface of the water table (Aquifer Unit IIB) at the F-Area Retention Basin.

Figure 11. Concentration ( $\mathrm{pCi} / \mathrm{g}$ ) of Cs-137 relative to depth on sampling locations FRB-05, 06, 07, 08, and 19 within the SRS F-Area Retention Basin._.........._. 24

Figure 12. Concentration ( $\mathrm{pCi} / \mathrm{g}$ ) of $\mathrm{Sr}-90$ relative to depth at sampling locations FRB-0S, 06, 07, 08, and 19 within the SRS F-Area Retention Basin.- 25

Figure 13. Locations for drilling continuously monitored daylight-to-daylight boreholes at the Savannah River Site F-Area Retention Basin. 29

\section{TABLES}

Table 1. Demonstration participant information

Table 2. Chemicats of Concern at the SRS F-Area Retention Basin- 
This Page Intentionally Left Blank

1 


\section{EXECUTIVE SUMMARY}

\section{Introduction}

The Environmental Measurement-While-Drilling-Gamma Ray Spectrometer (EMWDGRS) system represents an innovative blend of new and existing technology that produces the capability of providing real-time environmental and drill bit data during drilling operations. These real-time measurements provide technical data for field screening (i.e., "steering" the drill bit in or out of contaminated zones). There are also time, cost, and safety advantages to using the EMWD GRS system's field sereening approach: (1) data on the nature of contamination are available in minutes, as opposed to weeks or months for ofisite confirmatory analysis; (2) strbstantial cost savings result by mininizing the number of samples required for off-site confirmatory analyses; and (3) worker safety is enhanced through the minimization of waste generated during drilling and by quickly alerting field personne] to potentially hazardous conditions; and (4) the amount of investigation derived waste (IDW) is recuced.

\section{Technology Description}

The EMWD-GRS system is compatible with a directional driling technique that uses minimai drilling fluids and generates little or no secondary waste. The down hole sensor is located behind the drill bit and is linked by a high-speed data transuission system to a computer at the surface. Windows'n-based software, developed by Sandia National Laboratories, is used for data display and storage. During drilling operations, data on the nature and extent of contanination are collected. Instant access to the data provides information for on-site decisions regarding drilling and sampling strategies.

Down-hole components of the EMWD-GRS systen being demonstrated consist of a gamma ray spectrometer, a muitichannel analyzer, a 900 V power supply, a signal conditioning and transmitter board, and a coil containing coaxial cable for transmitting data to the surface. To protect them from the drilling environment, down-hole components are contained within O-ringsealed aluminum tubes. The up-hole system consists of a personal computer, a battery pack/coil, a pickup coil, and a receiver. During drilling, the GRS system monitors (1) gamma radiation, (2) the $+12 \mathrm{~V}$ and $-12 \mathrm{~V}$ required at the dowr-hole signal conditioning and transmitter board, (3) the up-hole battery voltage as measured down-hole, and (4) two temperatures associated with the detector and instrumentation. The systen design incorporates datz quality assurance techniques to ensure data reliability.

The EMWD system can provide real-time data on an 8 differential/single analog multiplexer and on any number of digital channels. Sampling speed from the analog channels can reach $100 \mathrm{kHz}$. For the EMWD-GRS system, threo digital channols are used. Readings are taken at a rate of 20 per second. The telemetry system is programinable firtiware that can easily support many different dati formats and additional data channels. The currently used format (Digital FM Bi-phase, 4800 baud) provides excellent noise rejection. A Sandia National Laboratories (SNL) -designed receiver removes FM carrier noise, generates data clock, and buffers data to be used by an IBM or compatible personal computer. A $28 \mathrm{~V}$ rechargeable battery pack can supply down-hole instrumentation power for more than 18 hours of irilling. The battery pack remains topside for easy maintenance. 


\section{Demonstration Location}

The EMWD-GRS system will be demonstrated tat the Savannah River Site (SRS) F-Area Retention Basin near Aiken, South Carolina. Tho basin measures $200 \mathrm{ft}(61 \mathrm{~m})$ in length, $120 \mathrm{ft}$ (36.5 $\mathrm{m}$ ) in width and $6.9 \mathrm{ft}(2 \mathrm{~m})$ in depth, with a total volume capacity of approximately 6,128 cubic yards $\left(4,685 \mathrm{~m}^{3}\right)$. This basin was constructed as an unlined, tefaporary container for potentially contaminated cooling water associated with the chemical separations process and stomn sewer drainage from the F-Area Tank Farm. Contamination of this basin came from contaminated cooling water as well as various spills or overflows at the basin (WSRC, 1995). The basin was in active use from 1955 until 1972. In 1972, it was replaced by a lined retention basin located to the west of the site. In 1978, the F-Area Retention Basin was excavated, backfilled with soil, and covered with grass. The site was evaluated through soil sampling in early 1979. The major radionuclides present included strontiun (Sr)-89/90 and cesium (Cs)-137. Sampling results indicated maximum Cs-137 concentrations of $1,410 \mathrm{pCi} / \mathrm{g}$ in the berm and 430 $\mathrm{pCi} / \mathrm{g}$ it the basin floor. Maximum Sr- $89 / 90$ concentrations in the basin berm were $1,000 \mathrm{pCi} / \mathrm{g}$ (WSRC, 1994).

\section{EMWD-GRS System Demonstration}

Phase I of the demonstsation at tie F-Area Retention Basin will determine Cs-137 background conditions for the site. Background conditions will be deternined by dritling one horizontal borehole, approximately $50 \mathrm{ft}(15 \mathrm{~m}$ ) in length at a depth between 10 and $15 \mathrm{ft}$ ( 3 and $4.5 \mathrm{~m}$ ), at an adjacent radiologically "clean" test site. After backeround conditions are deternined, the system will be demonstrated in the previously characterized F-Area Retention Basin.

Phase II of the EMWD-GRS system demonstration at the F-Area Retention Basin will consist of monitoring environmental conditions in real-time while drilling two boreholes daylight-to-daylight. These holes will pass near sample locations FRB-05, 06, 07, 08, and 19 where values of contaminant levels are known. Contaninant levels continuously recorded by the EMWD-GRS systera during drilling will be compared to contaminant levels previously determined throagh quantitative laboratory analysis of soil samples collected at locations FRB$05,06,07,08$, and 19 . 


\section{ABBREVIATIONS AND ACRONYMS}

\begin{tabular}{|c|c|}
\hline $\begin{array}{l}\text { AC } \\
\mathbf{b p s}\end{array}$ & $\begin{array}{l}\text { alternating current } \\
\text { bits per second }\end{array}$ \\
\hline CERCLA & $\begin{array}{l}\text { Comprehensive Environmental Response, } \\
\text { Compensation, and Liability Aet }\end{array}$ \\
\hline $\mathrm{COC}$ & contaminants of concern \\
\hline Co & cobalt \\
\hline Cs & cesium \\
\hline $\mathrm{DC}$ & direct current \\
\hline DOE & U.S. Department of Energy \\
\hline EMWD & Environmental Measurement - While-Drilling \\
\hline EPA & U.S. Environmental Protection Agency \\
\hline FM & freçuency modulated \\
\hline FRB & F-Area Retention Basin \\
\hline $\mathrm{ft}$ & feet \\
\hline GRS & Gamma Ray Spectrometer \\
\hline HASP & health and safety plan \\
\hline HSO & health and safety officer \\
\hline IBM & International Business Machines \\
\hline IC & integrated circuit \\
\hline IDW & investigation derived waste \\
\hline K & potassiunn \\
\hline$\cdot$ & kilo \\
\hline $\mathbf{k H z}$ & kilohertz \\
\hline $\mathbf{k P a}$ & kitopascals \\
\hline $\mathbf{m}$ & meter(s) \\
\hline ma & milliampere(s) \\
\hline mtn & millimeter(s) \\
\hline msl & mean sea level \\
\hline NRZ & non-retarm to zero \\
\hline OD & outside diameter \\
\hline OST & Office of Science and Technology \\
\hline
\end{tabular}


PC

$\mathrm{pCi} / \mathrm{g}$

psi

QA.

QA/QC

QC

QAPP

$\mathrm{RCO}$

RF

RI

SNL

SR.

Sr

SRS

V

WSRC personal computer

picocuries per gram

pounds per square inch

quality assurance

quality assurance/quality control

quality control

quality assurance project plan

radiological control operations

radio frequency

remedial investigation

Sandia National Laboratories

Savaninah River

strontium

Savannah River Site

$\operatorname{volt}(\mathrm{s})$

Westinghouse Savannah River Company 


\subsection{INTRODUCTION}

The Environmental Measurement-While-Drilling- Gamma Ray Spectrometer (EMWDGRS) system represents an innovative blend of new and existing technology to produce the capability of providing real-fime environmental and drill bit data during drilling operations. These real-time measurements provide techmical data for field screening (i.e, ability to "steet" the drill bit in or out of contaminated zones). There are also time, cost, and safety advantages to using the field screening approach of the EMWD-GRS system: (1) data on the nature of contamination is available in minutes, as opposed to weeks or months for offsite confirmatory analysis; (2) stbstantial cost savings result by minimizing the number of samples required for off-site confimatory analyses; and (3) worker safety is enhanced through the minimization of waste generated during drilling and by quickly alerting field personnel to potentially hazardous conditions; and (4) reduction in investigation derived waste (IDW).

\subsection{Technology Demonstration Purpose and Objactives}

During development, the EMWD-GRS system was tested at the U.S. Department of Energy (DOE) radiation test facility in Grants, New Mexico, and at the directional boring test site owned by Charles Machine Works in Perry, Oklahoma. Demonstration at the Savannah River Sito (SRS) F-Area Retention Basin will provide BMWD-GRS system performance data under field conditions at a previously characterized "hot" site. The system's real time measurements of radjonuclide contaminant presence will be compared to tadionuclide contaminant levels previously determined through soil sampling and kaboratory analysis.

\subsection{Predemonstration Arrangements}

During October of 1995, a representative of Westinghouse Savannah River Company (WSRC) contacted Sandia National Laboratories (SNL), the EMWD-GRS system technology developer, about dentonstrating the system at the SRS. During November 1995, representatives of SNL and Westinghouse Savannah River Company met and developed a draft agreement to demonstrate the system at the \$RS near Aiken, South Carolina.

\subsection{Demonstration Site}

The site selected for the demonstration is the proviously characterized F-Area Retention Basin located outside and south of the F-Area perimeter fence and east of bujlding 281-8F of the SRS. The basin is approximately $200 \mathrm{ft}(61 \mathrm{~m})$ long, $120 \mathrm{ft}(36.5 \mathrm{~m})$ wide, and 6.9 feet ( $2 \mathrm{~m})$ deep and has a total volume capacity of approximately 6,128 cubic yards $\left(4,685 \mathrm{~m}^{3}\right)$. This basin was constructed as an unlined, temporary container for potentially contaminated cooling water from the chemical separations process and stom sewer drainage from the F-Area Tank Farm. When contamination was detected in cooling water, the water was diverted to the retention basin or F-Area seepage basins. Additional contamination of this basin came from various spills or overflows at the basin (WSRC, 1995). The bssin was in active use from 1955 until 1972, when it was replaced by a lined retention basin located to the west of the site. In 1978, the F-Area Retention Basin was excavated, backfilled with soil, and covered with grass. At that time, a process sewer line which extended from the basin to a diversion box located approximately 500 feet $(152 \mathrm{~m})$ north of the basin was abandoned. The site was evaluated through soit sampling in 
early 1979. The major radionuclides present included Strontium (Sr)-89/90 and Cosium (Cs)137. Sampling results indicated maximur Cs- 137 concentrations of $1,410 \mathrm{pCi} / \mathrm{g}$ in the berm and $430 \mathrm{pCi} / \mathrm{g}$ in the basin floor. Maximum Sr-89/90 concentrations in the bastin berm were 1,000 $\mathrm{pCi} / \mathrm{g}$ (WSRC, 1995).

\subsection{Demonstration Schedule}

The agreement formally authorizing the demonstration was sabmitted to the Department of Energy-Savaruah River (DOE-SR) on March 8, 1996. The demonstration is scheduled for April 22-26, 1996.

\subsection{Demonstration Participants}

Demonstration participants include DOE-SR, DOE-Albuquerque, Westinghouse Savannah Rjver Company, and Sandia National Laboratories.

The demonstration is being conducted by SNL at the DOE-SR facility near Aiken, South Carolina. Site access and associated logistics will be performed by Westimghouse Savaniah River Company. Health-and-safety officer oversight will be performed by SNL personnel. 


\subsection{DEMONSTRATION RESPONSIBILITIES AND COMMUNICATION}

This section identifies the organizations involved in this demonstration and describes the ptimary responsibilities of each organization. The section also details the methods and frequency of communication to be used to coordinate the demionstration.

\subsection{Demonstration Participants}

This demonstration is being conducted at the DOE-SR facility near Aiken, South Carolina. Site access and associated logistics will be performed by Westinghouse Savannah River Company. Heath-and-safety-offlicer oversight will be performed by SlNL personnel. The organizational structure for the derionstration showing lines of communication is provided in Figure I.

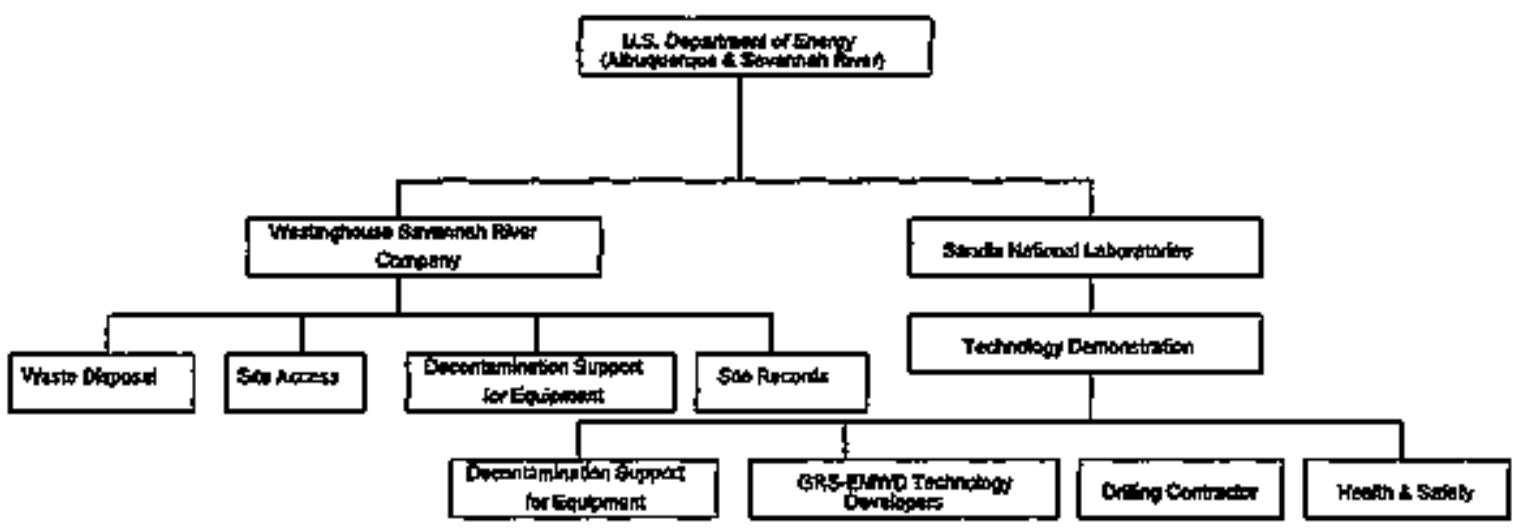

Figure 1. Demonstration organizational structure. 
SNL and Westingtrouse Savannah River Company personnel participating in this demonstration are Iisted in Table 1 . The specific responsibilities of SNL and Westinghouse Savannab River Cornpany are discussed in Section 2.2.

Table 1. Demonstration Parficipant Information

\begin{tabular}{|c|c|c|c|c|c|c|}
\hline Name & Anllation & Reqponstbilky & $\begin{array}{l}\text { Sailliwe } \\
\text { Address }\end{array}$ & $\begin{array}{l}\text { Telephonet } \\
\text { Number }\end{array}$ & Fax Nothor & Entailaddress \\
\hline $\begin{array}{l}\text { Cectilav. } \\
\text { Willianss }\end{array}$ & $\mathrm{S} \mathrm{NiL}^{2}$ & $\begin{array}{l}\text { Projtct Lowder/ } \\
\text { QA Managert } \\
\text { Site Safty officer }\end{array}$ & $\begin{array}{l}\text { P.0. Box 5800 } \\
\text { Albuquerque, } \\
\text { Nu } 87185-0719\end{array}$ & (505) $844-572$ & $(505) 844-0543$ & cruilitgsandiagov \\
\hline $\begin{array}{l}\text { Gtant J, } \\
\text { Lockwootd }\end{array}$ & SNL & Fiteld Team Lesater & $\begin{array}{l}\text { P.d. Bux 5800 } \\
\text { Albuqutaque, } \\
\text { NM } 87185-0719\end{array}$ & $(505) 845+7007$ & (S0S) $84 \overline{-724}$ & glock phsandiagor \\
\hline $\begin{array}{l}\text { Randy A } \\
\text { Nouman }\end{array}$ & SNL & $\begin{array}{l}\text { Technology } \\
\text { Operator }\end{array}$ & $\begin{array}{l}\text { P.0. Box } 5800 \\
\text { Albuquerque, } \\
\text { NM } 87185-0719\end{array}$ & $(505) 845-9675$ & $(505) 844.5952$ & tamamagsandlagov \\
\hline Johar Ha्वll & $\begin{array}{l}\text { Genera } \\
\text { Cosp-Biteh- } \\
\text { Witeh of of } \\
\text { Goorita }\end{array}$ & $\begin{array}{l}\text { Diflliog } \\
\text { Contractiot }\end{array}$ & $\begin{array}{l}\text { 4891 Clark } \\
\text { Howell Hwy } \\
\text { College } \\
\text { Pat, GA } 30349\end{array}$ & (404)761-0619 & $(404) 761-4095$ & None \\
\hline Personutid & SNL & $\begin{array}{l}\text { Decottingination } \\
\text { Support for } \\
\text { Equipment }\end{array}$ & WA & W/A & NA & $\sqrt{N A}$ \\
\hline $\begin{array}{l}\text { Pele } \\
\text { Zironkonadki }\end{array}$ & WSRC: & $\begin{array}{l}\text { Tecbinics } \\
\text { Demonstralions } \\
\text { Manager }\end{array}$ & 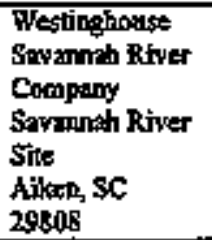 & $(903) 952-6487$ & $(869) 952.6538$ & $\begin{array}{l}\text { peterzionkowski@sr. } \\
\text { gov at hubsmtp }\end{array}$ \\
\hline Afinet Suer & WSRC & Technforal Contagt & $\begin{array}{l}\text { Westioghouse } \\
\text { Ssuminah Rutwer } \\
\text { Company } \\
\text { Swarensh River } \\
\text { Stte } \\
\text { Aiken, SC } \\
2980.8\end{array}$ & $(808) 952.8305$ & $(809) 952-6538$ & $\begin{array}{l}\text { Thimet.suergats gov ai } \\
\text { hubeunt }\end{array}$ \\
\hline $\begin{array}{l}\text { Keviin } \\
\text { Kuelske }\end{array}$ & WASRC & $\begin{array}{l}\text { Fachlicy Project } \\
\text { Maptger }\end{array}$ & $\begin{array}{l}\text { Westinghoust } \\
\text { Straanah River } \\
\text { Company } \\
\text { Savannah River } \\
\text { Stto } \\
\text { Alken, SC } \\
29 \% 08\end{array}$ & $(\$ 03) 952-6516$ & (903) 952-6538 & 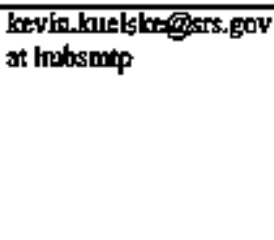 \\
\hline Persantal & WSTRC & $\begin{array}{l}\text { Radiological } \\
\text { Control } \\
\text { Operations (RCO) } \\
\text { Covtratse and } \\
\text { Suppont }\end{array}$ & NA & $\overline{\mathrm{NA}}$ & $\bar{N} / \mathbf{A}$ & $\mathbf{W h}$ \\
\hline Perstomntal & WSRC & $\begin{array}{l}\text { Decontaquatation } \\
\text { Support for } \\
\text { Equipmeat }\end{array}$ & NA & $\overline{\mathbf{N A}}$ & TWA & $\sqrt{W A}$ \\
\hline
\end{tabular}




\subsection{Responsibilities}

Sandia National Laboratories personnel have the following responsibilities for the demonstration:

- designing and preparing the demonstration plan;

- developing a quality assurance project plan (QAPP) (Section 7 of the demonstration plan):

- detailing procedures for using the technology;

- providing demonstration technology;

- providing drilling subcontractor for performance of the demonstration (decontamination of difller's equipment is the responsibility of the drilling contuactor);

- operating and monitoring the EMWD-GRS system during the demonstration;

- documenting the experimental methodojogy and operation of the EMWD-GRS system during the demonstration;

- reducing and interpreting data, as required;

- providing site health \& safexy officer, and

- preparing a final report detailing demonstration resplts.

Westinghouse Savannah River Compeny personnel have the following responsibilities for the demonstration:

- providing site access;

- providing radiological control operations ( $\mathrm{RCO}$ ) coverage and support;

- providing a health and safety plan (HASP) (Section 9 of the demonstration plan) for the demonstration activities;

- providing health and safezy information;

- providing site characterization information;

- providing site characterization confinatiory analysis data;

- providing other logistical infonmation and support needed to coordinate access to the sito for the field portion of the demonstration; and

- Providing decontanination support for equiputent and disposal of investigation derived waste (II) W). 


\subsection{Communication}

Both SNL and Westinghouse Savannah River Company have primary points of contact. These points of contact are:

SNI

Cecelia V. Williams $\quad$ Phone: (505) 844-5722;

Fax: (505) 844-0543

Westinghouse SaxannahRiver Company

Pete Zionkowski

Phone: (803) 952-6487 or (803) 952-6538

Ahmet Suer - Technical Contact Phone: (803) 952-8306;

Fax: (803) $952-6538$

\subsection{Personnel Locations}

Personnel actively participating on-site in the field portion of the EMWD-GRS technology demonstration are frotn SNL, Westimghouse Savannah River Company, and Geneva Corporation-Ditch-Witch ${ }^{\mathrm{mM}}$ of Georgia.

All SNL personnel are tocated in Albuquerque, New Mexico. All Westinghouse Savannah River Company personnel are located in Aiken, South Carolina The drilling contractor, Geneva Corporation-Ditch-Witch ${ }^{\text {TM }}$ of Georgia, is located in College Park, Georgia. 


\subsection{PREDEMONSTRATION ACTIVITIES}

\subsection{Site Selection}

The site was selected besed on site availability, access, and previous site characterization that documented the presence of radionuclide-contaminated soits.

\subsection{Predemonstration Site Characterization}

The F-Area Retention Basin site was initially characterized in 1979 by surveying 53 soil cores in the field for the presence of radionuclide species. Ten of the cores were chosen for further evaluation in the laboratory because of high levels of radioactivity (Scott et al., 1987 and WSRC, 1994a). Strbsequent investigations include (WSRC 1994a):

- Phase I investigation (1993-1994)-collection and analysis of soil samples to determine potential contaminants of concern (COC) and their approximate concentrations in the soils and backftil;

- Phase 1A investigation (July 1994)-collection and analysis of background soil and groundwater samples from the groundwater aguifer in the vicinity of the basin and implementation of a video and radiological servey of the internal surfaces of the associated abandoned process line;

- Phase II Remedial Investigation (RI) (in progress)-to define the extent and magritude of tadiological substance rejease to environmental media surnounding the F-Area Retention Basin and associated process line (WSRC, 1994a). This investigation has documented the radionuclide contamination within the basin through soi] sampling and laboratory analysis (Figure 2) (WSRC, 1995).

The two daylight-to-daylight boreholes to be drilled and monitored for radiontaclides in real-time will pass near sampled locations FRB-05, 06, 07, and 08. The borehole that passes near FRB-05 and 06 will also pass near FRB-19. 


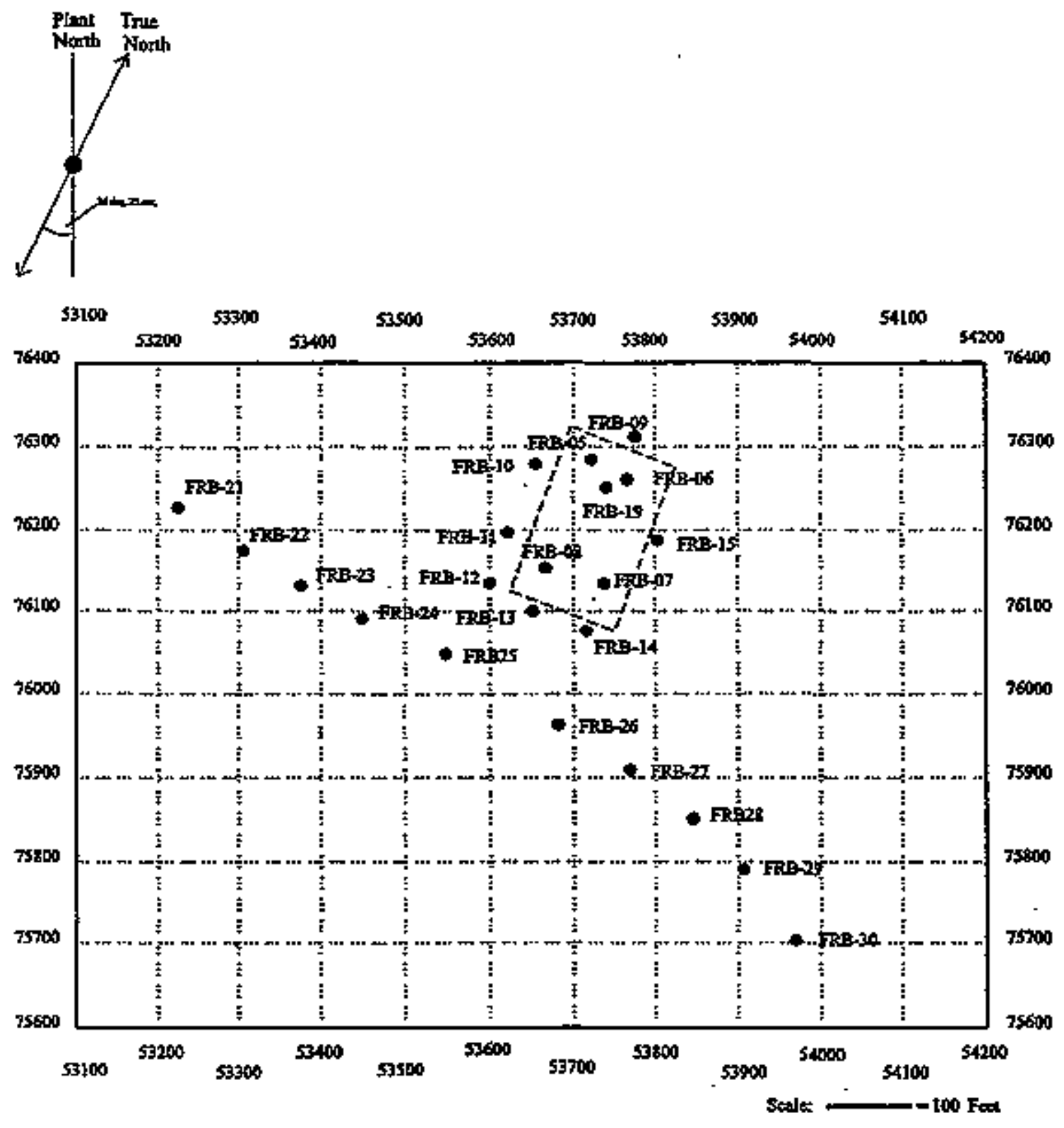

Figure 2. Soil sampling sites in and around the F-Area Retention Basin (WSRC, 1995). 


\subsection{TECHNOLOGY DESCRIPTION}

Sampling during environmental drilling is essential for complete characterization of the spatial distribution and migration of near surface contaninants. However, analysis of these samples is not only expensive, but also can take weeks or months when sent to an off-site laboratory. In contrast, the environmental measurenent-while-dritling (EMWD) screening capability saves money and time by quickly distinguishing befween contaminated and unconteminated areas. Real-time measurements provided by a EMWD -Gamma Ray Spectrometer (GRS) systenn woutd enable on-the-spot decisions about sampling strategies and would enhance worker safety. The system also provides the added flexibility of being able to "steer" the drill bit into or out of hazardons zones.

\subsection{Technology Capabilities}

The EMWD-GRS system is compatible with a directional drilling technique that uses minimal ditling fluids and generates little or no secondary waste. The down-hole sensors are located behind the drill bit and are linked by a high-speed data transmission system to a computer at the surface. Sandia-developed, Windowsth-based software is used for data display and storage. As drilling is conducted, data are collected on the nature and extent of contamination. Data are instantly accessible for on-the-spot decisions about dritling and sampling strategies. The system also has the capability of being able to "steer" the drill bit into or out of hazardous zones.

\subsection{The EMWD-GRS System}

\subsubsection{Basic System Operation}

The system is comprised of four parts: a computer, magnetic pickup coil and receiver, battery pack and magnetic coil, and a down-hole electronics package. The electronies package, complete with a gamma ray speetrometer, multichanmel analyzer, and coaxial coil, is located inside the drill rod next to the drill bit. The coil provides both direct current (DC) power and alternating current (AC) signal paths between the surface and the down-hole electronics package. Figure 3 shows the instrumentation mounting locations on a typical drill rig.

Figure 4 shows drilling steps. As the drill string is lengthened by adding drill rod, the coaxial cable is unspooled. The unspooled cable is attached to the battery pack and coil. The latter are mounted on the rotating drill pipe which extends behind the bydraulic head. The coil couples the AC signal between the rotating drill pipe and the stationary coil and receiver, which are mounted on the drilling plationm. The receiver converts the AC signal into a serial bit stream. A computer equipped with a telemetry sertal card receives the data and jisplays downhole measarements in real time.

\subsubsection{System Adaptability}

The electronics package, located near the drill bit, is easily adaptable to different sensors or date formats. Adaptability is gained by using an Actel 1020B programmable logic 


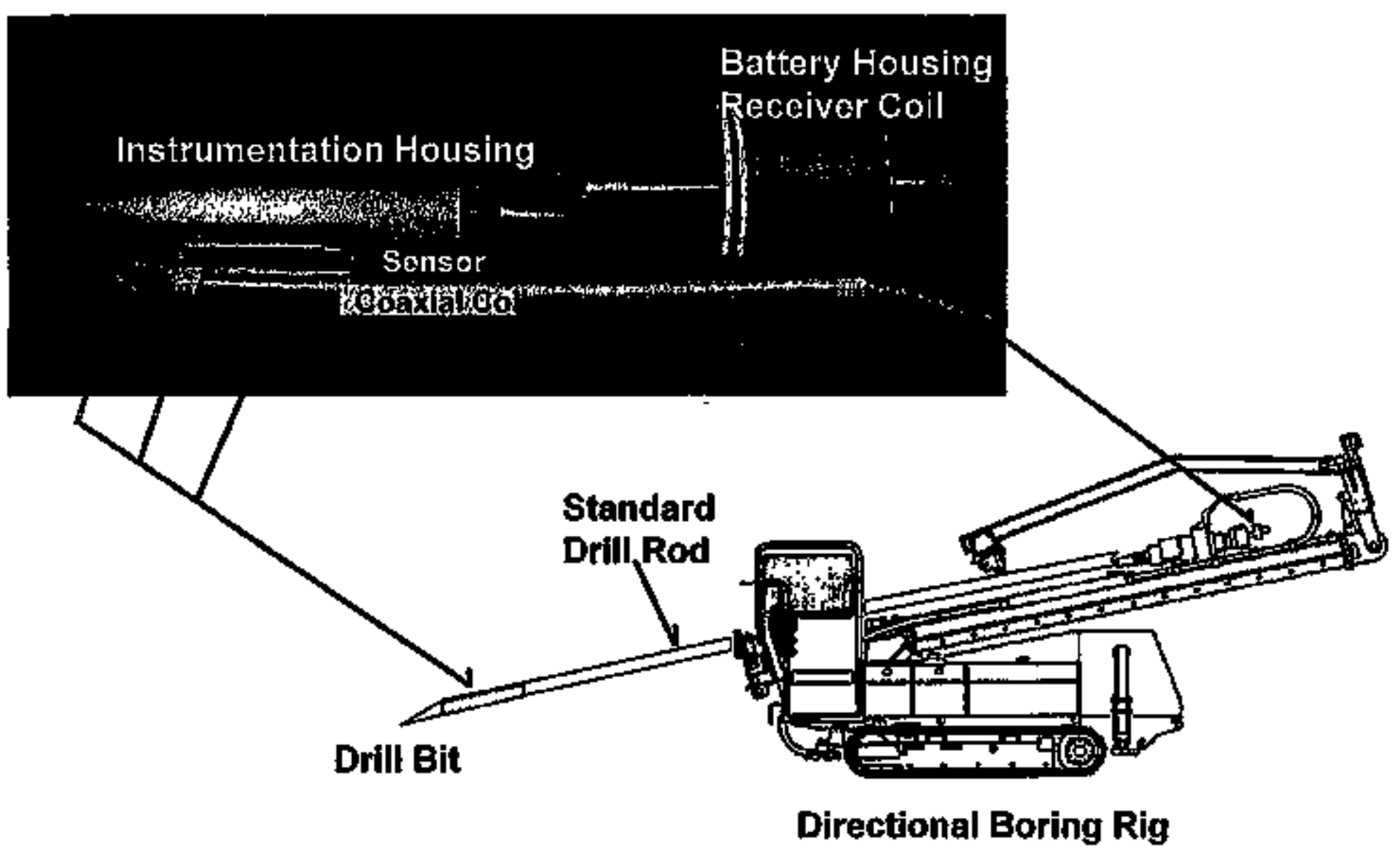

Figure 3. EMWD-GRS system component placentent on typical directional boring rig. 

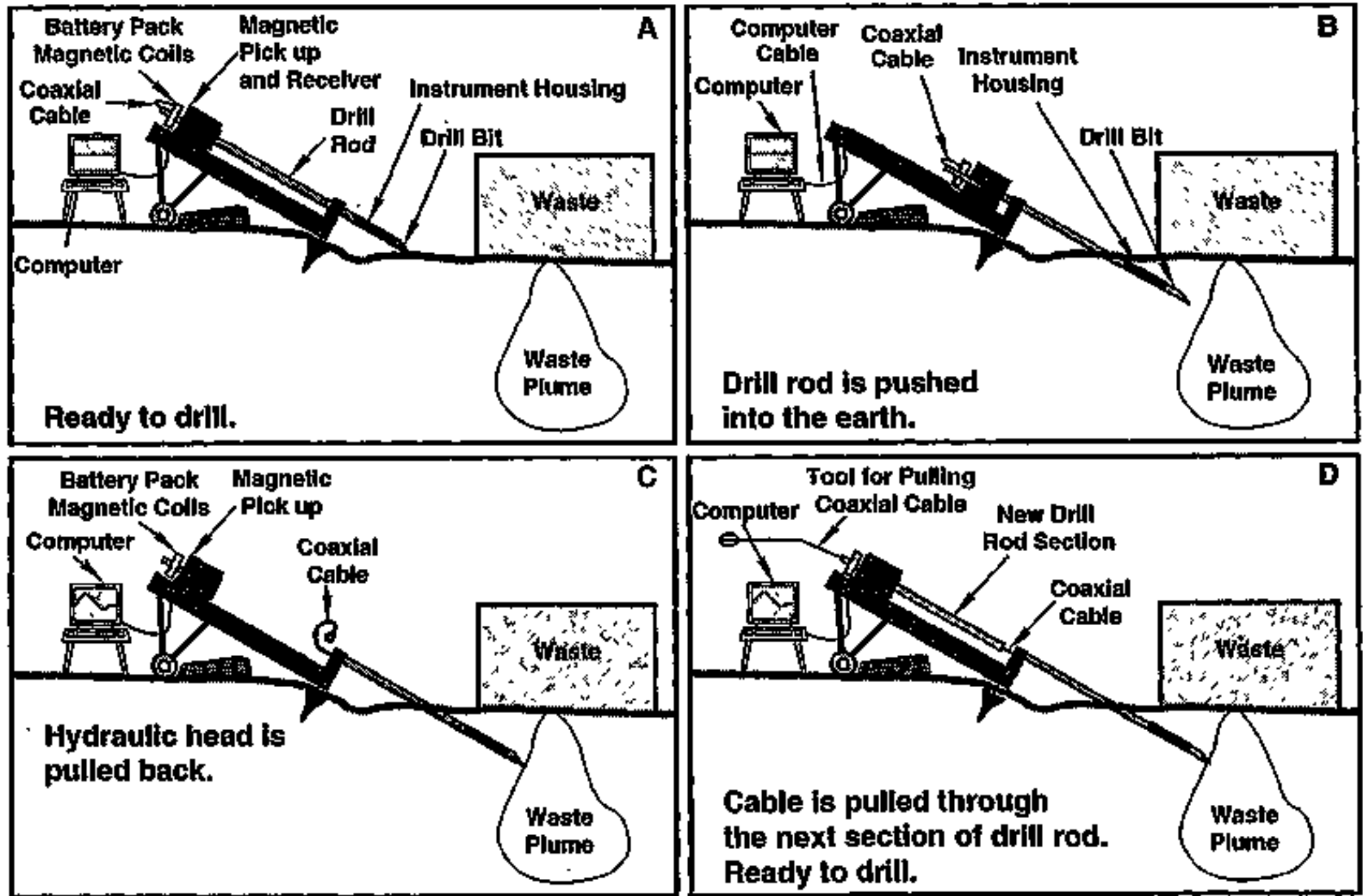

Figure 4. The Environmental Measurement-While-Drilling process ussing a coaxial spool. 
array. This small-surface mounted integrated circutt (IC) contains some 2000 logic gates. The Actel 10203 controls the data-stream format, logic clock, and circuit interfaces. The Actel $1020 \mathrm{~B}$ is programmed to provide the serial bit stream as bi-phase and non-return to zero (NRZ) digital. These two formats cover a wide range of communications systems including fiber optic, bardwire, and radio frequency (RF).

The system has a bit rate of 2400 bps. However, the bit rate can be increased easily. A practical limit to this FM system is $-30,000$ bps. However, if the signal coupling at the surface continues to be strong and noise-immune, the Actel bi-phase output could drive the coaxial system directly. The bi-phase date rate could exceed 100,000 bps. Data rates that high are approaching imaged data requirements.

Another important attribute of adaptability is to provide different supply voltages for different sensors. Only battery power (30V) is supplied on the coaxial cable. Once recejved, this voltage is converted to four different voltages: $+12 \mathrm{~V},-12 \mathrm{~V},+5 \mathrm{~V}$, and $+5 \mathrm{~V}$. A DC-to-DC converter generates these different voltages. The converter increases battery life by reducing current drain from the batteries and allowiag the battery voltage to range from $18 \mathrm{~V}$ to $32 \mathrm{~V}$ without affecting sensor electronics. A second DC-to-DC converter generates the $900 \mathrm{~V}$ GRS bias voltage. Current requirement for the down-hole electronics is only $32 \mathrm{ma} @ 30 \mathrm{~V}$.

\subsubsection{Drilling Platform}

The drilling platform for this demonstration is a J2511, a directional boring system using minimal drilling flujd and fluid mizer pipe. The boting system is supplied by Geneva Corporation-Ditch-Witch of Georgia This system uses a high-frequency electromagnetic beacon with a walk-over monitor to measure drill bit location and depth.

\subsubsection{Down-hole Components}

Down-bole components of the gamma ray detection sensor system being demonstrated consist of a gamma ray spectrometer, a multichannel analyzer, a $900 \mathrm{~V}$ power supply, a signal conditioning and transmitter board, and a coil containing coaxial cable for transraitting dafa to the surface. The down-hole components are contained within 0 -ring sealed aluminuma tubes to protect them from the drilling environment.

\subsubsection{Up-hole Components}

The up-hole system consists of a battery pack/coil, pickup coil, receiver, and a personal computer. During driling, the system monitors (1) gamme radiation by gamma ray spectrometry, (2) the $+i 2 \mathrm{~V}$ and $-12 \mathrm{~V}$ required at the down-hole signal conditioning and transmitter board, (3) the up-hole battery volage as measured down-hole, and (4) two temperatures associated with the detector and instrunentation.

Since human safety is a primary consideration, reliability and high data surety are prionity systen requirements. To meet this requirement, the system design incorporates dafa quality assurance techniques to ensure data reliability. The basic format used in the EMWDGRS systern is also used in the weapons complex for very high data surety where destructive 
testing may cost hundreds of millions of dollars and getting "one shot" reliable data is imperative.

\subsubsection{Cabje Deployment System}

The cable is contained in a spool located with the down-hole components of the system. The cable passes through the drill pipe and connects to the up-hole components. The cable is threaded through each added section of drill pipe and unspools as the drill pipe is advanced. The cable from the down-hole instrument package is pulled through each piece of drill pipe and through the drilt head to the battery pack/coil mounted on a spindle at the rear of the drill head. Because the cable connection must pass through the drili pipe, which is restricted to about $5 / 16^{\text {n }}$ (7.9 mm) at each pipe section pin, a $0.25^{\prime \prime}(6.35 \mathrm{~mm}$ ) outsido diameter (OD) Lemo coaxial connector is used. The cable is sealed as it passes through the spindle at the battery pack/coil. The spindle leads to the drill ffuid handling system. Drilt fluid pressure is normatty in the range of $300 \mathrm{psi}(1.435 \mathrm{kPa})$ to $500 \mathrm{psi}(2.392 \mathrm{kPa}$ ), but can go as high as $1500 \mathrm{psi}(7.177 \mathrm{kPa})$. A cord grip fitting is used to seal against the $0.07^{\prime \prime}(1.8 \mathrm{~mm}) \mathrm{OD}$ coaxial cable. The sealing grommet in the cord grip fitting is slit so that it can be removed from the cable, allowing the connector to pass through the body of the cond grip fitting. This arrangement has been tested to 600 psi (2.871 kPa) air, which is approximately $3000 \mathrm{psi}(14.354 \mathrm{kPa})$ water, without leakage. The coaxial cable is pulled through each section of drill pipe using a $16 \mathrm{ft}$ (4.88 m) long, 1/4* (6.35 $\mathrm{mm}$ ) diameter rod fashioned from two pieces of aluminum rod threaded together. A tip consisting of steel wire bent backward acts as a flexible guide as the rod is insented through the drill head and pipe from the spindle to the instrument housjng. This guide tip is replaced with a tip containing the mating Lemo connector. Thus, the cable is unspooled and is pulled through the drill pipe and head as the rod is withdrawn. The time required to add a new section of pipe, deploy the cable, and prepare to acquire data is an important parameter for evaluating the system's total performance.

\subsubsection{Data Collection System}

The data collection system is comprised of fonr parts: a computer, magnetic pick-up coil and receiver, battery pack and magnetic coil, and the down-hole electronics package. The coil couples the AC signal between the rotating drill pipe and the stationary coil and receiver, which are mounted on the drilling platform. The receiver converts the AC signtal into a serial bit stream. A computer equipped with a telemetry serial card receives the data and displays downhole measurements in real time.

The EMWD system provides real-time data on an 8 differential/single analog multiplexer and any number of digital channels. Sampling speed from analog channeis can reach $100 \mathrm{kHz}$. For the EMWD-GRS system, three digital chantels are used. Readings are taken at a rate of 20 per second. The telemetry system is programmable firmware that can easily support many different data fomats and adjitional data channels. The current format (Digital FM Biphase, 4800 baad) provides excellent noise rejection. A SNL-designed receiver removes FM carrier noise, generates data clock, and buffers data for use by an IBM or compatible persofial computer. A $28 \mathrm{~V}$ rechargeable battery pack can supply down-hole instrumentation power for more than 18 hours of drilling. A DC to DC converter increases battery life by reducing battery eurtent drain and allowing the battery voltage to range from 18 to $32 \mathrm{~V}$ without affecting sensor electronics and data quality. The battery pack remains topside for easy maintenance. 
This Page Intentionally Left Blank 


\subsection{DEMONSTRATION SITE DESCRIPTION}

\subsection{Sife Name and Location}

The selected demonstration site is the previously characterized SRS F-Area Retention Basin. It is located outside and south of the F-Area perimeter fence and east of burilding 281-8F and the Effluent Treatment Cooling Water Basin of the Savannah River Site near Aiken, South Carolina (Figure 5).

\subsection{Site History}

The F-Area Retention Basin was constructed as an unlined, temporary container for potentially contaninated cooting water from the chemical separations process and storm sower drainage from the F-Area Tank Farm. The bastin is approximately $200 \mathrm{ft}(61 \mathrm{~m})$ long, $120 \mathrm{ft}$ $(35.6 \mathrm{~m})$ wide, and 6.9 feet $(2 \mathrm{~m})$ deep and tas a total volume capacity of approximately 6,128 cubic yards $\left(4,685 \mathrm{~m}^{3}\right)$. When contamination was detected in the cooling water, the water was diverted to the retention basin or F-Area seepage basins. Contanination of this basin came from contaminated cooling water as well as from various spills or overflows at the basin (WSRC, 1995). The basin was in active use from 1955 until 1972, when it was replaced by a lined retention basin located to the west of the site. In 1978, the F-Area Retention Basin was excavated, backfilled with soill and covered with grass. A process sewwer line which extended from the basin to a diversion box located approximately $500 \mathrm{ft}$ north of the basin was abandoned. The site was evaluated through soil sampling in early 1979. The major radionuclides present incladed strontitun (\$r)-89/90 and cesium (Cs)-137. Sampling results indicated maximum Cs-137 concentrations of $1,410 \mathrm{pCi} / \mathrm{g}$ in the berm and $430 \mathrm{pCi} / \mathrm{g}$ in the basin floor. Maximum $\$ \mathrm{~S}-89 / 90$ concentrations in the basin berm were I,000 pCi/g (WSRC, 1995).

\subsection{Site Characteristics}

The F-Area Retention Basin was backfilled and covered with grass during 1978. Backfill in the basin extends to a depth of approximately $10 \mathrm{ft}(3 \mathrm{~m})$. The site is at an elevation of approximately $269 \mathrm{ft}(82 \mathrm{~m})$ in an area of tevel to slightly sloping topography. Surface dramage from the surnounding area flows toward Four Mile Branch, located approximately 3,937 $\mathrm{ft}(1200 \mathrm{~m})$ to the south (Figure 6). The slope toward Four Mile Branch is very gentle in the area of the basin, but becomes steeper neer Four Mile Branch (Scott of al., 1987).

\subsubsection{General Savannab River Site Geology}

As described by GeoTrans, Inc. (1992), the SRS is situated in the Upper Atlantic Coasta] Plain Province on what is known jocally as the Aiken Platean. The elevation of this platealu varies from 270 to $400 \mathrm{ft}$ ( 82 to $122 \mathrm{~m}$ ) above mean sea level (msi). Numerous streams have dissected the plateau into broad interstream uplands with narrow, steep-sided valleys. The geologic units range from Cretaceous to Recent in age and consist primarily of unconsolidated, interbedded gravets, sands, silts, and clays that were deposited upon a sloping crystalline basement andor Triassic bedrock. This wedge of sediments exhibits a gentle dip and a general increase in thickness to the southeast. Individual units display variable thickness and limited aerial extent. Figure 7 presents the stratigraphic units underlying the SRS. 


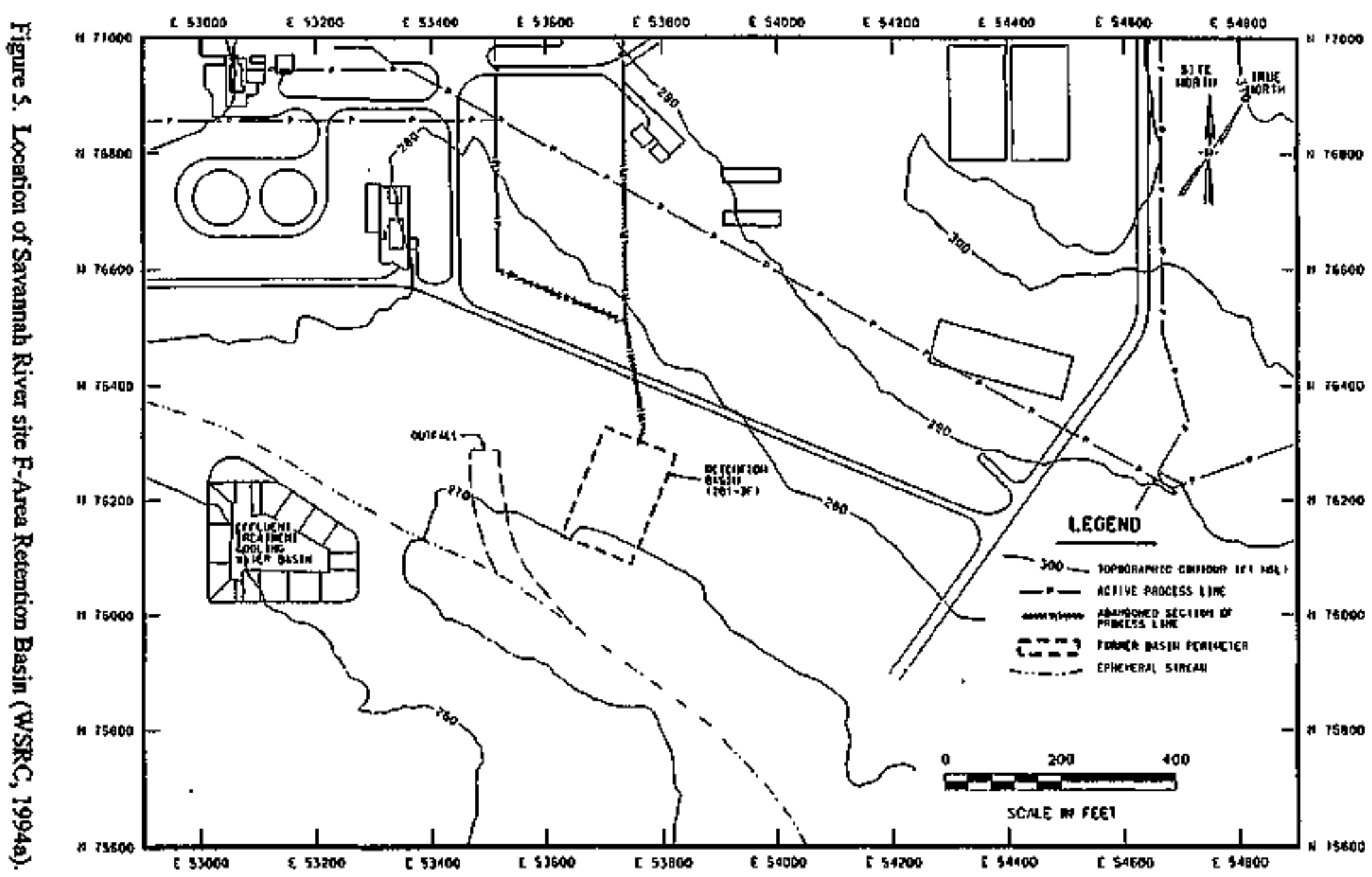




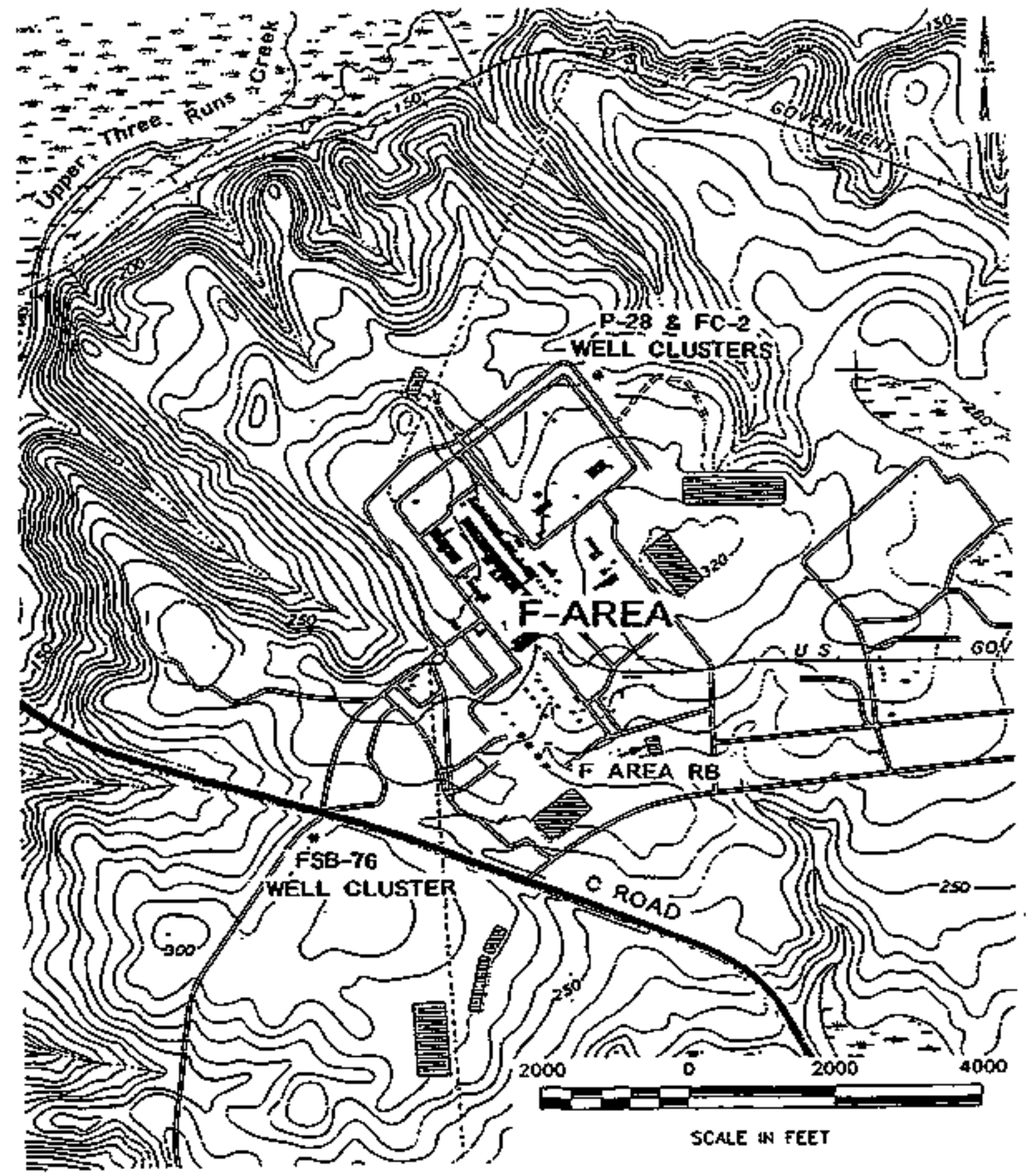

Figure 6. Topographic map of F-Area Retention Basit and surrounding area (WSRC, 1994a). 


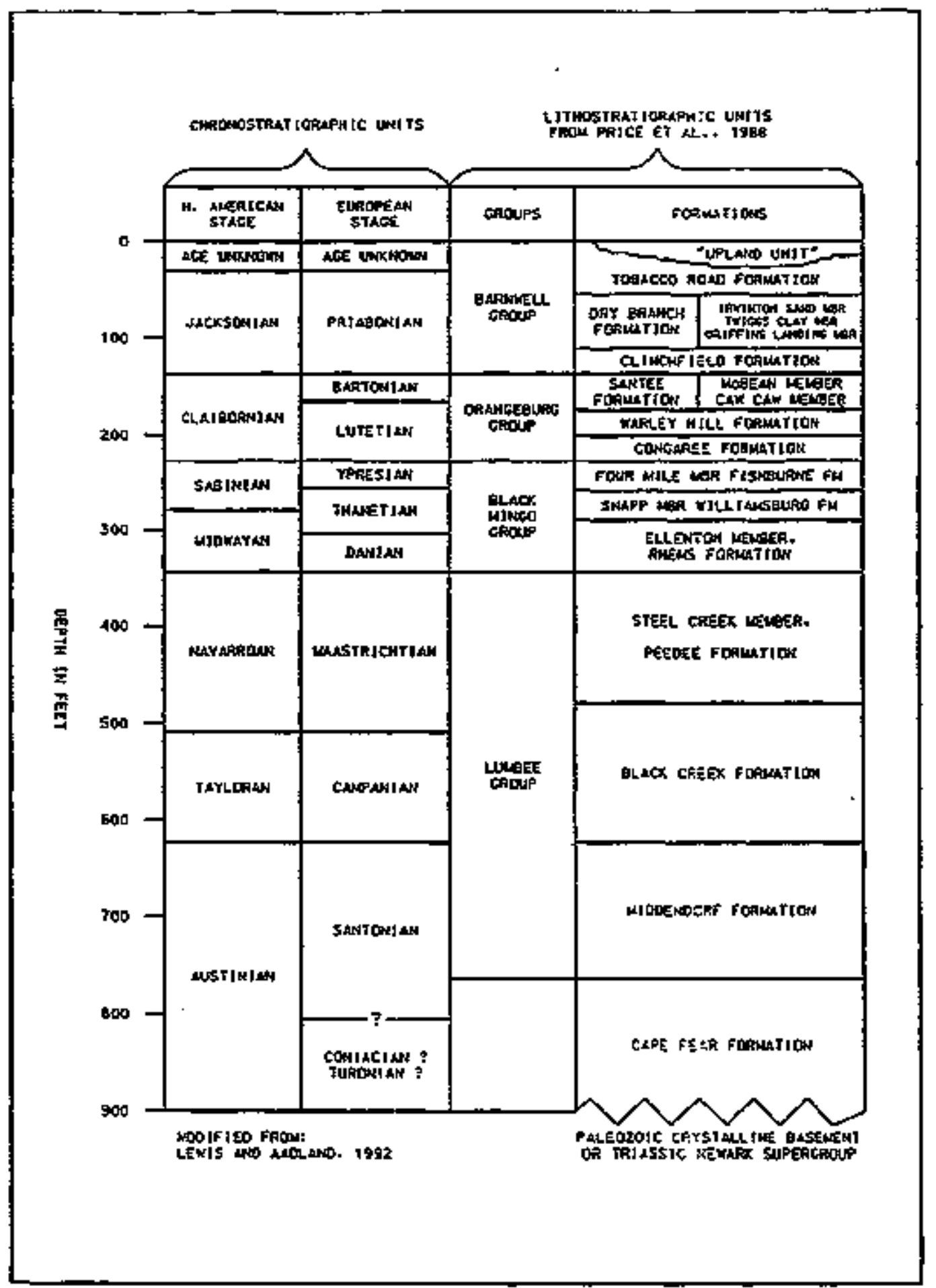

Figure 7. General stratigraphic units underlying the Savannah River Site (WSRC, 1994a). 
The near surface sediments at SRS are identified as the Tobacco Road Formation and the "upland unit." The Tobacco Road Formation consists of clays, silty clays, and massive sands. The "upland unit," a mapable strafigraphic unit which caps most of the upland areas at SRS, varies in lithology from coerse gravels to silty clays (WSRC, 1994a).

\subsubsection{F-Area Retention Basin Site Soils}

Geotechnical analysis of the soils at the F-Area Retention Basin was not available during the development of this demonstration plan. As discussed in Section 5.3.1, the near surface soils range from coarse gravels to silty sands. Solls in the vicinity of the F-Area Retention Basin are expected to range from clays to sands.

\subsubsection{Site Aydrogeology}

The hydrogeologic system at the SRS is comprised of both unconfined and confined aquifers. Figure 8 presents a comparison of the hydrostratigraphic units that have been used at SRS. Discussion of hydrogeology in this section is limited to the water table aquifer since the EMWD-GRS system will be denonstrated above the water table within in the vadose zone. Depth to groundwater at the site is approximately $70-85 \mathrm{ft}(21.3-25.9 \mathrm{~m})$ based on second quarter 1990 groundwater data; however, studies conducted over the years indicate that the water table could be as shallow as $52 \mathrm{ft}$ (15.8 m), depending on precipitation (WSRC, 1994a).

There are only three water table monitoring wells (FM series) at the F-Area Retention Basin, and all are positioned down gradient and to the southeast, soutiwest, and west of the FArea Retention Basin (Figure 9). These wells are part of a series of ten water table wells installed in late 1961 using aluminum casing and reportedly are no longer monitored (WSRC, 1994a). The nearest routinely monitored water table wells down gradient to the basin are the FET series wells. The FET series wells surround the effluent treatment cooling water basin located approximately $400 \mathrm{ft}$ (122 m) southwest.

Groundwater data collected is 1993 through the SRS grolndwater monitoring program indicated that the water table aquifer underlying the basin flows to the southwest (Figure 10). The hydraulic gradient of the water table is approximately $0.01 \mathrm{ft} / \mathrm{ft}(0.003 \mathrm{~m} / \mathrm{m})$ to the southwest based on 1993 first quarter groundwater data and as calculated from Figure 10 (WSRC, 1994a).

\subsubsection{Site Contaminants and Distribution}

All soil samples collected during the Phase I and IA investigations were analyzed for:

- Inorganics/metals

- Organics

- Radionuclide indicators and radionnclides

Analytical results for samples collected to determine background levels are found in Appendix A. 


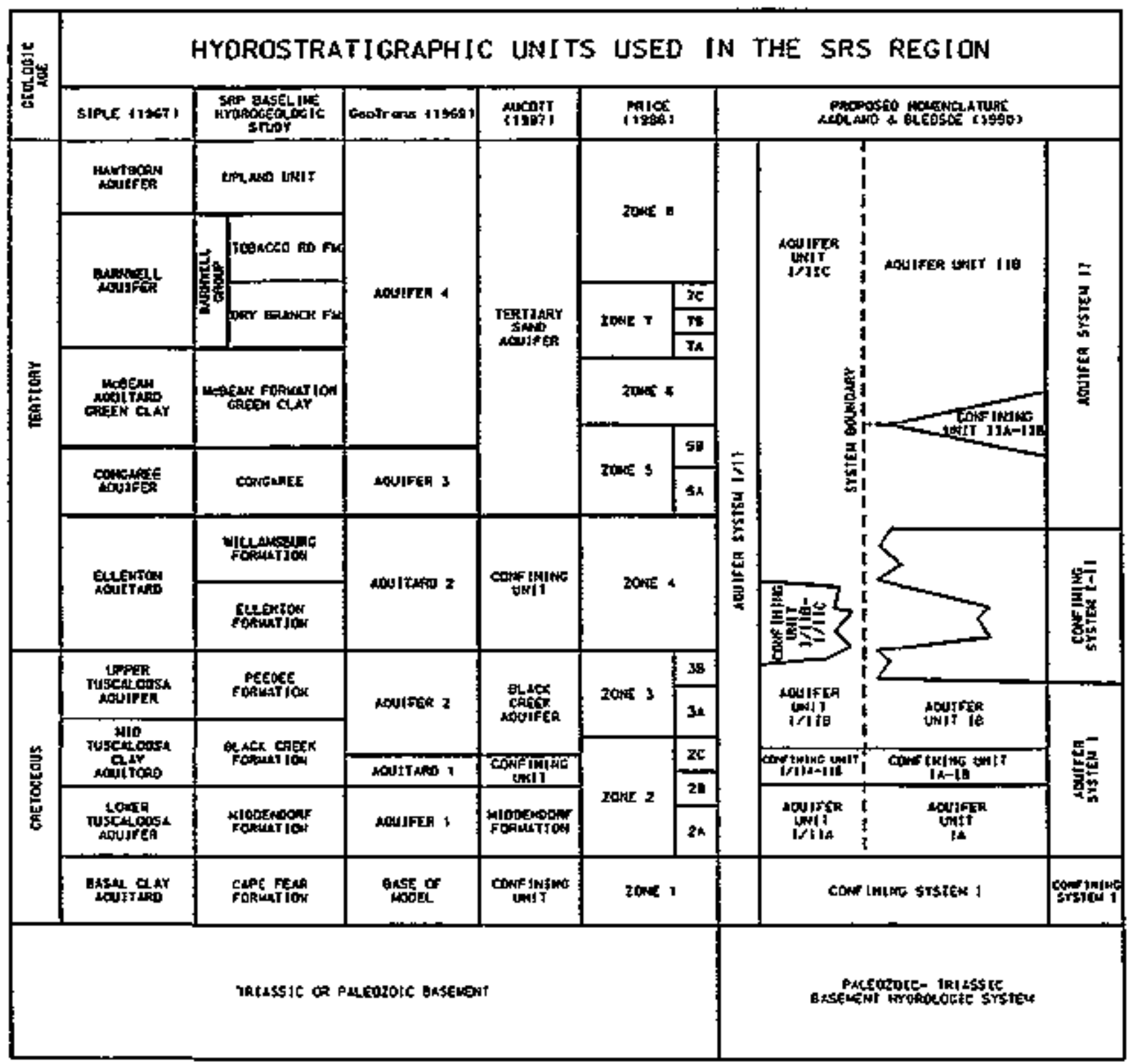

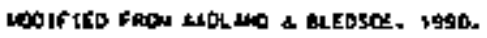

Figure 8. Hydrostratigraphic units underlying the Savannah River Site (WSRC, 1994a). 


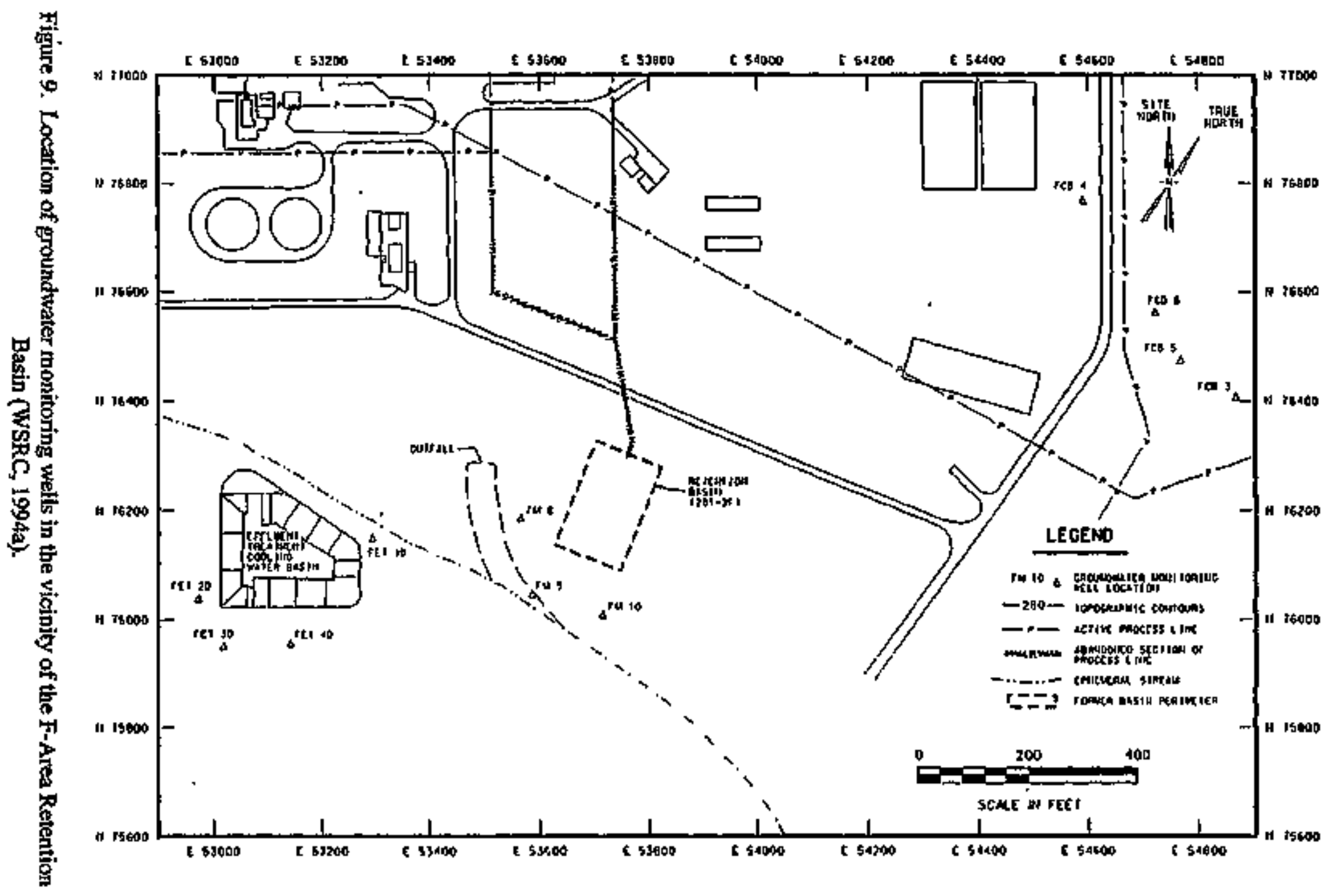




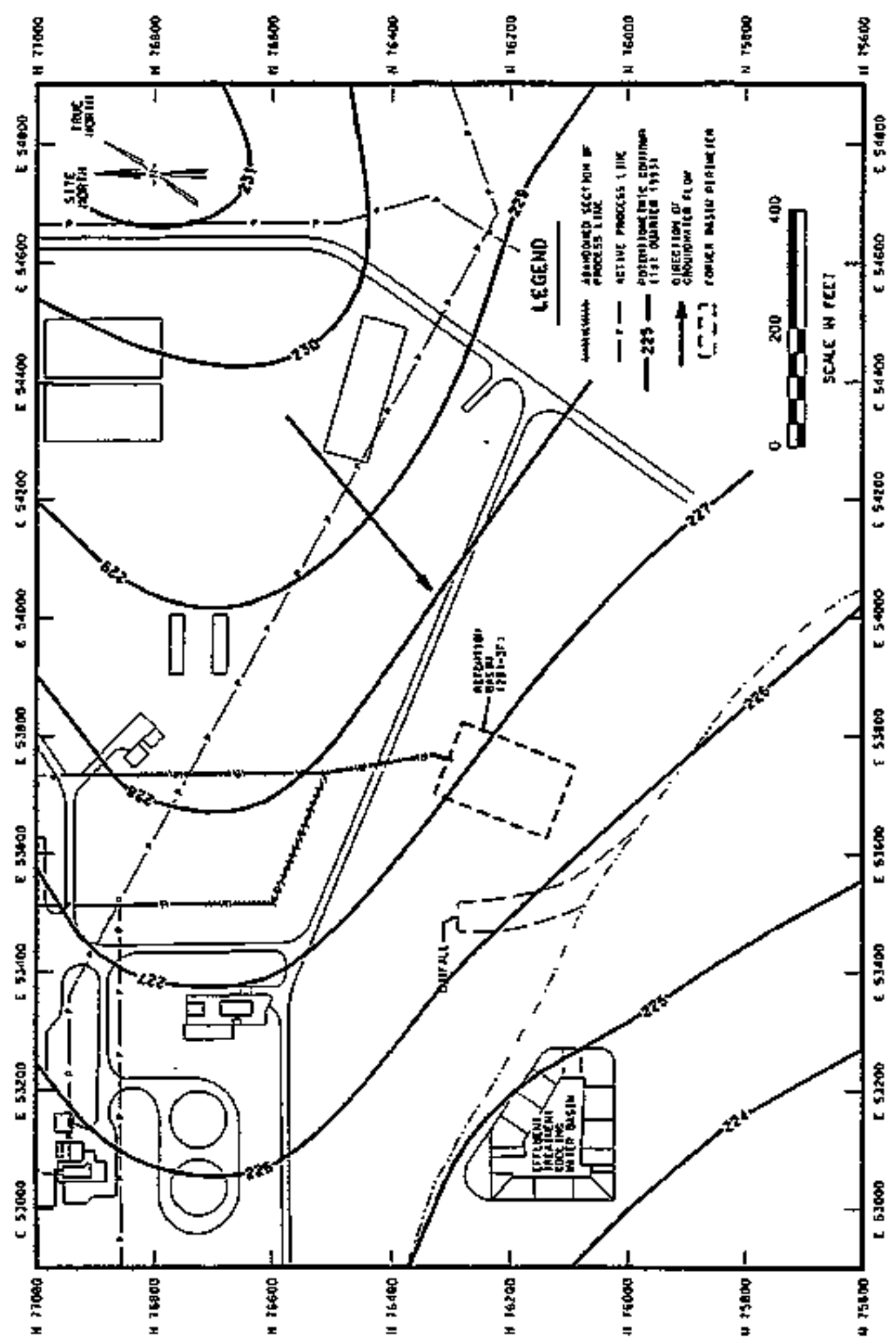

Source: adapted from GSA Water Table Surface Open File Map, 1Q93 Dara

Figure 10. Potentionetric surface of the water table (Aquifer Unit IIB) at the F-Area Retention Basin (281+35) (WSRC, 1994a). 
Results of the Phase I and Phase IA investigations were nsed to identify contaminants of concern (COC) in the F-Area Retention Basin (WSRC, 1994a). The identified COCs for the FArea Retention Basin are listed in Table 2.

Table 2. Contaminants of Concern at the SRS F-Area Retention Basin (WSRC, 1994a)

\begin{tabular}{|c|c|c|}
\hline Badionuclides & Organics & Mefals \\
\hline Tc-99 & Carbon Disulfide & Barium \\
Cesium-137 & Di-r-Butyl Phthalate & Berylium \\
Strontium-90 & N-ritrosodiphenylamine & Lead \\
Carbon-14 & Di-benzofuran & Nickel \\
Americium-241 & Toluene & Thallium \\
Cobalt-60 & Methylene chloride & Arsenic \\
Barium-133 & TCE & Chromium \\
Europium-154 & 2 Chloro phenol & \\
Potassuim-40 & & \\
Uranium-238 & & \\
Nickel -63 & & \\
Plutonium-239/240 & & \\
Radium-226 & & \\
Iodiue-129 & & \\
\hline
\end{tabular}

Radionuclide continninant distributions for Cs-137 and Sr-90 at samplo locations FRB$05,06,07,08$, and 19 are shown in Ftgures 11 and 12 . As illustrated, the greatest concentrations of Cs-137 occur between the depths of 9 and 19 feet (Figure 11). The greatest concentrations of Sr-90 fall within the depth range of 9 to 28 feet (Figure 13). Analytical results for sample locations FRB-05, 06, 07, 08, and 19 are listed in Appendix B. 


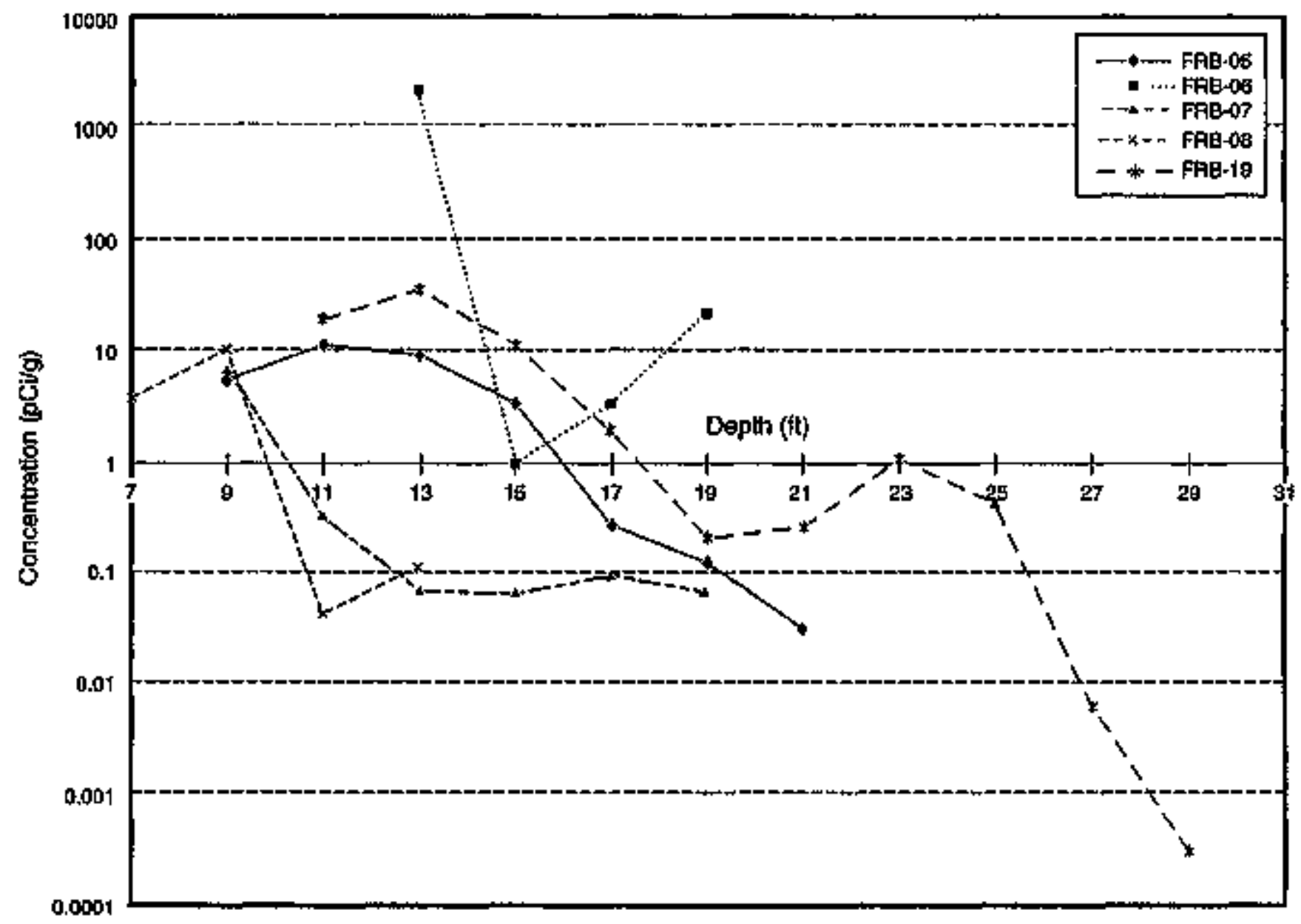

Tentegr-14a 0

Figure 11. Concentration $(\mathrm{pCi} / \mathrm{g})$ of $\mathrm{Cs}-137$ rolative to depth on sampling locations FRE-05, 06, 07, 08, and 19 within the S1kS F-Area Retention Basin. 


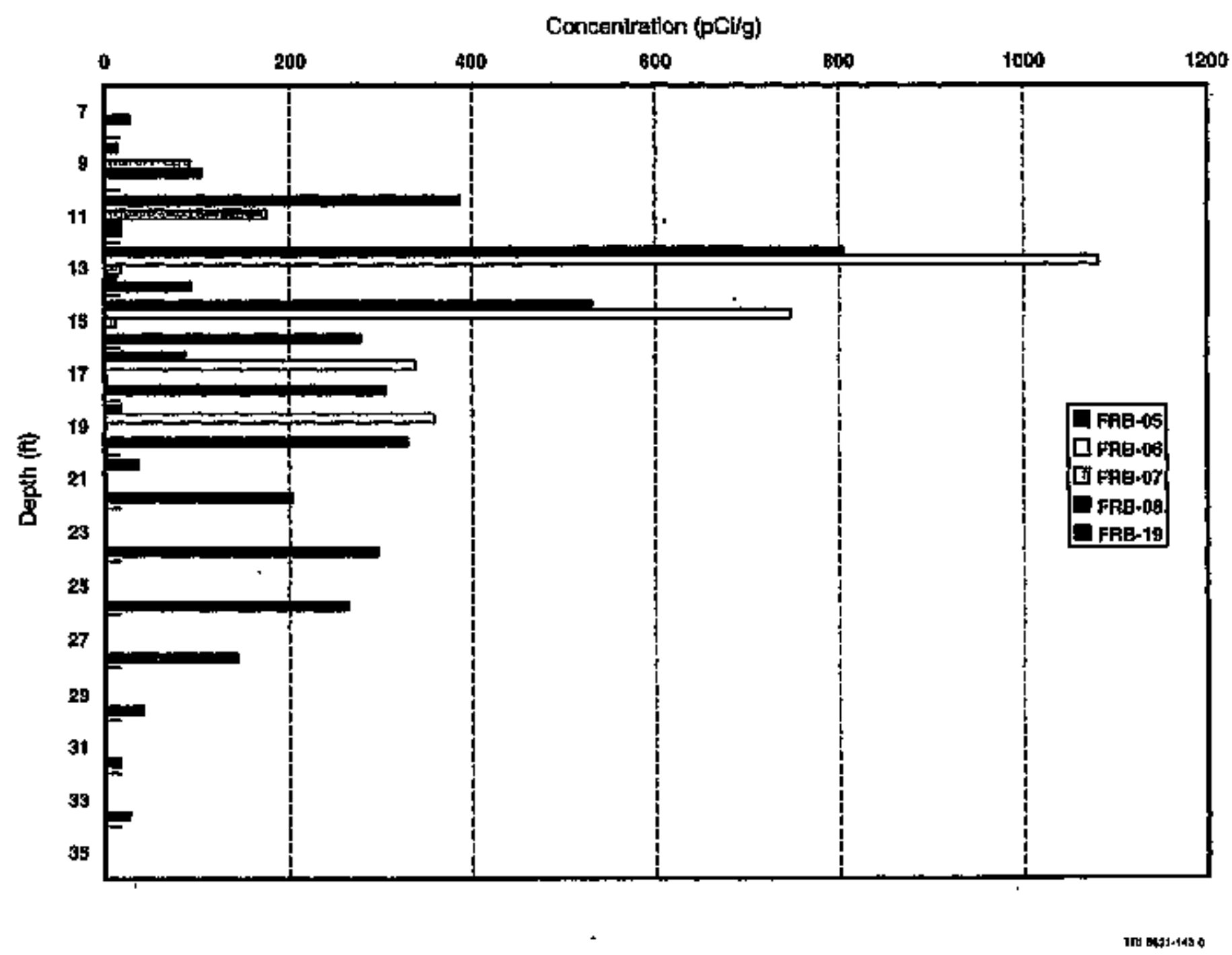

Figure 12. Concentration (pCi/g) of \$r-90 rejative to depth at sampling locations FRB-05, 06, 07, 08, and 19 within the SRS F-Area Retention Basin (user defined from WSRC, 1995). 
This Page Intentionally Left Blank 


\subsection{DRILLING AND DATA COLLECTION PLAN}

\subsection{Overview of Drilling and Evaluation Operations}

Phase I of the demonstration at the F-Area Retention Basin will determine Cs-137 background conditions. This will be accomplished by dritling one hole approximately $50 \mathrm{ft}$ (15.3 $\mathrm{m}$ ) in length at a depth ranging from 10 to $15 \mathrm{ft}$ ( 3 to $4.5 \mathrm{~m}$ ) at a radiological "clean" area adjacent to the F-Area Retention Basin. Investigation derived waste generated from this background determination will be considered non-coneminated and disposed of at the borehole location. After background conditions are determined, the EMWD-GRS system will be demonstrated in the previously characterized F-Area Retention Basin.

Phase II of the demonstration at the F-Area Retention Basin witl consist of monitoring environmental conditions in real time while drilling two boreholes daylight-to daylight. These holes will pass near four sample locations, FRB-05 through 08, and adjacent to sample location FRB-19. Values of contaminant levels are kmown at all five sample tocations. The contaminant levels continuously recorded by the EMWD-GRS system during ditling will be compared to contaminant levels previously determined through quantitative laboratory analysis of soil samples collected at locations FRB-05, 06, 07, 08, and 19 (WSRC, 1995). Results of the demonstration will be presented in report format.

\subsection{Communications, Documentafion, Logistics, and Equipment}

The SNL project leader for the demonstration will communicate regularly with the demonstration participants to coordinate all field activities associated with this demonstration and to resolve any logistical, technical, or quality assurance (QA) issues that may arise as the demonstration progresses. The successful implementation of the denonstration requires detailed coordination and constant communication between all demonstration participants.

All demonstration field activities will be thoroughly documented. Field documentation will include field logbooks, photographs, field data sheets, and electronic data files.

The SNL freld team leader will be responsible for maintaining all field docuntentation. Field notes will be kept in a bourt logbook. Each page will be sequentially numbered and labeled with the project naurte and number. Completed gages witl be stgned and dated by the individual responsible for the entries. Eirors wit] baye one line drawn through them, and this line will be initialed and dated.

All photographs will bo logged in the field logbook. These entries will inclade the time, date, direction, subject of the photograph, and the identity of the photographer. Specific notes about data collected will be written in the field logbook. Any deviations from the approved final demonstration plan will be thoroughiy documented in the fietd logbook by the SNL project leader.

The SNL demonstration tearn will obtain the EMWD-GRS equipment and contract drilling services for this demonstration. The drilling confractor will provide for decontamination of equipment and personnel as required by 29 CFR 1910.120-Hazardous Waste Operations and 
Emergency Response. Westinghouse Savannah River Company witi provide

equipmentmaterials necessary for disposal of any radioactive/hazardous waste gemerated.

\subsection{Data Collection Procedures}

The data collection procedures focus on identifying the drilling locations, reading locations, and decontamination and disposal of any generated waste as discussed in the following subsections.

\subsubsection{Drilling Locations}

The drilling locations for the two daylight-to-daylight boreholes are shown in Figtre 13. The boreholes will be drilled horizontally at a depth of approximately $15 \mathrm{ft}(4.5 \mathrm{~m})$, which is $5 \mathrm{ft}$ $(1.5 \mathrm{~m})$ below the clean backfill in the retention bassin. The drill rig will be positioned east of the backfilled retention basin at a location to be determined by drilling protocol. Forizontal Borehole No. I will be drilled near previonsly drilled and sampled vertical boreholes FRB-05, FRB-06, and FRB-19. Horizontal Borehole No. 2 will be drilled near proviously drilled and sampled vertical boreholes FRB-07 and FRB-08.

\subsubsection{EMWD-GRS Data Collection}

The data collection procedures used with the EMWD-GRS system are the following:

1. Run decom software using default setup.

2. Start data storage by providing a file name.

3. With systen mounted behind the drill bit, test rotate the diilling rig while montoring wired connections and computer.

4. Stop rotating and place small check source (WSRC external cesjum sources) near the drill bit

5. Take reading for approximately 2 minutes.

6. After visual verification that the system is working, start the normal drilling process.

7. After completion of the drilling process, check the system once more with the freld check source.

8. Close the PC file and store file to secondary backup system.

The entire procedure for data collection is located in Appendix C.

\subsubsection{Decontamination and Disposal of Generated Waste}

The F-Area Retention Basin is subject to Comprehensive Emvironmental Response, Compensation, and Liability Act (CERCLA) requirements. The drilling contractor will provide for decontamination of equipment and personnel by 29 CFR 1910.120-Hazardous Waste Operations and Energency Response. The drilling operations will minimize waste by using fluid mizer pipe during drilling operations. The drill bit entry pit, approximately $3 \mathrm{ft}^{2}\left(\approx 1 \mathrm{~m}^{2}\right)$, will be lined with plastic and contain the minimal amount of drilling generated waste for appropriate disposal. Investigation derived waste generated during the technology demonstration will bo handled in accordance with site procedures foumd 


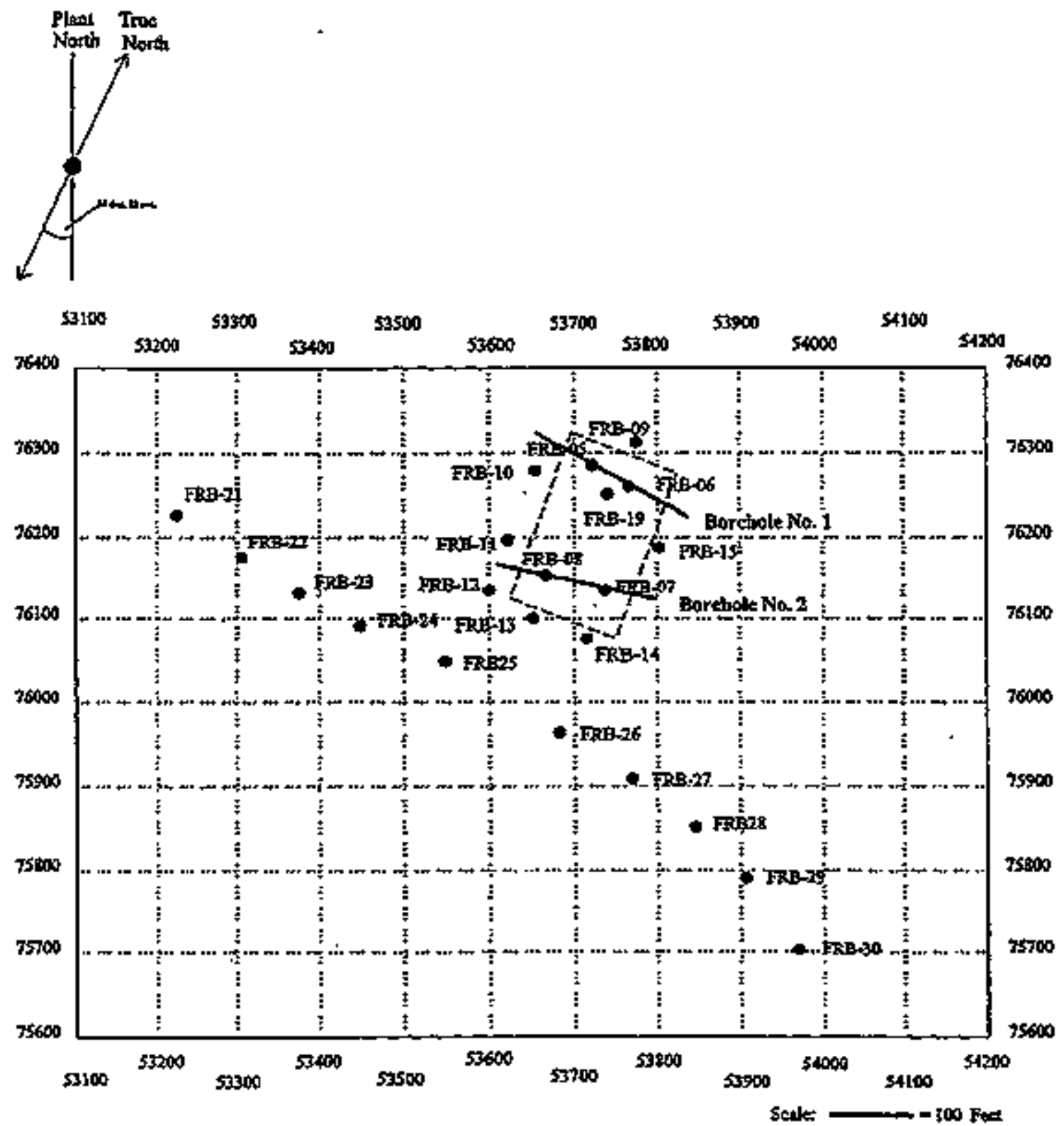

Figure 13. Losations for drilling continuously monitoned daylight-to-daylight boreholes at the Savannah River Site F-Area Retention Basin (modified from WSRC, 1995). 
in WSRC I S Manual (Procedure 3.10 and 3.15), Investigation-Derived Waste (IDW) Management Plan.

Radioactive, hazardous, and non-hazardous waste generated during the demonstration will be managed in accordance with SRS procedures and applicable regujations. Rags and material contaninated with gasoline, hydraulic fluid, diesel, etc., will be segregated, double bagged in plastic bags, and placed in controlled areas.

Radioactive and hazardous waste will be managed on site and transported for final disposition by Westinghouse Savannah River Company personnel. Westinghouse Savannah River Company is responsible for final disposition of IDW.

\subsubsection{Scheduie}

The demonstration is scheduled to take place April 22-26, 1996.

\section{Day 1}

During the morning secure badges and attend site specific training. In the afternoon, mobilize equipment at the "cold" test site adjacent to the F-Area Retention Basin and prepare for drilling horizontal borehole to determine background conditions

Dav 2

Drill borehole at the "cold" test site to determine background conditions. Relocate equipment to the F-Area Retention Basin and set up for dirilling horizontal Borehole No. 1. Borehole No. 1 will pass near previously sampled locations FRB-05, 06, and 19.

\section{Dav 3}

Drill horizontal Borehole No. 1. Upoin completing Borehole No. 1, move equipment to the horizontal Borehole No. 2 location. Borehole No. 2 will pass near previousty sampled locations FRB-07, and 08.

Day 4

Drilt horizontal Borehole No. 2 .

Day 5

Decontaminate equipment, Demosilize, and move off location. 


\subsection{QUALITY ASSURANCE PROJECT PLAN}

The quality assurance project plan (QAPP) for this demonstration specifies procedures that will be used to ensure data quality and integrity. Careful adherence to these procedures will ensure that data generated by the demonstration will meet the desired performance objectives and will provide sound analytical results.

\subsection{Purpose and Scope}

The primary purpose of this section is to outline steps that will be taken by operators of the EMWD-GRS system to ensure that data resulting from this demonstration is of known quatity and that a sufficient number of critical measurenents are taken.

\subsection{Quality Assurance Responsibilities}

The SNL project leader is responsible for coordinating the preparation of the QAPP for this demonstration. The SNL project leader will ensure that the QAPP is implemented during all demonstration activities. The SNL QA manager for the demonstration will review and approve the QAPP and will provide independent QA oversight of al! demonstration activities.

Readings will be taken and analyzed on site using the EMWD.GRS system technology. These readings will be compared to previously collected samples analyzed using U.S.

Environmental Protection Agency (EPA) approved methods. The SNL project leader, field team leador and technology operator wilt be responsible for data readings and analysis quality assurance/quality control $(\mathrm{QA} / \mathrm{QC})$ throughout the demonstration. Primary responsibility for enserring that the data acquisition activities comply with the requirenents of the data acquisition plan will rest with the SNL field team leader. QA/QC activities for the EMWD-GRS system will include activities to assure that the demonstration will provide data of the necessery quality.

\subsection{Data Quallity Parameters}

The data obtained during the demonstration must be of sonnd quality for conclusions to be drawn on the performance of the EMWD-GRS system. For all measurement and monitoring activities conducted for the EPA, the Agency requires that data quality parameters be established based on the proposed and uses of the data. Data quality parameters include six indicators of data quality: representativeness, completeness, comparability, accuracy, precision, and lowerlimiting the detection threshoid for elimination of false-positive readings.

Data generated by EMWD.GRS system technology will be compared to data generated from previous site characterization (Appendix B).

\subsubsection{Representativeness}

Representativeness refers to the degree to which the data accurately and precisely represent the conditions or characteristics of the given parameter. In this demonstration, representativeness will be ensured by executing consistent data coltection procectures, including reading locations, reading proceduses, and data storage as previously outlined in Section 6.3.2. 
Representativeness also will be ensured by using each method at its optimum capability to provide results that represent the most accurate and precise measurement each method is capable of achieving.

\subsection{Completeness}

Completeness refess to the amount of data collected from a measurement process compared to the amount that was expected to be obtained. For this dernonstration, completeness refors to the proportion of valid, acceptable data generated using each method.

The completeness objective for data collection during this demonstration is to take spectral readings continuously during the drilling process. The drilling process will continue under a waste site for approximately $150 \mathrm{ft}(45.7 \mathrm{~m})$. Never before has such a complete data set been taken. Readings at selected points corresponding to soil sample locations will be compared.

There is a physical Iimitation to the sensitivity and resuiting range of spectral gamma measurement. These limitations relate to crystal size and maximum data collection rates. Expected range of 3 to $1000 \mathrm{pCi} / \mathrm{g}$ is the ideal system imitation.

\subsubsection{Comparability}

Comparability refers to the confidence with which one data set can be comapared to another. The primary objective of this demonstration is to evaluate how well the EMWD-GRS systom technology performs in determining the presence or absence of Cs-137 compared to analytical data derived from previons sito assessment.

Analytical methods have the unique distinction of setfing the questionable EPA standard. In situ measurments as determined by the EMWD-GRS system have limiting accuracy and range of waste analyst (t.e., the gamma radiation range is expected to vary from material background to the amount of C $\$ 137$ remaining after approximately 7 half lives). If "high" levels of gamma contamination exists at higher energies, the range may be wider than expected. Other more comparable systems can detect radiation levels within some reasonable range from natural occurring sources as uranium, thorium, and potassium. These systerns have been adapted for environmental applications to add Cs-137 and cobalt (Co)-60 to the list. The process of unfolding energy peak and count rate information into gamma flux density, relating to waste concentrations within soils, has not been fally realized. Calibration procedures consistent with industry's best practices are being used while looking at new more innovative approaches. The expectation is to be consistent with off-site anatysis methods.

\subsection{Aceuracy}

Accuracy refers to the difference between the contamination readings and a reference standard. The resulting accuracy contains error variances from both the repeated contanination readings and the reference standard. The analytical reading from sampled soil will be the standard. The analytical reading is a single valued number with error bars. The EMWD-GRS system readings are a function of contamination reading versus location and sample period. ideally, the EMWD-GRS system would be exposed to soils with uniformly distributed 
contamination consistent with the small sample used in laboratory ansilytical measurement. These ideal conditions will not exist during the demonstration.

Using the statistical nature of the gamma process and calibration information taken from controlled calibration pits at Grants, New Mexico (Leino et. al, 1994), the coniparable arciracy will be established. Second calibration standards for Cs-137 will be established using previously sampled locations at a DOE facility. Calibration procedures are listed in Appendix D.

Historically, it has been understood that the standard deviation of the gamma count rate is equal to the square root of the measured count. The measured count increases has the sample period increases. For example, a deviation of $10 \%$ must have a measured count equal to 100 . To jucrease accuracy, controls must be piaced on the rate of drilling. The limiting drilling rate has not been established. The industry drijling standard for natural gamma backgrounds is 2 to $10 \mathrm{ft}$ per minute.

\subsubsection{Precisjon}

Precision refers to the degree of mutual agreement among individual measurements and provides an estimate of random error. Precision will be established using WSRC external cesiutin sources for calibration and checks of the EMWD-GRS tool for response of Cs-137 in native soil. The EMWD-GRS system collects data at a sufficiently high rate so that the driliing rate does not affect data quality in moderately to highly contaminated soils. If a radiological "hot spot" is encountered, the drilling rate can be reduced to provide sufficient radiation cournt statistics. As discussed in the concluding paragraph of Section 7.3.4, randorn eroor is directly related to sample period, dritling rate (changing enviromments), and contamination levels (count rate). For greater precision at low contamination levels, the sample period must be increased and drilling rate reduced, if not stopped altogether.

\subsubsection{Probability of False-Positive}

The probability of a false-positive is the probability of a contaminated soil condition that does not exist.

The statistical nature of gamma measurements and electronic noise in any garnma measurement system does not provide a true zero reading. Given any sample period, there is a probability of measuring gajnma relating to the energy levels of waste material as Cs-137 even witbout any laboratory-measurable quantities present. This problem requires setting the

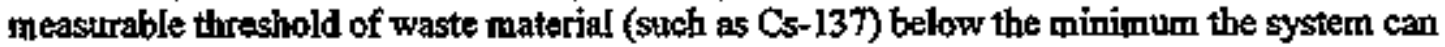
meastro accurately. This lower limit is an important parameter in proventing unwanted expense. The lower limit is a function of the sample period and frilling rate. The lower limit will be determined through background measurements at the radiologitally "cold" site adjacent to the FArea Retention Basin at the SRS. Approximately 100 measurements will be taken to statistically parameterize the backsround radiation count rates. The lower limit will be consistent with other off-site analysis methods. 


\subsection{Callbrafion Procedures, Quality Control Checks, and Corrective Action}

This section describes the calibration procedures that apply to the EMWD-GRS technology. It also contains a discussion of the corrective action to be taken if the $Q C$ parameters fall outside of the cvaluation criteria.

\subsubsection{Calibration Procedures}

Calibration will be consistent with procedures in Westinghouse Hanford WHC-SD-ENTI-293, Rev. 0 and with Grand Junction calibration techniques for the Grants, New Mexico, calibration pits. The Westinghouse Hanford document covers the procedures for calibrating gamma-ray wel! logiting tools using Hanford formation models. Calibration procedures for the GRS-EMWD system are located in Appendix $\mathbf{D}$.

In general, a complete calibration is required every six months with an initial check before and after each drilling operation. Complete calibration every six months can be performed at calibration pits at any DOE calibration facility. The calibration to be performed has two stages. First, the linear energy peak used to channel number calibration normally uses several radioactive elements of differing gamma energy emissions. Second, the ganma flux correlation to waste concentrations requires test pits of known flux and contamination levels. These pits can be found at a number of DOE facilities, but those best-known and used most frequently are the $A$ and $B$ models located at Grand Junction, Colorado.

Complete calibration generates a set of coefficients correlating spectral counts rates at differing energy locations to amounts of soil contamination. Should these coefficients exceed limits or fail to produce acceptable results, the system must be recalibrated or repaiked at Saadia National Laboratories, Albuquerque, New Mexico.

Initial check before and after drilling uses a simple potassium-40 $(\mathrm{K}-40)$ (or other common isotope) of known energy emission. This then becomes part of the data file for validation of system operation while the driling measurements are being taken. A simple visual inspection of the initial check data is sufficient to approve the start of diilling. A failed spectrum would reguire replacement of failed system components. This work could be done in the field.

\section{4 .2 Soil Sample Analysis}

The data to be used for evaluating the EMWD-GRS system were collected during the site assessment of the F-Area Retention Basin. Sample collection and analysis methodologies, including quality control procedares, have been fully described in WSRC, 1995.

\subsection{Data Reduction, Validation, and Reporting}

To maintain good data quality, specific procedures will be followed during data reduction, validation, and reporting. These procedures are detailed bejow. 


\subsubsection{Data Redaction}

During the data reduction process, data will be reduced to the following formats:

- Total count rate as a function of time.

- Cs-137 count rate as a function of time.

- Spectra taken near previously sampled locations.

\subsubsection{Data Validation}

The SNL field team leader will verify the completeness of the appropriate data forms and the completeness and correctness of data acquisition and reduction. The field team leader will also review calculations to verify accuracy, completeness, and adherence to the specific technology protocols. Calibration and QC data will be examined by the SNL field tean leader, SNL ga manager, and SNL project leader. Project leaders and QA managers will verify that all instrument systerns are operational and that $Q A$ objectives for accuracy, completeness, and method detection limits have been met.

\subsubsection{Data Reporting}

The Final report will utilize the WSRC 1995 (ESH-EMS-950563) analysis type format for presenting a comparison between BMWD-GRS generated data and previous site characterization laboratory analysis. Interpretation of the EMWD-GRS data will be presented in an effort to draw corollaries to empirical data if direct comparison of dafa is not possible.

\subsection{Data Quallity Indicators}

Data transnission quality and spectral gamma quality are the two data quality indicators used with the EMWD-GRS system.

Data transmission quality indicators are built into the system. When data is collected it is sent to the computer three (3) times. The counts from each data transmission are totaled and compared to verify they are the same.

Spectral gamma quality indicators are determined by verifying the functionality and approximate calibration of the GRS asing laboratory calibration sources. The functionality of the GRS is also verified while drilling by monitoring background peaks.

\subsection{Performance and System Audits}

The following audits will be performed during this demonstration. These audits will determine if this demonstration ptan is being implemented as intended. 


\subsubsection{Performance Audit}

The EMWD-GRS system performance will be compared with samples collected and analyzed during previous site assessments.

\subsubsection{On-Site System Audits}

On-site system audits for field operations will be conducted upon request. These audits will be performed by the SNL project leader. After the andits, audit reports will be prepared and incorporated into the demonstration records.

\subsection{Quality Assurance Reports to Project Manager}

QA reports provide the necessary information to monitor data quality effectively. It is anticipated that the following types of QA reports will be prepared as part of this demonstration on forms developed for this demonstration.

\subsubsection{Stahus Reports}

The SNL field teant leader will prepare periodic reports for the project leader. These reports should discuss project progress, problems and associated corrective actions, and future scheduled activities associated with the demonstration. When probiems occur, the field team leader will discuss them with the SNL project leader, estimate the type and degree of impact, and describe the corrective actions taken to mitigate the impact and to prevent a recurrence of the probleans.

\subsubsection{Audit Reports}

Any $\mathrm{QA}$ audits or inspections that take place in the field while the demonstration is being conducted will be recorded for future use by the technology developer.

\subsection{Corrective Action}

Whenever a performance svaluation or a technical system audit indicates that the equipment is out of calibration, the system will be evaluated to determine the source of the problem. Results of the system evaluation will be recorded in the demonstration record along with the comective action taken. The SNL project leader will review, approve, and document all corrective actions. 


\subsection{DATA MANAGEMENT AND ANALYSIS}

SNL will masage and analyze the data prior to presentation of the report to Westinghouse Savannah River Company.

The SNL project leader is responsibje for obtaining reducing interpreting, validating, and reporting the data associated with the technology's performance. These data will be reported in hard copy and ejectronic format (i.e., spreadsheet). A data dictionary will be included as a text file on the same diskette containing the data.

Other items that must be included in the demonstration record are:

- field records;

- photographs, slides, and videotapes (copies);

- sample analysis from previous site assessment;

- electronic data files.

\subsection{EMWD-GRS Reading and Location Identification}

The Reading focations will be located geographically and recorded as part of the EMWD-GRS system data collection capability. Horizontal and vertical conrdinates are to be provided.

\subsection{Data Analysis and Comparison with Prevlous Site-Characterization Data}

The data collected during this demonstration will be presented in a documented format (WSRC, 1995) that allows comparison with previously collected sample data. Interpretation of EMWD.GRS data will be presented in an effort to draw corollaries to empirical data (WSRC, 1995) if direct comparison of data is impossible. 
This Paģ Intentionally Left Blank 


\subsection{HEALTH AND SAFETY PLAN}

\subsection{Site Speciffc Health and Safety Plan (HASP)}

The site specific HASP for the EMWD-GRS system demonstration of the SRS F-Area Retention Basin is listed in Appendix E.

\subsection{Health and Safety Plan Enforcement}

The SNL project leacter, field site supervisor, and site health and safety officer will be responsible for implementing and enforcing the health and safety provisions of the site-specific HASP. The SNL project leader bears ultimate responsibility for ensuring that all demonstration participants abide by the requirements of the HASP. The field site supervisor will oversee and direct field activities and is responsible for ensuring compliance with the site-specific HASP. The health and safety officer (HSO) has the ultinate responsibility during activities at the demonstration site. 
This Page Intentionally Left Blank 


\subsection{DELIVERABLES}

The deliverabie of this demonstration is a report that details the EMWD-GRS system's overall performance and its performance in delineating the presence or absence of Cs-137 at the SRS F-Arsa Retention Basin. Support documentation strch as copies of logbooks and calibration reports will be inchuded as an appendices to the final report. 
This Page Intentionally Left Blank 


\subsection{REFERENCES}

GeoTrans, Int. 1992. Groundwater Flow Madel for the General Separations Arec, Savannath River Site. GeoTrans Projeet No. 3017-003. Sterling, VA. GeoTrans, Inc.

Leino, R., D.C. George, B.N. Key, L. Knight, and W.D. Steele. 1994. Field Calibration Facitities of Envirommental Measurement of Radizm, Thorizm, and Potassium. Doe/10/12584179; GJ/TMC-01 (Third Edition). Grand Junction, CO. RUST Geotech, Ine.

Westinghouse Savarnah River Company. 1994a. Phase II. Revision I Remedial Investigation Work Plam for the F.Area Retention Basin (281-3F) (O). WSRC-RP-94-498, Rev 1. Aiken, SC. Westinghouse Savannah River Company.

Westirghouse Savannah River Company. 1994b. Radiological Performance Assessment for the E-Area Vaults Disposal Facitity (U). WSRC-RP-94-2J8. Aiken, SC. Westinghouse Savannah River Company.

Westinghouse Savannah River Company. 1995. Qualiny Control Summary Report for the $F$ Area Retention Basin, Phase II Project. ESH-EMS-950563. Aiken, SC. Westinghouse Savannah River Company.

Scott, S.C., T.H. Killian, N.L. Kolb, P. Corbo, and I.W. Marine. 1987. Environmental Information Dacument, Sepgrations, Area Retemtion Basins. DPST-85-693, B.I. du Ponte de Nemours and Company. Aiken, SC. Savannah Rivè Laboratory. 
This Page Intentionally Left Blank 


\section{APPENDIX A}

Background Soil Analytical results

P-Area Retention Basin

(Table 3-2 from WSRC, 1994)

A-1

$-\quad m \cdot$ 
This Page Intentionally Left Blank 
Table 3-2

Eackground Soil Analytical Resolts

F-Area Retention Basin (281-36)

\begin{tabular}{|c|c|c|c|c|c|}
\hline $\begin{array}{l}\text { Sample ID } \\
\text { Facility Jdentifier } \\
\text { Sampling Point } \\
\text { Sample Number } \\
\text { Sampling Inetrvai (it.) } \\
\text { Comment }\end{array}$ & $\begin{array}{c}102200 \\
\text { FRB } \\
01 \\
02 \\
0.5-2.0\end{array}$ & $\begin{array}{c}10202 \\
\text { FRB } \\
01 \\
03 \\
50-7.0\end{array}$ & $\begin{array}{c}102204 \\
F+B \\
01 \\
04 \\
10.0-12.0\end{array}$ & $\begin{array}{c}102205 \\
\text { FRB } \\
01 \\
05 \\
15,0-17.0\end{array}$ & $\begin{array}{c}102206 \\
\text { FRB } \\
01 \\
06 \\
20,0-22.0\end{array}$ \\
\hline 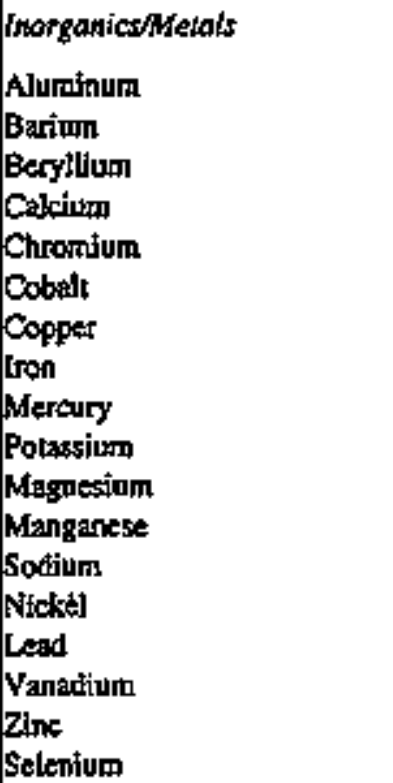 & $\begin{array}{c}(\mathrm{mg} / \mathrm{kg}) \\
9450 \\
29.9 \\
0.251 \\
194 \\
13.6 \\
1.7 \\
2.72 \\
11000 \\
.0688 \\
156 \\
148 \\
150 \\
16.6 \\
3.12 \\
7.39 \\
29.7 \\
5.15 \\
B D L\end{array}$ & $\begin{array}{c}\text { (mg/kg) } \\
15000 \\
27.2 \\
0.236 \\
270 \\
17.8 \\
1.42 \\
4.86 \\
16100 \\
.078 \\
195 \\
247 \\
37.7 \\
24.6 \\
4.24 \\
637 \\
35.6 \\
7.69 \\
3.89\end{array}$ & $\begin{array}{c}(m g / k g) \\
4200 \\
3.22 \\
0.0651 \\
67.4 \\
11.9 \\
0.306 \\
1.49 \\
8370 \\
.0933 \\
36.2 \\
38.4 \\
6.82 \\
15.1 \\
0.953 \\
4.04 \\
29.9 \\
2.3 \\
\text { BDL }\end{array}$ & $\begin{array}{c}(m g / k g) \\
2230 \\
1.43 \\
0.112 \\
30.4 \\
8.32 \\
0.193 \\
0.659 \\
4380 \\
\text { BDL } \\
\text { BDL } \\
18.5 \\
2.89 \\
\text { BDL } \\
0.62 \\
4.73 \\
13.1 \\
0.813 \\
\text { BDL }\end{array}$ & $\begin{array}{c}(m g / k g) \\
1440 \\
0.954 \\
0.0727 \\
16.6 \\
2.08 \\
\text { BDL } \\
0.913 \\
2280 \\
.0255 \\
\text { BDL } \\
7.98 \\
4.29 \\
\text { BDL } \\
\text { BDL } \\
4.12 \\
6.75 \\
1.12 \\
\text { BDL }\end{array}$ \\
\hline 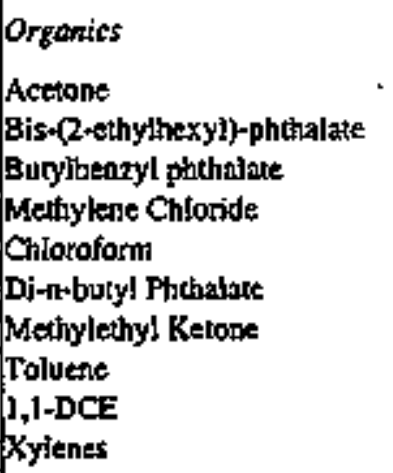 & $\begin{array}{c}(\mu g / k) \\
21.8 \\
12.5 \\
\text { BDL } \\
0.473 \\
0.29 \\
35.7 \\
\text { BDL } \\
0.14 \\
2.12 \\
\text { BDL }\end{array}$ & $\begin{array}{c}(\mathrm{kg} / \mathrm{kg}) \\
5.89 \\
22 \\
\mathrm{BDL} \\
0.708 \\
0.449 \\
48.6 \\
0.326 \\
0.191 \\
\text { BDL } \\
\text { BDL }\end{array}$ & $\begin{array}{c}(1 / \mathrm{g} / \mathrm{kg}) \\
6.75 \\
35.6 \\
\mathrm{BDL} \\
0.67 \\
0.429 \\
472 \\
0.286 \\
0.176 \\
\text { BDL } \\
\text { BDL }\end{array}$ & $\begin{array}{c}(1+g / \mathrm{kg}) \\
6.1 \\
38.4 \\
5.97 \\
1.09 \\
0.477 \\
45.9 \\
0.318 \\
0.182 \\
\text { EDL } \\
0.0795\end{array}$ & $\begin{array}{c}(\mu \mathrm{g} / \mathrm{kg}) \\
4.72 \\
19.8 \\
\mathrm{BDL} \\
0.924 \\
0.402 \\
30.2 \\
0.25 \\
0.13 \\
\text { BDL } \\
\text { BDL }\end{array}$ \\
\hline
\end{tabular}


Table 3-2 (contlaued)

Background Soll Analytical Results

F-Area Retertion Basin (281-3F)

\begin{tabular}{|c|c|c|c|c|c|}
\hline $\begin{array}{l}\text { Sample ID } \\
\text { Facility Identifier } \\
\text { Sampiting Point } \\
\text { Sample Number } \\
\text { Sampling Interval (f.) } \\
\text { Comment }\end{array}$ & $\begin{array}{c}202200 \\
F R B \\
01 \\
02 \\
0.5-20\end{array}$ & $\begin{array}{c}\text { J02207 } \\
\text { FRE } \\
01 \\
03 \\
5.0-7.0\end{array}$ & $\begin{array}{c}10204 \\
\text { FRB } \\
03 \\
04 \\
10.0-12.0\end{array}$ & $\begin{array}{c}10206 \\
\text { FRB } \\
0 \mathrm{~J} \\
05 \\
15.0-17.0\end{array}$ & $\begin{array}{c}102206 \\
\text { FRB } \\
01 \\
06 \\
20.0-22.0\end{array}$ \\
\hline $\begin{array}{l}\text { Radionuelide Indicators and } \\
\text { Radfonuel'ides }\end{array}$ & $(\mathrm{pCl} / \mathrm{g})$ & $(p C \mathrm{v} / g)$ & $(p C \nu)$ & $\left(p C V_{g}\right)$ & $(\rho \mathrm{CI} / g)$ \\
\hline Actinüum-228 & 1.36 & 1.58 & 5.16 & 0.758 & $\$ .14$ \\
\hline Gross Alpha & 8.18 & 20.7 & 152 & 2.44 & 8.86 \\
\hline Americium-241 & BDL & 0.252 & BDL & BDL & BDL \\
\hline Nonvolatile Beta & 8.69 & 12.1 & 6.79 & BDL & 7.18 \\
\hline Carbon-14 & 0.166 & BDL. & BDL. & 0.113 & 0.157 \\
\hline Cesium-137 & 0.0793 & 0.0425 & 0.0116 & BDl. & 0.00622 \\
\hline Europium-155 & 0.103 & 0.0863 & 0.0611 & 0.0405 & 0.0859 \\
\hline Ponassium 40 & 1.16 & 1.33 & 0.634 & 0.704 & 1.04 \\
\hline Manganese-54 & 0.0189 & 0.0282 & 0.0186 & 0.0176 & 0.0183 \\
\hline Nickel-63 & 0.918 & 1.82 & 1.97 & 1.18 & 15 \\
\hline Lead-2J2 & 1.37 & 1.55 & 1.2 & 0.798 & 1.17 \\
\hline Promethith-147 & BDL & EDL & BDL & BDL & 0.0754 \\
\hline Plutonium-238 & BDL & BDL & $\mathrm{BDE}$ & 0,037 & BDL \\
\hline Putonitum-239/240 & 0.284 & 0.107 & 0.12 & 0.178 & 0.321 \\
\hline Ratitrun-226 & 0.445 & 0.557 & 0.442 & 0.264 & 0.224 \\
\hline Radium-228 & 0.633 & 0.842 & 0.831 & 0.55 & 0359 \\
\hline Sironcidra-90 & 0.19 & 0.26 & BDL. & BDL & BDL \\
\hline Thoritm-228 & 1.49 & 1.59 & 1.39 & 0.774 & 1.84 \\
\hline Thorinm-230 & 0.891 & 1.06 & 0.521 & 0.502 & 1.14 \\
\hline Thorium-234 & 1.19 & 158 & 0.807 & 0.74 & 1.14 \\
\hline fTolal Radiutr & 8.7 & 7.8 & 4.7 & 3 & 5.3 \\
\hline Uranitom-235 & 0.0347 & BDL & 0.0422 & BDL & EDL. \\
\hline Jraniक्या-238 & 0.641 & 0.954 & 0.697 & 0.316 & 0.535 \\
\hline Uranium-233/234 & 0.802 & 0.98 & 0.751 & 0,366 & 0.651 \\
\hline Thortam-232 & 1.39 & 1.58 & 1.09 & 0.656 & 1.49 \\
\hline Neptonium-237? & BDL & 0.0647 & 0222 & 0.0459 & BDL \\
\hline Neptunìm-239 & 0.093 & 0.078 & 0.0553 & 0.0366 & 0.0776 \\
\hline Techricium-99 & 1.7 & 1,4 & 1.36 & 0.484 & 0.411 \\
\hline Ytrium-88 & EDL & BDL & BDL & EDL & 0.00541 \\
\hline Proprelhiom-144 & EDL & BDL & BDL. & BDL & 0.00499 \\
\hline Promethium-146 & $B D L$ & 0.0185 & 0.00654 & BDL. & 0.0754 \\
\hline Zirconium-9s & 0.032 & 0.0331 & 0.0177 & 0.0245 & 0.0465 \\
\hline$\dot{0}$ & & & & & \\
\hline
\end{tabular}

$\mathrm{BDL}$ = Not delected above method dexeclion limits 


\section{APPENDIX B}

Quantitative Laboratory Analysis of Soil Samples collected from Sampling

Locations FRB-05, 06, 07, 08, and 19 at the SRS F-Area Retention Basiu

(from Appendix E of WSRC, 1995)

B-1 
This Page Intentionally Left Blank

B-2 
SAMPLE NAME: FRB-05OL

Localion (SRS Coordinares)* 53723 E 76383 N

Sample Marrix: Soil

Constituead

Melol Parmeters

Arsinic, wotal recoverable

Bariun, latal reconerable

Berylliom, total rocowerablat

Chtaminum, cotal recoverable

Nidkel, tolal rtooverable

Lead, tolal recoverabte

Thallinam, rosol cecowerable

Kadiokgrical Parametas

Gross alphe

Amtríciun-241

Bartuon-133

Nopwolatile bela

Caroo-14

Cobalt -60

Cotinga-137

Europiunta+154

lodin-329

Potassium-40

Nitkel-63

Phinilum-25\%R41

R.

Soontium-90

Techmelimar-99

Uyrnitrm-238

Sentivolatile Parameiers

Dikenzetiran

Di-n-bityl phthalate

N-Nituosotiphenylanin:

2-Chloroptenet

Volaile Parameters

Didhloromethane (Methyloge ctibride)

Carbon disulfide

Tolvene

Triehloroethylene
RQ AQ B Result

Sample ID: 10202

Intervat Deptes: 0.00 to J.00

Percent Salids: 90.00

ป

5.85

15.40

0.181

834

1.26

$5 . \$ 1$

U

5.85

$\begin{array}{lll}\text { mgkg } & 5.85 & \text { EPAGO10 } \\ \text { mg/kg } & 2.92 & \text { EPAGO10 } \\ \text { mgkg } & 2.92 & \text { EPAG010 } \\ \text { mg/kg } & 2.92 & \text { EPA6010 } \\ \text { mg/kg } & 2.92 & \text { EPA6010 } \\ \mathrm{mg} / \mathrm{kg} & 5.85 & \text { EPA6010 } \\ \mathrm{mg} / \mathrm{kg} & 5.85 & \text { EPA6010 }\end{array}$

pCils 197

EPLALOOIB

$-0.0 .0 .0 .0101$

$0.001340,0098$

$5.07 \div 2.22$

pCirg 0.06

pCi/z 0.0058

pCits. $\quad 3.99$

$\mathrm{DCS} / \mathrm{q}^{\circ} \quad 0.166$

$\mathrm{PCI} / \mathrm{s} \quad 0.004$ !

pCis $\quad 0.005$

$\mathrm{pCi/s} \quad 0.0213$

pCifs $\quad 0.0983$

$\mathrm{pCu} / \mathrm{g} \quad 0.0379$

pCis 0.767

$\mathrm{pCl} \quad 0.06$

$\mathrm{pC} / \mathrm{s} \quad 0.0455$

$\mathrm{pCi} / \mathrm{g} \quad 0.309$

$\mathrm{pCD} \quad 0.299$

pis 0.05

EPIA-01IB

EPLA-OIBB

EFLA-OOBB

EPLA-003B

EPIA-O13B

EFIA-OLBB

EPLA-013B

EPLA-006B

EPIA-013B

EPLA-0Z2

EPIA-OA2B

EPLA-013B

EPLA-OJ4

EPLA-0OSB

$0.07 \%]+14$. 12

$0.729=0.143$

E[AA-()]JB

rofkg 36.90

Mekg 3690

मBA 36.90

EPASTI

$u$
$u$

U $\quad 36.90$

U 36.90

ne/ks 3690

EPA8270

EPA8270

EPAS270

$\begin{array}{llll}\text { UI } & \text { QO } & \text { H } & 0.556 \\ \text { UJ } & 0 & H & 1.11 \\ \text { UJ } & \text { vo } & H & 0.189 \\ \text { UJ } & 0 O & H & 0.0556\end{array}$

ME 0.556 EPA8260

HAlkg [.]] EPA8E60

$\rightarrow$ H $0.556 \quad$ EPA\$260

㙁 0.0556 EPAR260

F+Ara Ferenion Bssin, Phase il 
SAMPLE NAMA: FRB-OSOZ

Localion (SRS Coordinales): 53727 E 76383 N

Sample Malvix Soil

Canstituept

Metsal Parameters

Asseniti, tolal recoveratile

Barium, lotsil recoverable

Beryllinin, totnl recoverstle

Chromium, tocad toctiversid:

Nhekel, total recowerable

Lead, worat recoverable

Thallinm, total raxoverable

Radiologieal Pardmeters

\section{Grabs alpha}

Americium-241

Earium- 133

Nomolatile bela

Catboo-14

Cobstit-60

Cesingo-137

Europium-154

Iadine 129

Ponssium-40

Nick

PIutonism-729/240

Radiam-2z6

Suroodism-90

Tecthnetiurr- 99

Urmintra-238

Semirolatite Paraneders

Dibenzofaran

Di-zi-botyl phuthatate

N-Witrosodiphesylemine

2-Chlorophenol

Volatile Parameners

Dietiloromethars (Molhylene chtoride)

Carbatr disalfide

Toluene

Trichlopocthylene
RQ AQ B Restlt Uth D.Limit Method

$\mathbf{u}$

5.91
23.10
0.238
12.79
3.13
4.40
5.91

mink 5.91

$\pi$ oks 296

moks 2.96

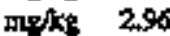

melkg 296

ग18kg 5.91

mgerks 5.91

EPÁ́OLO

EPAGOLO

EPA6010

EPA6010

EPA6010

EPA6010

EPASOLO

\begin{tabular}{|c|c|c|c|}
\hline 4.57+2.11 & $\mathrm{pCi/s}$ & 2.61 & EPLA-00]B \\
\hline $0.0237 \pm 0.0242$ & pCifg & 0,06 & EPLA-01]B \\
\hline$-0.012 \pm 0.0039$ & pcisis & 0.006 & EPLA-0]3B \\
\hline $6.20+2.41$ & $\mathrm{pCig}$ & 4.22 & EPIA-00JB \\
\hline$-0.0028=0.0864$ & pCs's & 0.167 & EPIA-0,03: \\
\hline $0.0005=0.0026$ & $\mathrm{p} / \mathrm{g}$ & 0.0045 & EPIA-013B \\
\hline $0.0903-0.006 ?$ & pciss & 0.005 & EPLA-013B \\
\hline$-0.0009 \times 0,0 \geq 14$ & PCis & 0.023 & EPA-013B \\
\hline $0.0079=0.046$ & pCis & 0.0931 & EPLA-006B \\
\hline $0.936 \pm 0.0687$ & $\mathrm{PC} / \mathrm{s}$ & 0.0391 & EFIA-013B \\
\hline $0.712 \div 0.369$ & $\mathrm{pcts}$ & 0.736 & $E P H A-O 2$ \\
\hline $0.0008+0.0332$ & $\mathrm{pCi}$ & 0.0754 & $E P / A-012 B$ \\
\hline $0.883 \pm 0.0994$ & pCitg & 0.0687 & EPLA-013B \\
\hline $0.0713 \pm 0.168$ & $\mathrm{pCi} / \mathrm{s}$ & 0.26 & EPIA-O04 \\
\hline $0.0212+0.109$ & pCi & 6.254 & EPIA-00SB \\
\hline $0.72=0.156$ & $\mathrm{CO}$ & 0.06 & EP[A-01)B \\
\hline
\end{tabular}

634

1830

7.09

7.84

$\mu / \mathrm{kg} \quad 37.30$

$\mu \mathrm{Nkp} \quad 3730$

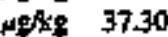

$\mu \mathrm{B} / \mathrm{kg}$ 37.90

EPA\$270

EPA 8270

EPA8270

EPA8Z70

\begin{tabular}{|c|c|}
\hline UJ & 0,562 \\
\hline UJ Q & 3.12 \\
\hline VQ & 0.169 \\
\hline C & 0.0562 \\
\hline
\end{tabular}

refks

0.562 EPA8260

$20 \mathrm{~kg} \quad 1.12$ EPA8260

0.562 EPA8260

$M 9 \mathrm{~kg} \quad 0.0562$ EPA\$260

F-Area Rutention Basin, Phase II 
SAMPLE NAME: FRP-0503

Lostion (SRS Coordinates): 53723 E $76383 \mathrm{~N}$

Sample Marix: Soil

Constittent

Mecal Parmerers

Arsenit, totel recoverable

Banium, 10tis recoverable

Berylliura, sotal recovetrable

Chrositum, total recoverablo

Nitikel, total secoverable

Lesil, total reconerable

Tnatlinen, total tecovereble

Radiologieal Parsacers

Gross alpha

Americitur-241

Bariura+i33

Nonvelatile bets

Carboa-14

Cobel1-60

Cestum-137

Europinum-154

lodine-12

Posatsivin-40

Niekel-63

Piusonjarm-239/240

Radiun-226

Simotrivem-50

Teciuletium +99

Unaium-238

Semiroiatile Paraneticts

Dibenzofiusen

Dith-buigl philalate

N-Nicrosodipikalylamine

2-Chlocophenot

Volabile Parameters

Cichloromethane (Melinylene chioride)

Cribon disulifide

Taluepe

Trichloroethytenc
RO AO $\mathrm{g}$

Lterval Depils: 8.00 io 10.00

Percent Solids: 78,90

U

6.75

6790

0.645

9.99

490

431

U

6.75

$1550=3.72$

$0.0153=0.0225$

$0.003 \div 0.0069$

$17.40 \pm 3.34$

UI $\quad 0.059 \div 0.058]$

U] $-0.0013 \div 0.003$

$5.48 \pm 0.034 \mathrm{I}$

uj

บ1

vi $v$

U]

J $c$

บ]

$-0.0348 \div 0.0177$

$0.0064 \pm 0.059$

2.49:0.10]

$0.669=0338$

$0.0752 \div 0.0459$

$0.967 \pm 0.0891$

9.99*0.49

$-0.091=0.117$

$0.974 \pm 0.184$

pCiss 219

pcivg. 0.06

PCis 0.0102

p.iph 4.56

pCifs 0.192

pCtis 0.0049

oCis 0.0075

$\mathrm{pCU} \quad 0.0247$

pCis 0.122

pcis 0.0404

pCifg 0.672

pCi's 0.05

pCis 0.0645

pCits 0.418

pCirg 0.368

pCis 0.06

EPA 6010

EPA6050

EPA6010

EPA6010

EPA6010

EPA6010

EPASOID

EPLA-COIB

EPLA-0]1B

EPLAt013B

EPLA-0O1B

EPLA-003B

EPLA-013B

EPLA-013B

EPLA-0]3B

EPLA-006B

EPLA-013B

EPIA-BDZ

EPLA-0]2B

EPLA-013B

EPLA +004

EPLA+005B

EPIA-OIJB

HSA 4260 EPA8270

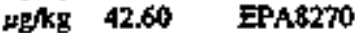

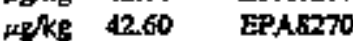

He/kg 42.60 EPA8270
8.09
42.60
42.60
4260

0.436

I $\mathrm{OB}$

U) 0

U] vo

UJ 0

128

0.282

0.0641

$\begin{array}{lll}\omega \mathrm{g} / \mathrm{kg} & 0.641 & \text { EPA8260 } \\ \mathrm{HE} / \mathrm{kS} & 1.28 & \text { EPAB260 } \\ \mu \mathrm{g} / \mathrm{kg} & 0.641 & \text { EPA8260 } \\ \mathrm{tg} / \mathrm{kg} & 0.0641 & \text { EPA8260 }\end{array}$

F-Area Retentor Basin, Phase if 
SAMGLE NAME: FRB 0504

Locrtion (SRS Coordinrtes) 53729 E 76383 N

Sanple Matix: Soil

Constitumnt

Metal Parameters

Asșenic, totaj recoverable

Burism, total recowerable

Beryilium, toral recoverabis

Chromiurn, zolal necowerabie

Nickel, zotsl recowerable

Lead, tout recowerable

Thallinut, rotgl recoverable

Radiological Paraptelers

Gross alphe

Amtricivin-241

Barium-133

Nonvolatile belo

Cutbon-14

Cobsili-60

Cestum-137

Encopiuna*]S4

jodint-129

Potastiun -40

Nickel-63

Pintoding-239/240

Radium-276

Sumalum-90

Technotiure-9s

Urantum-23:3

Semirolatie Faranesers

Dibenzofuran

Di-n-buryl phlhatale

it-kitixosodipheaylawire

2-Chlarophand

Volarik Paramelers

Diehloromethane (Melhylene chloride)

Carbon disulitide

Toluene

Jrichloroethylene
RQ $A Q$ a Result

Sarple ID: 1027

Interval Daphis: 10.00 to 12.00

Percent Solids: 86.00

$\mathrm{U}$

6.12

30.50

0.3]

11.80

2.69

4.75

3.46

$m g / k \mathrm{~g} \quad 6.12$

magkg 3.06

$\mathrm{mg} / \mathrm{kg} \quad \mathbf{3 . 0 6}$

IDgike 3.06

$\mathrm{mg} / \mathrm{kg} \quad 3.06$

$\mathrm{mm} / \mathrm{kg} \quad 6.12$

mofkg 6.12

EPA6010

EPA6010

EPAON10

EPA6010

EPA6910

EPA60IO

EPh6010

c)

UI

$14.600=3.67$

PCi/s 2.19

EPLA-003B

0.025620 .0243

$-0.00 \leq 0.0104$

$229.00=10.10$

(u)

$0,043,00,0882$

$0.0318 \pm 0.0063$

$11,20=0.0474$

$-0.0204 \pm 0.0211$

$0.058 \pm \pm 0.10$

1.950.0.0979

$2,06=0,433$

Dis 0.06

APIA-OI IB

$\mathrm{pCi} \quad 0.0147$ EPLA-0[3B

DCi's 4.04 EPLA-00IB

PCig 0.774 EPIA-005B

pCis 0.0055 EPIA-OLIB

pCirs 0.0093 EPLA-0L3B

pCi's 0.0300 EPLA-0t3B

pCiks 0.187 EPLA-006'

PCive 0.0484 EPLA-013B

pCV 0.75 EPLA+027

pCis 0.0663 EFL-012R

U1 $0.0091 \pm 0.03$

J C $0.809 \pm 0.0774$

$385.00 \pm 9.59$

UI

$0.243 \pm 0.11$

$0.84] \pm(1.153$

pCV/g $\quad 0.0578$

$\mathrm{pCig} \quad 2.09$

$\mathrm{pCH} / \mathrm{0.30}$

EFIA-013B

EFLA-004

EPLA-C05B

pCis 0.06

EPLA-011B

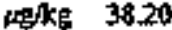

$50 / \mathrm{kg} \quad 3820$

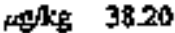

PA8270

3820

38.20

38.20

Hofk 38.20

EPA8270

EPA8270

$\begin{array}{lll} & & 0.36 \\ U & v & 1.16 \\ U & & 0.36 \\ & & 0.0581\end{array}$

mosk molks

Atwik.

$\mu \mathrm{g} / \mathrm{kg}$
0.581

2.16

0.581

$0.05 \$ 1$
EPA8260

EPA8260

EPA8260

EPA 8360

F.Arta Retemion Basin, Phase If 
SAMP1ENAME: HRB-050S

Locrlion (SPS Coordtuales): 53723 E. $76383 \mathrm{~N}$

Sample Matrix: Soil

Cosstituent

Metal Parametass

Arsenic, tolai recoverable

Barism, total rocovitalole

Berylfilm, 1001 tecoverable

Chromiura, total recaverable

Nickel, tokal recoverable

Lexd, lotnd recowcrable

Thallitual, tobl recoverabse

Radiological Paramears

Gross aiphs

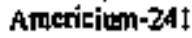

Bsriom-133

Nonvodaile beta

Carban-14

Colas $(-60)$

Cestum-137

Europiurn-154

Lodins i29

Fotsssium-40

Nicked 63

PJutonitum-239/240

Rediuen-206

Stromintm-90

Tedunctiun-99

Uraium-238

Semivalatile Parameners

Dibenzoringean

Di-n-buty's phthaialt

N-Ninosodiphenylumine

2-Chlorophenot

Volatile Parameas

Dishloromethane (Medhylene chloride)

Carben disulude

Tolume

Trichlotpethyiene
RQ $A Q$ B Result

v

$$
\begin{gathered}
6.19 \\
17.10 \\
0.189 \\
14.20 \\
2.85
\end{gathered}
$$

$\mathbf{u}$

531

\begin{tabular}{|c|c|c|c|c|}
\hline \multirow{3}{*}{ UI } & $17.80=4.15$ & pClis & 2.11 & EPLA+001B \\
\hline & $-0.0023 \pm 0.0118$ & $\mathrm{pC} \mathrm{V}_{\mathrm{B}}$ & 0.06 & EPLA-0IIB \\
\hline & $-0.145 * 0.10]$ & $\mathrm{pOS}$ & 0.0174 & EPIA-0138 \\
\hline & $302.00=11.30$ & pCisg & 3.98 & EPIA-0OJB \\
\hline \multirow[t]{3}{*}{ UI } & $-0.0186=0.0863$ & $\mathrm{pCO} / \mathrm{s}$ & 0.136 & EPLA-003B \\
\hline & $0.0354=0.0081$ & $\mathrm{PCO} / \mathrm{B}$ & 0.0063 & EPIA-0:3B \\
\hline & $931=136$ & pCits & 0.0124 & EPLA-OJ3B \\
\hline & $0,005 \leqslant 0,0059$ & poing & 0.0384 & EPIA-OI3B \\
\hline & $0.0388 \div 0.162$ & pCists & 0.30 & EPLA-0196B \\
\hline \multirow[t]{3}{*}{ I } & $1.23 \pm 0.16$ & $\mathrm{pC} / \mathrm{g}$ & $0.0 \leq 74$ & EPAA-013: \\
\hline & $199=0.458$ & pcis & 0.811 & EPLA 072 \\
\hline & $+0,0066=0,0329$ & pcisg & 0.0821 & ETA-012B \\
\hline \multirow[t]{3}{*}{$c$} & $0.821 \pm 0.102$ & $\mathrm{pCl} / \mathrm{s}$ & 0.0678 & EPLA-B]3B \\
\hline & $804,00 \pm 9.06$ & pCisg & 0.786 & EPLA-004 \\
\hline & $0.161 \pm 0.192$ & $\mathrm{pCI} / \mathrm{s}$ & 0.54: & EPLA-005B \\
\hline & $1.02 \pm 0.20 ?$ & & 0.0739 & $E F[A-0] 1$ \\
\hline
\end{tabular}

6.19

Sagnple FD: 102275

Inierml Depths: 12.00 to 14.00

Percent Solids: 85.00

$\begin{array}{ll}\text { U } & 39.10 \\ U & 39.10 \\ U & 39.10 \\ U & 39.10\end{array}$

400/ke 39.10

39.10

aglks 39.10

agly 39.50

EPA8270

EPA2270

EPA8270

EPAKITO

$\begin{array}{llll}\text { U } & 0 & \text { H } & 0.588 \\ \mathbf{U} & 0 & H & \mathbf{H} \\ \mathbf{U} & \mathrm{VO} & \mathrm{H} & 0.165 \\ \mathbf{U} & 0 & \mathrm{H} & 0.0588\end{array}$

$\begin{array}{lll}10 / \mathrm{kg} & 0.588 & \text { EPA8260 } \\ w \mathrm{~kg} & 1.18 & \text { EPAS260 } \\ \mathrm{w} / \mathrm{kg} & 0.588 & \text { EPA8260 } \\ \mathrm{w} / \mathrm{kg} & 0.0588 & \text { EPA8260 }\end{array}$

F-Area Retention Basio, Fhase II 
SAMPLE NAME: FRB-0506

Loesion (SRS Coordinates): 53723 E $26383 \mathrm{~N}$

Sample Matrix: Soil

Copstituent

Metal Parametas

Arsenic, total recoverable

Barion, Lotal Jecowerabj:

Beylium, total recoverabls

Chromfum, total recoverabie

Nickel, zotad recowatale

Led, told recoverable

Thallium, total recoverable

Radiologieal Parameters

Gross alpha

Anerik

Bariurs-133

Nonvalatle beta

Carbon-14

Cobali-60

Cesiner-137

Exropium-154

Iodine-129

Pobasiun -40

Nickel-63

Putminin-239/240

Radivan-226

Sropium-99

Techoretion-99

Ijrantaum-238

Sernivolatile Paramerters

Dibenzofuran

Di-n-butyl phthalate

N.Nirrosodtohenylamine

2-Chikrophenol

Volstile Parameters

Diebloromertane (Methylene chioride)

Cintoin dispulfide

Toluene

Tsidhiorocthylene
Sample 1D: 10276

lnierval Depths: 34,00 to 16.00

Percem Solids: 83.00

RQ AQ B Resoll

Uait D.Litt Method

U

6.34

9.46

0.139

54.00

2,26

6.58

v

6.34

$\begin{array}{lll}\mathrm{mg} / \mathrm{kg} & 6.34 & \text { EPAGDIO } \\ \mathrm{mg} / \mathrm{kg} & 3.17 & \text { EPA6010 } \\ \mathrm{mg} / \mathrm{kg} & 3.17 & \text { EPAGO10 } \\ \mathrm{mg} / \mathrm{kg} & 3.17 & \text { EPA6010 } \\ \mathrm{mg} / \mathrm{kg} & 3.17 & \text { EPA6010 } \\ \mathrm{mgks} & 6.34 & \text { EPA6010 } \\ \mathrm{mg} / \mathrm{s} & 6.34 & \text { EPA6010 }\end{array}$

$14.60 \pm 4.56$

$0.0133=0.0247$

(i) $\quad 0.0014 \pm 0.0075$

บ)

$0.0263 \pm 0.0873$

$0.01 \pm 0.0056$

$3.40 \div 0.0265$

[1

U1]

U] $v$

$0.00003 \pm 0.0302$

$0.0361 \pm 0.194$

I. $14 \pm 0.0839$

$1.19 \pm 0.41$

UI $0.0352 \pm 0.0353$

$0.919 \pm 0.208$

$328.00 \pm 11.50$

U1

$-0.0358 \div 0.0528$

I.03 $=0.241$

$\begin{array}{ll}\text { poifg } & 3.6 \\ \text { pCisg } & 0.0 \\ \text { pCigg } & 0.0\end{array}$

EPIA-00IB

EPLA-011B

PCig 0.0114 EPIA-013B

PCis 4.14 EPIA-ONIB

$\mathrm{pCi} / \mathrm{g} \quad 0.1 \mathrm{~B}$ EPIA-003B

$\mathrm{PC} / \mathrm{s} \quad 0.0053$ EPLA-0I3B

$\mathrm{PCi} / \mathrm{g} \quad 0.0082$ EPIA-013B

$\mathrm{pCi} / \mathrm{s} \quad 0.0314$ EPLA-013B

$\mathrm{PCV} / \mathrm{g} \quad 0368$ EPLA-006B

pCitg 0,0493 EPLA+Ot3B

pCitg 0.778 EFIA-022

PCils $0.06 \quad$ EPIA-012B

pCi's 0.10 EPUA-013E

WCU/ 1.79 EPLA+104

pCits 0272 EPIA-00SB

pCits 0.0915 EPLA-0118

$\begin{array}{ll}u & 39.70 \\ \mathbf{v} & 39.70 \\ u & 39.70 \\ w & 39.70\end{array}$

Hefse 39.70

EPA $\$ 270$

$\mu g / k g \quad 39.70$

melke 39.70

EPA8270

EPA\$270

Mg/kg 39.70 EPA8270

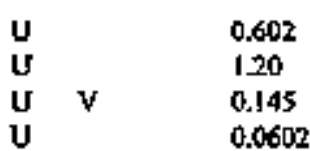

म상

$4 \mathrm{~kg} 120$ EPA8260

rofks 0.602 EPA\$260

LOKE 0.0602 EPA8260

F-Ase Retention Brsin, Phase It 
SAMPLE WAME: FRE-0507

Lacation (SRS Coordinatis)t $59723 \mathrm{E} 76383 \mathrm{~N}$

Sample Matofx: Soil

Constituent

Metal Paramelers

Arsenic, Lotal recoveroble

Barium, total recoverable

Beryllium, totat recowerable

Clrowium, iotal recoverable

Nickel, total recortable

Lead, wotal rstanterible

Ttaillium, cotal recoverable

Radiological Paramelets

Gross alpha

Americtom-2\%1

Barium-133

Nonvolatile bev

Carbon-14

Cobah-60

Cessiun-137

Europintm+154

Lodine-129

Potassium-40

Nicket-65

Photontumi-2391240

Radium-226

Srontîum-90

Tectutelium-99

Uranhum-238

Servivolalile Parametiers

Dibenzofuran

Diegebuteyi phtialate

N-Nitrosodiphenytamine

2-Chilowphenol

Vohdile Parameters

Diebleromethane (Methylene ditaride)

Carban dFtrifide

Tolueas

Trichlordecthylene
RQ AQ B Result

Unit

D.Limit

Methad

U

6.58

8.18

0.15

15.40

2.35

v

5.66

6.58

Sarpple 1D: 102278

Interval Depches $26.00 \mathrm{to} 18.00$

Pereent Solids: 80.00

sult

(n)

$\begin{array}{lll}\text { mejkg } & 6.58 & \text { EPA6010 } \\ \text { mg/kg } & 3.29 & \text { EPA6010 } \\ \text { mg/kg } & 3.29 & \text { EPA6010 } \\ \text { mejks } & 3.29 & \text { EPA6010 } \\ \text { mg/kg } & 3.29 & \text { EPA6010 } \\ \text { madkg } & 6.58 & \text { EPA6010 } \\ m g / k g & 6.58 & \text { EPA6010 }\end{array}$

\begin{tabular}{|c|c|c|}
\hline & & $17.80=4.71$ \\
\hline UI & & $0.0131 \pm 0.0152$ \\
\hline Ul & & $\begin{array}{l}-0.0276 \pm 0.0058 \\
125,00 \div 7,75\end{array}$ \\
\hline UI & & $-0.0581=0.0843$ \\
\hline UI & & $\begin{array}{c}-0.0003 \pm 0.0037 \\
0.261 \pm 0.0097 \\
0.0403 \pm 0.0253\end{array}$ \\
\hline ut & & $0.9875 \div 0,07$ \\
\hline UI & $v$ & $\begin{array}{l}1.16 \pm 0.0972 \\
1.35=0.398\end{array}$ \\
\hline U| & & $0.0000=0.0124$ \\
\hline $\mathbf{J}$ & c & $\begin{array}{l}0.899 \pm 0.0842 \\
81.50 \pm 1.19\end{array}$ \\
\hline UI & & $\begin{array}{l}0.0179 \pm 0.113 \\
0.873 \pm 0.176\end{array}$ \\
\hline
\end{tabular}

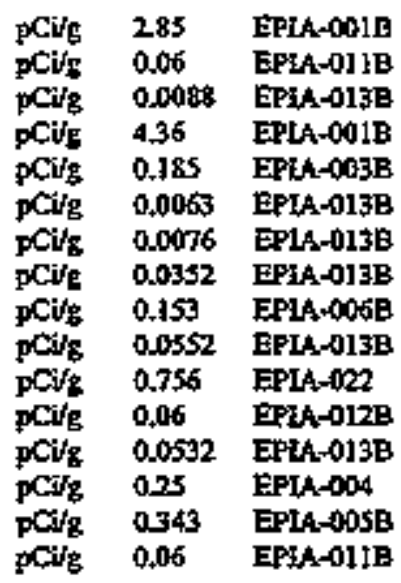

HEk8 $4130 \quad$ EPA8270

IIfks 4130 EPAB270

$\mu$ E $/ \mathrm{kg}$ 4!,30 EPA8270

HEKS 4E30 EPAB270

$$
\begin{aligned}
& 41.30 \\
& 41.30 \\
& 4 \pm .30 \\
& 41.30
\end{aligned}
$$

$\begin{array}{lll} & & 0.488 \\ & & \\ \text { U } & v & 1.25 \\ & & 0.275 \\ & & 0.075\end{array}$

$\mu \Leftrightarrow$

нu/kg 12

HWh 0.0025
EPA8260

EPA8260

EPA8260

EPA8260

F-Arez Reiention Basin, Pase If 
SAMPLE NAME: FRE-0S0S

1oction (SRS Coordirntes): 53723 E $76383 \mathrm{~N}$

Sample Maurix: Soil

Constitueta

RQ AQ $\mathrm{A}$ Result

v

Ansentic, total recovernble

Barium, total recorverablt.

Beryllium, noth recoverable

Chromium, tolal recoverable

Nickell, toral recoverabte

lead, cotal recoverable

Thallium weal recoveratile

Radiological Paramelurs

Gross alphe

Americiurr-241

Brarium-J35

Norvolatile beta

Carbor-14

Cobsil-bon

Cesium-137

Europiun-154

Iodion-129

Potstgimm-60

Wickel-63

Plutonism-239240

Radiur-276

Suontion-90

Technetiug-99

Uranium-238

Semivolatile Faramelers

Dibenzofurang

Di-n-burgil phliatace

N-Nitrosodiphenylanine

2-Chlorophenol

Volatile Prameters

Dichlosomelhane (Meinylene chloride)

Carbon đisulfide

Toluente

Trichloroetinylene

$$
\begin{array}{r}
41.40 \\
41.40 \\
41.40 \\
4.54
\end{array}
$$

\begin{tabular}{|c|c|}
\hline & $\begin{array}{l}15.80=439 \\
0.0206=0.0211\end{array}$ \\
\hline & $\begin{array}{l}-0.0105 \pm 0.005 \\
21.60 \pm 5.58\end{array}$ \\
\hline & $\begin{array}{r}-0.0719=0.0857 \\
0.0008=0.0032\end{array}$ \\
\hline & 0.12350 .0078 \\
\hline & $-0,0093=0.0208$ \\
\hline & $-0.0335 \div 10.066$ \\
\hline$v$ & $1.05 \pm 0.0894$ \\
\hline & $129 \pm 0.452$ \\
\hline & $0.0172 \div 0.0454$ \\
\hline c & $0.954 \pm 0,113$ \\
\hline & $\begin{array}{l}13.00+0.574 \\
0.0849 \pm 0.105 \\
0.979 \pm 0.593\end{array}$ \\
\hline
\end{tabular}

Sample 10, 107279

Interval Depths: 18.00 to 20.00

Percent Solids: 80,60

$\mathrm{pCt} / \mathrm{s} \quad 2.94$

EPLA-001B

pCis 0.06

pCis 0.0079

pCls 423

BLA-0J]E

EPIA-013B

EPJA-0OLB

DCH 0.189 EPIA-003B

pCis 0.0056 EPIA-013B

xCIf 0.0069 EPIA-013B

DCD/2 0.0305 EPLA-013E

$\mathrm{PC} / \mathrm{g} 0.146$ EPLA-0068

pCists 0.0508 EPIALDI3E

PCSI 0.859 EPLA-022

$\mathrm{pC}$ W/ 0.0886 EPIA-012B

PCW 0.0718 EPIA-OI3B

$\mathrm{pCD}$ 0.3II EPIA-004

$\mathrm{pCl} / \mathrm{s} 0.316$ EPLA-OOSB

$\mathrm{pCt} / \mathrm{s}$ 0.16 EPLA-0)IB

$\begin{array}{lll}\mu \mathrm{g} / \mathrm{kg} & 4 \mathrm{~L}, 40 & \text { EPA8270 } \\ \mu \mathrm{g} / \mathrm{kg} & 4 \mathrm{t}, 40 & \text { EPA8270 } \\ \mu \mathrm{g} / \mathrm{Kg} & 4 \mathrm{~L}, 40 & \text { EPA8270 } \\ \mu \mathrm{kg} & 41.40 & \text { EPA8270 }\end{array}$

upks 41.40

EPA8200

F-Ares Retention Brsin, Thase D

$\begin{array}{lll}\mu 4 k s & 0.625 & \text { EPA8260 } \\ \mu / k g & 125 & \text { EPA2260 } \\ \mu \& 1 k g & 0.625 & \text { EPA8260 } \\ \mu g / k g & 0.0625 & \text { EPAB260 }\end{array}$


SAMLLE NAMIE: FRB-0509

Locotion (SRS Coodinales). 53723 E 76383 N

Sample Matrix; Sojl

Constituent

RQ AQ $\mathrm{E}$ Restll

Sample ID: 107288

Interval Depths: 20.00 to 27.00

Percent Solids 79.00

Melal Parameders

Asscenic, solal recoverable

Barjur, colal recoyerable

Beryllinam, utal Jepoverable

Chrominm, local recoveribje

Nickel, rocal recorverablts

Lend, lolil recoveratle

Thalliurs, nol fecoverable

Rodiological Parameacrs

Gross atpha

Anericlum-24 I

Baríum-13s

Nomvolaile bela

Carbon-14

Cobsk-60

Cosisun-137

Europimir 154

Iodion-129

Potas :

Nickele-63

Plulonisu-239/240

Redium-276

Strontutam-90

Technetturn-99

Uramium-238

Semivobatilt Parameters

Diberotoran

DF-buryl ghlusglate

NFNitasodifhenyiamine

2-Chlorephenol

Yolatile Paravetcrs

Dichlow whelhane (Medkylene chloride)

Curban disulfide

Toluens

Trichloroelhylepe

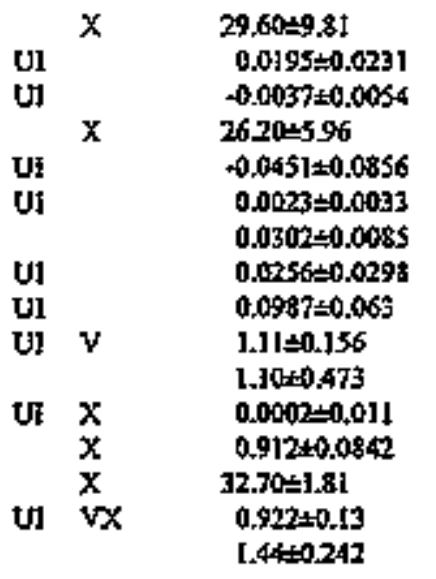

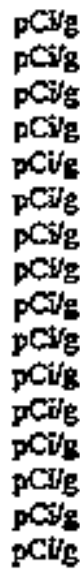

6.74

0.06

0.0078 EPLA-013E

724 EPLA-001B

0.189 EP1A-003B

0.0058 EPLA-013B

0.0068 EPIA-013B

0.0308 EPLA-013B

0.14 EPLA-006B

0.0545 팟-013B

0.924 EPIA-022

0.06 EPIA-012B

4.0576 EPLA-013B

\$.18 EPIA-004

0,368 . EPLA-005B

0,0696 EPIA-011B

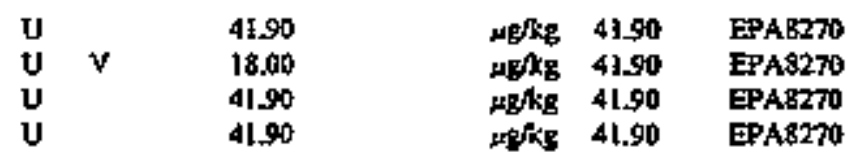

$\begin{array}{llllll}U & & 0.653 & \mu g / k g & 0.633 & E P A 6260 \\ U & & 1.27 & \mu g / k g & 1.27 & \text { EPA8260 } \\ U & v & 0.177 & \mu g / k g & 0.633 & \text { EPA8260 } \\ U & & 0.0633 & \mu g / k g & 0.0633 & E P A 8260\end{array}$

F-Area Relention Basin, Phase ]] 
This Page Intentionally Left BJank.

B-12

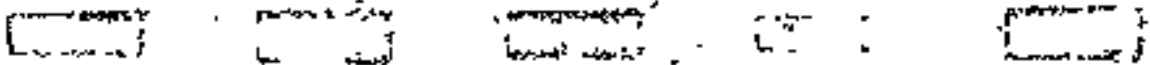


SAMPLE NAME: FRB-0601

Loction (SRS Coordinates): 53775 E $76260 \mathrm{~N}$

Sample Matrix: Soit

Constrituend

Motal Paramettrs

Arsenit, rost recoverable

Barium, rolal recoversble

Bayllium, lotal recoverable

Chrototum, total recoverable

Niekel, tobat recowerable

Lead, lotal recoverable

Thallitun, totsl racow trabte

Radrologieal Parameiers

Gross alpha

Americium-241

Barfum-133

Nonvolecile bets

Carbon-14

Cobalt-60

Cesium-137

Europium-154

Iodiat 129

Potarsiurs-40

Nicked-63

Plotontum-239040

Ridium-226

Strontbum-90

Technetium-99

Uranium-238

Seralvolacile Parmeters

Dibengofiuran

Difr-botyl phthalate

N-Witrosodiphenylamine

2-Chlorophengl

Valofile Paramelers

Dichloramethats (Methylkie chlocide)

Crabon disultide

Tolneare

Triehtoroethylene
RQ AQ B Result Unit D.Limt Mothod

Sample ID: 102249

Intervit Depths: 0.00 to 1,00

Parcent Solits: 9200

U $\quad 5.72$

23.20

0235

8.72

1.79

6.47

$\mathbf{U}$

5.72

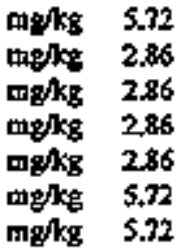

EPA6010

EPA6010

EPA6010

EFA6010

EPAG010

EFA6010

EPAG910

\begin{tabular}{|c|c|c|}
\hline & & $6.88=751$ \\
\hline $\mathrm{Jt}$ & & $0.0144 \pm 0.0 \mathrm{~ms}$ \\
\hline$y$ & & $-0.0021 \pm 0,0048$ \\
\hline & $x$ & $10.10=266$ \\
\hline Ut & & $+0.553+0.102$ \\
\hline UIJ & $x$ & $\begin{array}{r}-0,0019=0.003 \\
0.246 \pm 0.0084\end{array}$ \\
\hline UI & & -0.00790 .0183 \\
\hline זרי & & $0.0141 \pm 0.049$ \\
\hline v| & $v$ & $1.64 \pm 0.4946$ \\
\hline$\downarrow$ & $x$ & I. 1400.408 \\
\hline UI & & $0.0009+0.0245$ \\
\hline$\xi$ & C & $0.805+10.0980$ \\
\hline & $\mathbf{x}$ & $0.346 \leq 0.23$ \\
\hline UIf : & $x$ & $\begin{array}{c}0.0076 \pm 0.569 \\
0 \leq 98 \neq 0.16\end{array}$ \\
\hline
\end{tabular}

\begin{tabular}{|c|c|c|}
\hline$C i / s$ & 229 & $001 B$ \\
\hline & 0.0432 & bls \\
\hline & 0.0068 & 013B \\
\hline & 3.85 & EPIA+ONTB \\
\hline & 0213 & FPPIA-003B \\
\hline & 0.005 & $E P 1 A-013 B$ \\
\hline & 0.0058 & EPLA-013B \\
\hline & 0,0263 & EPLA,0]3B \\
\hline & 0.097 & 톡A-006B \\
\hline & 0.0483 & EPLA-013B \\
\hline & 0.779 & EPAA-022 \\
\hline & 0,0783 & $E P(A+0) 2 B$ \\
\hline & 0.0643 & tepl-0)3B \\
\hline & 0.331 & $E P L A=004$ \\
\hline & 0.428 & EPIA-005B \\
\hline & $0,069 t$ & EP'A-01I \\
\hline
\end{tabular}

$209 / \mathrm{kg} \quad 36,10$

45/kF 36.10

4 Ekg 36.10

EPA8270

FPA8270

EPA\&270

36.10

36.30

wkE $\mathbf{3 6 . 1 0}$

EPA8770

$\begin{array}{llll}\text { J } & Q Q & \text { H } & 0.935 \\ \text { UJ } & Q 0 & H & 1.09 \\ \text { UJ } & \text { vQ } & \text { H } & 0.228 \\ \text { UJ } & Q 0 & \text { H } & 0.0543\end{array}$

rofkg DSA3 EPA8260

4. 109 EPA 2.0926

rAfkg 0.543 EPA8260
$10 \mathrm{~g} / \mathrm{kg} 0.0545$ EPAB260

F-Area Retertion Besin, Phase Il 
SAMPLE NAME: FRB-06O2

Locrition (STRS Coordinates): 5377s E 76260 N

Sample Matrix: Soil

Constitant

Metal Faramelers

Assetic, wol recoverabite

Batim, cotal racoversble

Beryilinem, total recoverible

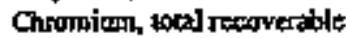

Niekel, total recoverabls

Lead, cocal recoverable

Thallimrs, total recoverabte

Radiological Parameters

Gross elpht

Amsericiura-24!

Barium?139

Nomvolatife bets

Curborl-14

Cobsilt-60

Cesintin-137

Europioum-154

Iodine-129

Pocossingr-40

Nickel-6s

Plutoniterm-239240

Radium-206

Stroptintm-90

Texhnetium-99

Urangump-238

Somivolatile Parminelus

Diberzotiuran

Dis-nbuxyl ptothatate

N-Nirosodiphemylamine

2-Chloroplienol

Volatile Parametiss

Diabloromedhane (Mienylene chloride)

Carbon disulfide

Taluede

Trichloroedhylent
$\mathrm{RQ} A \mathrm{~B}$

$\mathbf{U}$

6.05
26.20
0.238
10.20
1.66
7.22
6.05

if

6.05

$5.67 \leq 2.25$

$-0.4096 \pm 0.0203$

$0.0032 \div 0.00$ d4

$5.05+2.39$

$-0.628=0.101$

$-0.0003 \pm 0.0026$

$0.455 \pm 0.0102$

$0.0031 \pm 0.021$ I

$-0.046 \$ 0.04]$

L.13니,073]

$0.919 \pm 0.393$

$0.1557 \pm 0.0472$

0.7180 .0601

$0.571 \pm 0.275$

$0.0343 \pm 0.103$

$0.688=0.176$

$p C i / s$
$p C t / s$
$p C i / s$
$p C i / s$
$p C i / s$
$p C i / g$
$p C i / s$
$p C i / s$
$p C i / s$
$p C i / g$
$p C i / s$
$p C i / s$
$p C i / g$
$p C i / s$
$p C i / g$
$p C i / s$

207

0.0558

0,0064

4.44

0,225

0.0044

0,0053

0,0029

0.0808

0,0401

0.768

0.0665

0.0431

0,313

0286

0.0541

EPIA-OOAB

EPLA-0] IE

EPLA+0]3B

EFTA-0.0IB

EPPIA-009B

EPLA-0]3B

EPLA-013B

EPLA-0]3B

EPLA-CDEB

EPLA-013B

EPLA-022

EPAA-012B

EPLA-013B

EFWA-004

EPLA-005B

EPLA-0)tB

F-Arta Retertion Basin, Phase If 
SAMPLE NAME: FRB-0603

Loeation (SRS Coordinales); 53775 E $76260 \mathrm{~W}$

Sampie Matrix: Soil

Constituent

Melal Paramelers

Arsenic, total secoverable

Bsirium, $100 \mathrm{al}$ recorvable

Beryllinm, tota[ recoverable

Chromitom, rocal recoversble

Njckel, totel recoverble

Lead, 10 al 1 recoversbic

Thatlioun, iotal recoverable

Radlological Paramekers

Gross alpha

Americ[um-24]

Bsกium-133

Noprolatille beta

Carbon-14

Cobalt-60

Cesium-137

Eyropium-154

Iodiat-129

Fotessiug 40

Nickel.63

Pturopitup-239/240

Radium-226

Strontum-90

Tedintion:99

Uranium 238

Semivoletile Paraneters

Dibenzofuran

Diry-butyl phthalate

N-Nitrosodipherylenine

2-Chiorophenol

Valtotile Paramevers

Dichloromethane (Melhylent chloride)

Carbon disoliside

Toluene

Trichloroethylene
RQ AQ B Result

(10: 1072

Interval Depths: t2,00 to t4,00

Percent Soljds 77.00

Unit D.Limit Method

$$
\begin{gathered}
4.55 \\
20.10 \\
0.481 \\
35.40 \\
7.52 \\
14.00 \\
6.84
\end{gathered}
$$

$\begin{array}{ll}\text { mgkg } & 6.84 \\ m g / k g & 3.42 \\ \text { meks } & 3.42 \\ \text { mg/kg } & 3.42 \\ \text { mikg } & 3.42 \\ \text { me/kg } & 6.84 \\ \text { mefks } & 6.84\end{array}$

EPA6010

EPA6010

EPA6010

EPÁ6010

EPA010

EPAGOIO

EPA601O

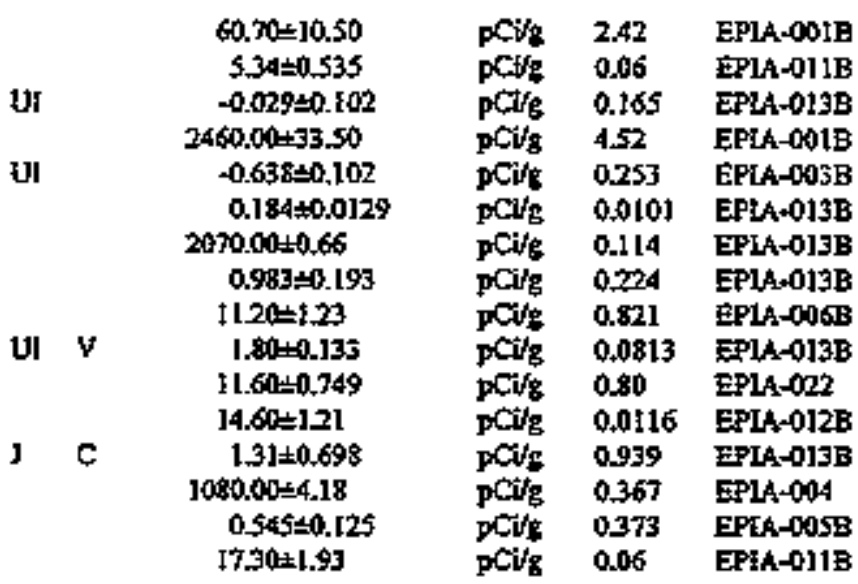

$\begin{array}{lll}u & & 42.60 \\ u & v & 49.90 \\ & & 16.60 \\ & & 42.60\end{array}$

4.RKg 42.60

$46 / k$ s 42.60

HORg $4 \mathbf{2 . 6 0}$

$\mu$ flig 4260

EDPA8270

EPA8270

EPA8Z70

EPA8270

$\begin{array}{llll}\text { U } & \text { vo } & \text { H } & 0.39 \\ \ddagger & 0 & \mathbf{H} & 0.325 \\ \mathbf{U} & \text { vo } & \mathrm{H} & 0.403 \\ \mathbf{v} & \mathbf{O} & \mathrm{H} & 0.0549\end{array}$

$\begin{array}{lll}\mu \mathrm{e} / \mathrm{kg} & 0.649 & \text { EPA\&260 } \\ \mu \mathrm{g} / \mathrm{kg} & 1.30 & \text { EPA8260 } \\ \mu \mathrm{g} / \mathrm{kg} & 0.649 & \text { EPA\&2C0 }\end{array}$

ADkg 0.0649 EPA8260

F-Area Retention Basin, Phisc II 
SAMPLE NAME: FRB-0604

locarion (SRS Coordinates): 53775 E $76260 \mathrm{~N}$

5ample Motrix: Soil

Coostitvent

Metal Parameters

Asțent5, 10lal rectiverable

Bariura, total recoverable

Beryllitan, total recoverable

Chromium, total recoverable

Nickel, rotni recoverable

Lead cotal rexoverible

Thalliurn, otid recoverable

Radiologieal Parmeters

Gross alpha

Amerieflim-24]

Baviun-133

Nompolatile bea

Cabon-14

Cobali -60

Cetivin: 137

Europitum-1S4

Jorine-129

Potessium 40

Nickel.63

Pluknium+239/246

Padiag -226

Sirention -90

Texhnetionos:99

Urarium-238

Semiwolatile Parmeters

Dibeneofurin

Di-n-boryl phchalete

N-Whtrosodiphemylamits

2-Chloraphemal

Volaule Paramelers

Diebloromethane (Methylene chloride)

Carbon disolfide

Toluene

Trichtorocthylene

Sample ID: 102254

Interval Depuhs: 14.0010 I6.00

Pertent Solis:s: 76.00

RQ AQ B Restl Unit D.EInit Mettod

6.93
6.40
0.09
19.40
1.69
11.20
6.93

IDIkB 6.99

mgikg 3.46

$\mathrm{mog} / \mathrm{kg} \quad 3.46$

$\mathrm{mg} / \mathrm{kg} \quad \mathbf{3 . 4 6}$

ग2/ $/ \mathrm{kg} 3.46$

mig/kg 6.93

Ing/kg 6.93

EPÁ́010

EPA6010

EPA6010

EPA6010

EPA6010

EPA6010

EPAGOLIO

U

\begin{tabular}{|c|c|c|c|}
\hline $\begin{array}{l}51.80 \div 10.40 \\
0.0017+0.02 \hat{s}\end{array}$ & $\mathrm{pCH}_{\mathrm{g}}$ & $\begin{array}{l}3.04 \\
0.054\end{array}$ & $\begin{array}{l}\text { EPLA-001B } \\
\text { EPLA-01IB }\end{array}$ \\
\hline$-0.0038 \div 0.0307$ & $p c w_{p}$ & 0.0152 & EPLA-0]3B \\
\hline $270.00=24$ & $\mathrm{pCirg}$ & 4.50 & DOLE \\
\hline$-0.655 \leq 0.101$ & $\mathrm{pCl} / \mathrm{s}$ & 0.255 & EPLA-00BB \\
\hline $0.0005=0.0043$ & $\mathrm{CV}$ & 0.0073 & EPLA-013B \\
\hline $0.966 \pm 0.0164$ & $\mathrm{pCV}$ & 0.0104 & 0138 \\
\hline $0.0144 \pm 0.0428$ & $\mathrm{pCI}$ & 0.0453 & 013B \\
\hline$-0.0542 \div 0.133$ & pC & 0.303 & EPLA-006B \\
\hline $1.85=0.123$ & $\mathrm{pC}$ & 0.0627 & EPLA-0138 \\
\hline $0.961 \div 0.381$ & $i$ & 0.738 & EPLA-O2C \\
\hline $0.186 \pm 0.0557$ & $\mathrm{pCV}$ & 0.06 & EPIA-BI2B \\
\hline $1,07=0.12$ & pCI & 0.093 & EPLA-0E9B \\
\hline $747.00 \pm 3.63$ & $\mathrm{PCH}$ & 0.384 & EPLA-OOA \\
\hline $0.0326 \pm 0.099$ & $\mathbf{n C}$ & 0.316 & EPLA-005B \\
\hline 1.1$\} \pm 0.151$ & $\mathrm{p}$ & 0.06 & EPLA-0:1E \\
\hline
\end{tabular}

y)

บ1

U」

ט]

บี

U]

a] $v$

$\Rightarrow \mathrm{C}$

U.

$1.1\} \pm 0.151$

$\begin{array}{lll}U & & 43.70 \\ U & V & 25.50 \\ U & & 43.70 \\ U & & 43.70\end{array}$

$\mu \mathrm{kg}$

Meikg

D:RKg

$\mu \rho k g$

43.70

43.70

43.70

43.70

EPA8270

EPA 8270

EPA8270

EPA8270

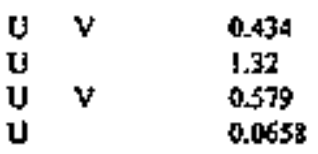

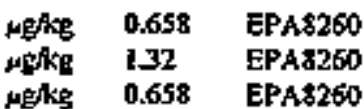

Hoks 0.0658 EPA8260

F-Are Retention Basin, FhaseI 
SAKLLENAME: FRE-0605

Location (SRS Courdinates): 53775 E $76260 \mathrm{~N}$

Sample Marixix Soil
Coustituent

Mejal Paraneters

Arsectic, tatal recowerable

Bajium, total recoverahle

Beryllium, total recoverable

Chromism, zots recowerable

Nikkel, wotol tecoverable

Lead, cotal roweoveratile

Thall|und, tolal recoverable

Rsdiologicot Paramelers

Gross alphs

Amaricium-241

Barmin-133

Nonwolstilt bets

Carbon-14

Cobali- 60

Cesium-137

Europium-154

Iodine-129

Potassium-40

Nicket-53

Plutanitum-239/2419

Raส̛ư-2016

Stronlium-90

Technetium-\$g

Uranime238

Sominotaile Parametors

Dibenzotiunan

DE-n-butyl phthalale

N-Ninosodiphenylartins

2-Chloropletiol

Volatile Paranerer:

Dicbloromethare (Mestiylene chloride)

Carbon diswlidide

Tolvene

Trichlaroerhyicnt

ע

$$
\begin{gathered}
6.89 \\
6.49 \\
0.159 \\
39.10 \\
1.35 \\
15.10
\end{gathered}
$$

J

6.84

$1950+5=7$

U)

$404,00=1390$

J C

UI

$339.00 \div 2.40$

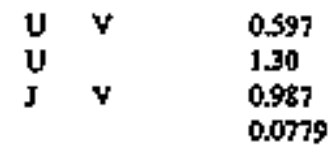

RQ AQ B Resnkt Uati D.Limlt Method

Sample ]D: 102255

Interval Depths: 6.600 to 18.00

Percent Solids: 77.00

$\begin{array}{lll}\text { mg/kg } & 6.34 & \text { EPA6010 } \\ \text { mg/kg } & 3.42 & \text { EPA6010 } \\ \text { mg/kg } & 3.42 & \text { EPA6010 } \\ \text { mg/kg } & 3.42 & \text { EPA6010 } \\ \text { mg/kg } & 3.42 & \text { EPA6010 } \\ \text { mp/kg } & 6.84 & \text { EPA6010 } \\ \text { me/ks } & 6.84 & \text { EPA6010 }\end{array}$

pcis's $\quad 2.96$

EPLA-DOJB

$0.002=0.026 \quad \mathrm{Pi} / \mathrm{g} \quad 0.0612$ EPLA-011B

$0.0064 \div 0.0105$

PCis 0.0136 EPLA-013B

PCi's 4.56 EF[A-0B]B

$\begin{array}{lllll}\text { U: } & -0.685 \div 0.101 & \mathrm{pCi} / \mathrm{g} & 0.233 & \text { EPLA-003B } \\ \text { U: } & 0.0026 \pm 0.004 & \mathrm{pCV} / \mathrm{g} & 0.0067 & \text { EPLA-013B }\end{array}$

$337=0.0285$ PCis 0.0102 EPLA+OH3B

UI $\quad .0 .076 t \pm 0.0275$ PC) $0.05 \$ 1$ EPLA+013B

UI $0.0143 \pm 0.102 \quad \mathrm{pCV} / \mathrm{g} \quad 0209$ EPLA-0068

UI $V \quad 2.16 \pm 0.116 \quad$ PCis 0.0557 EPLA-013E

UI 0.6340 .377 PCV $0.7 \%$ EPLA 027

UI $0.0055 \pm 0.0255$ pCis 0.06 EPA-012B

pCifs 0.06

pCig $\quad 0.337$

$0.0579 \pm 0.0995$

pCis 031

$0.965 \pm 0.201$

PCivg 0.06

EPLA-005B

EPLA-01]B

F-Area Revention Batin, Fhose II

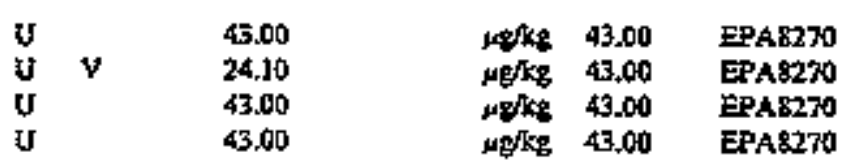

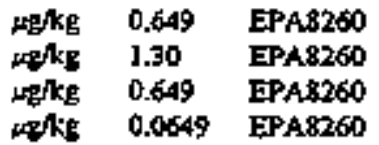


SAMPLE NAME+ FRB-0606

Location (SFS Coordigats): 53775 E $76260 \mathrm{~N}$

Sampit Matrix: Soil

Constlibent

RQ AQ B Result

Sample ID: 102256

Internal Depths: 18.001020 .00

Percept Solids: 83.00

Metal Paranters

Asenic, 10tal recoverable

Barium, total recouverable

Beyllium, total recoverible

Chrominer, total recowtable

Nitiket, total recoverable

Lest, tobj recovarable

Thallium, total recoveratio

Radiological Parameters

Gross alphs

Americium-24)

Barium-133

Nonvolatile bee

Carbogn-14

Cobal:-60

Cosiume-137

Europium-154

Iodine-129

Potassism-40

Nickel-63

Piotonima-23/240

R2Gium-206

Strontiun-90

Techatilnem-99

Uranism-238

Sernivolatio Parandars

Dibenzofuras

Dim-butyl phthalate

N-Nimosodiphenylamine

7-Chlorophenol

Valatile Parameters

Dichloromethane (Methylene chloride)

Carbon disulifide

Taluente

Irichlorocthylese
UI

Ut

UI

UI

UI

Ut

UI V

J

ปI

$\begin{array}{cc}0 & 6.34 \\ & 4.22 \\ & 6.0913 \\ & 26.60 \\ & 0.635 \\ & 8.86 \\ U & 6.34\end{array}$

$5.39 \div 2.67$

$0.0273 \div 0.0249$

$-0.147 \pm 0.103$

$21200 \div 9.92$

$-0.598 \div 0.205$

$0,0038=0.004 \$$

$22.00 \div 3.20$

$-0.0019 \pm 0.0271$

$0.0325=0.097$

$1.90 \pm 0.223$

5. $10=0.435$

0.147士4.05I I

$0.977 \pm 0.0985$

$360,00 \pm 2,24$

$0.118 \pm 0.102$

$\$ .34 \pm 0264$

pCive

2.27

pCis 0.06

pCt/ 0.0224

$\mathrm{PCV} \quad \mathbf{3 2}$

$000 / 4 \quad 0.0072$

pCis 0.0186

$\mathrm{pC} / \mathrm{s} \quad 0.0399$

pCing 0.187

PCis 0.0678

$\mathrm{pCH} / \mathrm{s} \quad 0.84$

pCof 0.0126

pCl/s $\quad 0.0759$

PCU/s $\quad 0.293$

pots 0.062

EPLA-00IB

EPIA-03IB

EPIA-Ot3B

EP1A-0018

EPIA-003B

EP1A-015B

EPLA-Ot3B

EPIA $013 B$

EPLA-0068

FPIA-OI38

EPLA-100?

EPIA-012B

EPIA-O13日

EPIA-OOS

EP1A-005B

닥ㅅ-이요

$\begin{array}{lll}u & & 39.40 \\ U & v & 18.50 \\ u & & 39.40 \\ u & & 39.40\end{array}$

woks 39,40

wefkg 39.40

मrisc 39,40

toks 39,40

EPA8270

EPA8270

EPA:270

EPA8270

$\begin{array}{lll}u & v & 0.373 \\ \mathrm{u} & & \\ \mathrm{u} & \mathrm{v} & 1.20 \\ & & 0.446 \\ & & 0.0602\end{array}$

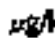

wolke 120

$-0, \mathrm{~kg} \quad 0.602$

a k
0.0602

EPA8Z60

EPA8260

ㅌPA8260

EPA\$ 200
PCis 0237

$\mathrm{pCd}$ 0.235

F-Arca Retcplion Basin, Phase ?I 
SAMPLENAME+ FRB-0701

Lomtion (SRS Coordinates): 53732 E $76132 \mathrm{~N}$

Sample Matrix: Sotl

Conslituent

Metal Parametes

Arsenic, tolat recoverabte

Brrium, total nocoverable

Bayflium, tobl recorverable

Chourius, iotol teroverable

Nitekel, total recowerable

Lead, total roeoverable

Thallinn, total recoverable

Radiological Parancters

Gross alpha

Americhum-241

Bsinumat133

Nonvolatik bets

Carbon-14

Cobalk-60

Cesium-137

Europium-154

lodiut-129

Potassitum-40

Nickel-63

Fitutonitum-239/240

Radiurn-206

Soontium-90

Technelism-99

Uranium-238

Stmivolatile Pararaters

Diberzonfuran

Di-n-bueyl phulatate

N-Nitroesdphenylamiose

2.Chlorophenol

Volatile Parameler:

Dichloromelyant (Mebylene chloride)

Caron disulfite

Toluenes

Trutbioroethylene

บ
RQ AQ $\mathbf{B}$ Result Unit D.Limit Methed

Irtern: Depthe 0.00 to 1.00

Percent Solids. 90.00

\begin{tabular}{|c|c|c|c|c|}
\hline \multirow[b]{2}{*}{$v$} & 5.85 & migks & 5.85 & EPA6010 \\
\hline & 20.10 & - mplog & 292 & EPA6010 \\
\hline & 0.185 & molkg & 2.92 & EPA60 \\
\hline & 2.17 & mog/k8 & 292 & EPA60 \\
\hline & $2.0 t$ & $\mathrm{mglxg}$ & 292 & EPACOO \\
\hline & 5.15 & mglkg & 5.85 & EPAGD \\
\hline & 5.85 & melks & 5.85 & EPA60 \\
\hline
\end{tabular}

\begin{tabular}{|c|c|c|c|c|}
\hline$x$ & $4.39 \leq 224$ & $\mathrm{pC} / \mathrm{s}$ & 2.56 & EPLA-00tB \\
\hline$x$ & $0.007 \pm 0.0183$ & pCiss & 0.06 & EPLA-0) lD \\
\hline$x$ & $0.0004=0.0071$ & $\mathrm{pCV}$ & 0.0106 & EPLA-013B \\
\hline$x$ & $3.77 \pm 3.18$ & $\mathrm{CO} / \mathrm{g}$ & 6.56 & EPLA-00IB \\
\hline$x$ & $0.0157=0.0555$ & $\mathrm{pCOH}$ & 0.163 & EPLA-005B \\
\hline Iاט & $-0.0012 \pm 0.0053$ & $\mathrm{PC} / \mathrm{s}$ & 0.0079 & EPLA-013B \\
\hline$x$ & $0.166 \pm 0.0143$ & pcis & 0.0099 & EPHA-0I3B \\
\hline$x$ & $+0.0094=0.053[$ & $\mathrm{PCI} / \mathrm{g}$ & 0.0761 & EPLA-013B \\
\hline \multirow[t]{2}{*}{ UIנ } & $-0.0433 \pm 0.047$ & peis & 0.09 & EPLA-006B \\
\hline & $1.74 \div 0.155$ & $\mathrm{pCH} / \mathrm{g}$ & 0.0867 & EPLA-OFBB \\
\hline$v$ & $0.849=0.34$ & $\mathrm{OCH} / \mathrm{s}$ & 0.659 & EPLA.OZ \\
\hline U & $0.0033 \pm 0.0136$ & $\mathrm{pCl} / \mathrm{g}$ & 0.06 & BPLAIDB \\
\hline $\mathbf{x}$ & $0.931 \leqslant 0.0712$ & $\mathrm{pCls}$ & $0.0 \mathrm{~d}\} \mathrm{d}$ & EPLA-0138 \\
\hline $\mathbf{v}$ & $1.75 \pm 0.443$ & $\mathrm{pCl} / \mathrm{g}$ & 0.434 & EPLA-004 \\
\hline บี & $0.0952=0.168$ & $\mathrm{pCd} / \mathrm{g}$ & 0288 & EPLA-OOSB \\
\hline$x$ & $0.919=0.155$ & $\mathrm{pCt} / \mathrm{g}$ & 0.06 & EPIA-OI 1B \\
\hline
\end{tabular}

$\begin{array}{llll}\text { UJ } & \text { IY } & \mathbf{P} & 36.00 \\ \text { UJ } & \mathbf{T Y} & \mathbf{P} & 36.00 \\ \text { UJ } & \Gamma Y & \mathbf{P} & 36.00 \\ \text { UJ } & \mathbf{I} & \mathbf{P} & \mathbf{3 6 . 0 0}\end{array}$

山U: $36.00 \quad$ EPA8270

$\mu \mathrm{g} / \mathrm{kg} 36,100$ EPABZ70

$4 \mathrm{~B} / \mathrm{kg}$ 36.00 EPAB270

MERIS 36.40 EPAS270

\begin{tabular}{|c|c|c|}
\hline Ut & gJYO P & 0.556 \\
\hline ए] & IYO P & I.£) \\
\hline UJ & VBF" $P$ & 0.t1] \\
\hline Uf & IYO $P$ & 0.0556 \\
\hline
\end{tabular}

4P/kg 0.556 EPA8260

HEkg 1.1] EPA 260

मolkg 0.556 EPA3260

$\omega \mathrm{g} / \mathrm{kg} \quad 0.0556$ EPA8360

$\cdot=\mathrm{OB}$

F-Area Reteption Basin, Phase il 
SAMPLENAME: FRB-0762

Losation (SRS Coardinates): 53732 E $76132 \mathrm{~N}$

Sample Marrix: Söl

Constifuent

Metsl Paramerers

Asenic, total reconerablo

Bufilm, 100al recoverabl.

Beryilifur, totol recansabje

Chromìn, 10 and recoverable

Nickel, total recooverable

Lad 1ocal recoverste

Toallium, toral recowerable

Ratiological Parameters

Gross atphs

Americtum-24]

Bariun-133

Norivolatile beb

Carbor-[4

Cobai $[-60$

Cesiur-137

Europium-154

Lodine-129

Potassiun-40

Nisket-693

P1otoníam-279240

Radfan-226

Strontiven-90

Technetiono-99

Ur:ainom-238

Serriwolatile Parameters

Dibenzotural:

Di-n-buryl pathajare

N-Nitrosodiphenylamine

2-Chloropheral

Volatile Parameters

Dtebloromeuhase (Methylene chloride)

Carbon distulfide

Toluene

Trichlorofityylare
RQ AQ B Result

U

5.91
24.10
0.256
10.10
2.06
8.27
5.91

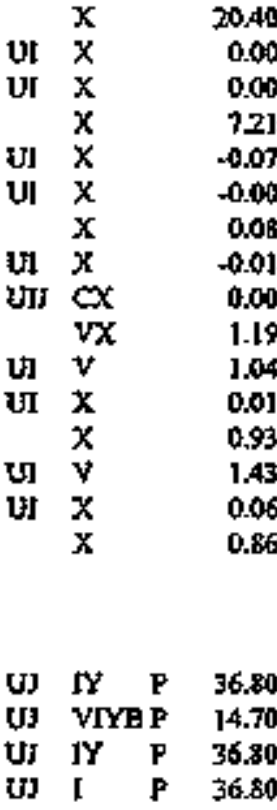

v

8.27

$0.0045 \div 0.0207$

$0.0037=0.007$

12]표.57

$14 \pm 0.0045$

$0.0884=0.0125$

$0.0188=0.0478$

$0.001]=0.044$

1. 1 올ㅇ.134

J.04:0.388

$0.0118 \pm 0.0142$

$0.93 \pm 0.0546$

$1.43 \div 0.342$

$0.0695 \pm 0.173$

$0.869 \div 0.147$
Saraple 1D: 102291

Interval Depths: 2.00 50 400

Pereent Solids $\$ 9.00$
Unit D.litinit Mebiad

mikg 5,91

mgikg 296

$\mathrm{mg} / \mathrm{kg} \quad 296$

mefs 296

$\mathrm{m} / \mathrm{kg} \quad 2.96$

mejks 5.91

midnts 5.91

EPA6010

EPA6010

EPA6010

EPA6010

EPA6에임

EPAG010

EPA6010

\begin{tabular}{|c|c|c|}
\hline $\begin{array}{l}\mathrm{CN} / \mathrm{s} \\
\mathrm{CH} / \mathrm{s} \\
\mathrm{CT} / \mathrm{s}\end{array}$ & $\begin{array}{l}6.29 \\
0.06 \\
0.0115\end{array}$ & $\begin{array}{l}\text { EPIA-001B } \\
\text { EPLA-011B } \\
\text { EPLA-BIJB }\end{array}$ \\
\hline Fitg & 6.71 & 60IB \\
\hline $\mathrm{Cit}$ & 0.163 & EPI \\
\hline $\mathrm{Ct}$ & 0.0078 & EPIA \\
\hline $\mathrm{Cif}$ & 0.0096 & EPt \\
\hline $\mathrm{CI}$ & 0.0 & EPPI \\
\hline $\mathrm{Ci}$ & 0,0888 & EPL \\
\hline Ci & 0.0763 & EPI \\
\hline C: & 0.746 & EPI \\
\hline & $0.0 \mathrm{~s}$ & EP1 \\
\hline 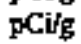 & 0.0394 & at $3 B$ \\
\hline $\mathrm{Ci}$ & (1) 332 & EPIA-OOA \\
\hline $\mathrm{pct}$ & 0.731 & EPLA OOSB \\
\hline & 0.0663 & 0.18 \\
\hline
\end{tabular}

10/6 $36 \%$

EPA2270

एPAS270

$\mu g / k \mathrm{k} \quad 36.80$

mikg 36,80

EPA8270

mojkg 36.80

SPA8270

F-Area Relemion Ersin, Phase 13 
SAMPLE NAME: FRB-0703

Location (SRS Coordinalos): $\$ 3732$ E $76137 \mathrm{~N}$

Somple Matrix: Soll

Copstituent

Metal Paranerets

Assenic, total rocoveratblo

Bariun, totsi recoverable

Brylinuth, wolal recoverable

Chromitam, 10tal recowerable

Nipkel, woll recoverable

Lead, e이 recowerabte

Thrallium, total reconcrab|t

Radiglogicat Parameters

Gross ajphs

Ambricitim-241

Baritsm-133

Nomvolatile beta

Cromon-14

Cobalk-60

Cesium +137

Europiurn-154

Lodiue-129

Potassiun-40

Nickel-63

Plsionium-239/240

Radium?226

Stronlium-90

Techunetilum-99

Uraniom-238

Semivolatife Parmelers

Diberneofwan

Dj-a+buxyl phthalate

N.Niturosotphemylamine

2-Chlorophesol

UJ IY $P \quad 37.50$

UJ VTB $P$ 2030

Uf IY P 3750

UJ IV $P \quad 3750$

Volaule Paraneiers

Dibloromethane (Medhylene chloride)

Cabon disolfide

Tolnete

Triehloroethylene

$\begin{array}{llll}\text { UI } & \text { BIY } & \text { P } & 0,568 \\ \text { UJ } & \text { JY } & \text { P } & 1.14 \\ \text { UJ } & \text { V8CB } & \text { P } & 0.125 \\ \text { UJ } & \text { IY } & \text { P } & 0.0568\end{array}$

RQ AQ B Result

Unit

D.Linglit Method

U

UI $x$

Ul $x$

$02=6,96$

$-0.0139+0.0184$

$0.0117=0.0173$

$247000=1290$

$-0.0867 \div 0.0815$

0.0230 .0103

$6.60 \div 0.07$

$-0233-0.0617$

$0.659 \div 0.328$

1.47 40.16

$\begin{array}{lll}\text { UI } & V & t .66 \pm 0.422 \\ \text { uI } & X & 0.0495 \pm 0.025)\end{array}$

$0.941 \div 0.066$

$91.30 \div 1.19$

$0.223 \pm 0.115$

$10.51+1.04$

PCis 3.5

acio 0.0213

pCifs 8.03

JCis 0.165

pCis 0.0096

pcils 0.0155

pCila 0.0\$2

pCiss 0.782

pCis $\quad 0.0823$

pCty $\quad 0.76\}$

$\mathrm{pClig} \quad 0.06$

$\mathrm{PC} / \mathrm{s} \quad 0.0538$

$\mathrm{pCi} / \mathrm{g} \quad 0265$

pCis 0.308

0.06

EPAGDIO

EPA6010

EPAEOIO

EPA6010

EPA6010

EPAGOIO

EPAGDIO
EPlA-001B

EPLA-0SIB

EP]A-013B

EPLA-OOIB

EPLA-005B

EPLA+이3B

EPLAALISB

EP1A-013B

EPLA-006B

EPIA-013B

EPUA-022

EFIA-012E

EPLA+013B

SPIA-0.04

EPLA-O0SB

EPIA-OIIB

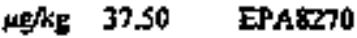

$\mu \boxminus k E$ E 37,50 EPA8270

HЮK 3750 EPA8270

\#ל/ks 37,50 EPAs270

$4 \mathrm{~g} / \mathrm{se}$

0.568

EPA8260

Nofk I.14 EPA8760

polkg 0.568 EPA8260

MeNk 0.0563 EPA8260

F-krea Retention Batin, Phose II 
SAMPLE NAMAE: FRE-0704

Location (SRS Coodinnes): 53732 E $76132 \mathrm{~N}$

Sampit Malrix: Soil

Coustifuent

Mecal Pararaelers

Arsenic, iotal recoverable

Barium, total recoverable

Berytsin, total recorerable

Chromfum, rotal recowerable

Nickel, total recoverable

Lead, toal necoverbile

Thallimn, tolal recowterabie

Radialogical Parameters

Gross alpha

Americiump-241

Barมm-133

Nonwolarile beta

Carbon-14

Cotait 60

Cesium-137

Evropiun-154

Jodine-129

Polassiund 40

NIJkel-63

Ptolopion-239/240

Radium-226

Strontiom-90

Tecknetrum-99

Uripinat1-238

Sernivoletile Parametcrs

Diberzofurar

Di-a-buryl pothalae

N-Nitrasodipherylamine

2-Chloropherol

Volalile Parameters

Dichloromethent (Methylent chlonde)

Cabon distifrde

Tolvere

Trieblatoelhylene
$\mathbf{R Q} \mathbf{A Q}$ Ferult

Sample ID: 102294

Interval Depils: 10.00 vo 12.00

Purcent Solids: 87.00

Unit D.LAmit Method

บ

3.26
8.38
0.148
12.70
2.43
6.32
6.05

$\begin{array}{lll}\text { mg/kE } & 6.05 & \text { EPA6010 } \\ \text { mEdkg } & 3.02 & \text { EPA6010 } \\ \text { mykg } & 3.02 & \text { EPA6010 } \\ \text { mg/kg } & 3.02 & \text { EPA6010 } \\ \text { mg/kg } & 3.02 & \text { EPA6010 } \\ \text { mekg } & 6.05 & \text { EPA6010 } \\ \text { mgikg } & 6.05 & \text { EPA6010 }\end{array}$

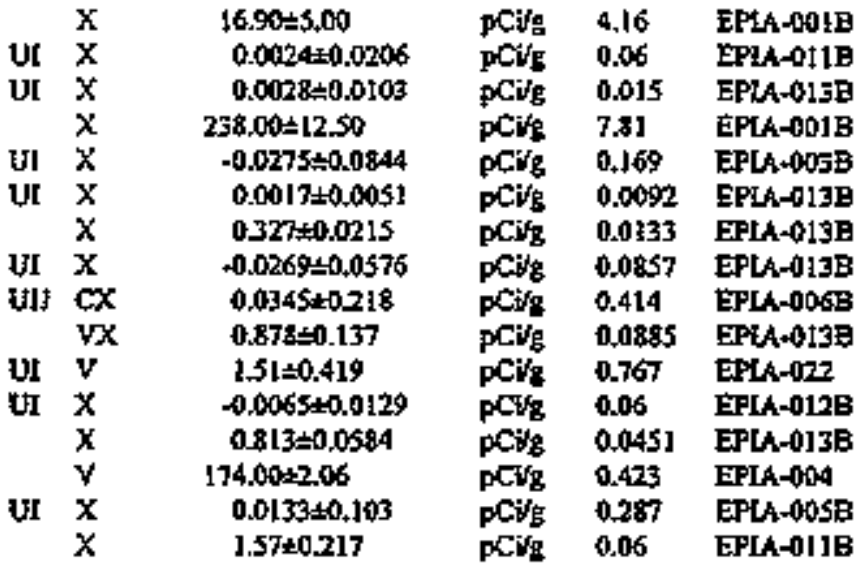

UJ if $P \quad 38.20$

U] VIYE P 15.30

U3 IY $\$ \quad 3820$

GJ I $P \quad 38.20$

Hgkg 38.20

EPA8270

$\mu g / \mathrm{s} \quad 3820$

$\mu$ efg 38.20

EPA 8270

EPA 270

rokg 3820

EPA\$270

F-Area Retention Bäion, Phase II

\begin{tabular}{|c|c|c|}
\hline J & $81 Y \mathrm{Y}=$ & 0,379 \\
\hline U: & IY & 1.15 \\
\hline UJ & VSI" $P$ & 0.126 \\
\hline UJ & IY & 0.0575 \\
\hline
\end{tabular}

$\begin{array}{lll}\mu E / k g & 0.575 & \text { EPAB260 } \\ \mu / \mathrm{kg} & 1.15 & \text { EPAB260 } \\ \mu \mathrm{B} / \mathrm{kg} & 0.575 & \text { EPA8260 } \\ \mu \mathrm{g} / \mathrm{kg} & 0.0575 & \text { EPA8260 }\end{array}$ 
SAMPLE NAME: FRB-070S

Location (SRS Coocdinales): 53732 E 76।32 N

Sarmple Matrix: Solt
Assuric, tolel recoverable

Banim, tosl recowerable

Beryitim, notat recoverafole

Chrominim, 10tal recoverable

Nickel, total retoterialis

Lead total teconsable

Tálium, conl recoverable

Radiological Paramerers

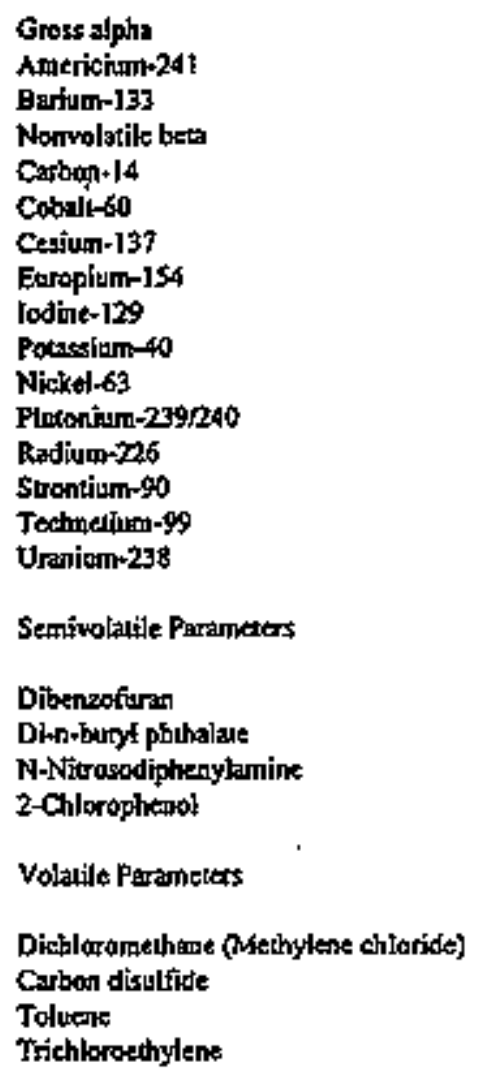

RQ AQ B Result

U

Sample ID: 102295

ianerval Depths: 12.00 to 14.00

Percent Solids: 89,00

Unts D.Limit Methad

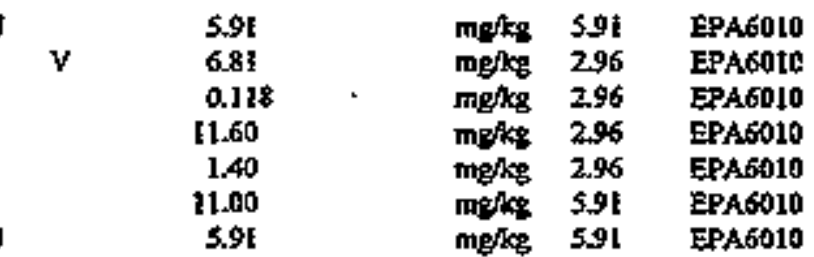

\begin{tabular}{|c|c|c|c|c|}
\hline$x$ & $24.00=4.14$ & $\mathrm{pct} / \mathrm{s}$ & 3.92 & EPIA-0OIB \\
\hline$x$ & $-0.008=0.0204$ & PCifg & 0.0615 & 5PEA-011 \\
\hline$x$ & $0.0005=0.0037$ & pCils & 0.00114 & EPLA+01: \\
\hline $\mathbf{x}$ & $27.60 \leq 4.88$ & $\mathrm{PC} / \mathrm{s}$ & 6.54 & $E P \mid A=0$ \\
\hline $\mathbf{x}$ & $-0.0254 \leq 0.0834$ & pcigg & 0.163 & EPLA-00 \\
\hline$x$ & $+0.0033 \pm 0.0044$ & $\mathrm{pCis}$ & 0.0075 & EPLA-013 \\
\hline$x$ & $0.071=0.0119$ & $\mathrm{pCl} / \mathrm{g}$ & 0.0109 & EPLA-012 \\
\hline$x$ & $-0,0201=0,0432$ & $\mathrm{pCls}$ & 0,0705 & ㄷP $[A-01:$ \\
\hline$C x$ & 0.001300 .116 & $\mathrm{pC} / \mathrm{s}$ & 0.246 & -006 \\
\hline$v x$ & 0,714 告0,121 & $\mathrm{pCols}$ & 0.0765 & EPLA-013 \\
\hline$\checkmark$ & $1.59=0.524$ & $\mathrm{pCt} / \mathrm{s}$ & 0.995 & ISPLA-02 \\
\hline$x$ & $-0.0(9)=0.0238$ & pCls & 0,0662 & EPLA-01 \\
\hline $\mathrm{X}$ & 0.63240 .0695 & $\mathrm{pCi} / \mathrm{g}$ & 0.0519 & E्PF $A-013$ \\
\hline $\mathbf{v}$ & $23.40 \pm 0.672$ & $\mathrm{PC} / \mathrm{s}$ & 0,369 & EPLA-OBA \\
\hline$x$ & $0.0719 \pm 0.0995$ & $\mathrm{PCS} / \mathrm{g}$ & 0269 & EPIA-00S \\
\hline $\mathbf{x}$ & $1.05 \pm 0.367$ & $\mathrm{pet} / \mathrm{s}$ & 0.06 & $E P 1 A-011$ \\
\hline
\end{tabular}

$\mu g / k$ 37,30 EPA8270

$\mu g / k g$ EPA8270

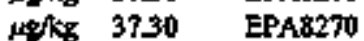

Hylke 37.30 EPA8270

\begin{tabular}{|c|c|c|c|c|c|c|}
\hline U3 & $\mathbf{s i} Y$ & $\mathbf{F}$ & 0.562 & $\mu$ 伤 & 0.562 & EPA8260 \\
\hline U & JY & $\mathbf{P}$ & 1.12 & $\mu \mathrm{g} / \mathrm{kg}$ & I.12 & EPA8260 \\
\hline UJ & V8]* & $\mathbf{P}$ & 0.124 & 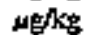 & 0,562 & EPA 8260 \\
\hline UJ & JY & $\mathbf{P}$ & 0.0562 & $\mu g / \mathrm{kg}$ & 0.0562 & EPA 860 \\
\hline
\end{tabular}

F*Area Retertion Basion, Phase !1 
SAMPLE NAMUE: FRE-0706

Lecation (SRS Coordinates): 53732 E $76132 \mathrm{~N}$

Smple Matuix Soil

Constituent

Mectal Parameters

Arstaic, tasal recoverable

Barium, total Jecotrersbite

Beryllium, total recoweroble

Chrowium, total recoverable

Nickel, copil restrvesble

Lead, tobl recoveroble

Thallina, rotai recoverabie

Radjological Parameters

Grots alpha

Ameritium-24]

Barhm-133

Jlonvalatik beta

Cabor-14

Cotbal-60

Cesium-137

Europinn-154

lodine-179

Porassingen-40

Nickel-6s

P(utonhum-239/240

Radram-206

Surotitimer-90

Technetiun-99

Uranium-238

Semiwolatile Perameters

Dibenzoforan

Dir-n-bury] phthalate

N-Nìrosodiphenylamine

2-Chlomoghetol]

Volarile Paracoeters

Dicklokomethane (Methylene thlorids)

Carbon disulade

Toluene

Triahlorcetbylene
RQ AQ $\mathbf{E}$ Result

Sample ED: 102796

Interval Depths: 14.00 id 16.00

Pexcent Solits: 89.00

Unit D.Limais Method

I

5.91
6.50
0.11
12.40
1.25
5.84
5.91

ul $x$

$\mathrm{x}$

$21.60=5.32$

ปI $x$

$0,0137 \pm 0,0226$

$0.0059=0.0113$

$25.80 \pm 4.86$

घ] $x$

$-0.0171=0.0853$

$0.01028 \pm 0.0052$

$0.0659 \div 0.0543$

$x$

1] $x$

UIJ $c x$

$-0.0151 \pm 0.0484$

$0.0023 \pm 0.135$

$0.74 \pm 0.138$

$0.99 \div 0.368$

ป1) $v$

$v$

ID $x$

$0.0336 \pm 0.0199$

$0.858 \div 0.0666$

$8.70 \pm 0.607$

$0.0039=0.0989$

0.8 은.15

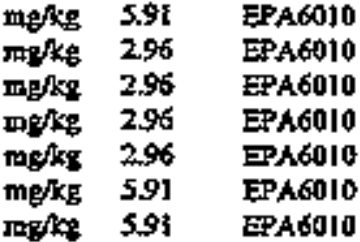

PCis $\quad 5.30$

PCig 0.06

pCW 0.0118

$\mathrm{pCi} / \mathrm{s} \quad 676$

PCi/g 0.166

PCifs 0.0095

$\mathrm{PC}^{\mathrm{N}} \mathrm{g} \quad \mathbf{0 . 0 1 2}$

pCys 0.0798

pCig 0.26

pCis

PCigs

$\mathrm{PCH}$

PCIg

PCis

pCis

pCis

0.0645

0.707

0.06

0.0438

0.437

0.27

0.06

EPLA-001B

EPLA-0J 18

EPLA-0I3B

EPIA-0OIB

EPIA-003B

EPIA-0ISE

EPLA-Q13B

EPLA-013B

EPIA-0065

EPLA-013B

EPLA-022

EPLA.0128

EPIA-013B

EPLA-004

EPLA-ODSB

EPIA-0IL

$\begin{array}{llrl}\text { UJ } & \text { IY } & P & 37.40 \\ \text { UJ } & \text { VYY } & P & 20.20 \\ \text { UJ } & \Gamma & P & 37.40 \\ \text { UJ } & \ddagger & P & 37.40\end{array}$

\section{J}

U) IY $F$

U) V\&LB $P$

U $T P$
0.371

1.12

0.124

0.0562

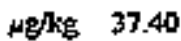

$r$ kg 37,40

rops $\quad 37.40$

소의 37.40

EPA8270

EPAD270

EPA8270

EPA82TO

F-Area Rerention Basin, Phase II 
SAMPLENAME: FRB-0707

LoxBtion (SRS Coocdiartet): 53733 E 76132 N

Sample Matrix: Soil

Constitutpt

Metal Parameters

Arsenic, total recowerabite

Bartura, 10 al recoverable

Berylliun, vota! racoverablo

Chrouajurt, total recoverable

Niekel, tolal recoverstite

Lead, Lotal recoverable

Thalliom, cotal retoverable

Radtological Parneters

Gross alpha

Aupribnim-241

Barmயn-333

Nonvalarile beta

Caton-14

Cobati-60

Cesium-137

Europisom-154

Iodine-129

Polassiam-10

Nickel-63

Plutontum-239/240

kadiun-226

Srontium-90

Techaotiuman-99

Uraniram-238

Serrivotatile Parameters

Disenzofaran

Di.n-butyl phthalare

N-Nitrosodiphenylanine

2-Chlorophenot

Votalite Parameters

Dichloromethane (Mdelhylene chtoride)

Carbon disulfide

Toluepe

Trichlorocthykme

RQ AQ B Result

Sarople ID: 10239:

Interval Dopths: 16.00 to 18.00

Pereent Solids: 89.00

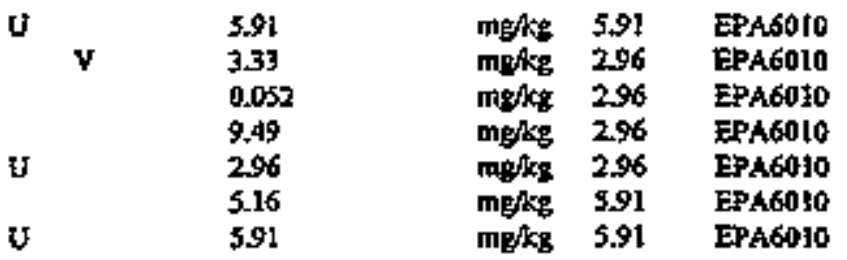

\begin{tabular}{|c|c|c|c|c|c|}
\hline & $x$ & $10.70 \pm 3.55$ & $\mathrm{pCD}$ & 3,54 & EPLA-001E \\
\hline U1 & $x$ & $0.0044=0.00 \$ 5 t$ & $\mathrm{per} / \mathrm{s}$ & 0.06 & ㅎPㄴ서이 \\
\hline \multirow[t]{2}{*}{ U] } & $\mathbf{x}$ & $0.0058 \div 0.0109$ & $\mathrm{pCS}$ & 0.0125 & EPLA-0I: \\
\hline & $x$ & $8.20 \div 3.51$ & $\mathrm{pCi} / \mathrm{s}$ & 6,50 & -00 \\
\hline U] & $\mathrm{x}$ & $-0.0245 \pm 0.0844$ & {$[\mathrm{CH} / \mathrm{B}$} & 0.165 & PLA- \\
\hline \multirow[t]{2}{*}{ U! } & $\mathrm{x}$ & $-0.0006=0.0053$ & pCits & 0,009 & $E P L A-01$ \\
\hline & $\mathbf{x}$ & $0.0963 \pm 0.0141$ & $\mathrm{pCH} / \mathrm{g}$ & 0.0116 & EPlA-OI \\
\hline एा & $x$ & $0.026 \leq 0.0526$ & $\mathrm{pC} \mathrm{s}_{\mathrm{g}}$ & 0.0835 & EPLA-0: \\
\hline \multirow[t]{2}{*}{ UJJ } & $\mathrm{CX}$ & $-0.0393=0.100$ & $\mathrm{pCs} 2$ & 072 & EPLA-O0 \\
\hline & $v X$ & $0.797 \pm 0.15 t$ & pCits & 0.075 & EPLA-01 \\
\hline U] & $\mathbf{v}$ & $1.01 \pm 0.774$ & $\mathrm{pCN}$ & 0.717 & EPLA-0Z \\
\hline \multirow[t]{2}{*}{ ย } & $\mathbf{x}$ & $0.0002<0.0119$ & $\mathrm{pCN} / \mathrm{g}$ & 0.06 & EPLA-01 \\
\hline & $\mathbf{x}$ & $0.914 \pm 0.0618$ & $\mathrm{pots}$ & 0.0453 & EPHA-01? \\
\hline & $Y$ & I. $62=0.341$ & $\mathrm{PC} / \mathrm{s}$ & 0.326 & 포서-00 \\
\hline \multirow[t]{2}{*}{ t] } & $x$ & $0.153 \pm 0.109$ & $\mathrm{pCS}$ & 0.292 & EPIA-O0 \\
\hline & 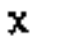 & $0.946=0.16$ & $\mathrm{pC}$ & 0.06 & $A-01$ \\
\hline
\end{tabular}

$\begin{array}{llrl}\text { UJ } & \text { JY } & \text { P } & 36.70 \\ \text { UJ } & \text { VIVB } & \text { P } & 17.20 \\ \text { UJ } & \text { JY } & \text { P } & 36.70 \\ \text { UJ } & \text { J } & \text { P } & 36.70\end{array}$

J St7' P 0.36

US IY $P$ L 1.12

U) $\mathrm{V} 8 \mathrm{CB} P \quad 0.101$

$\begin{array}{llll}\text { Uf } & \mathrm{JY} & \mathrm{P} & 0.0562\end{array}$

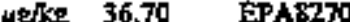

mokg 36.70 EPA\&270

H/kg 36.70 EPA8270

HOAkg 36.70 EPA8270

F-Area Reterston Basin, Phase Il 
This Page Intentionally Left Blank 
SAMPLE NAME: FRB-0801

Localion (SRS Condinates); 53670 E $76156 \mathrm{~N}$

Sample Marix: Sol

Constiluçat

Metal Parantelters

Arsente, rout tecoversole

Barium, rotal recorecable

Eeyllium, toial recoverable

Chromitm, total netorverable

Nickel, folal recoverabie

Losd, solal recovarable

Thablum, total recoverable

Radiologicted Parametts

Gross alphit

Americium-241

Esriom-133

Nonvolatile bets

Carbon+ld4

Cobatit 60

Cesium-137

Emaplam-154

lodine-129

Polassiom-40

Nickel- 63

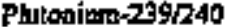

Radinam-226

Strontism-90

Tetupetium -99

Urnium-238

Semivolatüte Paramelers

Dibenzofurm

Di-r-burit pillibatale

N-Nhtosodiphenylaraine

2-Chlorophent

Volatile Parameters

Dichtoromeinzat (Mathylene chlaride)

Carton tosulfide

Toluene

Trichlorvedhyitene
RQ AQ B Eesslt Unit D.Limit Method

Sample 1D: 102282

Intervat Depthr 0.00 to 1.00

FercentSolids. 91.00

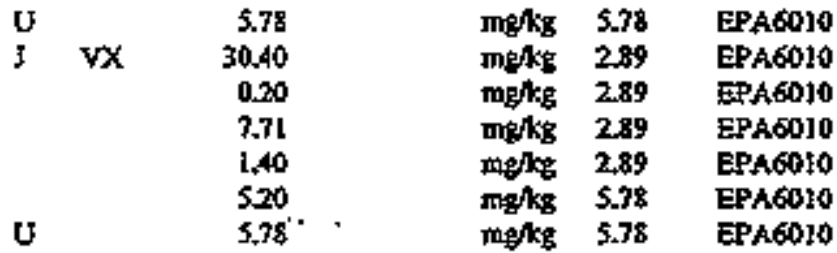

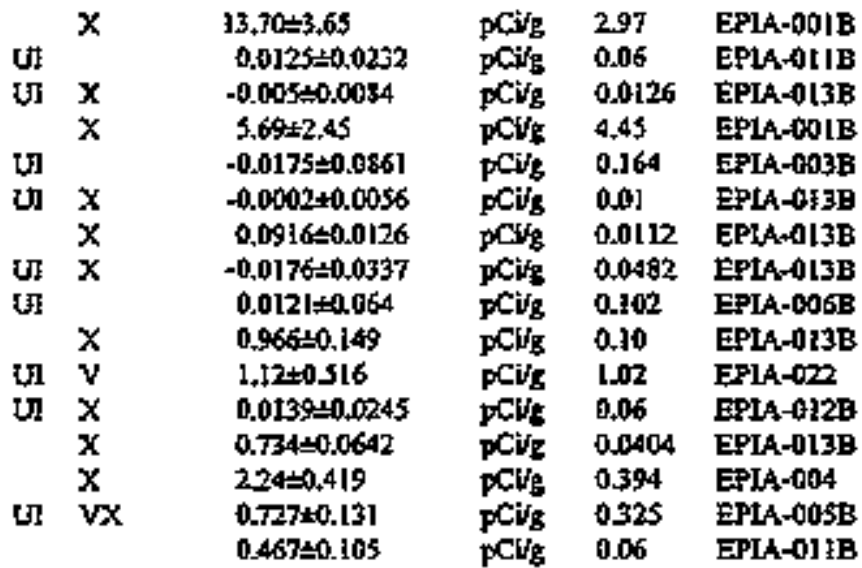

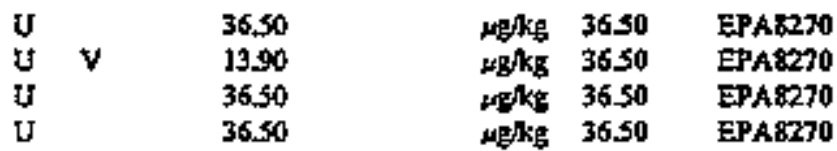

F-Area Retention Basin, Ptrase ]

\begin{tabular}{|c|c|c|c|c|}
\hline $\begin{array}{l}\mathbf{U} \\
\mathbf{y}\end{array}$ & $\begin{array}{l}0.349 \\
1.10\end{array}$ & $\mu g / k g$ & $\begin{array}{l}0.549 \\
1.10\end{array}$ & $\begin{array}{l}\text { EPA8360 } \\
\text { EPA8260 }\end{array}$ \\
\hline $\begin{array}{ll}\mathbf{U} & \mathrm{V} \\
\mathbf{U} & \end{array}$ & $\begin{array}{l}0.187 \\
0.0549\end{array}$ & n=기 & $\begin{array}{l}0.549 \\
0.0549\end{array}$ & $\begin{array}{l}\text { EPA8260 } \\
\text { EPAS260 }\end{array}$ \\
\hline
\end{tabular}


SAMFLE NAME: FRB-080?

Locstion (SRS Conediantes): 53670 E 76156 N

Seraple Marix: Sot
RQ AQ H Retik

$\mathfrak{y}$

Assentic 10tal necorerable

Barin, tolal secowerable

Beryllinm, 10tat rocowerabile

Chromitum, toul recoverate

Nidket, wall Jocoryerable

Lead, totsl recoretrable

Thallium, rotaf recoverable

Radiologiea! Parmeters

Gross aipha

Amperfelum-241

Barien-133

Nonwolarile ben

Contorp-14

Cobsili-60

Cesfure-135

Europitum-154

Iodthe-12s

Poenssium-40

Niekel-63

Pitronium-279R40

Rिक्षेแ⿰⿺⿻一𠃋十-226

\$rostium-90

Techotivum-99

Uranturn-238

Senivalatile Parameters

Dibercofuran

Din-bugil phulalale

N-Nitresodiphenytamine

2-Cblorophenol

Volstbe Parameters

Dichloromechane (Methyierse chloride)

Coton disulfide

Tohene

Trichloroethylene

$\begin{array}{ccc} & & 5.95 \\ & 20.00 \\ & 0.185 \\ & 9.41 \\ & & 215 \\ & & 6.98 \\ & & 5.91\end{array}$

$\begin{array}{rrr}\dot{y} & & \\ \mathrm{u} & & 2250 \\ & v & 44.90 \\ \mathrm{j} & & \\ & & 2.09\end{array}$

Sample ID: 102283

Inucral Depths: 2.00 20 4,00

Percend Solide $\$ 9,00$

Unit D.LImic Meaiod

$\begin{array}{lll}m g / k g & 5.97 & \text { EPA6010 } \\ m g / k g & 2.96 & \text { EPA6010 } \\ m g / k g & 2.96 & \text { EPA6010 } \\ m g / k g & 2.95 & \text { EPA6010 } \\ m / k g & 296 & \text { EPA6010 } \\ m g / k g & 5.91 & \text { EPA6010 } \\ m g k g & 5.91 & \text { EPA6010 }\end{array}$

\begin{tabular}{|c|c|c|c|c|}
\hline $\mathrm{X}$ & $11.60=3.27$ & $\mathrm{xCi}_{\mathrm{g}}$ & 254 & $E P: A=0,1 E$ \\
\hline Et & $0.0118=0.0299$ & pcifs & 0.0622 & EPIA-01]B \\
\hline U] & $0.0017=0.008$ & $\mathrm{pCi}_{\mathrm{g}}$ & 0.0319 & EPLA-013 \\
\hline$x$ & $6.19+2.41$ & pcisg & 4,20 & E'L $1 \mathrm{~A}-00$ ? \\
\hline $\mathbf{x}$ & -0.6180 .097 & pCifs & $0,2\} 1$ & EPLA-003 \\
\hline $\mathrm{X}$ & $0.0019 \pm 0.005$ & pCirs & 0.0092 & EPLA- \\
\hline $\mathbf{x}$ & $0.05 * 6 \div 0.0128$ & $\mathrm{pCu}_{\mathrm{s}}$ & $0,0 \pm 23$ & EPLA-O13 \\
\hline$x$ & $0.00=0.00$ & pCig & 0.0441 & FP[A- -0$] 3$ \\
\hline$x$ & $0.0472 \div 0.049$ & pCrg & 0.102 & EPLA+006 \\
\hline$x$ & $139=0.165$ & pCirs & 0.0387 & EPLA-013 \\
\hline \multirow[t]{4}{*}{ تט } & $2.02 \div 0.973$ & pCifg & 1.53 & EPIA-0ZZ \\
\hline & $0.0937 \pm 0.0443$ & $\mathrm{pCi} / \mathrm{g}$ & 0.06 & EPLA-012 \\
\hline & 0.7840 .065 & $\mathrm{PCi} / \mathrm{g}$ & 0.0385 & EPA-013 \\
\hline & $525=0.469$ & PClits & 0.37 & EPLA-004 \\
\hline जा & $0.845 \pm 0.133$ & $\mathrm{pCi} / \mathrm{g}$ & 0.337 & EPIA-005 \\
\hline $\mathbf{x}$ & $0.654=0.107$ & $\mathrm{pev} / \mathrm{s}$ & 0.06 & $E[A A-0] 1$ \\
\hline
\end{tabular}

$\begin{array}{llllll}U & & 36.70 & \mu g / k g & 36.70 & \text { EPA8270 } \\ U & v & 14.30 & \mu / k g & 36.70 & \text { EPA8270 } \\ U & & 36.70 & \mu g / k g & 36.70 & \text { EPA8270 } \\ U & & 36.70 & \mu g / k g & 36.70 & \text { EPA8270 }\end{array}$

wykg 22.50

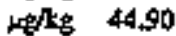

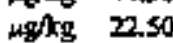

मfle 2.25
EPA8260

EPA 8260

EPA8260

EPA8260

F-Ares Retention Basin, Phase II 
SAMPLE NAME FRP+0803

Locemtion (SPS Coordinstes) $53670 \mathrm{E} 76 \mathrm{IS6} \mathrm{N}$

Smple Matrix: Sail
Sample 10: 10284

Interval Depthst 6,00 10 8.00

Percent Sobids: 82,00

Copstitucal

RQ AQ B Result

Unte D.Limit Method

Metas Paraneters

Assenic, lats] recovernofe

Barfum, sotat recoveroble

Beryllium, totsl recoverable

Chronium, tolal recoverible

Nickel, 1otal recoverable

Lead, toul socaverable

Thasifum, rotad recoverable

v

$\begin{array}{cc}\mathrm{v} & 6.42 \\ & \$ 1.40 \\ 0.467 \\ 10.60 \\ \\ \\ \\ \\ & 6.48 \\ & 6.42\end{array}$

Rodiological Paramelets

Gross alphs

Ameritiuso-241

Barium-133

Nomvolalile beta

Carbon-i4

Cotsit1-60

Cesium-137

Europinm-154

Iodine-129

Potssyiom-40

Nitkel-63

Plutonitrm-239/240

Raditim-226

Suontiars-90

Iochneilum 99

Uranium-23:

\begin{tabular}{|c|c|c|}
\hline & $x$ & $13.20 \pm 3.80$ \\
\hline & $x$ & $0.0384=0.0299$ \\
\hline & $x$ & $-0.04 \pm 0.0173$ \\
\hline & $\mathrm{x}$ & $58.80 \pm 5,31$ \\
\hline & $x$ & $-0.717=0.0968$ \\
\hline & $x$ & $-0.0031 \pm 0.0066$ \\
\hline & $x$ & $3.74 \leq 0,0556$ \\
\hline & $\mathbf{x}$ & $-0.0156+0.032$ \\
\hline & $x$ & $-0.0049 \div 0,072$ \\
\hline & $\mathbf{x}$ & $4,22 \pm 0,242$ \\
\hline & $v$ & $0.933 \div 0.372$ \\
\hline & $x$ & $0.0854 \pm 0.0346$ \\
\hline & $\mathbf{x}$ & $0.997 \pm 0.0769$ \\
\hline & $x$ & $23.29 \pm 1.79$ \\
\hline & $V X$ & 0.9240 .144 \\
\hline & $x$ & $1.05+0.195$ \\
\hline
\end{tabular}

\begin{tabular}{|c|c|c|}
\hline $\mathrm{i} / \mathrm{g}$ & $\begin{array}{l}.32 \\
.06\end{array}$ & EPI \\
\hline & 0.0186 & EPIA-OIJB \\
\hline & 4.02 & iB \\
\hline & 0332 & EP1A-003B \\
\hline & 0.01005 & EPLA-013B \\
\hline & 0.0155 & $E P 2 A-0+3 B$ \\
\hline Ci) & 0.0543 & 0138 \\
\hline 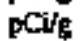 & 0.139 & EPLA-00\$B \\
\hline 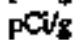 & 0.0503 & EPLA-0]3B \\
\hline$y$ & 0.721 & 02 \\
\hline $\mathrm{i}$ & 0.06 & Bלנים \\
\hline & 0.0494 & EPLA-013B \\
\hline & 134 & EPLA-00d \\
\hline & 0.395 & - EPLA-C0SE \\
\hline & 0.06 & EELA-OIIB \\
\hline
\end{tabular}

Seraivolatile Parameters

Dibenzofuran

Di-n-butyl phibalale

N-Nitrosadiphenylamine

2-Chlorophenol

$\begin{array}{llr} & & 9.49 \\ U) & \text { v } & 73.30 \\ U & & 39.50 \\ U & & 3950\end{array}$

Volatile Parmerers

Dichlorometiane (Melbyliane diloride)

Cribon disul|rde

Tolsenc

Trichlorothylene

$\begin{array}{lll}\text { J } & 0 & 0.951 \\ \text { UJ } & 0 & 1.22 \\ \text { uJ } & \text { vo } & 0.317 \\ \text { J } & 0 & 0.334\end{array}$

$\begin{array}{lll}\text { mp/kg } & 6.42 & \text { EPA6010 } \\ \text { mg/kg } & 3.21 & \text { EPA6010 } \\ \text { mg/kg } & 3.21 & \text { EPA6010 } \\ \text { mg/kg } & 3.11 & \text { EPA6010 } \\ \text { mg/kg } & 3.21 & \text { EPA6010 } \\ \text { mg/kg } & 6.42 & \text { EPA6010 } \\ \text { mg/kg } & 6.42 & \text { EPA6010 }\end{array}$

miks 39.50 EPA8270

Me/k. 3950 EPA8270

HEkg 3950 EPA8270

LAJkg 3950 EPAS270

$\begin{array}{lll}\mu q \mathrm{~kg} & 0.61 & \text { EPA8260 } \\ \mu / \mathrm{kg} & 1.22 & \text { EPA8260 } \\ \mu \mathrm{g} / \mathrm{kg} & 0.61 & \text { EPA8260 } \\ \mu / \mathrm{kg} & 0.061 & \text { EPA8260 }\end{array}$

F-Area Reiention Basin, Phase IJ 
SAMPLENAME: FRB-AS(19

LAetion (SRS Coordiartes): 53670 E $76156 \mathrm{~N}$

Sample Marix: Soil

Conștituent

Metrir Parareters

Asenic, total resorerrble

Batiom, toul recowerable

Beylliur, totril recomerabie

Chromian, 10tai recoverable

Nickel, wotal resoverobte

lesd, 1000l recoverble

Thatliutm, colal recowtrabite

Radialogidel Parandecters

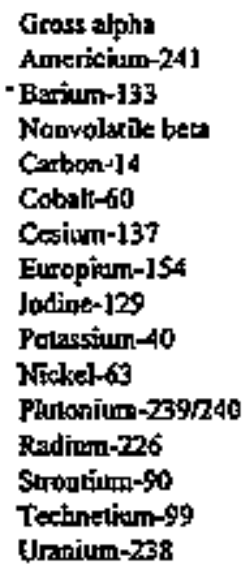

Semivolatile Paraserers

Dibenzofuren

Di-a-baryl phrbaleve

N-Nurosodiphenylumine

2-Chloraphenged

Votatik Parameders

Diahloromethane (Mfelbylene chiluride)

Carton disulfide

Tolusene

Trichlorocthylene
RO AQ $\mathrm{B}$

v

5.91
11.70
0.147
13.70
1.92
5.87
5.91

$\begin{array}{ll}\text { ⿰扌) } & x \\ \text { uj } & x \\ & x \\ \text { ul } & x \\ & x \\ \text { ul } & x \\ \text { ul } & x\end{array}$

unt

$X$
$X$
$X$

if $v x$

$x$

$x \quad 3630 \pm 6.25$

$0.0335 \pm 0.0506$

$0.0024=0.0184$

$274.00 \pm 11.50$

$-0.612 \div 0.0977$

$0.019 \div 0.0106$

J0230.0.098

$0.0104 \pm 0.0359$

$-0.0393 \pm 0.084$

$125=0.16$

L 2.78 \pm 0.97

$0.139 \pm 0.0457$

$0.506 \pm 0.071$

$102.00 \times 5.48$

$0.727 \pm 0.133$

$7.85 \pm 0.936$

4.09

$\begin{array}{lll}U & V & 18.20 \\ \mathrm{U} & & 3.20\end{array}$

U 3720

$\begin{array}{llll}\text { J } & 0 & \mathrm{H} & 0.416 \\ \mathrm{U} & 0 & \mathrm{H} & \$ .12 \\ \mathrm{U} & \text { VO } & \mathrm{H} & 0.18 \\ \mathrm{~J} & 0 & \mathrm{H} & 0.101\end{array}$

Sampte ID: 102285

Iuterval Depths: 8.00 to 10.00

Percent Solids: 89.00

\begin{tabular}{|c|c|c|}
\hline Unit & D.1+mic & Method \\
\hline malkg & 5.91 & EPAG \\
\hline mg/k & 2.96 & EPA60 \\
\hline$m g / k g$ & 2.96 & EPA6 \\
\hline migl & 296 & EPA60 \\
\hline m的 & 2.96 & EPAGO \\
\hline$m g h$ & 5.91 & EA60 \\
\hline 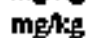 & $\$ \$ 1$ & EPA60 \\
\hline
\end{tabular}

\begin{tabular}{|c|c|c|}
\hline $\mathrm{Cit}$ & .74 & $\begin{array}{l}\text { EPLA-001B } \\
\text { EPIA-OL11B }\end{array}$ \\
\hline & 0.028 & EPLA+OLIB \\
\hline & 5.04 & EP \\
\hline & 0.212 & EPL \\
\hline & 0.0091 & EPIA-0I5E \\
\hline & 0.01 & EPI \\
\hline & 0.0544 & EPl \\
\hline & 0.146 & EPIA-OOSB \\
\hline & 0.0685 & EPLA+GTBE \\
\hline & 1.85 & EPIA-OZ2 \\
\hline & 0.06 & EPIA-0I2B \\
\hline & 0.0534 & EPLA+Ot3B \\
\hline & 0.367 & LA-004 \\
\hline & 0,338 & LA-005E \\
\hline & 0.061 & $P] A-0[1$ \\
\hline
\end{tabular}

$M \sigma / k E$ EPA3770

MEA:S 37.20 EPAS270

मEAKE 3720 EPA8270

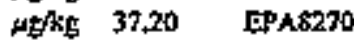

F-Atea Retemtion Batin, Phese it 
SAMPLENAME FRB-050S

Localion (SRS Coord'oles); 53670 E 76156 N

Sample Matrix: Soil

Constituent

Mebal Parameters

Arsenic, tolal recowerabie

Brilum, total recoverab]e

Beryllium, total recowatable

Chomitum, zomal recoverable

Nieket, 10001 resoverable

Lead, total recowable

Thallimm, 10tal recovesrable

Radbolagical Paramelers

Gross alphra

Antericium-241

Barium-133

Nonvolatile bets

Carbon-14

Cabali-60

Cosinam-137

Europium-1S4

lodine-129

Polassiura-40

Nickel-60

PJuroniun-229:240

Radium-226

Strontinm-90

Tochuetínmyg

Urenium-238

Seviwolitile Parametors

Dibenzofuran

Dintbutyil ptuhalste

N-Nitrosodighenylamine

2-Chiorophena!

Volexile Paramelers

Dichkromethane (Meihylene chlaride)

Cxabn disulfide

Toluese

Trichloroetbylene
$\mathrm{RQ} \mathrm{AQ} \mathrm{B}$ inerult

Sampic iD: 1022\$6

Interval Depcis: $10.00 \mathrm{kp} 12.00$

Percent Sobids: 87.00

Unth D.Linit Method

$\mathbf{u}$

2.14
5.15
0.119
1750
1.44
6.69
6.05

\begin{tabular}{|c|c|c|}
\hline mglt & 6,05 & EPA 6010 \\
\hline gi & 3.02 & EPA6P]0 \\
\hline & 3.02 & EPA6010 \\
\hline & 3.02 & EPAGOJO \\
\hline & 3.62 & EPA60to \\
\hline & 6.05 & EPA6010 \\
\hline & 6. & EPASOto \\
\hline
\end{tabular}

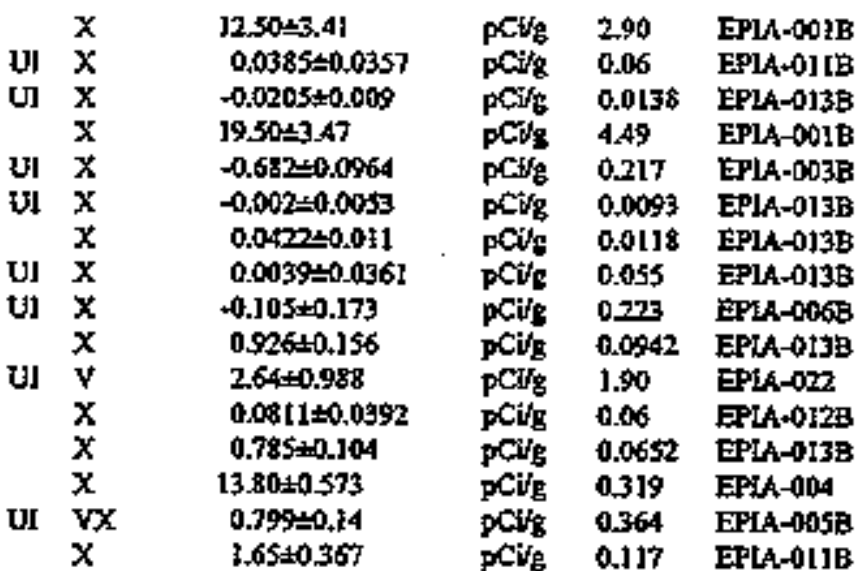

$\begin{array}{lll}u & & 37,70 \\ u & v & 13.60 \\ u & & 37.70 \\ u & & 37.70\end{array}$

$20 / \mathrm{kg} \quad 37.70$

mefk 37.70

$\mu g / \mathrm{kg} \quad 37.70$

$\mu \mathrm{g} / \mathrm{kg} \quad \mathbf{3 7 . 7 0}$

EPA8270

EPAB270

EPA8270

도A8270

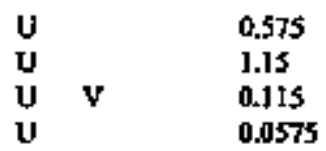

$\begin{array}{lll}\mu \mathrm{gkg} & 0.575 & \text { EPAS660 } \\ \mu \mathrm{kg} & 1.15 & \text { EPA8260 } \\ \mu \mathrm{g} / \mathrm{kg} & 0.575 & \text { EPA8260 } \\ \mu / \mathrm{kg} & \mathbf{0 . 0 5 7 5} & \text { EPA8260 }\end{array}$

F-Area Retention Basin, Ftose I] 
SAMPLENAME: FRB-0806

locastont (SRS Courdinalt5): 53670 E $76156 \mathrm{~N}$

\$ample Marix: Soil

Constituent

Measl Paramelers

Assenic, 1otal recoreratis

Esrinim, zocal rexpyefatile

Beryilinum, totes recowerible

Cheropainter, zosal roxowertable

Nickel, total resoverpitte

Lead, rotal recovtrabls

Thallium, intal recoverable

Radiologikal Parameters

Gross alphe

Amerixium-24!

Barion-133

Noswatotile bees

Carbon-14

Cobali-60

Cesfan-137

Europium-154

ladine-129

Potassium-40

Nidel- 63

Piuconimm-229/240

Radium-286

Stromium-90

Techueriura 49

Urnium-238

Semivalatile Parameters

Dibenzofuran

Di+z-butyi phihalace

13-Nitrosodiphenytamine

2-Chloroment!

Vokulle Paramelets

Dicblorofatubine (Mehlylente chlorids)

Cribor disulfitse

Toluente

Trieblorotbylene
RQ AQ B

Interval Depths. 12,00 to 14.00

Percent Solits: 86.00

Uni

D.Limit Method

U

$\begin{array}{cc} & 7.13 \\ & 4.44 \\ 0.094 \\ 23.34 \\ 1.12 \\ 7.56 \\ 6.12\end{array}$

$\begin{array}{ll}\text { mg/kg } & 6.12 \\ \text { megk } & 3.06 \\ \text { mg/kg } & 3.06 \\ \text { me/kg } & 3.06 \\ \text { mg/kg } & 3.06 \\ \text { me/kg } & 6.12 \\ \text { mg/kg } & 6.12\end{array}$

EPA 6010

EPAO010

EPA6010

EPADOTO

EPAGT10

EPA6010

EPASOIO

\begin{tabular}{|c|c|c|c|c|c|}
\hline & $\begin{array}{l}x \\
y\end{array}$ & $1520=3.80$ & $\mathrm{pCi} / \mathrm{s}$ & 2.92 & EPLA-ADIB \\
\hline Uj & $\mathrm{x}$ & $0.0318+0.0358$ & poivs & 0.06 & EPLA-0IJE \\
\hline \multirow{2}{*}{$\mathbf{U :}$} & $x$ & $0.0056=0.0089$ & poifs & 0.0139 & EPIA-013B \\
\hline & $x$ & $1420=3.14$ & pCils & 4.50 & EPJA-001E \\
\hline Ui & $x$ & $-0.53 \pm 0.10$ & $p C t_{g}$ & 0231 & EPLA $005 B$ \\
\hline \multirow[t]{2}{*}{ Ut } & $x$ & $-0.0016=0.0053$ & pCifs & 0.0091 & EPLA-0I3E \\
\hline & $x$ & $0.115 \pm 0.0182$ & pCifg & 0.0126 & EPIA-0I3E \\
\hline ul & $x$ & $0.0072 \leq 0.0346$ & pCirs & 0.0517 & EPLA-013B \\
\hline \multirow[t]{2}{*}{$\mathbf{U F}$} & $\mathrm{x}$ & $0.0535=0.07$ & $\mathrm{pCV} / \mathrm{g}$ & 0.116 & EPLA-AOGB \\
\hline & $x$ & $1.01 \pm 0.14$ & peifs & 0.0025 & EPLA-0I3B \\
\hline \multirow[t]{4}{*}{ UI } & $v$ & $1.74 \pm 0.559$ & $\mathrm{PCi}$ & 1.05 & EPLA-027 \\
\hline & $\mathbf{x}$ & 0.062240 .0371 & $\mathrm{SO}^{\prime}$ & 0.06 & EPLA $012 \mathrm{E}$ \\
\hline & $x$ & $0.73150 .1 \pm t$ & p & 0.0673 & EPLA-0]3B \\
\hline & $x$ & $8.35 \pm 0.569$ & $p w_{\text {官 }}$ & $0 A 06$ & EPIA-004 \\
\hline \multirow[t]{2}{*}{ บ } & $\sqrt{x}$ & $0.957 \pm 0.147$ & pers & 0.374 & EPLA-0OSE \\
\hline & $\mathrm{x}$ & $1.20 \pm 0.229$ & pcirg & 0.0713 & IFIA-0] IB \\
\hline
\end{tabular}

$\begin{array}{lll}u & & 38.20 \\ u & V & 1490 \\ y & & 38.20 \\ U & & 38.20\end{array}$

$\mu 8 / \mathrm{kg} \quad 35.20$

$18 / k \mathrm{~kg} 3820$

मgke 3820

$\mu \mathrm{fkg} \quad 38.20$

EPAS270

EPA8270

EPABZ70

EPA8270

$\begin{array}{lll}\mu E / k g & 0.5 \$ 1 & \text { EPA8260 } \\ \mu g / k g & 1,16 & \text { EPA8260 } \\ \mu \mathrm{g} / \mathrm{kg} & 0.581 & \text { EPA8260 } \\ \mu \mathrm{g} / \mathrm{kg} & 0.0581 & \text { EPA8260 }\end{array}$

F+Area Relemion Basin, Phast II 


\begin{tabular}{|c|c|c|c|c|c|c|c|}
\hline \multicolumn{4}{|c|}{$\begin{array}{l}\text { SAMPLE NAME: FRE-1901 } \\
\text { Location (SRS Coordinates). } 53742 \text { E } 76253 \mathrm{~N} \\
\text { Sample Marix Soil }\end{array}$} & \multicolumn{4}{|c|}{$\begin{array}{l}\text { Sample ID: } 102257 \\
\text { ImtervalDeputs: } 0.00 \text { wo } 1.00 \\
\text { Percent Solids. } 86.00\end{array}$} \\
\hline Canstituent & $\mathbf{R Q}$ & AQ & B & Resalt & Unit & D.Limit & Method \\
\hline \multicolumn{8}{|l|}{ Melal Paramerers } \\
\hline 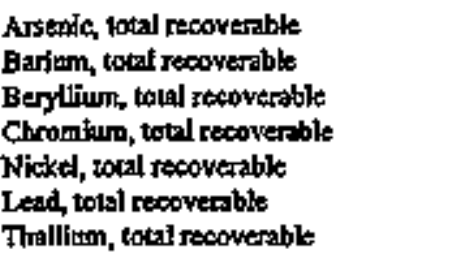 & $\mathbf{U}$ & & & $\begin{array}{c}6.12 \\
29.40 \\
0.315 \\
13.60 \\
3.93 \\
6.89 \\
6.12\end{array}$ & $\begin{array}{l}m g / k g \\
m g / k g \\
m g k k g \\
m g i k g \\
m g k k g \\
m g / k g \\
m g k k g\end{array}$ & $\begin{array}{l}6.12 \\
3.06 \\
3.06 \\
3.06 \\
3.06 \\
6.12 \\
6.12\end{array}$ & $\begin{array}{l}\text { EPA6010 } \\
\text { EPA6010 } \\
\text { EPA6010 } \\
\text { EPA6010 } \\
\text { EPA6010 } \\
\text { EPA6010 } \\
\text { EPA6010 }\end{array}$ \\
\hline \multicolumn{8}{|l|}{ Radiolggifcal Parameleters } \\
\hline 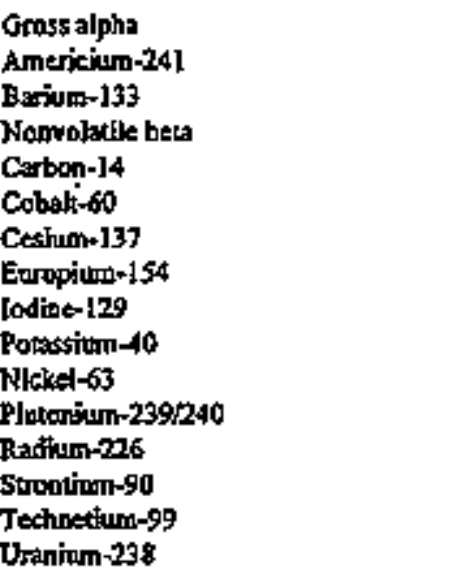 & $\begin{array}{l}\text { U] } \\
\text { U] } \\
\text { U] } \\
\text { U! } \\
\text { UI } \\
\text { Ut } \\
\text { uI } \\
\mathbf{J} \\
\text { UI } \\
\text { UI } \\
\text { uI } \\
\mathbf{J}\end{array}$ & $\begin{array}{l}\mathrm{v} \\
\mathrm{c} \\
\mathrm{v} \\
\mathrm{c} \\
\mathrm{c}\end{array}$ & & $\begin{array}{c}27.60 \pm 5.02 \\
0.0300=0.0277 \\
-0.0514 \pm 0.0048 \\
8375 \pm 2.72 \\
-0.002 \pm 0.0871 \\
0.0916 \pm 0.0031 \\
0.118 \pm 0.0073 \\
-0.003 \pm 0.0192 \\
0.057 \pm 0.075 \\
1.24 \pm 0.0892 \\
1.69=0.529 \\
0.0044 \pm 0.0205 \\
0.791 \pm 0.0632 \\
-0.27 \pm 0.247 \\
0.215 \pm 0.107 \\
0.936 \pm 0.146\end{array}$ & 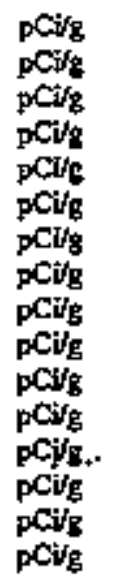 & $\begin{array}{l}2.77 \\
0.06 \\
0.057 \\
4.50 \\
0.174 \\
0.0054 \\
0.0064 \\
0.028 \\
0.118 \\
0.0519 \\
0.99 \\
0.06 \\
0.0405 \\
0.283 \\
0.294 \\
0.06\end{array}$ & $\begin{array}{l}\text { EPLA-001B } \\
\text { EPLA-01IB } \\
\text { EPIA-013B } \\
\text { EPLA-001B } \\
\text { EPLA-003B } \\
\text { EPLA-013B } \\
\text { EPLA-013B } \\
\text { EPLA-13]3B } \\
\text { EPLA-006B } \\
\text { EPIA-013B } \\
\text { EPIA-022 } \\
\text { EPLA-012B } \\
\text { EPIA-013B } \\
\text { EPIA-004 } \\
\text { EPLA-005B } \\
\text { EPIA-011B }\end{array}$ \\
\hline \multicolumn{8}{|l|}{ Seminolatila Parameters } \\
\hline 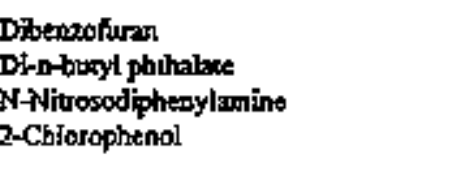 & $\begin{array}{l}\mathbf{U} \\
\mathbf{U} \\
\mathbf{U} \\
\mathbf{U}\end{array}$ & $v$ & & $\begin{array}{l}37.90 \\
28.10 \\
37.90 \\
37.90\end{array}$ & $\begin{array}{l}\mu g / \mathrm{kg} \\
\mu \mathrm{kg} \\
\mu / \mathrm{kg} \\
\mu / \mathrm{kg}\end{array}$ & $\begin{array}{l}37.90 \\
37.90 \\
37,90 \\
37.90\end{array}$ & $\begin{array}{l}\text { EPA8270 } \\
\text { EPA8270 } \\
\text { EPA8270 } \\
\text { EPA8270 }\end{array}$ \\
\hline \multicolumn{8}{|l|}{ Volatile Paramezers } \\
\hline 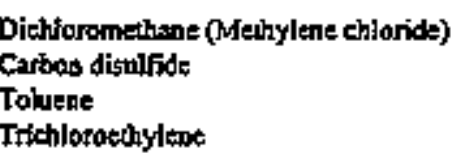 & $\begin{array}{l}u \\
y \\
w\end{array}$ & $\begin{array}{l}\text { vo } \\
0 \\
\text { veot } \\
0 \quad\end{array}$ & $\begin{array}{l}\mathbf{H} \\
\mathrm{H} \\
\mathrm{H} \\
\mathrm{H}\end{array}$ & $\begin{array}{l}0.837 \\
1.16 \\
0.314 \\
0.453\end{array}$ & 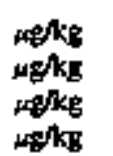 & $\begin{array}{l}0.581 \\
1.16 \\
0.581 \\
0.0581\end{array}$ & $\begin{array}{l}\text { EPAB260 } \\
\text { EPA8260 } \\
\text { EPA\$260 } \\
\text { EPA } \$ 260\end{array}$ \\
\hline
\end{tabular}

FrArea Rexention Basin, Phase I] 
SAMPLE NAME: FPB-i912

Locstion (SRS Coordinates): 59742 E $76253 \mathrm{~N}$

Sampite Matrix: Sofl

Constituent

Metal Parameters

Afsedic, rotal recowerabls

Barium, total rocoverablo

Berylfinth, 1otal recoverable

Chromium 10tal recovertole

Nitickel, rolal recoverabite

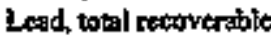

Thallium, totol recoverabte

Radiologital Paranteters

Gross alpha

Ameticium-241

Bariurn-\{ 93

Norwolutile beta

Carbon-14

Cobati-60

Cesinm-137

Europium-154

Codint-229

Potassom-40

Nicket-63

Fiutonitm-239/240

Radian-226

Svontium-90

Techeltium-99

Uraniutor-23:

Semrvolatite Paramerers

Dibenzofistan

Di-n totyl phuhdale

N-Nitrosodiphenylamine

2-Chloropherol

Volatile Parameters

Dichlaromethane (Methylene chloride)

Carbon disulkide

Toluene

Trichloroelloylene
RQ AO B Besult

Uait

D.Limit Method

ㄴ.

6.05

15.80

0.16

13.10

257

4,70

U

6.05

Sarpile ID: 102758

Interval Depthst 10.001012 .00

Percent Solids 87.00

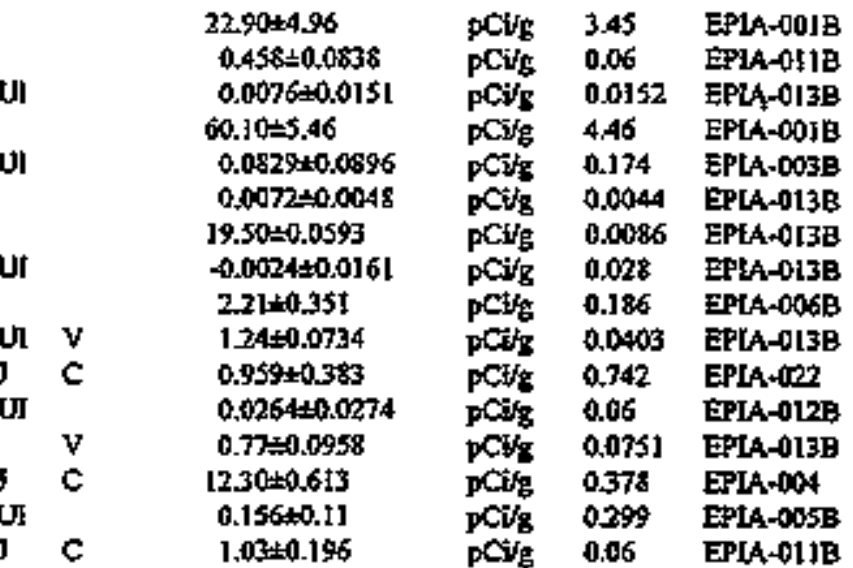

$\begin{array}{lll}u & & 37.40 \\ U & V & 34.40 \\ U & & 37.40 \\ U & & 37.40\end{array}$

$\begin{array}{lll}\mu \mathrm{g} / \mathrm{kg} & 37.40 & \text { EPA8270 } \\ \mu \mathrm{B} / \mathrm{kg} & 37.40 & \text { EPA8270 } \\ \mu D / \mathrm{kg} & 37.40 & \text { EPA8270 } \\ \mu \mathrm{k} / \mathrm{kg} & 37.40 & \text { EPAS770 }\end{array}$

$\begin{array}{llll}\text { U } & \text { VO } & H & 0.62 I \\ \text { U } & 0 & H & 1.15 \\ \text { UJ } & \text { V8. } & H & 0.172 \\ \text { U } & 0 & H & 0.057\end{array}$

4 C $\mathrm{kg} \quad 0575$ EPA8260

$\mu / \mathrm{kg} \quad 1.15$ EPA8260

$\mu$ glk 0.575 EPA8260

$\mu \mathrm{g} / \mathrm{k}$ 0.0575 EPA8260

F-Area Relention Basth, Phase II 
SAMPLE NAME FRB-1909

Loction (SRS Coordirates): 53742 E 76253 N

Simple Marix: Soil

Constitnent

Metal Pramciers

Arsenic, 10tal tecoverable

Beritran, rocal rewowable

Beyllian, total necoverabic

Chominu, toos recovirable

Nickel, lotal recoverable

led, rotal tecoverablo

Thatliurn, totil reorverable

Radiologinga\} Paramerers

Gros alphz

Americitsm-24I

Barium-133

Nonvalatile bets

Cubon-14

Cobplt-60

Ce-ium-137

Europitum-154

Iodin-123

Potrissium 40

Nickel-63.

Protonium-239/240

Radium-26

Smontium-90

Technetion 49

Uranium-235

Sentivolatile Parameters

Dthenzenfura

Dis-batyl phuhalate

N-Niacosodiphenylamine

2-Chiorophenol

Voletile Parameters

Dichlorometharte (Adetbyitene chloride)

Carbent disulfide

Tolvene

Trichlocoteryitene
RQ AQ B Result

Simple to: 102259

Inierval Depths: 12.00 to 14.00

Percem Soliós. 88.00

D.Limit Method

t

$$
\begin{gathered}
5.98 \\
t 130 \\
0.164 \\
12.40 \\
2.17 \\
6.86 \\
5.98
\end{gathered}
$$

U

$\begin{array}{lll}m g / k g & 5.98 & \text { EPA6010 } \\ m / k g & 2.99 & \text { EPA6010 } \\ m g / k g & 2.99 & \text { EPA6010 } \\ m g / k g & 2.99 & \text { EPA6010 } \\ m g / k g & 2.99 & \text { EPA6010 } \\ m / k g & 598 & \text { EPA6010 } \\ m g / k g & 5.98 & \text { EPA6010 }\end{array}$

\section{U1}

U1

U]

U3

Ut

$\begin{array}{ll}\text { UI } & \\ \text { UI } & \\ \text { UI } & v \\ \text { J } & C \\ \text { UI } & \\ \text { UI } & v \\ \text { J } & c \\ \text { ur } & \\ \text { J } & c\end{array}$

$-0.574 \leq 1.43$
$0.007 \pm 0.0261$
$-0.009=0.0136$
$0.682 \pm 1.73$
$0.061=0.0867$
$0.0257=0.0054$
$35.50 \pm 0.088$
$-0.144=0.0189$
$0.00 \pm 0.06$
$0.89 \pm 0.0759$
$1.11 \pm 0.341$
$0.0319 \pm 0.0321$
$0.582 \pm 0.194$
$99.10 \pm 1.32$
$0.108 \pm 0.0999$
$0.555 \pm 0.143$

pcits

4.36

$\mathrm{PCi} / \mathrm{g} \quad 0.06$

ocis $\quad 0.022$

pCits 3.99

pCigg 0.167

pCig 0.0048

pCis 0.0121

pCis 0.0255

PCig 0.234

pCis 0.0439

pCig 0.637

PCy $\quad 0,06$

PCE 40.143

$\mathrm{pCl} / \mathrm{g} \quad 0319$

PCF/s 027

$\mathrm{PC} / \mathrm{s}$

0.0721

EPLA-00IB

EP $A-A) 1 B$

EPUA-013B

EPLA-001B

EPIA-0D3B

EPLA-013B

EPIA-0I3B

EPLA-013B

EPLA-006B

EPLA-013B

\begin{tabular}{|c|c|c|c|c|}
\hline $\begin{array}{ll}u & \\
u & v \\
u & \\
u & \end{array}$ & $\begin{array}{l}37.00 \\
28.10 \\
37.00 \\
37.00\end{array}$ & $\begin{array}{l}\mu / \mathrm{kg} \\
\mu / \mathrm{kg} \\
\mu g / \mathrm{kg} \\
\mu / \mathrm{kg}\end{array}$ & $\begin{array}{l}37.00 \\
37.00 \\
37.00 \\
37.00\end{array}$ & $\begin{array}{l}\text { EPAB270 } \\
\text { EPAB270 } \\
\text { FPAB270 } \\
\text { EPAS770 }\end{array}$ \\
\hline
\end{tabular}

EPIA-022

EPLA D12B

EPLA-013B

EPIA -004

EPLA-OOSB

EPIA-OIIB

F-Arta Retention Easto, Phese II

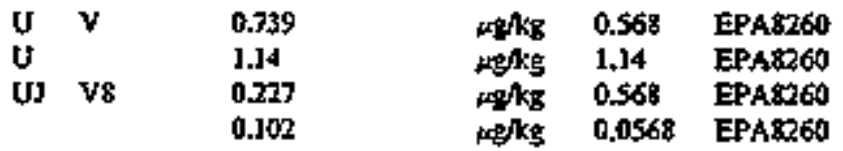


SAMPLENAME: FRR-1904

Locrtion (SRS Condimates); 53742 E $76253 \mathrm{~N}$

Sample Matrix: Solt

Coossitoent

RQ AQ B Rerult

Sample ID: 102060

Interval Depths [4.00 10 16.90

Percent Solids: 85.09

Metal Pagrelers

Arsenic, total scoowerable

Batoum, 10tal recoverable

Beyllitsm, watal recoredabte

Chrominm, sotal recorverible

Nícket, 10wl tecoversble

Lead, total resoverable

Thallum, tolal frocowerable

Radiologieal Parameters

Gross alpha

Americium-24]

Barinon-133

Norvolatie beta

Carbon-tid

Cob:aj -60

Cofíma-13?

Europinum-154

Jodiue-125

Potsesium-10

Nickete63

Ptutomitum-239/240

Fadhm-2026

Strontium-90

Technetiun -99

Uraniam-238

Senivolatite Parameters

Dibenzotaran

Di-m-tutyl phuhaigte

N-Niurcsodiphenylaprine

2-Chlorophenel

Volatile Parametcrs

Dichlorowe thane (Methylent chiloride)

Carbon distrifide

Tolvenes

Trichloroeshylene

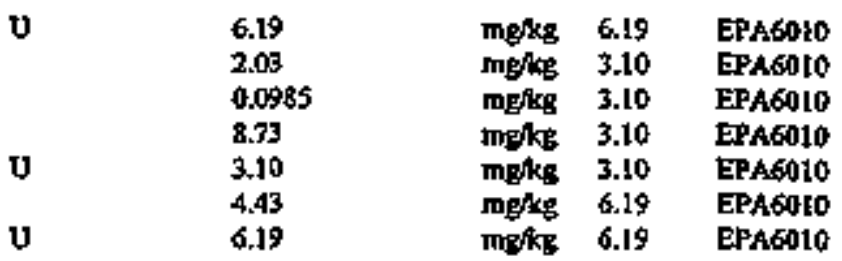

$16.20 \pm 5.32$

$\mathrm{pC} / \mathrm{s} \quad 4.36 \quad$ EPIA-0NJB

PCits 0.06 EPLA-01]B

pCirs 0.0182 EPLA-013B

${ }_{\mathrm{pCi}} \mathrm{C} \quad 4,19$ EPLA-00]E

pCitg 0.171 EPLA-005B

U]

$0.0061 \neq 0.0128$

$464.00 \pm 14,30$

$0.109 \pm 0.0868$

$0,0132=0.0067$

11. $.50 \pm 0.0519$

$-0.142-0.0313$

$0.0118 \pm 0.163$

$3.04 \pm 0.12]$

$1,20 \pm 0.46$

$0.4244 \pm 0.04 \$ 6$

$0.934 \pm 0.18$

$275.00 \pm 2.12$

$0.0775 \pm 0.103$

$0.608 \pm 0.573$

$\mathrm{pCi}$ is $_{\mathrm{s}}$

0.0067

EPLA-013B

pCist 0.0116 EPLA-013B

pCits 0.0398 EPLA-0I3B

pCi's 0.301 EPLA+006B

pCi's 0.0673 EHA-013B

pCis 0.772 EPLA-1022

DCis 0.07s EPLA+0]2B

PCifs 0.117 EPLA-013B

PCi's $0.289 \quad$ EPLA-004

CWig 0285 EPLA-005B

j $c$

$\mathrm{pCi} / \mathrm{s}$

0.0644

EPLA+0) IB

$\begin{array}{lll}U & & \\ \text { U } & V & 38.60 \\ \text { U } & & 25.10 \\ \text { J } & & 38.60 \\ & & 38.60\end{array}$

$6 / \mathrm{kg} \quad 3860$

tof 38.60

$\mu g / \mathrm{kg} \quad 38.60$

लAks: 38.60

EPA8270

포A 8270

EPA8270

EPA3270

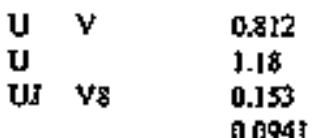

and

0.588

EPA8260

$\omega \mathrm{g} / \mathrm{kg}$ I.18 EPA8260

$\mu t / \mathrm{kg}$ 0.588 EPA8260

$\mu g / \mathrm{kg} 0.0583$ EPA8260

F-Area Recenuion Basin, Phast I] 
SAMPLE NAME: FRB-190S

Localion (SRS Coondinates)t 53742 E76253 N

Sample Matrix: Soit

Consticuent

Metal Paraneters

Arsenic, rotal recoverable

Barium, solal recoverable

Beryllisu, 100al recoverrable

Clonomium, lots recovernble

Nickel, 10al tecorvatable

Lead tot recoverable

Thalifirm, zolal recorqunible

Radiolegical Parameters

Grass alphta

Amerieiurm-24]

Batium- 133

Nonvolatile bets

Cribon-14

Cobalr-60

Cesiun-137

Europiun-154

lodisue 129

Potarsinim-40

Niditer 63

Plutonium-239/240

Rafium-226

Surontiom-90

Technetiun-99

Utranjum-238

Sennivolatile Paramoters

Dibenzofiuran

Di-s-butyl phothalate

N-Nitrosodiphenylanine

2-Chiorophenal

Volatile Parameters

Dichloromethane (Melhylene chloride)

Carton disultidis

Toluene

Trikblanotibyitenc
Sarpile 15: $10726 \mathrm{~L}$

interval Depths: 16.00 to 18.00

Percent Solids 84.00

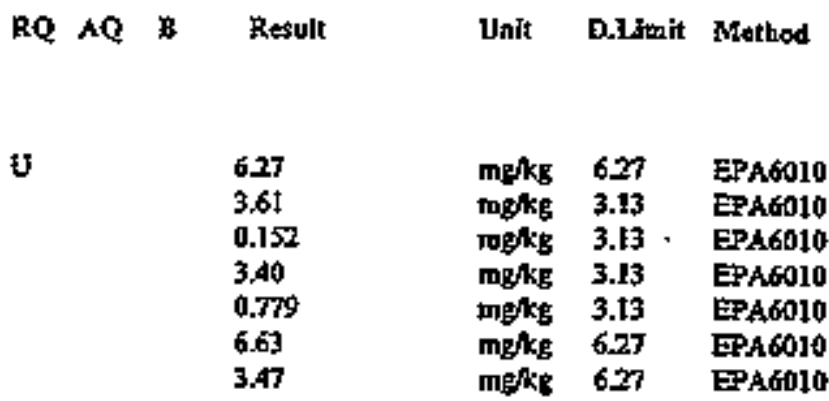

\begin{tabular}{|c|c|c|c|c|c|}
\hline \multirow[b]{2}{*}{ U] } & & $17.60-4.87$ & $\mathrm{pCit}$ s & 3.17 & EPIA-ONIB \\
\hline & & $0.0057 \pm 0.0394$ & pCis & 0.06 & EPIA-Q[1B \\
\hline \multirow[t]{2}{*}{ U] } & & $-0.0308 \pm 0,0997$ & $\mathrm{pCing}$ & 0.0144 & EPLA-0|3B \\
\hline & & $571.00 \pm 16.20$ & ocos & 4,06 & EPAA-OOIB \\
\hline \multirow[t]{3}{*}{ Ut } & & $0.0875 \pm 0.0864$ & $\mathrm{pcis}$ & 0.173 & EPLA-013B \\
\hline & & $0.0112=0.0057$ & pestg & 0.0066 & EPLA-OI3B \\
\hline & & $193=0,0233$ & pCitg & 0.0108 & EPA-013B \\
\hline & & $-0.0250=0.0285$ & $\mathrm{pCt} / \mathrm{s}$ & 0.0412 & EPLA-013B \\
\hline U & & $-0.0857=0.137$ & $\mathrm{pCi} / \mathrm{s}$ & 0.28 & EPLA-006B \\
\hline $\mathbf{U}$ & $v$ & $2.55 \pm 0.13$ & pCig & 0.0512 & EPLA $013 B$ \\
\hline$I$ & $c$ & $1.92 \div 0.407$ & $\mathrm{pCi} / \mathrm{g}$ & 0.706 & EPIA-AZ \\
\hline & & $0.003 \pm 0,0059$ & $\mathrm{pCi}$ & 0.06 & EPI $4012 \mathrm{~B}$ \\
\hline & $v$ & $1.05 \pm 0.10$ & $\mathbf{p} C t_{4}$ & 0.0664 & EPLA-013B \\
\hline 1 & $c$ & $305.00=227$ & $\mathrm{pCit}$ & 0299 & EPHA-004 \\
\hline & & (0. $182 \pm 0.114$ & pCt & 0.321 & EPLA-0OSB \\
\hline & c & $0.427=0.12$ & $\mathrm{pCi}$ & 0.06 & EPAA-01:B \\
\hline
\end{tabular}

$\begin{array}{llll}\text { Ut } & Q & & 39.20 \\ \text { W } & Q & & 39.20 \\ \text { UJ } & Q & & 39.20 \\ \text { J } & Q & & 13.30\end{array}$

H영 39 ב20

wolk 39.20

$\mathrm{HF} / \mathrm{kg} \quad 3920$

$\mathrm{cos} \quad 3920$

EPAEZ70

EPABZ70

EPA8230

EPA8770

F-Area Retetrion Basin, Phase II

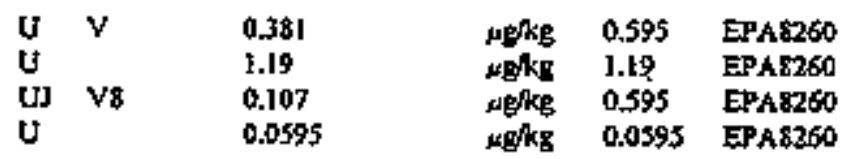


SAMPLENAMIE: FRB-1906

Location (SRS Coordinales); 53742 E 76353 M

Sernple Matrix: Soji

Constitutnt

Sample 1D: 10266

lenterval Depuls: 18.00 to 20.00

Pereent Soldss 83.00

\section{Metal Parineters}

Arsenic, total recoverale

Barium, 10:9l recoverible

Berylliop, tocal recowterte

Chromium, tont peoverible

Njickel, minl recoverable

Lood, rotal reeoperable

Thsllium, total necoverable

Radiological Parameress

\section{Gross alpha}

Americiurn-24]

Barrium-133

Nasyolatile beta

Cintop. 14

Cobsit-60

Cositurt-197

Etropium-2:54

joditis-129

Patassium-40

Nickpl-63

Putonim-239240

Radiun. 226

Stroutium-90

Technetum-99

Utanitum-238

Semivolatile Paramelers

Dibenzoptirn

Dj-aboxyl phthalats

N-Nitrosodiptenyiamine

2-Chlorephessal

Volalile Paraneres

Dichloromethane (Methylene chloride)

Caftoon disollide

Toluene

Trishlowedrylare
RQ AQ B Restlt

Uaft

D.Limit Method

U

6.34

1.68

0.137

4.08

0.912

5.46

U

634

UI

UI

$10.50 \pm 3.90$

$0.0099=0.0256$

$-0.0013 \pm 0,0078$

$712.00 \pm 1$ * 20

$0.102=0.0895$

$0.003]=0.0036$

$0.202 \pm 0.01$ is

$0.0245 \pm 0.027$

$-0.0867 \pm 0.131$

$1.39 \pm 0.104$

I.4540.453

$0.0265 \pm 0.0195$

$0.952 \pm 0.1[2$

$327.00 \pm 2.43$

$0.0429 \times 0.0987$

$0.387+0.115$

$\begin{array}{ll}\text { mg/kg } & 6.34 \\ \text { mgfkg } & 3.17 \\ \text { me/kg } & 3.17 \\ \text { mefks } & 3.17 \\ \text { mekg } & 3.17 \\ \text { miks } & 6.34 \\ \text { mgAkg } & 6.34\end{array}$

EPAEOJO

EPAGOIO

EPA6010

EPA6010

EPAG010

EPA6010

EPA6010

UI

J C

v1

J C

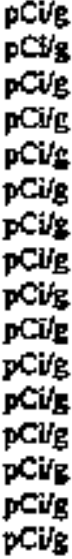

0.623

EPIA-001B

0.06

0.0314

GPAADIIE

EPLA-013B

EPLA-001B

0.181 EPIA-003B

0.0063 EPLA-0E3B

0.0087 EPLA-013B

0.0353 EPLA-033B

0.271 EPLA-006E

0.0552 EFLA-013B

0.848 EPLA-022

O.06 EFIA-012B

0.0793 EPLA-0138

0.319 EPIA-004

0.257 EPLA-005B

0.0631 EPIA-011E
Mefks 39.70

HJk 39.70

MERE 39.70

$\mu / \mathrm{kg} \quad 39.70$

EPAS270

EPA8270

EPA8230

EPAS270

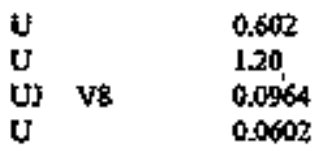

EPA8260

EPA 260

EPA2260

0.0602 EPA\$260

\section{HEN}

$\mathrm{arg} / \mathrm{ks}$

ugers

$\omega \mathrm{g} / \mathrm{kg}$

F-Ares Redention Basin, Phzse II 
SAMPLE NAME: FRZ-1907

Locution (SRS Coordinates): 53742 E $76253 \mathrm{~N}$

Sample Matrix: Sotl

Corstifuent

Metal Paramclers

Arsenic, Lotal recoverable

Barium, tolal recoverrable

Beryllium, cotal secoverable

Chrouidu, toks Jetoreabli

Nfckel, total recovecable

Lead total recover able

Thalleum, total recoverable

Radiological Parameters

Gross alpha

Aracricinam-243

Bariam-133

Nonvobtile beta

Carbon-14

Cobalt-60

Cesium-137

Europium-154

lodint-129

Polassium 40

Niekel-63

Fluronílum-239/240

Rodinam-226

Suromtitumego

Techoelitun-99

Utomium-238

Somivoletile Parameters

Diberzofuran

Di-n-butlyl phlhalare

N-Witrosodiphenylagine

2-Chlorophengl

Volatile Parametirs

Dichlofonedhane (Mdethylebe chloride)

Carbon disulide

Tolvene

Trichlorosthylepe u

5.\$1

256

0.157

4.52

0.626

6.30

y

5.93

$12.00 \pm 3.63$ $0.0196 \pm 0.0336$ $0.0011 \pm 0.005$

$394.00 \pm 13,80$

$0.0274 \pm 0.0842$

$0.0026 \pm 0.0025$

$0.249=0.0083$

$-0.0845+0.0164$

$-0.0191 \pm 0.09$

$0.900 \div 0.0747$

$0.925+0.361$

$0.0002 \div 0.0101$

$0.369 \div 0.10$ !

$202.00 \pm 2.19$

$0.0957 \pm 0.111$

$0.394 \pm 0.112$
Sample 2D: 102263

inierval Depths: 20.00 lo 22.00

Percent Solids: 89.00

Unic D.Limit Method

$\begin{array}{lll}\mathrm{mg} / \mathrm{kg} & 5.91 & \text { EPA6010 } \\ \text { mo/kg } & 2.96 & \text { EPA6010 } \\ \mathrm{mg} / \mathrm{kg} & 2.96 & \text { EPA6610 } \\ \mathrm{mg} / \mathrm{kg} & 2.96 & \text { EPA6010 } \\ \mathrm{mg} / \mathrm{kg} & 2.96 & \text { EPA6010 } \\ \mathrm{mg} / \mathrm{kg} & 5.91 & \text { EPA6010 } \\ \mathrm{mg} / \mathrm{kg} & 591 & \text { EPA6010 }\end{array}$

pCi's 1.71 EPIA-00IB

pCi/g 0.06 EPLA-01 IB

pCits 0.0076 EPLA-013B

pCi/g 3.98 EFLA+0OIB

PCis 0.162 EPLA-003B

$\mathrm{PCi} / \mathrm{g} \quad 0.0045$ EPLA-613E

PCi/E 0.0055 EPIA-013B

PCig 0.024] EP[A-013B

PCIf 0.176 EFLA-0DSB

pCiE 0.035 EPLA-013B

$\mathrm{PCH} / \mathrm{g} \quad 0,698$ EPLA-022

pCis 0.06 EPLA-0t2B

pCis 0.0749 EPLA-0:3B

$\mathrm{pCD} / \mathrm{g} \quad 0.416 \quad$ EPLA+O04

pCis 0.298 . EPLA-005B

PCilg 0.06 EPIA-011B

HAkT 37.30 EPA5270

Helk: 3730 EPAB270

$\mu g / \mathrm{kg} \quad 3730$ EPA8270

pokg 3730 EPA8270

37.30
2720
3730
3730

0.562

1.12

0.0787

0.0562

F-Area Retention Basin, Phase 1] 


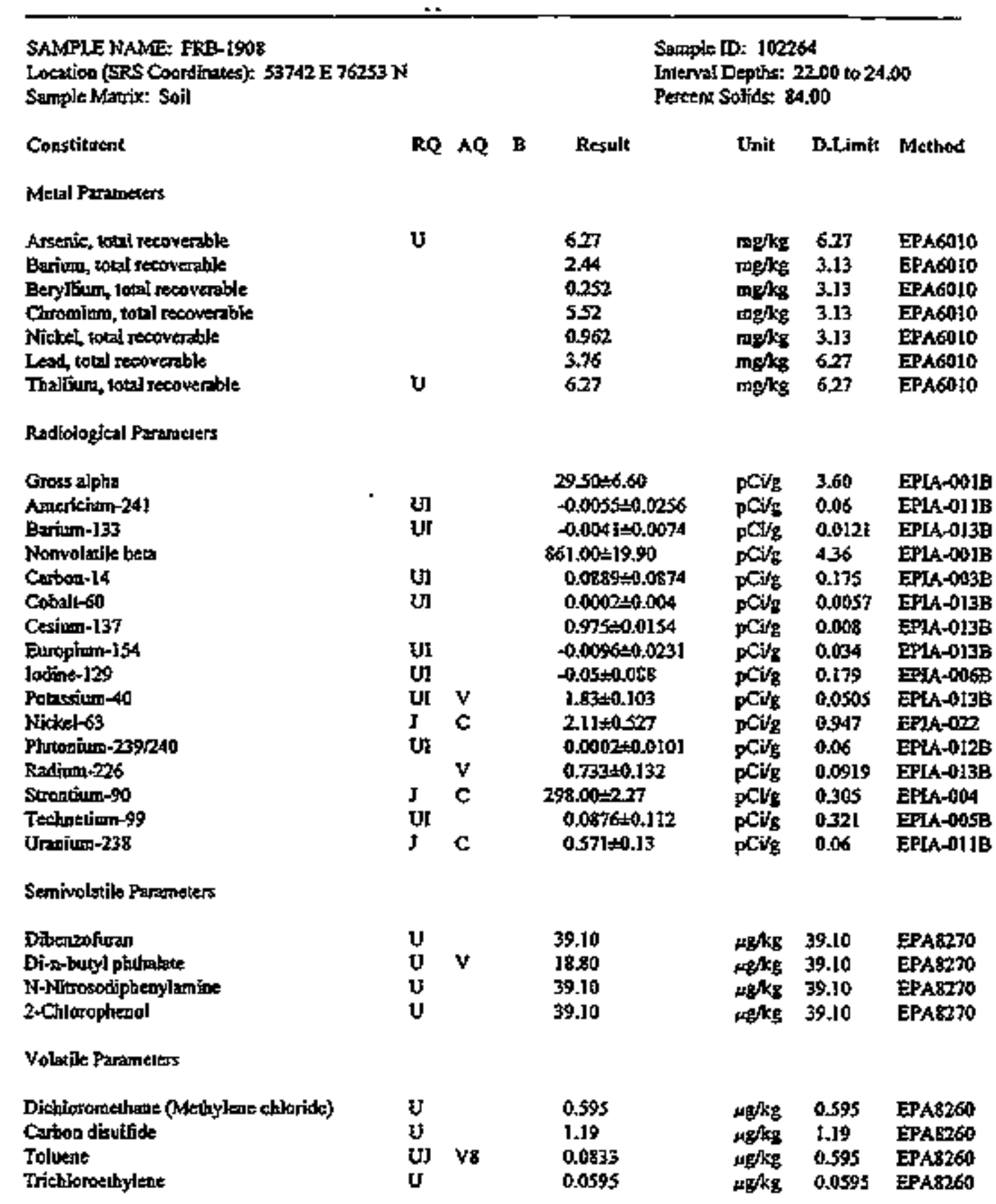

F-Area Relenfion Extip, Phase II 
SAMPLE NAMEE ERB-1909

Lacation (SRS Coordinales) \$9742 E 76253 N

Somple Natrix: Soil

Constituent

Meial Farameiers

Arsenic, total recoverable

Britun, cotal recorerable

Beryllinio, tolel recoverabth

Chrominar, cotnl recoverable

Nicket, rotal recowerable

Lead, total recoverable

Thallium, total recoverable

Rodiological Parameters

Gross alpha

Americium-241

Brrivm-j33

Nonvolarik belo

Carbon-14

Cobnll-60

Certum-137

Europium-154

Iodize-129

Fotessiurn-40

Nivicalo6s

Fuxonium-239240

Radium-226

Smopium-90

Technctium-s9

Unanium-238

Semivolvoile Parameters

Dpeenzotoran

Dirn-butyl phubalete

N-Nimosodinhenylamine

2-Chlorophenot

Valatila Parameters

Dichtoromethane (Methylenc thloride)

Carbon disulfice

Toluene

Triabloroethylene
RQ AQ B Result

Sample 10: 102025

Intervil Depths 24,00 1026.00

Pertintit Solids: 88.00

Unitt D.Lfinit Melhod

$\mathbf{u}$

$$
\begin{aligned}
& 5.98 \\
& t .25 \\
& 0.30 \\
& 3.76 \\
& 0.579 \\
& 5.49 \\
& 4.08
\end{aligned}
$$

mekg $\quad 5.98$

mgkg 2.99

mbikg 299.

meks 299

mgikg 2.99

mekg 598

mgikg 5.98

EPA6010

EPAOOOU

EPA6010

EPA6020

EPAGO10

EPAGOTO

EPA60to

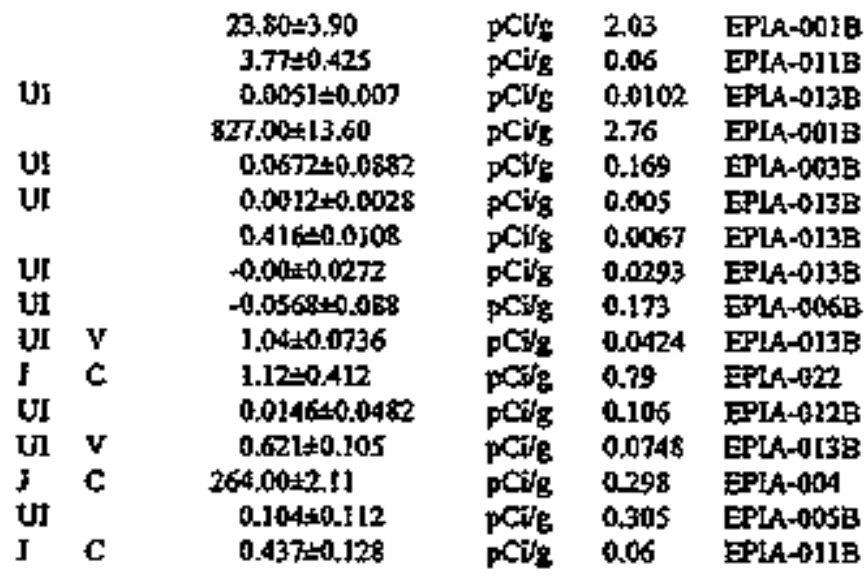

U $\quad 3730$

U $v 54.10$

16.10

Hefsg 37.30

的依 37.30

$\mu \mathrm{g} / \mathrm{kg} \quad 37.30$

$\omega / \mathrm{kg} \quad 37.30$

EPAE2\%

EPA8270

EPA8270

EPA:270

$\begin{array}{lll}U & & 0.568 \\ \text { U } & & 1 .[4 \\ \text { U] } & \text { V8 } & 0.0682 \\ U & & 0.0568\end{array}$

$\mu g / \mathrm{kg}$ 0.56\% EPA\$260

$\mu \mathrm{k} / \mathrm{k}$ 1.t4 EPA3260

$\mu$ OKk 0.568 EPA8260

Mg/k 0,0568 EPA\$260

F-Area Rerention Bssin, Phase 11 
SAMPLENAME FRE-1910

Eacation (SRS Coordinztes): 53742 E 76253 N

Sample Matix: Soil

Constituent

Meial Paramevers

Arsenic, zocal cecoverable

Barium, total recoverabto

Beyllinin, total recoversble

Chronium, toc: resorenable

Nickel, total recoverable

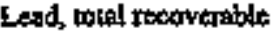

Thallium, tool recoverable

Radiological Parmeners

Gross alpha

Americtum-241

Barium-133

Notvolutile beta

Cribon-14

Cobali-60

Cesium-137

Europium-154

Jodine-129

Potassium-40

Nijicel-63

Photonipum-2296/240

Padfam-226

Strontiver -90

Techlotion-999

Jraniurnw238

Searivolatile Parangaters

Dibenzofiuras

Di-n-buryl phthalate

N-ANitrosodiphenylamise

2-Chlocophenal

Volaule Paramekrș

Dichloromethenc (Mtetrylene chlorade)

Catoon disulfide

Toluents

Triellowoethylene
RQ AO i

U

ur

UI

U!

ut

UI

Ut

UI $\mathrm{V}$

I C

UI

UI $v$

J C

บI

J $ᄃ$

$\begin{array}{lll}\mathbf{U} & & \\ \mathbf{u} & \mathrm{V} & \mathbf{3 6 . 4 0} \\ \mathbf{U} & & 28.00 \\ \mathbf{U} & & 36.40 \\ & & 36.40\end{array}$

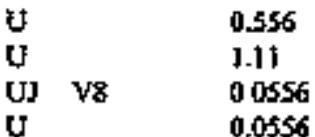

Sample ID: 103266

Interval Depthos: 26.00 to 28.00

Pertent Solits: 90.00

Unit D.Limit Method

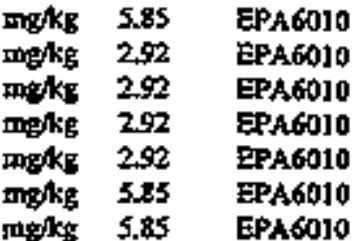

6.10 - 88 pCils 0:292 EPLA+001E

0.01480 .0316 pCis 0.06 EPLA-011B

0.00350 .0055 PCI/S 0.4082 EPIA-OLI3B

$\begin{array}{llll}275.00 \pm 8.12 & \mathrm{PC} \$ \mathrm{~g} & 2.84 & \text { EPIA-O0]B }\end{array}$

$0.0262 \div 0.0869$ pCif 0.166 EPLA-003B

$-0.0004 \pm 0,0028$ pCig 0.4048 EPLA-013B

$0.0062 \div 0.0056 \quad \mathrm{PCI} / \mathrm{g} \quad 0.006$ EPIA-0138

$0.0079=0.0188 \quad \mathrm{pCJ} / \mathrm{g} \quad 0.0278$ EPLA-0LIB

$-0.0039 \pm 0.076 \quad$ PCSS $0.148 \quad$ EPLA-0068

$0.888 \pm 0.0743$ pCis 0.045 EPLA-013B

$\begin{array}{llll}0.99+0389 & \mathrm{pC} / 5 & 0.752 & \text { EPlA-022 }\end{array}$

$0.0192 \pm 0.0281$ pCis 0.06 EPLA-012B

$0.499 \div 0.127 \quad$ pCVg 0.0819 EPIA-013B

$143.00 \pm 2.27$

0.140 .122

PCUL 0.607 EPLA-004

$\mathrm{pCS} / \mathrm{s}$ 0.39 EPLA-005E

PClg 0.06 EPJANDIJ

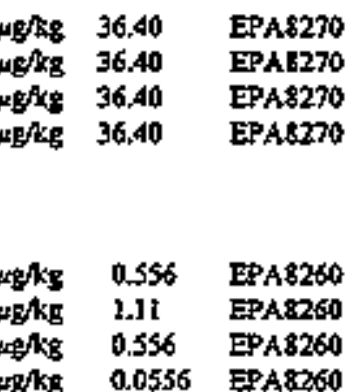

F-Area Retemion Bassn, Prate [I 
SAMPLE NAME: FRB- |91!

Location (SRS Coordinates): 53742 E 76253 W

Sampie Matrix: Sorl

Constituend

Metal Parameters

Assenic, total recoverable

Barium, lokai recoverable

Eeryilitim, tolal recoverable

Chromiam, total recoverable

Nickel, 10tal recowerable

Lead, 10tal recowerable

Thatlium, total recoverable

Radiological Parantecrs

Gross alphe

Americium-241

Earium-133 *

Nopvolatile beta

Carbon-i4

Coboiro 60

C=siom-137

Europium +154

lodine-129

Potessium-40

Nickel63

Phionlun-239/240

Radium-226

Stronliumn-90

Technetiom-99

Urasium-23:

Semivoletile Parameters

Dibenzofurald

Di-n-butyl phthslate

N.Nivosodiphenytamine

2-Chforophenot

Yolatike Parameters

Dichloromethane (Mdelhyiene chioride)

Cabon disukfide

Toluene

Frífblocoethyiene
RQ AQ B Restil

U

I 2.85

v

5.78
0.427
0.0746
1.23
2.89
3.80
5.78

Sarple 10; 102067

Eterval Depthe: 28.00 to 30.00

Percent Solids: 91.00

Unit

D.Limit Method

5.78

melke

mgkg 289. EPA6010

mejts 2.89 EPA6010

mofk 2.69 EPA6010

molke 5.78 EPACO10

megk 5.78 EPA6010

\begin{tabular}{|c|c|c|c|}
\hline $6.34 \div 1$. & pCivg & 0.884 & EPLA-00)B \\
\hline $0.0977 \pm 0.0459$ & pCifs & 0.06 & EPIA-OIIB \\
\hline $0.0036=0.0034$ & pors & 0.0053 & EP(A-O) $3 \mathrm{E}$ \\
\hline $22.90 \div 2.46$ & peiss & 2.47 & EP1 \\
\hline $0.0263 \pm 0.0849$ & $\alpha v_{s}$ & 0.159 & EPLA-003B \\
\hline $0.0012+0.0004$ & $\mathrm{pCis}$ & 0.0036 & EPIA-AI3B \\
\hline $0.0043 \pm 0.003$ & $\mathrm{pCi}_{\mathrm{g}}$ & 0.0044 & EPLADI3L \\
\hline$-0.003 \pm 0.0135$ & pCiss & 0.0195 & EPLA-13B \\
\hline$-0.0297=0.043$ & $\mathrm{pCV} / \mathrm{g}$ & 0.0813 & EPLA-106B \\
\hline $0 . m=0.062$ & pis & 0.0343 & EPLA-013B \\
\hline $1.37 \pm 0.416$ & $\mathrm{PCog}_{\mathrm{g}}$ & 0.775 & EPLA-022 \\
\hline $0.0051=0.0187$ & $\mathrm{PCis}^{-}$ & 0.06 & EPLA $(1) 12$ \\
\hline $0.491=0.0951$ & $\mathrm{pC}$ & 0.0664 & EPLA-013I \\
\hline $38.20 \pm 135$ & $\mathrm{Ci}$ & 0.677 & EPIA-1004 \\
\hline $0.127=0.119$ & $p C$ & 0.311 & EPIA-005 \\
\hline $4 . \$ 42 \geq 0.142$ & $\mathrm{PCi}$ & 0.06 & EPLA-Ot 1 \\
\hline
\end{tabular}

$\begin{array}{lll}u & & 36.60 \\ U & v & 28.50 \\ u & & 36.60 \\ U & & 36.60\end{array}$

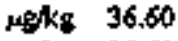

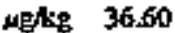

נikg 36.60

- 36 kg 36.60

EPA8230

EPA8270

EPA82 70

EPAS270

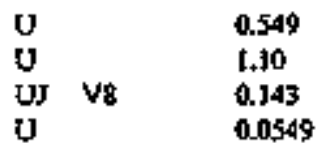

WAkE 0549 EPA8260

LAk 1.10 EPA\$260

toP $\mathrm{kg} \quad 0.0549$ EPA8260 4.k5 0.549 EPA8260

F-Arła Reteodod Basin, Phase II 
SAMPLE NAME: FRE-1912

Locatios (SRS Couroinates): 53742 E $76253 \mathrm{~N}$

Sanple Martix: Soil

Constuttuest

Metal Parameiers

Arsenit, total Jecovenble

Barium, total recoverable

Berylüsm, totei recowerablo

Chrominam, total texarezble

Nilekel, total rocoveatble

Lead, wati rtcoverable

Thilliam, total recoverable

Radioforical Pagramelers

Gross ałpha

Amprition-241

Barium-133

Nonwolatike beta

Carbopo-14

Coball-60

Cesium-137

Exoropium-15d

Iodive-129

Potassiom-40

Nicket-63

Phrodium-239/240

Radinm-226

Stromilinox-90

Terhation-99

Urrinm-238

Semivolatile Parmeters

Dibsarofuras

Di-n-buryl phtipalere

N-Nibosodiphenylamine

2-Chtorophantol

Volatile Parameters

Dithloromathane (Mxtihylenter chloridt)

Cribon disutiods

Toluene

Tricbloroethylene
RO 10 B

U

U

$\mathbf{u}$

5.78

0.585

0.0856

1.12

289

2.52

5.78

$[0.40 \div 2.3]$

$0.014 \pm 0.0241$

$-0.002 \div 0.0049$

$53.80=3.67$

0.032240 .0864

$0.0007 \div 0.0025$

$-0.0013+0.0039$

$0.0138=0,019$

$0.005] \pm 4.034$

$1.02 \pm 0.0734$

$0.74 \pm 0.355$

$-0.008 \pm 0.0992$

$0.773 \leq 0.0962$

$13.30 \div 0.634$

$0.013 \pm 0.105$

$0.863 \pm 0$ 저요

i3i

J C

$\begin{array}{lll}\mathrm{u} & & 36.10 \\ \mathrm{U} & \mathrm{v} & 18.40 \\ \mathrm{u} & & 36.10 \\ \mathrm{u} & & 36.10\end{array}$

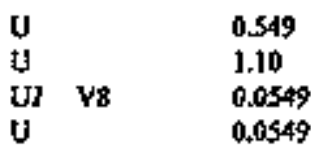

Saraple ID: 100268

Irterval Dephlis: 30.00 th 32.00

Percent Solids: 9t.00

Unit D.Limit Machad

$\begin{array}{lll}m g / k g & 5.78 & \text { EPA6010 } \\ m g / k g & 2.89 & \text { EPA6010 } \\ m g / k g & 2.89 & \text { EPA6010 } \\ m g / k g & 2.89 & \text { EPA6010 } \\ \text { mg/kg } & 2.89 & \text { EPA60I0 } \\ m g / k g & 5.78 & \text { EPA6010 } \\ m g / k g & 5.78 & \text { EPA6010 }\end{array}$

PCSg 2.01 EPIA-OOIB

pCi/s 0.06 EPIA-0I tB

$\mathrm{PCi} / \mathrm{g}$ 0,007 EPLA-013B

DCIS 2.90 EPIA-O01B

PCig 0.162 ELA-003B

DCI/ 0,005 ERLA-013B

pCSg 0.0057 EPLA-0]3B

PCŚ 0.0266 EPIA-013B

PCI/g 0.15\% EFLA-006B

PCW 0.0435 EPTA-013B

PCS 0.724 EPIA-022

pCl/g 0.06 EPlA-012B

PCS/ 0.0695 EPLA-013B

$\mathrm{pCy} / \mathrm{s} 0.381$ EFA-004

QCis $0.279 \quad$ EPMA-005B

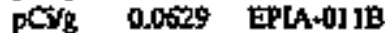

colks 36.10 EPA8270

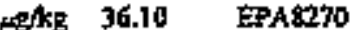

$20 / \mathrm{kB} 36.10$ EPA8270

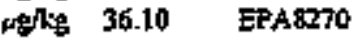

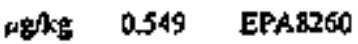

$\mathrm{H} / \mathrm{kg} \quad \mathrm{t} .10$ EPA8260

polk 0.549 EPA8260

$\mu g / \mathrm{kg} 0.0549$ EPA8260

F-Area Retention Basin, Phesse II 
SAMPLE NAME: FRB-1913

Location (SRS Coocdinates): S3742 E 76253 N

Sample Matrix: Solt

Constituent

Metal Paradalents

Arsenic, total recoverable

Barium, total recoverable

Berythfum, notal recoversble

Chrominsm, weal recoverable

Wickel, tocil recowerabls

Lead, 10kal secorterible

Thallium, tokal recoverable

Radiological Parameters

Gross alphs

Americiunn-241

Bariums-133

Nonvolatile totect

Cabon-14

Cobatt-60

Cositim +137

Europiun-154

Jodine-129

Portsiuma 40

Nitket-63

Plutonizm-239/240

Radiun-2026

Strontium-90

Techentiam-99

Uravium-23:

Semivolatile Parameters

Dibereosiuran

DF-p-butyl phthalate

N-2Nitrosodiphenylomine

2-Chlorophenol

Volatile Parameters

Dfchloroneingre (Methylenc atoride)

Cabon disulifide

Tolvene

Trichloroethylape y

$\mathbf{u}$

ป

5.78

0.743

0,0908

0.721

2.89

4.67

5.78

UI

v)

บIJ $x$

UIJ $x$

UD $x$

UI $v$

J C

UJ $\mathrm{CX}$

UI $V$

J CI

UI

J C

$11.30=3.27$

$0.965 \pm 0.103$

$0.0042 \div 0.0041$

$66.50 \pm 5.73$

$0.0549=0.0876$

$-0.0004 \pm 0.0026$

$0.0088=0.0044$

$0.0086 \leq 0.0133$

$0.027 \div 0.046$

$0.898 \div 0.0792$

$0.911 \pm 0.39$

0.000280 .0115

$0.584 \pm 0.105$

L $\quad 24.90=0.83$

$0.0747 \pm 0.128$

$0.363 \pm 0,11$
Sruple ID: 102269

Interval Depths: 33,00 10 34,00

Pertent Solvits: 91,00

Unit D.Einst Method

mpifk $\quad 5.78$

mo/ks 2.89

289

Inefie 289

mDKa: 2,89

$\mathrm{mgkg} \quad 5.78$

JL/kg $\quad 5.78$

EPAG0I0

EPASDI0

EPA6010

EPA6010

EPA6010

EPA6010

EPAG0IO

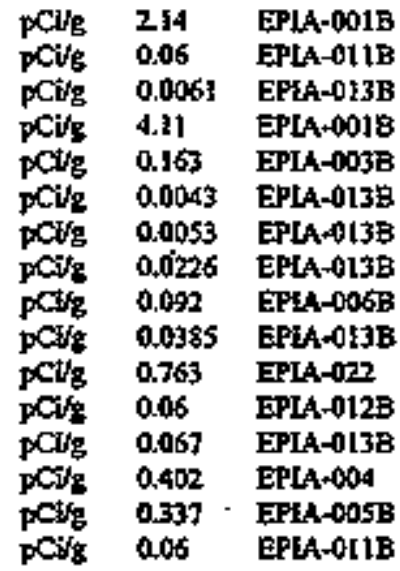

apHer 36.40 EPA 2270

$\mu g / k 8$ 36.40 EPA\$870

$\mu$ 就 $\mathrm{kg}$ 36.40 EPA8270

$\mu / \mathrm{kg}$ 36.40 EPA8270

U $\quad 36.40$

U $\quad 36.40$

0.549

1.10

$\begin{array}{lll}\text { UJ V8 } & 0.0549 \\ \text { U } & & 0.0549\end{array}$

\begin{tabular}{lll} 
U & & 0.549 \\
U & & 1.10 \\
UJ & V8 & 0.0549 \\
U & & \\
\hline
\end{tabular}

$\begin{array}{lll}\mu g / k g & 0.549 & \text { EPA8260 } \\ \mu \mathrm{g} / \mathrm{kg} & 1.10 & \text { EPA8260 } \\ \mu \mathrm{g} / \mathrm{kg} & 0.549 & \text { EPA8260 }\end{array}$

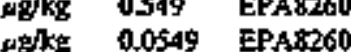

F-Area Relenrion Bastw, Phase It 
This Page Intentionally Left Blamk 


\begin{abstract}
APPENDIX C
Environmental Measurement-While-Drilling-Gamma Ray Spectrometer

Data Collection Procedure
\end{abstract}

C-1 
This Page Intentionally Left Blank

$\mathrm{C}-2$ 


\section{EMWD-GRS Data Collection Procedures}

\subsection{Check System Operation on Site}

Check for full aperation of tool and computer interface prior to attackment

of downhole tool to drill string or mounting uphois components on drill rig.

\subsection{Mount System and Verify System Functionality}

A. Downhole battery voltage $>22 \mathrm{~V}$.

B. Temperature measurement.

C. Collect normal background spectrım at ơill site.

D. System lock reading - 0eb90 Hex.

\subsection{Start Recording Data to Disk and Commence Drilling}

A. Continuous recording of data while drilling as follows:

1. After each 3 drill rod sagments, stop recording, close data fille and name (i.e., RB_F01.PCM, RB_F04.PCM, otc.)

2. Restart recording under a new data file marne, start drilling the next tiree rod segments.

3. Repeat steps 1 and 2 untit drilling is completed.

B. At or near sampling location, stop drilling and record spectrum for a predetermined amount of time. 
This Page Intentionally Left Blank

C-4 


\section{APPENDIX D \\ Environmental Measurement-While-Drilling-Gamma Ray Spectrometer Spectral Gamma Calibration Procedures}

The draft procedures for calfbrating the EMWD-GRS systems gamma ray spectrometer are outlined in this Appendtix. Please note:

1. Laboratory calibration procedures are outlined in the draft procedures.

2. Field calibration procedures: Currently, there are no field calibration procedures for the environmental data collection. The calibration for this demonstrafion will be a function of the WSRC extended C\$-137 source model.

Dw1 
This Page Iatentionalyy Left Blank

D.2 


\section{EMWD Spectral Gamma Calibration and Field Measurement}

\section{Introduction}

There are two main elements for converting spectral gamma energy readings into a indication of soll contamination levels. First is the linear correlation of gamma energy Vs channel location. In general this correlation can be deternined in the lab using known source material emitting gamma particles at differing energy levels. Second is the calibration of gamma flux density Vs contamination levels. This second process is not . directly determined by laboratory standards. In fast this second step is undier investagation at many DOE waste sites.

In this report a calibration process is looked at for the spectral gamma Nal detector used in the environmental measurement while driting system (EMWD). A quick look at linear channel calloration is given, using actual EMWD laboratory data. To better understand the unfolding process for calculating redionuclides, a short explaination for unfolding naturally occuring radionclides for uranium exploration is given. This process is also used

to gage the preformance of newhy developed spectral systens for eovironmental work. Following the unfolding process for matural radiation will be a look at actual spectral logging data from a waste site and an unfolding method for cesium and cobalt.

The final goal of this work is to justify and document reasoning for taling a simplier approach concentrating on cesium detection.

\section{Gamma Energy Vs Channel Locatiou}

This function very closely matches a straight line with a zero intercept, measured gamma energy $=a$ * (Channel Number) + b. The NaI crystal sensor is exposed to differing radio maclide emitting gamma particles of differing energy leveis. Exposure is contimued until peaks appear in the spectrum at count levels assuring accurate peak channel measurement, normally $>100$ counts or X10 background. Below are the laboratory measured values for the given sources.

\section{Table 1: Linear Calibration Results}

$\begin{array}{lccc}\text { Sorrce Element } & \begin{array}{c}\text { Peak Energy } \\ (\mathrm{MeV})\end{array} & \begin{array}{c}\text { Penk Channel } \\ \text { Number }\end{array} & \begin{array}{c}\text { \% Difference } \\ \text { From Calc. }\end{array} \\ \text { Cs } 137 & 0.662 & 92 & 1.1 \\ \text { Co } 60 & 1.173,1.332 & 163,186 & 0.7,0 \\ \text { Mn } 54 & 0.835 & 115 & 1.7 \\ \text { Na } 22 & 0.511,1.275 & 74,178 & 2.9,0\end{array}$


The resulting linear regression for energy $V s$ channel number is: $Y \mathrm{MeV}=7.18 \times 10^{-3}$

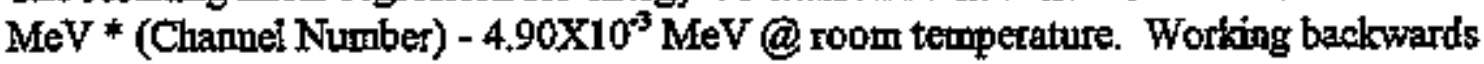
using the given chamel munber and the known energy gamma the percent deference was caiculated. The correlation coefficient of Table 1 values is 0.9996 . The linear response of a NaI detector is very good. However, a number of factors can cause the slope "ax" to change while drilling, primarily temperature, high voltage drift, and photon-multiplier tube aging. Controlling these parameters is critical to proper measurement.

\section{Flux Density Vs Contamination Levels}

Gamma counts rate is a relative measure of gamma flux, dependent on many factors as detector size, housings, ect. This flux is proportional to the amount of radioactive material in the soil. Thus, the measured flux is converted to $\mathrm{pCi} / \mathrm{g}$ by calibration coefficients derived from calibration models. These models have known amounts of source material distributed in a large enough volume to appear infinitely large to traveling gamma rays, about a two to four foot radius about the sensor.

However, soil conditions infinitely vary for moister content and physical make up. Moister and soll types influence the measured gamina flux. Limitations in calibration for flux density Vs contarnination levels in soil tesult in an assumption that all soil conditions are consisteat with the calibration models.

The most commonly used calibration models are maintained for DOE's Grand Junction Projects Office in Grand Junction Co. by contract with Rust Geotech Inc. These models were brilt to calibrate instrumentation used for uranium exploration. As such these models contain toree naturally occurring elements, $\mathrm{K}-40$, Ra-226, and Th-232, (KUT). Because these models are well characterized and documented they are used to set a baseline accuracy for all subterranean gamma instrumentation Stromswold (1981) uses gamma count windows centered about energy peaks of the three naturals which unfold from highest energy to lowest. Table 2 shows his suggested windows.

\section{Table 2 Spectral Energy Windows for Unfolding KUT}

Element
Potassium (K-40)
Uranium (Ra-226)
Thorium (Th-232)

Unique Gamroa Ray (MeV)
1.46
$1.76 \& 2.20$
2.61

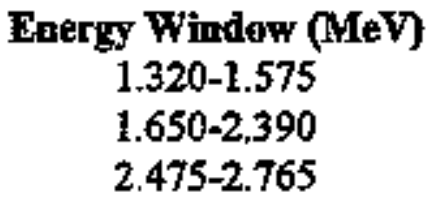

In working with subterranean garmon there is a problem of higher energy gamma rays being counted in lower chamels, down scattering. By choosing the Thorium window about the $2.6 \mathrm{IMeV}$ gamma, Thorium can be solved for because potassium and uranium don't have any gamma rays higher than $2.39 \mathrm{MeV}$. Once thorium is known then the solution for urarium can be found because potassium is below the 1.65MeV window used for uranizm. This process is called unfolding. The Grand Junction B models are well suited for this unfolding process. The B model concentrations listed in Table 3 below. 


\section{Table 3. Grand Junction B-Model Concentrations}

$\begin{array}{lccc}\text { Model } & \begin{array}{c}\text { Concentration Th } \\ (\mathbf{p C i} / g)\end{array} & \begin{array}{c}\text { Concentration Ra } \\ (\mathrm{pCi} / \mathrm{g})\end{array} & \begin{array}{c}\text { Concentration K } \\ (\mathbf{p C i} / \mathrm{g})\end{array} \\ \text { BT Upper } & 58.78 \pm 1.53 & 10.46 \pm 0.51 & 10.13 \pm 1.34 \\ \text { BU Upper } & 0.65 \pm 0.06 & 194.59 \pm 5.94 & 10.63 \pm 1.00 \\ \text { BK Lower } & 0.10 \pm 0.02 & 1.03 \pm 1.67 & 54.00 \pm 1.67\end{array}$

By placing the spectrometer into each of the three models, subtracting electrical noise, and counting garmma for each of the three windows in Table 2, a rate matrix $R$ is produced. Matrix $R$ is guaranteed to be nonsingular because of the window selection process assures an upper triangular form. Using the concentrations of Table 3 a set of coefficients relating window count rates to concentrations ( $\mathrm{pCl} / \mathrm{g}$ ) can be solved for using Eql. An important note on counting periods; The statistical nature of gamma counting requires long enough counting periods to gain a meaning full count rate. The standard deviation of the gamma count is equal to its square root, i.e. 100 counts has a 10count sdv.

\section{$A \quad=\mathrm{CR}^{* 1}$ Eql.}

$A$ is a $3 X 3$ Matrix of Calibration Coefficients

$R$ is a 3X3 Matrix of Count Rate reading for each of the three windows

$\mathcal{C}$ is a $3 X 3$ Matrix of Known model concentrations from Table 3

Once $A$ is known then the system is tested against a forth model (BM) which is a mix of all three elements. A properly calibrated spectrometer then solves for concentration leve's for KUT using equation $\mathrm{Eq} 2$.

$$
C=A R \quad E q 2 .
$$

Equation 2 is uset to convert garnma flux rates to density measurements in $\mathrm{pCl} / \mathrm{g}$ as the system is drilling or logging. There are a number of aciditional considerations to the process which should be addressed. First, the linear calibratioì relating gamma energy peaks to channel mumbers in the spectrum is used for setting the KUT windows of Table 2. Anything which alters this calioration effects the calculated concentration levels. The measure of the gamma rate is dependent on concentration levels but also the MCA conversion rate. Low power MCAs normally employ slow conversion methods inereasing dead time (DT). Where DT and $R$ are both in units of seconds, $E q 3$ below is used compensate for a slow MCA

$$
R^{R}=R^{*} \text { Isec/(Isec - DT) Eq3. }
$$

$D T$ is a function of $M C A$ total coumts and conversion time

$R$ ' is a new $M C A$ compensated rate matrix 
In the general solution of converting garsina coumt rates to KUT soil concentrations, a basic assumption was mare; Only raturally cocuring garnma sources are found in the soil. The man-made rad waste creates a new set of gamma emmitters in contaminated soils.

In the case of Cesium (Cs-137), its' gamma ray is at $0,66 \mathrm{MeV}$. Using this unfolding process Cesium would be unfolded after potassium. Too follow this logic, every radioactive element distributed within the soil must be accounted for in the unfolding process. The dominate waste radionuclides generally found in the soils at Hanford and Savannah River are Cesium-137, Europium-154, Europium-152, and Cobalt-60. In a Westinghouse Savannah River 1994 report on H-Area retention basin tist maximum concentrations as shown in Table 4. Table 4 is by no means a complete list of man-made waste, rad or otherwise.

Table 4. Example of found Radionaclides at a Waste Site
$\begin{array}{cc} & \\ \text { Radionuclides } & \text { Max Concentration, pCig } \\ \text { Cesium-137 } & 33000 \\ \text { Europium-152 } & 47 \\ \text { Europium-154 } & 33 \\ \text { Cobalt-60 } & 1.8\end{array}$

Figure 1 is log data taken with a HPGe detector used at Hanford, (C.J. Koizumi, 1993). There are two important attributes demonstrated by this data. First, the total coumt is a good indicator of waste radionuclides in the soil. Second, cesium waste maybe independent of other tadionuclides.

A complete gamme spectrum is shown in Figure 2. This spectrum was taken at $16.8 \mathrm{~m}$ depth in the log run shown in Figure 1. Here the spectrima is scaled out to $2.8 \mathrm{MeV}$. By scaling out so high the thorium peak at $2.6 \mathrm{IMeV}$ can be monitored for changing backgrounds. The measured concentrations for this spectnum at as follows: $3 \mathrm{pCi} / \mathrm{g}$ of Co- $60,29 \mathrm{pCi} / \mathrm{g}$ of Eu-154 and $8 \mathrm{pCi} / \mathrm{g}$ of K-40. The vast majority of spectral activity is below the K-40 peak at $1.46 \mathrm{MeV}$.

Looling e gain at Figure 2, the down scattering of higher energy gamma into the $0.66 \mathrm{MeV}$ energy chanel is a concern. Because of the low energy Cs-137 gamma vitually all backgroung and other man-made rad waste interferes with the cesilum measurement. 


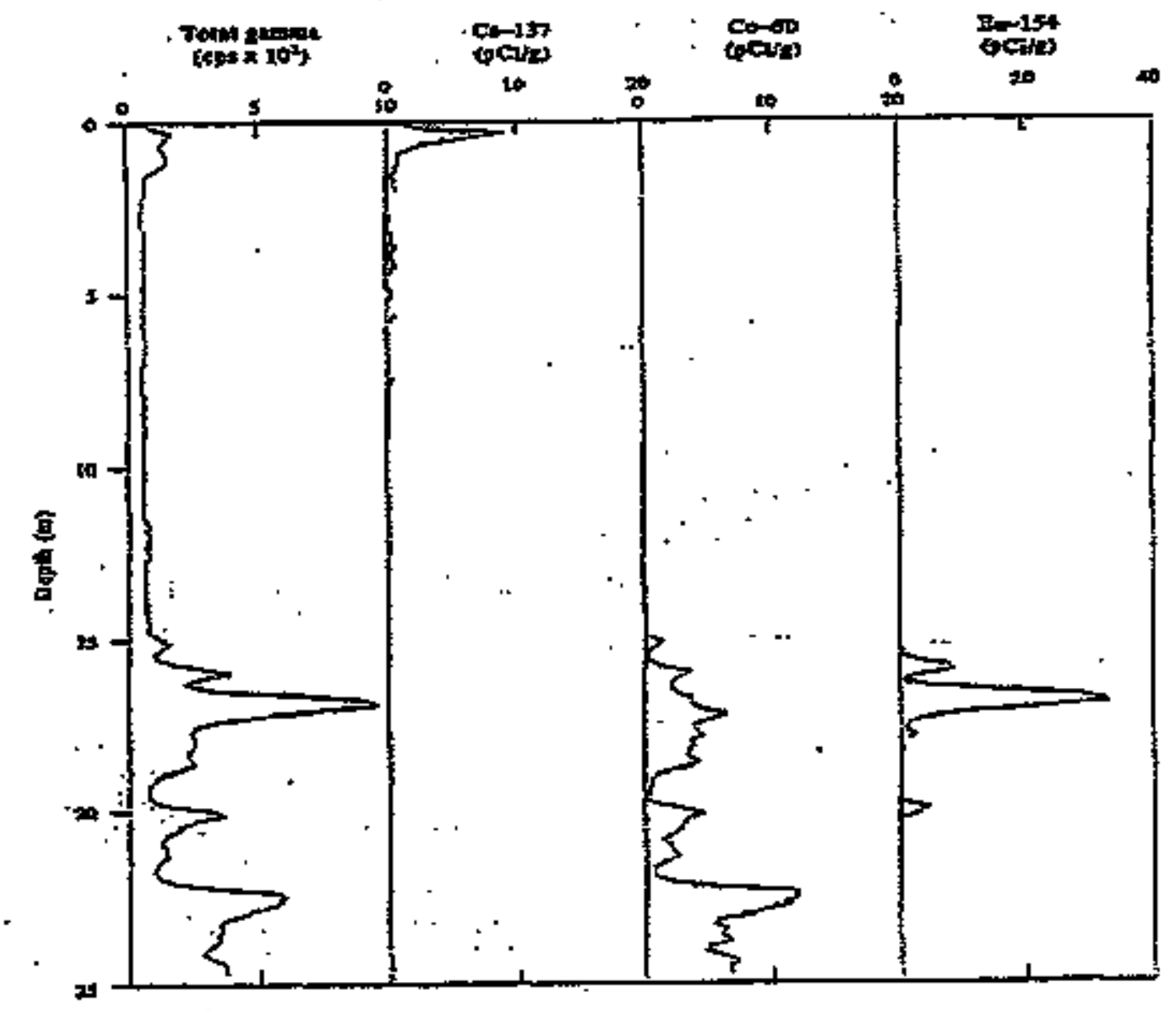

Figure 1. Spectral log near Hanford waste tank, (C.J. Koizumi, 1993)

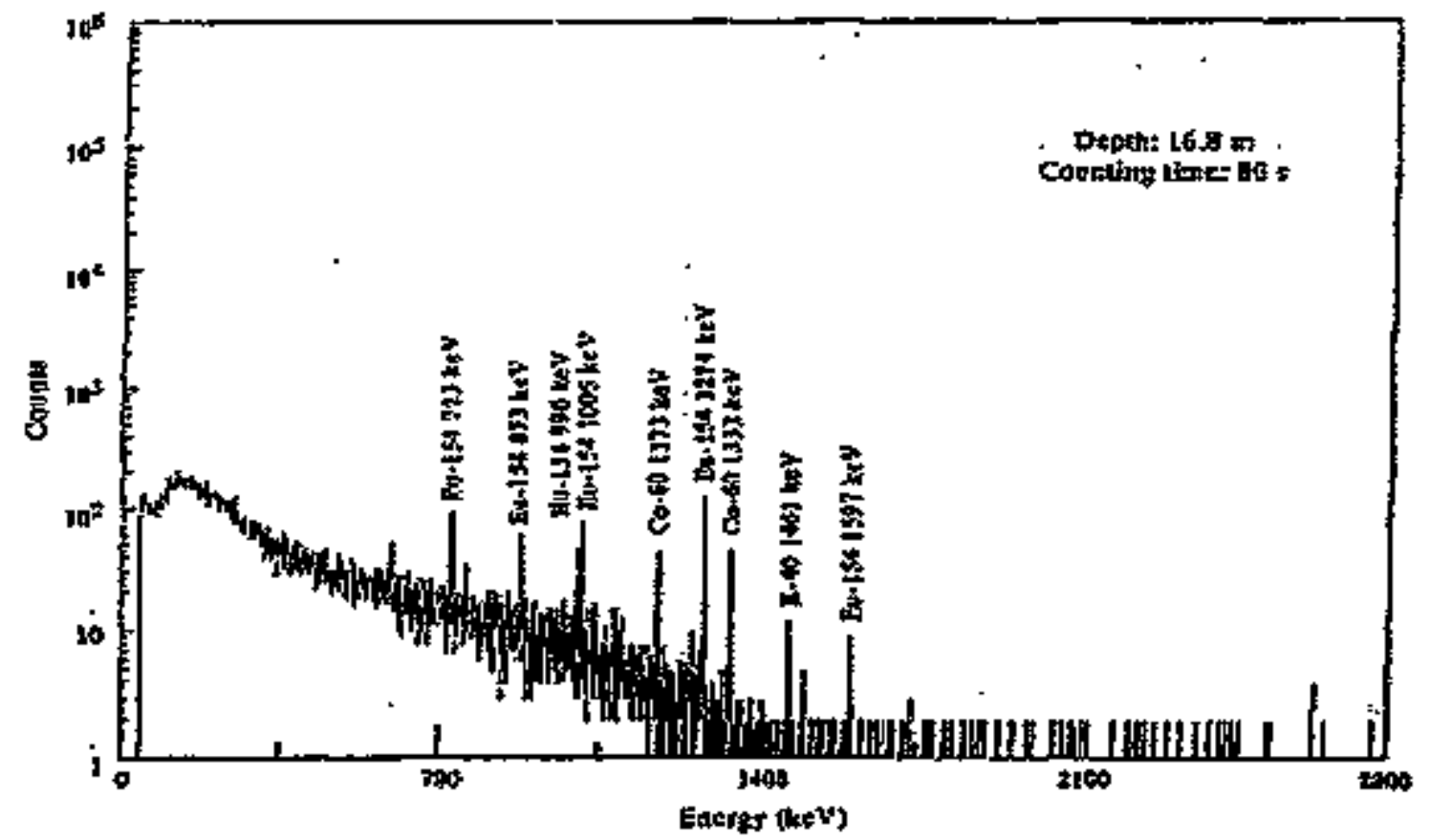

Figure 2. An example of a complete gamma spectrum taken for the log in Figtre 1. (C.J. Koizuni, 1993)

D-7 


\section{Unfolding Co and Cs From Background, An Example}

Unfolding the three raturals along with cesium and cobalt (Randall and Stromswold, 1995) used windows 1.105 to $1.420 \mathrm{MeV}$ for cobalt and 0.590 to $0.715 \mathrm{MeV}$ for cesium. Lumping the background $\mathrm{T} h$ and $\mathrm{U}$ counts as a single constant term, the $\mathrm{Cs}$ and $\mathrm{Co}$ unfolding formulas are shown below.

$$
\begin{aligned}
& C_{c o}=\mathrm{aR}_{\mathrm{cos}}-\mathrm{bR}_{\mathrm{x}}-\mathrm{CR}_{\mathrm{cs}}-\mathrm{BKG}_{\mathrm{co}} \text { Eq4. }
\end{aligned}
$$

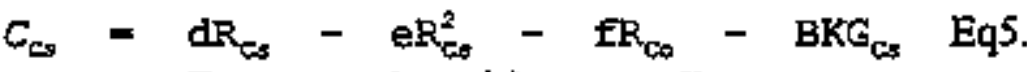

$$
\begin{aligned}
& \text { Terms ' } a \text { '-'f' are inigue coefficients. } \\
& B K G \text { is the constint backgrond subtraction of each element. } \\
& \text { In all cases } B K G_{C s}>B K G_{C o} \text {. }
\end{aligned}
$$

Both equations 4 and 5 use the K40 rates directly. This is done because the cobalt upper gamma is very near that of potassim. The NaI detector rosolution will overlap gamma counts. In Eq5 has a cobalt count rate term for calculation of cesium. Often cesium and cobalt are found together and the down scattering of the higher energy cobalt is a significamt. Eq5 incorporates a squared term for pile up correction at very high count rates.

\section{Suggested Approaches For EMWD}

The EMWD MCA is a 256 channet multi-channel analyzer. The NaI crystal is (at present) a four by one inch cylinder. Complete spectrums are transmitted to the surface every 30 seconds. Spectrums are not being taken while data is being transmitted. The actual sample period is -20 seconds. Spectrums can be summed at the surace to longer sample periods.

The main focus of the EMWD system is to detect and measure cesium contamination levels white drilling. There are no cesinm waste models for calibration of spectral gamma logging systems. Even if such a noodel existed there are too many types of mixed radionuclides at each DOE site for any NaI system to accurateily unfold. Two methods are suggested for calibrating a system to unfold Cs-137 from natural background spectrums. In both cases, total gamma counts will be used to detect increased levels of man-made waste. The total cotat might also help detect when count rates are increased by manmade waste other than Cs-137 by the simple relationstip in Eq6.

$$
\begin{gathered}
T C-\mathrm{aR}_{\mathrm{Cs}}-\mathrm{bR}_{\mathrm{x}}-\mathrm{BKG}_{\mathrm{TC}}=0 \mathrm{EqG} \\
T C=\text { total coumts } \\
B K G_{T C} \text { taken from reading is a clean area. } \\
a \& b \text { coefficients derived from field testing. }
\end{gathered}
$$




\section{Calibration Method I}

This method would treat the spectrum readings in the same fashion as calibrating any spectral gamma logging system as addressed earlier in this report.

Set the linear range to $2.80 \mathrm{MeV}$, fill scale. Choose windows for all three naturgls phus Cs-137. Eq1 is now composed of $4 \mathrm{X} 4$ matrixes. B-models can be used where the model concentration of Cs-137 is assumed zero. To solve for matrix A a forth model of known concentration of $\mathrm{Cs}-137$ must be used. This $\mathrm{Cs}-137$ model may actually be a characterized well as logged in Figure I at a waste site. This approach is heavily dependent on the quality of the $\mathrm{C}$ - 137 model. The matrix inversion simultaneous solution of linear equations produces a least squares fit to given data. The solution maybe sensitive to slight changes in concentration levels, non-robust. This problem is compounded by the lack of a properly configured mixed model to help test the solution.

\section{Calibration Method 2}

The energy range will be low, upper end limited at $1.6 \mathrm{MeV}$. This is done to utilize system sensitivity about the range of interest, see Figure 2. Gamma rays above this threshold are counted as a total and stored in channet 255 . By monitoring this channel normal thorium and uranium background levels can be monitored. These background levels will be characterized at the site by drilling a short bore outside of the contasninated area. Along with channel 255, the potassium and cesium windows will also be characterized for background down scattering. Using the B-model, the cesium window can be characterized for potassiem down scattering.

$$
\mathrm{C}_{\mathrm{s}}=\mathrm{a} \mathrm{R}_{\mathrm{cs}}-\mathrm{bR}_{\mathrm{K}}-\mathrm{BKG}_{\mathrm{Cs}} \quad \mathbf{E q} 7
$$

Several cesium dominated wells of differing levels will be required to curve fit systern

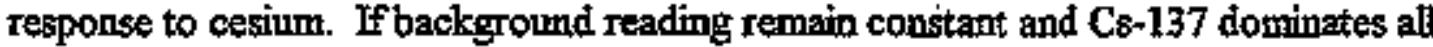
other types of man-made waste then the linear relationship should be well bounded.

\section{Conclusion}

The EMWD spectrometer is capable of linear calibration of gamma energy peaks at room temperature. The logging indistry in cooperation with DOE has developed spectral gamma calibration methods and facilities. These method and facilities are not sufficient to fully calibrate spectral ganoma systems for subterranean measurement of man-made mixed waste.

Actual logging data taken of rad waste by a HPGe system points to the complexity of the problem. For the EMWD system using a NaI detector there is no recogsized solution for callibration or unfolding spectrums in man-made raut waste sites with urknown radiomudide.

Two methods were looked for calibration and unfolding. One method expands the accepted method usei for spectral gamma logging tool calibration used in uranium exploration wells. The second method assumes a fixed background and atterapts to equate a linear relationship between gamma count rates in cesium directly. Both methods 
or some combination of approaches needs to be tested before release for site characterization.

'R. Leim, D.C. George, B.N. Kty, I. Knigith, and W.D. Steele, Jume 1994, Third Edfition, Field Catihation Facilities for Environmiental Measrmeminnt of Racium, Thorium, and Potassium, technical Meagrirements Center Grand Janction Projects Office 


\section{APPENDIX E}

Site Specifíc Health and Safety Plan for the Environmental Measurement-WhileDrilling-Gamma Ray Spectrometer System Demonstration at the SRS F-Area Retention Basin 
This Page Intentionally Left Blank

E-2 
$4 / 2 z / 94$

Document Prepare

thetis \&. Doszalls 11/20/94

SITE SPECIFIC HEALTH

AND SAFETY PLAN

FOR THE

F-AREA RETENTION BASIN (281-3F)

PHASE II RI INVESTIGATION

WORKING COPY

VERIFIER TO BE THE LATEST REVISION

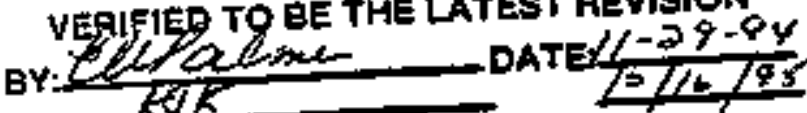

MUST BE VERIFIED PRIOR TO EACH USE

Q-SHP-F-00003

REVISION 01

NOVEMBER 1994

E-3 
Pitre U RI Chenacterizalion

(This page intentionally left blank.) 
Silo Splenetic Hitesto and Safety Pan for the

Fora Retention Basin (281-3F)

Phase if RI Cheradtetization
Q-STIP-F-00003, Row, 01

November 1994

Page ur of

SITE SPECIFIC HEALTH AND SAFETY PLAN

FOR TEE

F-AREA RETENTION BASIN (281-3F)

PHASE I RI INVESTIGATION

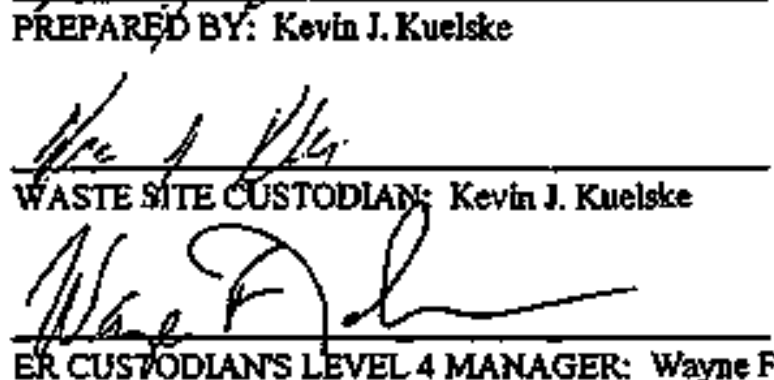

ER CIS VOLANS LEVEL 4 MANAGER: Wayne F, Johnson

Rubleeserfor C Lark RADIOLOGICAL CONIROL and HEALTH PHYSICS MANAGER $\frac{11 / 2 / C H}{\text { DATE }}$

$\frac{11 / 22 / 4}{\text { DATE }}$

$\frac{11 / 28 / 54}{\text { DATE }}$

$\frac{41-28-94}{\text { DATE }}$

$\frac{11 / 25 k 4}{\text { DATE }}$

$\frac{1 / 22 / 94}{\text { DATE }}$

$\frac{11 / 28 / 94}{\mathrm{DAtE}^{2}}$

$\frac{11 / 2 G / S 4}{\text { DATE }}$

$\underset{\text { DATE }}{1 / 2 q / \sigma q}$

E-5 
(This page intentionally left blank.) 
Sik Specific flealih end Sifty Hat for the

F-Ara Retention Bation (281-37)

Phise II RI Chusactistition

\section{HST OF REVISIONS}

$\begin{array}{ll}\text { Revision Nomber } & \text { Revision Date } \\ \text { o } & \text { Jume } 1994 \\ 1 & \text { November } 1994\end{array}$

Bapes Affected

All

$x_{,} 1-10,12,13,18,19,24$ 
Fonra Ration Buin (201-5F)

(This pege intentionally beft blank.) 
TABLE OF CONTENTS

Buge

1.0 INTRODUCTION

2.0 SITE HISTORY AND PROIECT DESCRIPTION

3.0 SITE ORGANIZATION AND RESPONSHBIITIES

4.0 HAZARD AND RJSK ANALYSIS

5.0 SAFETY AND HEALTH TRAINING

12

6.0 PERSONAL PROTECTIVE EQUIPMENT AND PROCEDURES

7.0 MEDICAL SURVEILLANCE

8.0 HEALTH AND SAFETY MONTTORING

9.0 SITE CONTROL

I0.0 DECONTAMINATION

11.0 EMERGENCY RESPONSE PLANS

12.0 CONFINED SPACE ENTRY

13.0 SPLL CONTROL

14.0 RECORD KEEPRNG

15.0 REFERENCES 
Sie Specifit Hectith and Sifety Fin fint the

F-Ava Retionion Buin (281-31)

Plosen II RI Chy netertation
QStPP-F-00063, Ftev. Of

Nowntibet 1994

Pats vin of $x$

(This pege intertionally teft blank.)

E-10 


\section{List of Figares}

Page

Figure 1. Soil, Sunface WatcriSodiment, and Geotectioical Sampling Locations

Figure 2. Soil and Geotechnical Sampling Lacations Along the Process Line

Figure 3. Schenatic of Work Zones

Figure 4. Schematic for Personnel / Equiputent Control

List of Tables

Table 1.

F-Area Retention Basin Expostre Pathways

Tabie 2.

Training Requirennets for Waste Sire Workers

Tabic 3.

ERD Phone Listing 
Phese II If Cbaraterization

(This page intentionally left blank.)

E-12 
Ltst of Acronyms

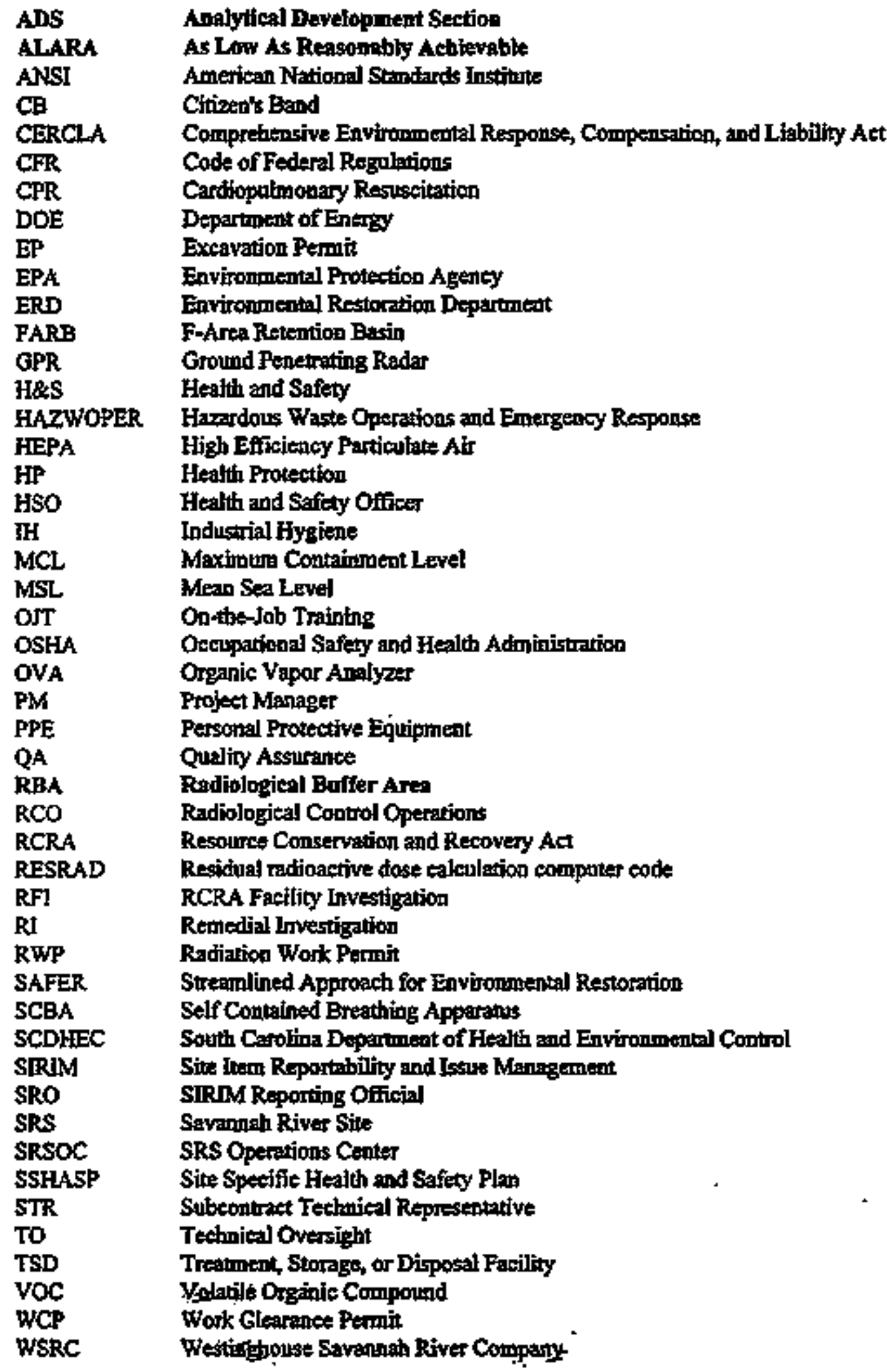


(This page intentionalty left biank.)

E-14

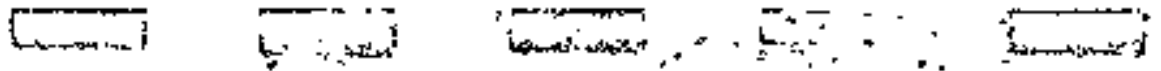


This document describes the safety; health, and emergency response recuitements for investiontion aetivities at the existing F-Area Retention Basith, 281-3f (FARB). The Sire Specific Health and Safery Ptan (SSHASP) contains infornation concerning personnel rspponsibilities, waste unit characterization, environmental montoring, personal protective equjpraent, and required safety and health procedures. In adfition to the requirements outined in this SSHASP, all work will be performed in accordance with WSRC 4Q (Industrial Hypiene Manual), WSRC 8Q (Employee Safety Manuai), WSRC 5Q (Rodiologital Control), WSRC 200 (Health and Safaty Manual for Hazardous Waste Operations), WSRC 3Q5 (Hytrogeologie Date Collection), and ths WSRC RFI/RI Program Ptan (latest revision). In addition to the requirements of this SSHASP, subeontractors and WSRC einployees involved with stbeobtractor atifivities are subject to the safety requirements of the subenatractor or WSRC, depending upon which requirements are more stringent.

\section{SITE HISTORY AND RROJECT DESCRIPTION}

\section{Unit Deseription}

The F-Area Retention Basin (281-3F) wats used from 1955 to 1973. This open, uplined basio provided temporary emergency storage for potentially contaminated cooling water frorl the chemiesl separations process. Whet radioactivity was encountered in the cooling water, finnediate action was baken to diver the water from surfact drainage streans to the retention bosin. The cooling water couls potentially be consmoinated if chenical processing makerials. leaked into it. Daring the holding period, some quantily of water seeped into the ground If the radjoaceivity was above strean release limits, the waste water was processed by deionization to roxuce contanination and permit release. The exact quantities of water disposed in the retention basin is unknown. The volume and curie content of the water in the intembithent discharge varied. only trace quantitites of chemicals are believed to have beas discharged to the basin.

The unlined retention basin stopped receiving effluent in 1973 when it was replaxed by a new, lined retention bastin (281-35). After a characterization study, $0.6 \mathrm{~m}$ of sediment was removed from the floot of the F-Area Retention Basin in 1978-79. The basin was backilled with dit and covered with grats.

\subsection{Projest Description}

The Phase II RI Invesilgation activities ot the F-Ares Retention Basin (FARB) will involve collection of stream surface water and stidment is well as soll samples from within apd surrounding the bassin and associated process sewer line. The activities to be performed are outilined to the Pbase 1 , Rev 1 RI Work Pien

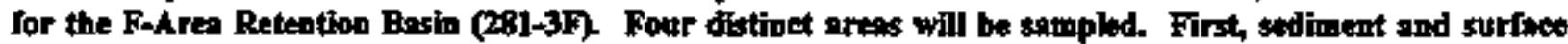

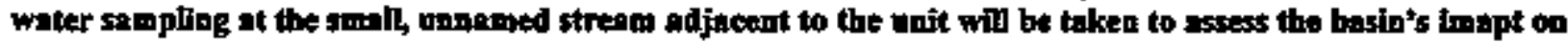
the surrounding wetlondi. This activity will be performed in a radiobgically clean area and will aot reguire a radiolagien bafier ares (RBA). Secobd, soil samples will be collected from four lacetion whth the former basin to a depth not to erowed the grouad water table. Third, soil gompla will be ellected from efight

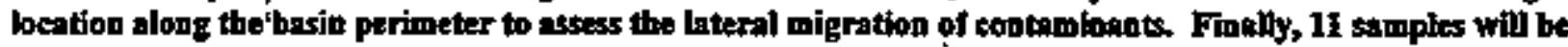

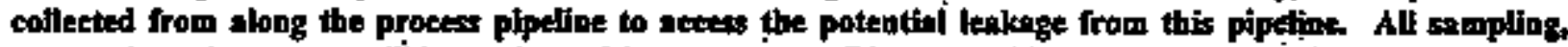
except along thestream, wil be performed in a tewporary RBA end will require contioutias: RCO Coverage

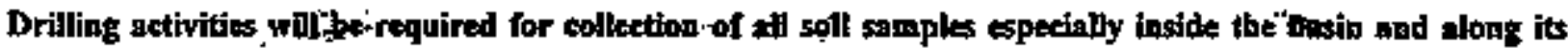
perimeter. Along the process sewer bine, which is nostly-inside F-Area, hand augers will be uzed down to 10 Ieet to ensure no uaderground atilities are encoubtered. All samples collected will be sereen by efther RCO (if "blue tagged") or by ADS prior to shipment off site for acalysis. Proposed sampling beation ten belsteto in Figores 1 and 2. 


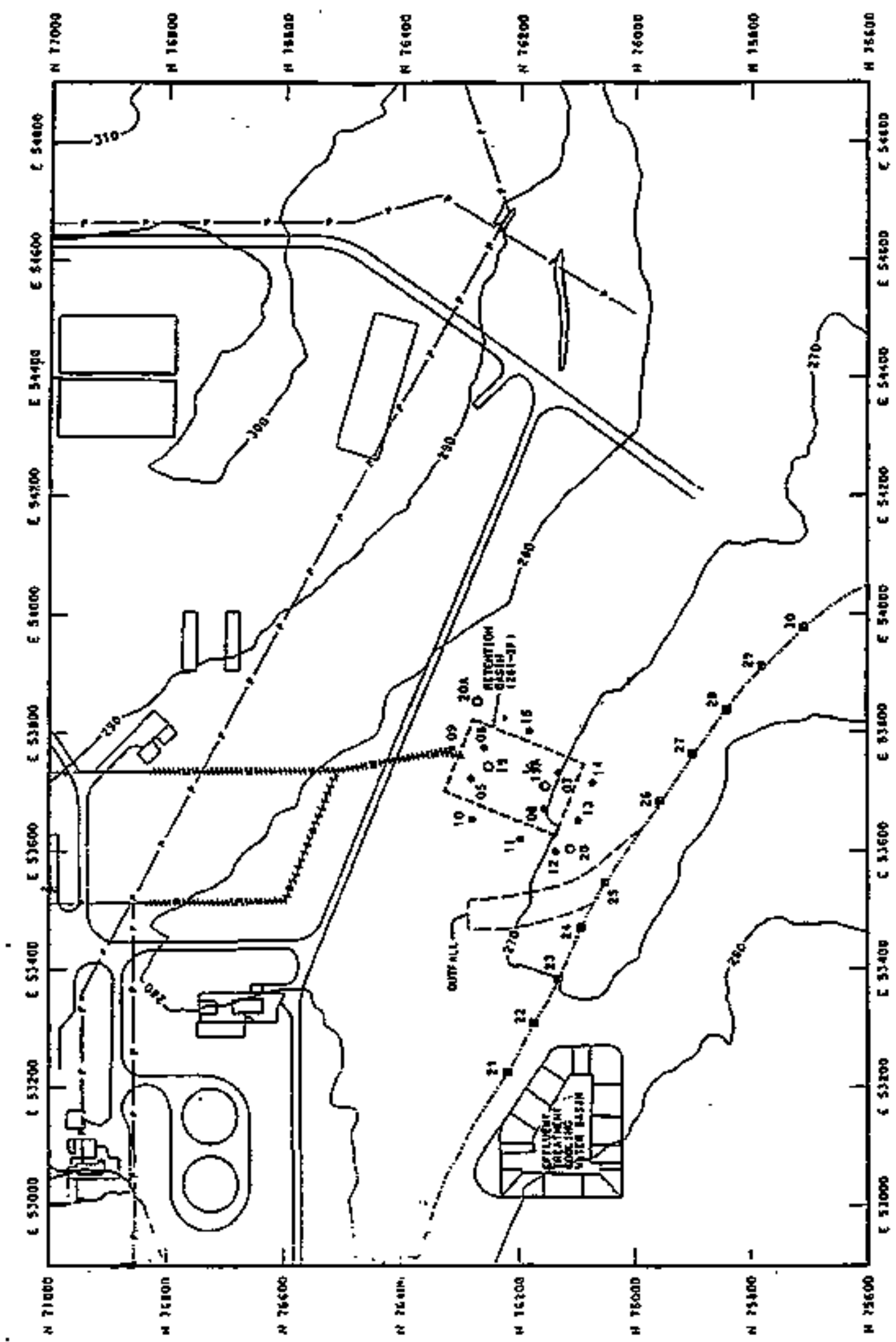

Figure I. Soil, Surface Water/Sediment, and Geotechnical Sampling Lacations 


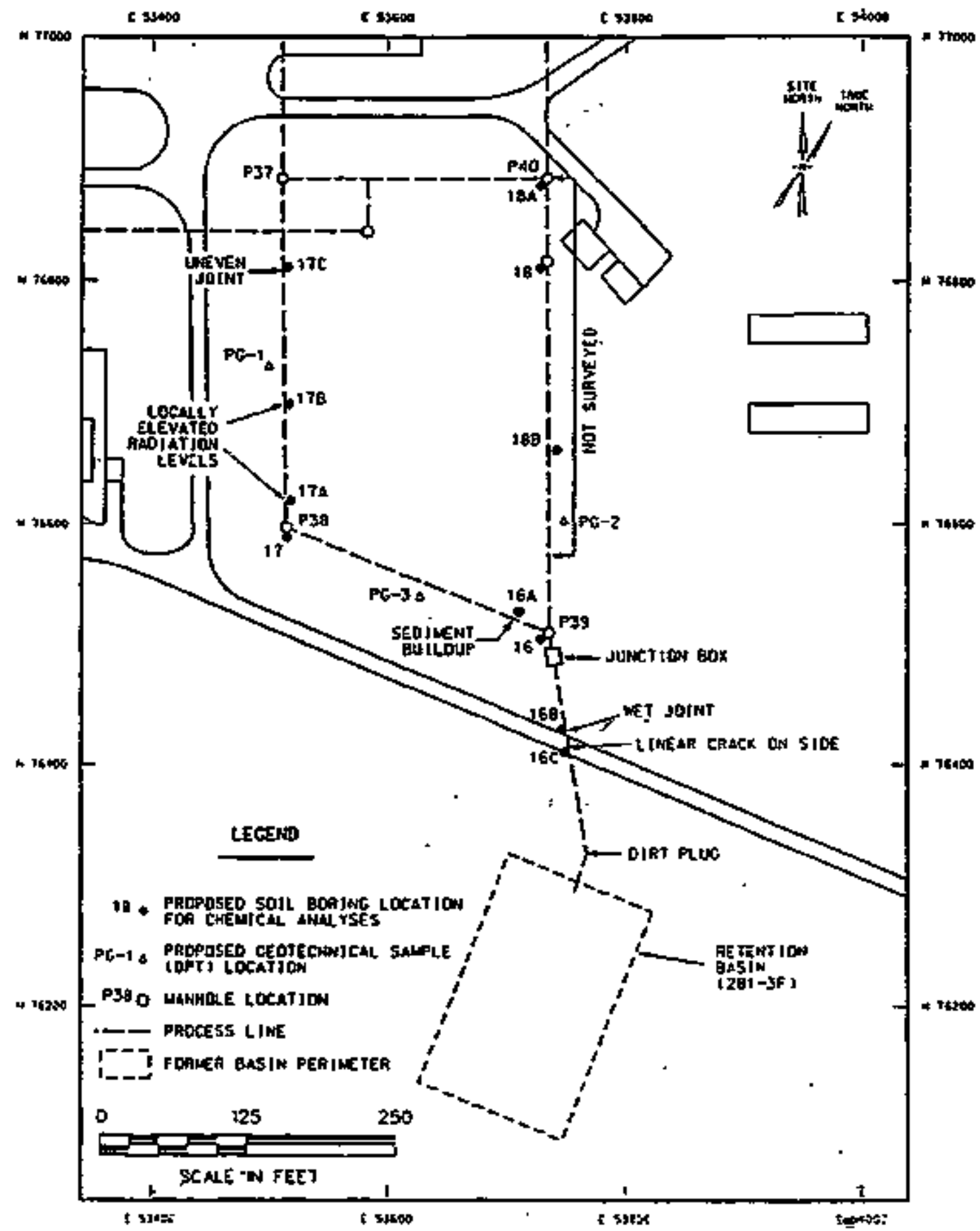

Figure 2. Soil and Geotechnical Sampling Lacations Along the Process Line 


\subsection{General}

Westinghouse Savannah River Company (WSRC) is the prime Managetuest and Optrations Contractor for the Savannah kiver Site (SRS) with ultimate authority and primary responsibility for unit/site operations that include unitsitite healh and safety. The SRS RI Project Manager (PM) is responsible for overall project coordination and reperts to the RCRAVERCLA Program Coordinztor. The Healuh and Safety Officer (FSO) (whose dhties are described below) may be the same person as the Technical Oversight (FO). The TO is responsible for monitoring dilling and/or sampiliag activities to ensure that these activities match the requirements of the RI Work Plan.

In the event thas conditions warrant that work be performed in a Radiviogieslity Buffer Area, the work will be performed under the control of the SRS Radiological Control Operations (RCO) Departuent. SRS RCO will be . responsible for directing and monitoring alt init/site health and sofexy concems assotiated with radiological materials, and when present, will be the field SRS Heakth and Sufety representative. The SRS Industriad Hygiene (II) Department is responsible for reviewing this SSHASP for compliance to IH procectures and tray perform monitoriag for non-radiological hazards in the field

The SRS RI Project Manager [WSRC Environmental Restoratton Department (ERD)] has developed tis Siste Specific Health and Safety Plan (SSHASP) in cosperation with the SRS If, RCO, Occupational Safety Programs and Assessments, Emergency Services Department, and Quality Assarance (QA).

The SRS RJ PM and the sobcontractor Froject Managers (PM) assure that all project personnel are redically fil for duty, are current in HAZWOPER or equivalent training, and have a current respirator fit test (if required). Each subconosetor PM will also ensure ther all unit/site-project persomel understand and aceept the SSHASP prior to performing work on the project. The ethain of commend for health and safety istues for this project will be SRS PM to stbeconizact PM to subeontractor TOFHSO to subcontractor feld management (FM) to the drillers. However, all personnel at the job site can stop work at any time for any bealth or saifery codectin.

\subsection{Health and Safety Oriker (HSO)}

The HSO will motitor sampling activities for the SRS RI PM who will ensure conplience with all health and saiety requirements by project personnel. The HSO wil be presept daring all drilling, excavation, and sample collection activities at the wasto unit. The SRS RI PM and the subeontractor HSO will not aliow work to begin wat the SSHASE, Radiation Work Persit (RWP) (where appikeable), Work Clearance Permit (WCP), and Excavation Permit (EP) (where applicable) have been provided to, reviewed, and signted by all appropriate field personnel. The SSHASP, RWP (where applicable), and WCP must be visibly posted at the -unitsite Before entering the controlted area of the work site, all personnel must attend a wittsite-specific briefing session, to be conduced by the HSO, on the potedtial unit/site hazards and specific requirements of this SSEASP. In addition to the tmid/sitespecific briefing, all univsite personmel and authorized visitors must sign a Datly Sigm-In/Out Log (ses Appendix C) and will be requined to provide curred docunentation of medical clearance and HAZWOPER or equivalenk training. If any documentation is not availabie or is out-of-date, the HSO will advise the personnel that requirentents stated in 29 CFR 1910.120 apply and will not allow entrance into the controlled areas of the work site. The SRS RI PM will be informed if any subcontractor personiel or thind party visitor attiempts to access the work zoale without proper documentation.

If there is any question whecher an umplanired occumence eo-she umitsits may compromise wooks health and safety, the HSO bas the authorily-to interrupt operations and to remave all personnel from the area If pactical, the SRS RF PM should be consulted before any operation is intermiplea. If wonk is stopped due to anz healih and safety concem, inmediale attemtion stould be given by health and-safery personnel woiking in cooperation with the SRS RI PM to identify and cotreet the cause of concern as quickly as possible. Any such totident should be folly documented by tine HSO and reported to the SRS RI PM. In the event of a woxk stoppage, the SRS RI PM must be kept appraised of progress in resolving the incldent until normal operations are resumed. In absente of the 
TO/HSO, the FM of each subcontractor becomes the active HSO and has overall responsibility for monitoring and ensuriting that their projest personnel will comply with this SSHASP.

Spexific duties of the HSO include, but are not limited to:

- Conduct a health and safety meeting (pxior to the finitiation of any fielowwork) in which both gerserad health and safety concerns apd those health and safety concents specifit to the RI characterization af the Waste Unit are addressed to the satisfaction of all in atrendiance. The HSO must document the workers in atendanoe by obtaining their signanures/ initals on the "Site Safety Meeting Attendance" shet in Appendix C, and any new workers must receive a documented briefing on the health mend safty coatenis covered in the original meetiog. *

- Coltabarate with SRS RCO in a fachion that ensures compliance with appropriate procedtres."

- Identify and prevent all reeogatized rokk prectices or coaditions that moy result in injury or exposure to toxic substances."

- Obtain and interpret instrument readings to determine the offree of bazard present (with the exception of majiation monitoring for bestth protection considerations, which will be conciveted by $\mathrm{RCO}$ ).

- Monitor the use of persomel protective expipment (PPE) for SRS as detemined by personal protection levels stoted in this SSHASP (except for personal protertion levels related to radiation).

- Monftor decontamination procedtures.

- Conduet pre-entry and pariodic safety training as necessary.

- Assist in the coordination of emergency response attivities for onsite personnel and with emergency support groups at SRS; ensure work site is equipped with eommunieations tapability for emergency notfifetions not obly to the SRS Operations Center but from the SRS Operations Center."

- Monitor personnel safecy perfomance for SRS to ensure that requived work practices are in ecompliance with this SSHASP during RI Operations."

- Assist with coorotoation of energency response activities.

- Monitor for SRS who will ensire compliance with, and deternine effectiveness of this SSHASP.*

- Duties to be perfonned by active subeontractor HSO if TOHSO is rot onsite.

In addition, an altemate HSO shall be iduntified if tbe HSO is umable to perform his duties. The altemate HSO will hold the same responstitilities and training requirements as the HSO.

\subsection{Project MEnager (PM)}

The Projer Manage (PlO is the individual from WSRC thonsible for overall project coordmition. The PM must make appropriate notifications in energeneies. The PM must not allow work to begin until this SSHASP has been provided to alt fietl personnel The PM must also ensure that all personnel attend a site specific briefing session condueted by the HisO. 
All personoth have the responsibility and authority to order a work stoppage should unsafe conditions exist.

In addition to the requirements set forth in this SSHASP, all work performed by gubcontractors will be performed in accondapce with WSRC 4Q (Industial Hygiene Procectures Memmal), WSRC 8Q (Employet Safety Mamual), wSRC 5Q (Radiological Control), WSRC 20Q (Health and Safexy Maruol for Hazandons Waste Operations), and the WSRC RFI Progrim Plan (latest revision), Any modifications to the SSHASP will bo docunented on the Fealth and Safay Field Modification Form (see Appendis C) and approved by the SRS RI PM and HSO prior to commeacement of uctivities.

The project manager is elso Jesponsible for coordination of site survellances. Surveillaness may be conducted by ER QA or the Suicontractor Safety Group. The ER QA Departmeat will determine that all procedures, including this SSHASP, are properfy foltowed and implemented. The Subcontrastor Safety Group will ensure that the subcontractor HSO is performing bis dutie in accordance with this SSHASP and the Enployes Safety Manmal, WSRC 8Q.

\subsection{HAZARD AND RISK ANALYSIS}

It is the responsibility of all personnel to understand and be aware of all hazardous materials that may be present during this phase of the invascigation at the F-Ares Retention Basin. The materials that have been identified to exist at the F-Area Retention Basin are identified in sections 4.1.1 through 4.1.3. During this phase of the investigation, radioactive waterials are expected to be encoubtered.

In the evept that any bazardous materials are encoantered, the principles of "As Low As Reasonably Achievabie" (ALARA) shall be followed ALARA bas three main tenants; time, distarce, and shielding. That is, it is the responsibtlity of each person to minimize the amount of time to exposite to, as well as maximiza distance from, any source of hazardous material. In aditition, if the hazatofous material is anticipated to be significant, RCO andor if may require shielding or other engineering barriens to tedoce exposure.

\subsection{Harardans Constituents}

Hazardous constinuents around the F-Area Retention Basin are antlejpated to be identical to those Identified during

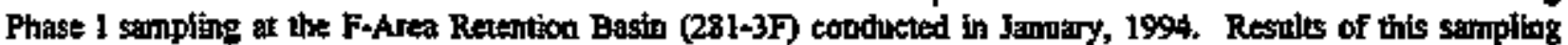
indicated that the conly contamination received by the basin were racioartive isotopes, manly C-137 and ST-90. Nether soil pas streening (July 1993) nor Phase I sampling datected any metal $\alpha$ organic conbanination at levets of concerr ar the 201-3F Retertion Basin or process sewer line, Further details of the contamination expected at the FARB are present in the following sections.

\subsubsection{Radinactive Contamination}

Radiologital contamination is the primary concen of work attivities at the FARB. Records indicated that cooling water contaminated witt radioisotopes, printarily Cs-137 and Sr-90, were sent to the F-Area Retention Basin from the Tank Farm and Canyon building on several otcasions during the operatiug gpan from 1955 to 1973 . Generally the F-Area Retention Basin revived cooling water which was too bighty combaninated to be sent to the seepage basins.

Phase I Sempling at the retention bastiv confirmed the expectations of the agare of combanination at the F-Area Retention Bastin. Ractioastive Cs-137 and ST-90 were detected ia significant quantities in the sot (60 - 100 pCi/g) as

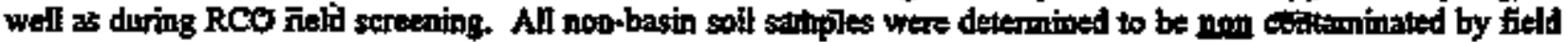
screen and laboratory abalysis. It is antiejpated thet, givet the doep water table at the bain, the radioactive coptemisants have not yet reathed the fround water. Sinceisamples will be collected from tisifie the FARB, thes sampliag will be conductod ilside an RBA. 


\subsubsection{Volatile Organic Compouads (VOCs)}

One soil gas stavey has been conducted at the F-Area Retention Besin (281-3F) wratte unit The soil gas stivey was copducted in July 1993 at the perimeter of the basin as indicated by radiological and "orange ball" matkets. No

. 'orpanic contamination was detected during this vnit screcting.

Phase I sampling at the F-Are Retention Basin (28I-3F) was eondueted in Jannary 1994. During the tinvestigation four soil samples were taken from the basin area and aralyzed for organic contaminants ineluding, volatiles, strivolatiles, and teatatively identified compounds. No orgonic componnds were detected above regulatory ation levels. However, some semi-volatile contaminants were discovered at ons location at the basin where buming was apparently condicted during remediation prior to placement of baskfill material over the basin. Since tese contaminants were not introduced as part of the Dormal operating procedare of the tetention basin they are not expected to exist inside the FARB. The list of semi-volatiles contaminants detected at the basin is given below:

1,4 Dichlorobenzeat
Eenzo(a)pyrene
Bis(2-ethylhexyl) Pythalate
Dibenizofuran
Fluorene
Fyjen:

2-Methylnaplithalene
Benzo(b) flouramtheme
Butyl Benzyl phethalate
Dietbyl Phthajate
Naphthalene
Acetone

Anthracene Bemroxh, h,ijperylene Chryseme Floumanthene Phenamthrente

\subsubsection{Metals}

Phase I sampling at the F-Area Retention Basin (281-3F) indicated the presence of small arnounts of Bayllium. This metal is not believed to be part of the process at the chinyon and therefor is believed not to have beetr introduced into the FARB. No other metal cortambants were deteeted at the F-Area Retention Basin in significant quantiries.

Health risks assoctated with motals is not expected to be a major concent.

\subsection{Exposure Routes}

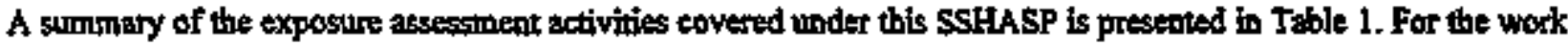
to be accomplished during the proposed activities, the maip routes of exposure is the possibility of direct contact of inhalation of coptaminated soils.

Table 1.

Old F-Area Seepage Basin Potential Exposore Pathways

\begin{tabular}{|c|c|c|c|}
\hline MEDK & HAYARB & PATEWAY & POTENTLAX \\
\hline Ground Water & Coptamination & $\begin{array}{l}\text { Direct Contzet } \\
\text { finmma ligestion }\end{array}$ & $\begin{array}{l}\text { Very Low } \\
\text { Very Low }\end{array}$ \\
\hline Surface-Water & Contamination & $\begin{array}{l}\text { Direct Contact } \\
\text { Hhunan ingestion } \\
\text { Oftsits Migsation }\end{array}$ & $\begin{array}{l}\text { Very Low } \\
\text { Very Low } \\
\text { Very Low }\end{array}$ \\
\hline Soil & Contrimination & $\begin{array}{l}\text { Direct Coptat } \\
\text { Vaporidust Inhalation } \\
\text { Transpert to Suftace Water }\end{array}$ & $\begin{array}{l}\text { Medium } \\
\text { Medium } \\
\text { Log: }\end{array}$ \\
\hline Âे & Coutarination & linhalation of ainbotne pationates & Medium \\
\hline
\end{tabular}


Harord dese to contact wilh soils:

Potential for persennel contamination due to dirent connat with soil is low since no drilliag will accerr within the waste unit (orange ball matkers). The greatest risk of contamination will be contact with drill cuttings from within and along the basin perimeter. RCO will evahate all dinll locations for radiation safety and provide coutinuous coverage at locations which may enepunter radiogetive soil contagnination.

Harards die to comatr with grownd water:

Potential for persomel contamination due to direct contact whth ground water is very low since past sanpling and current aransport models indicated that no contamination has reached the gromend water. The greatest risk of contamination will e contex with ground water from down gradient wells. RCO will evaluate all dill locations fro radiation safety and provide spot coverage to ctreck for groumd water contanination.

Hozords due to Organie Vapars:

Potential for persormel exposure to organtc vapors is very low since no organic chemicals are known to have been released to the FARB. The presence of organic vapors will be mosttored with as on-sit OVA or HNit by the TOIHSO at the discretion of IH and any decision to wear respitators must be ruade by IH in cooperation witb the TOHSO.

\section{$4.3 \quad$ Environmental Heqult Considerations}

\subsection{Cold Stress}

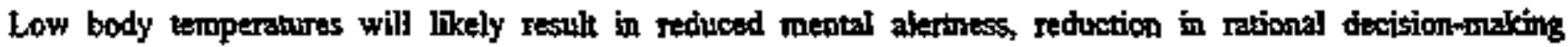
copalitity, loss of consciousness, of deatit.

Mild to severe pain in the extrenities may be the first early waging of danger to cold stress. Daring exposure to cold, maxinum severe thivering develops when the body core temperahure has fallen to $35^{\circ} \mathrm{C}\left(95^{\circ} \mathrm{F}\right)$. Useful physical and mental work is lingited when severe shivering cecurs. Becanse prolonged cold exposure at tepnperatures well above freezing can lead to bypothermia, whole body protection must be provided. If work activities are performed in temperatores betow $40^{\circ} \mathrm{F}$, adequate insulating elothing to maintain core temperaturt must be wom by all workes. Darmp or wet ellothing may contribute to cold stros. All workers sbould be aware of the effectof witd chill on exposed skin. Appendix B contains adiditional cold stress information.

\subsubsection{Hat Stress}

The major disorders dve to heat stress are beat eramps, beat exhaustion, and heat stroke. Hear cramps are painful spasms which oceur in the muscles of wotkers who sweat profustly in the heat and fink large quantities of waket, but fail to replace the body's lost salts and electrolynes.

Heat exhaustion is characterized by extreme weakness or fatigus, dizainess, nausea, and headache. In serious cases, a worker may vomit or lose conscionsness. The skin is elammy and moist, complexion pale or fleshed, and the body temperature can be normal or slightiy higher than normal. Treatment consists of rest in a cool place and replacement of body water and salt lost by perspiration. Mild cases may retover spontaneousfy with this treatment. Severe cases may fícpirie care for several days. There are lo permaneat effects.

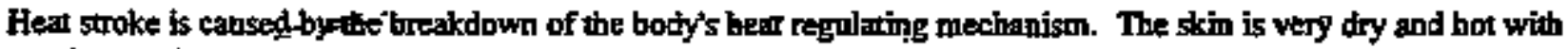
a ret $\alpha$ blutsh appearance. Uncomsciouspes, mental confusion, or convulkions uray acent. Without quick and adequate treaturent, the resolt can be death or permanent brain damage. Medical assistance shouid be given quickly. 
The person shoutd be moved to a cool place. Body heat should be redaced antifiktally by soaking the person with water and vigorous fanming.

Wearing of protective clothing, suth as Tyvet, KlenGuard, polyetbylene coated Tyvek, or saran coated Tyvek suits may increase the danger of heat stress.

Workers should be aware of the symptems, dangers, and appropriate prevealive ind treatment measures associatred with heat stress. Appendix B contains additional heat stress informatibsi.

\subsection{SAFETY AND HEALTH TRAINING}

\subsection{Geocral}

Training of each workes ts to be werified by the HSO prior to site entry. In accordance with the general site policy and the guidelines set forth in WSRC 20Q, "Health and Safety Manjal for Hazarious Waste Operations," Procedare E, the following ts required;

- GET, if enployee is on site for more than 10 days or needs to have unescorted access to the SRS.

- Radiation Worket II Training as required by WSRC 5Q.

- RCRA training if the stite is a RCRA Treatment, Storage or Disposal facility (TSD) or there is a potential for generation of harardous wate.

- HAZWOPER or equivaleut troining, outlined below, including Op-the-lob Training (OST.)

Table 2. Training Requirements for Waste Sits Workers

\begin{tabular}{|c|c|c|c|c|}
\hline Individual & $\begin{array}{l}\text { 40 Hoor } \\
\text { HAZWOPER }\end{array}$ & $\begin{array}{l}24 \text { Hour } \\
\text { HAZWOPER }\end{array}$ & $\begin{array}{l}8 \text { Hlour } \\
\text { Supervisor }\end{array}$ & ONT \\
\hline HSO / Altemate & Required & & Aequimed & 3 Day Required" \\
\hline $\begin{array}{l}\text { Techrical Field } \\
\text { Oversight }\end{array}$ & Required & & & 3 Day Requited \\
\hline Laborer & Retulired & & & Required" \\
\hline $\begin{array}{l}\text { Onsite } \\
\text { Management and } \\
\text { Supervisors }\end{array}$ & $\begin{array}{l}\text { Requined, see } \\
\text { exceptions } \\
1910.120 \text { (e)(4) }\end{array}$ & & Regaired & 3 Day Required \\
\hline $\begin{array}{l}\text { Low Rusk } \\
\text { Workers"* }\end{array}$ & & Required & & J Day Required \\
\hline
\end{tabular}

- Laborers without OIT must be supervised by the HSO for at least three days when they begin a HAZWOPER.

** See 29 CFR 1910.120 (t) (3) (ii-jii)

HSO trating also requires First AidCPR and Bloodborne Pathogen.

\subsection{Testispecifit}

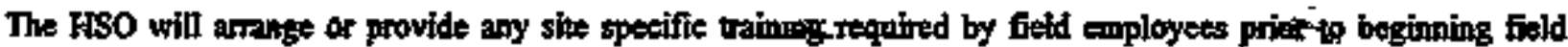
setivities. This will constst of a review of the specific bapards of concers, risks, symptoms af exposure and an overview of the SSHASP to include delineation of woikzones, acess, decontanination protreols, sefety and emergency procedures and emergency contacts litted in section 8.5. The StS custodian, RCO, H and ERD projest manager äre javited to this training, if conducted by a subeontroteror. 


\section{Trabing Docnwentation}

Docturentation of all applieable health and safty trainfing will be maimsained and will be prowided to the SRS RI PM witin a reasonable time upon reguest. The project STR and/or Waste Ste eustadian will maintain records.

\section{$5.4 \quad$ Vsitors}

Vistitors are responsibte fer protiding training recorts to the ERD project manages gad/or the HSO before entering the worksite. The HSO will review unit specific procedures and hazaids with the visitors before acesss to the site is allowet.

\subsection{PERSONAL PROTECTTVE EQUTPMENT (PPE) AND PROCEDURES}

\section{I General}

PPE will be determined by WSRC RCO and TO HSO taling into consideration the level of contaminalion expected and detected (reference WSRC 20Q "Health and Safety Menual for Harardous Waste Operations," Procedure G). Non-inirusive operations do not require any PPE other than appropriane safety gear as determined by standard SRS procedures. Workers within the exclusion zone will wear PPE as direeted by WSRC RCO and/or the TO HSO and workers outside the exclusion zone and vistiors witl begin in Level D PPE. The following guidelines shall apply to all trisks:

- PPE shall be available at storage locations which may vary for difierent projects and will be diescritied by the HSO at pre-entry briefings. The HSO will describe appropriate doming/doffing procediures.

- PPE stall be inspected by the wearar prior to ase. The project manager or designee shall arsume that respinators are supplied, ingpected, and maiptained by the WSRC respirator faetility.

- Decontranination procedores are described in Cbapter 10.

- PPE that cannot be decontaminated shall be stored in a satellite or staging area within the wast: unit until pickup tor disposel. ERD is responsible for this funsion.

ERD will evaluate this PPE program lssing infondtion collected from interviews with wotkers,

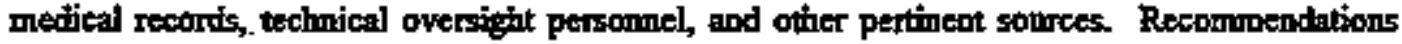
and changes will be incorporated as needed. The SRS RI PM is responsible for preparing periodic reports describing results of evaluations and maintaining reports as part of the project records.

\subsubsection{Respirator Certitication}

All personed with the potertial of exposure to hammfol airborne contamination must have medical respirator . certification by a qualified physician, and respirator fit prior to respirator usage.

\subsection{Self Contained Brenthing Apparatus (SCBA) Certicieation/Tritium Suit Certilication}

Before an individnal may enter an area desigmated for use of SCBA, the individual must bave documetted medical qualification for SCBA utilization; training in the proper inspection, maintenance, and use of the SCBA unik; and must receive a unit-specific salety briefing. The unit-specific betiefieg addresses known levels and types of bazards at the unit, the "boddy systern", operations to be performed, appropriate emergency procedures, and the potential consequences of failing to maintain proper respiratory protection. Falilure to meet efry of these requirements

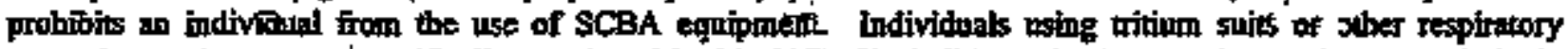
protection equipment nqi specifically mentioned in this SSEASP shall be trained to use that estaipment and chtatn concuntence from ERD beforte using that equipment, excepsin curdigencies.

\section{2 .3 Respirator Selection}

Respirator for non madoamiye spories 
Respliatory protection or other controls may be required in the event that ambient concentrations of organius are detected ar the wotk site. In the tvent that ambient or changing concedutions of organics are detected wotk will be stopped until the source of the readings is identified. If readings persis, the HSO in consultation with II will determine if respiraishs are required. Respirators are isqued per 4Q1.6, Procedure 501.

Respirators for atithome redioactive spectios

In the evant that aiboune fadioactivity is deteeted, the SRS RCO will stop the job and reevahuate the appopriats protection prior to resumpition of field activities. Respiratory protection reqquirements are detaled in WSRC SQ Chapter 5, Fart 3.

\subsection{Non-Respiratory PPE}

\subsubsection{Non-Introsive Operations}

For non-ininesive activities, no exposure to contaminants is astictpatted. PPE and safty equitinent should be sefected according to WSRC 3Q5 (Hydrogeologic Data Collection) and the WSTRC RFIRJ Program Ptan (batest Iovision) and other staindard procedures appropriate to the task.

\section{3 .2 Intrasive Operations}

For intrusive operations, Level D clothing is appropetate unless diusted othesvise by WSRC RCO. Hard hats and safety glasses with side stields sbould be wed during th ditling activities. If determined nscessary after monitoring by the HSO, Level D (modifed) clothing (Tyvek, boots and gloves) and equipwent must be maintained throughour the dination of the work in the exchosion zone. The following minimum PPS (or equivalent) anc available to all persomeit:

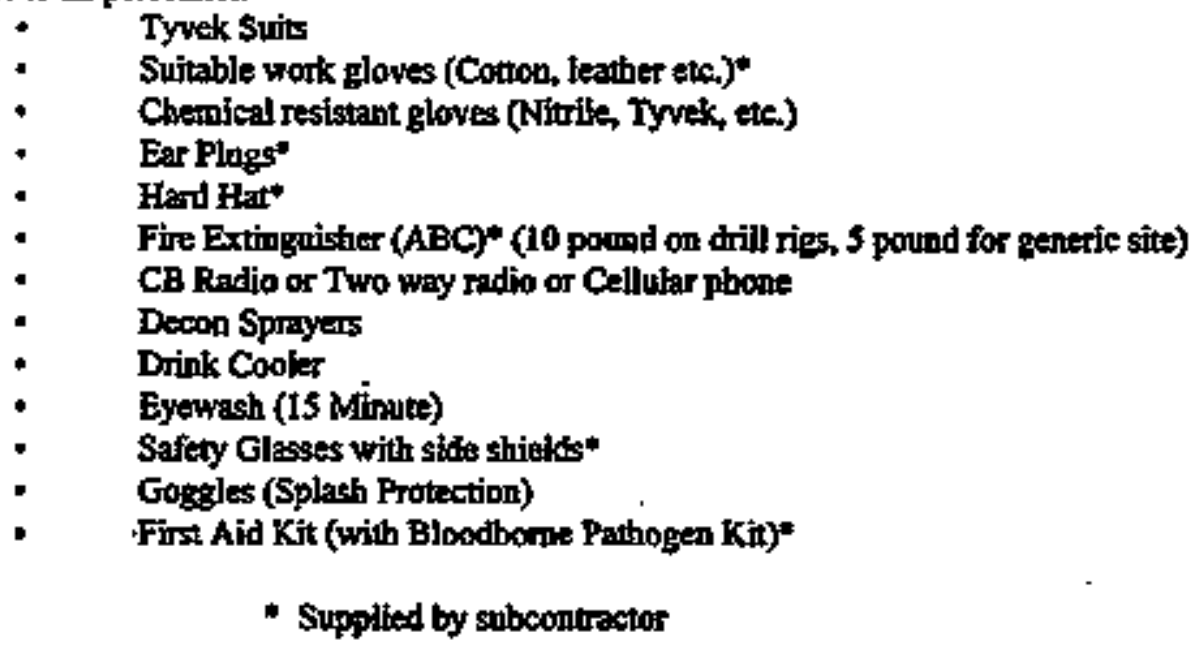

\subsection{MEDICAL SURVEILLANCE}

Employes who may be exposed at or above the permissible exposure limits (PEL) or wear a respirator for 30 days or more per year must be covered by a medical surveillance program as required by 29 CFR 1910.120 and deseribed in Procedure F of the WSRC 20Q. "Health and Softy Manual for Haradous Warte Opierations". All field personnel mest present evidence of finess for duty, siponed by a phytivian

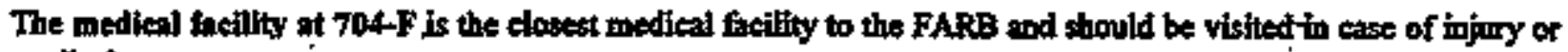
medieal emetgescy: 
Monttoring of temperatbere for heat/cold stress will be performed is necessary by the HSO by calling the nearest weather station for temperature and relative humidity measurcesents. Should weather temperatures and hunidity warrant, HSO shall trike all necessary meagures to minimize risk of beat stress and heat stoke (e.go, beavy work shall be periormed in early and late hours of the day, HSO shall detemine if adequate filids and shade are 3vailäble for workers, work will be doge in work-sest cycies, etc.). The Feat Stress tedephons number is 7-0576. Guidelines for Heat Stress and Cold Brpostere are desuribed in Appendlix B of this SSEASP. Eact monitoring instrument will be ealihrated as required by IH and RCO as desertbed below. Calibrations will be reconded in a loghook.

\subsection{Amblent Afr Moniltoring}

Air monitoring will be wed to telentify and quantily airhorme levets of hapardons substsaces in order to determine the appropriste level of employee protection needed on site Volatile organie compounds (VOCs) are not known nor suspected to be present in the FARB. Initial sirborne monitoring for radipactlvity at esch borebole sitte will be provided by RCO. To confire levets of airberre chemical contaminants, any Immediete Dangerous to Life and Feolth (IDLH) abd otber dangorous situations, such os the presence of flammable atmospheres, oxyten-deficient environmemts and toxic kevels of airborne contaminants monitoring at each borehole site will be provided by the smbeontrector and will be conducted prior to drilling each borebale. Monitoring finstroments for the inition sarvey will include a combutible gas indieator, orygen detector and Organic Vapor Analyzar (OVA) or HNu, and whtl be used in the orter presented. Monitoring for VOCs in the persomel breathing 20ne will be conducted every time a sample is taken with an OVA or HRin in every boring to the water table and periodienlly to the totel depth. If conctintrations of Vocs above background are detected, work will stop and personel will leave the erejnsion zone iramedistely. Work wili not contibute until the HSO has assessed the situation to accommoilate the elevated VoC level.

If radkanuclides are detected in samplies collected inom the bareboles, to monitor potentiof airborne radiosctivity, an air sampling network will be extablished to form a perimeter around eacb borkhole location. Sample collection will be provided by a portable pump attoched to a power source, by which the sample is sontinuously collected onto filter paper. The collector will be pyobed by HP during drilling with HP110 and Ludlum monitoring instraments at a frequency determined prior to investigation ativities. In the event that onacceptable emissions are detected at the work site, all work will stop and personnel will keve the erclusion zone tmmediately. Work will not continue untli HP personnel determioe the extent of eontamination and the personal protection required to safely complete the task.

\section{Personat Monitoring}

For the proposed FARB ipvestigatiot activities, the postentiat radiologital dose to workers is estimated to be mininal. Exposure duriog field antivities is expeeted to be a navimbm 10 mrem per person in a two week period. In addition, personnel will be notilied if any nearby facility acenrrences warrant the temporary suspension of work to ensure that personnel are not exposeti to unnecessary contamination and/or rodintion.

SRS RCO will provide coverage for work by surveying for sadiatiou sad radioactive conterination. Coverage will be provided contiauoosdy for drilling gnd sarmple collextion activities in the FARB perimeter fence and intermittently for the remainder of buvestigation actlvities after an filtial work site survey. Contamination and radiation wil be detected by portoble instrumenti at each FARB work site. For clearance purposes, soil eore and water samples will be taken to a kCO facility lab for amalysts for radiosetive material. RCD coverage will not be required below the following levek:

- No degectahle radtotton shove naturalfy fecurting background. (Natural background not to

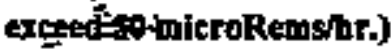

- Smearable cantamination less than $20 \mathrm{dpm}$ ajpha and less than $\mathbf{2 0 0} \mathrm{dpm}$ beta-gamma and 10,080 dpm tritium. 
Monitoring for radionctive contanination of soll and equipment used and exposed doring drilling will be performed by RCO axing portable instrumeots at the work site. When drilling and samplling of a borehole are eompleted, RCO wil (if no contamination is detected during these processes) declore the work site "clean" (free of radiation and contamination). If contamination is detected dering fald activitios, protective clothing and/or dosimetry reguirements will be upgraded to protect personpel from imereasted levels of contaminatios abdior radiation, aceording to procedures outitined in WSRC $5 Q$ L.2-217V (TLD Badges Types and Usage), WSRC 5Q 1.2-218 (Dasimeters) and WSRC 5Q. Conteinment of the pork site will ako be required for work to continut, such as a SAS wiadbreale, wind speed limftations wnd weather condition limitations.

Whole body counts and biologkes sampling may be conducted during certain work activities at work sites identilied by SRS RCO. The need for and frequency of this monitoriag will be established by SRS RCO, based upon the work plan. SRS RCO with provide guidance on persopnel monitoriag and bioassay requirements and IH for potential non-radionctive chemicsl exposiares.

If a change facility is aot in close proximity to a driling work sits one will be estabilised and a count rate meter instolled if electrictiy is provlded to the change facillty; otherwiss, RCO will perform the necestiry personnel monitoring.

\subsection{Non-Radfoactive Specles (this section applies to irtrustive operations only)}

An OVA or HDNu will be used to detect levels of organic vapor above background. Organic vapor monitoring will be performed by IH or the HSO at the start of each wotk day andior new location. HNa's are calibrated in actordance with 4 Q1.2 Procedure 119.

Working requirements when organic vaposs have been detected will be determined by the HSO and HH. If explosive conditions are detected, work will be delayed until appropriate engineering measures can be taken.

\subsection{Radioective Species (this section applies to intrusive operations only)}

SRS RCO will detemine the proper monitoring equipment to be used to detect atpha, beth, and garama tmitters. At a minimurn, WSRC RCO will conduet a pre-job screening to identify types and levels of contanination at the FARA. The information will be utilized in developing the RWP for the bastin activites. The job specific RWP, if required, will address the types and frequetecy of tadiation detection required at the FARB investigation activities. If work is not performed withts an RBA, RCO will monitor intermittently for the presenco of contamination. Some samples may boundlected for combing with scaless. Field radiation manitors are elibrated using WSRC 5Q1.3 Procedure 144 and scakers are calibrate using WSRC 5Q1.3 Procedures 404 and 405.

\subsection{SITE CONEROL}

Thit section cpplies onty if conditions become such that the HSO, ERD Prolect Monger, RCO ardlor II persannel deem it necessany (i.e., if contanination has been detected). Fork will be conducted in actordernot with MSRC 20O. "Heath and Sofety Martwal for Wazardots Waste Operations," Procecture D.

Organization will consist of three gentral zones of operation on each work site (see Figures 4 and 5) established to reduce potential migration of work site substances and the ritk of personnel exposure to work site materials. These zopes will be nanted as follows:

- Exchasfon Zone:

- Contzantotion Redoction Zone;

- Support Zone. 
Prito to any wotk antivity, zone tonodaries will be estabilished by the HSO and RCO if necessery in accordance with Procedure 9 of the 8Q Manmal. Pessonnel entering the work zope will be required to stigh in and out on tise "Work Zont Entry and Exit Log" form in Appendix C of this SSHASP.

The intibia exclusion zone will be an area centered around the borehole witi a minimtum radins of 10 feet. Boundaries for other activities may be establisbed by the HSO and RCO. After work begins and monitoring of the work site bas been conducted, the extent of the Exelusion Zone may be revised with the approval of the HSO and RCO. Zone boundaries may requine modification based on changes in weathes, conditions in the Exelusion Zone, new dath, modifications of the RI work pkan, etc.

\subsection{Extusion Zone}

The Exclasion Zone is that area where active operations take phace. This area is considered potentially contaminated, and all personnel within the anea will unfilize the bevels of personal protection preseribed below. A checkpoint will be established at the periphery of the Exchusion Zone to regulate the flow of personal and equipment into and out of the area. The HOT LINE will be posted or well-defined by geographical and physical boumdaries. The Exclusion Zone may bo subdivided inm areas based upon environmental measurements, the results of provious surveys relative to the concentrations of known or supected substances, and expected on-site work conditions. Area delineation correspouds with the level of protection repuired for entry; for exampte, Area D of the Exelusion Zone requires D-Level persoral protection. Critoria for detemining area delingation are summarized as follows:

Area A is that ares where the highest leveis of potential contamination may exist and is designated as the area where maximum respiratory (SCBA), skin, and eye protection are required (i-t., Level A personal protection).

Arta B is that area where maximim respiratory protection (SCBA) is required; bowever, there is a low probability of demal toxicity (requires Leved B personal protection).

Area $C$ is the area where respiratery protection of a lesser degree than the criteria establisbed for Areas A or $B$ is required (full-face, negative-pressure, ain-purifying respirator with appropriate cartidges) and the probability of skin contamination by toxic materials throngh dermal rontes is unlikely (requires Level C persomal protection).

Aree $D$ is that arca where respiratory protection is not a requirentent.

The level of personal protection to be utilized in the Exchesion Zone will be Level $D$ unless otherwise direted by. the HSOTor RCO. If direted by the HSO or RCO, a persor certified in SCBA operation will be present during drilling/excavation, in case of emergency.

The Contamination Rectuction Zone is a buffer between the Exchesion and Support Zenes. It is structured to prevtrit the rijgation of substances from the Exclusion Zone to tress where these substinses are not present All eqpipment and personed cleansing procedures are performed within this area The boundary betweep the Reduction and Support Zones is known as the Control Lime and is designet to separate areas of potential contandination trom elean areas. Entry from the Support Zone fnto the Redpction Zone will be through a controlled aceess point Petsomet making this entry will be wearing at a minimum, personal protection one step below thar which is prestribed for the Exctusion Zone. Exit from the Rectuction Zoune to the Suppost Zone rexpires the removal of any suspected or known work site substances through compliance with estabiabled cleansing procedures. 


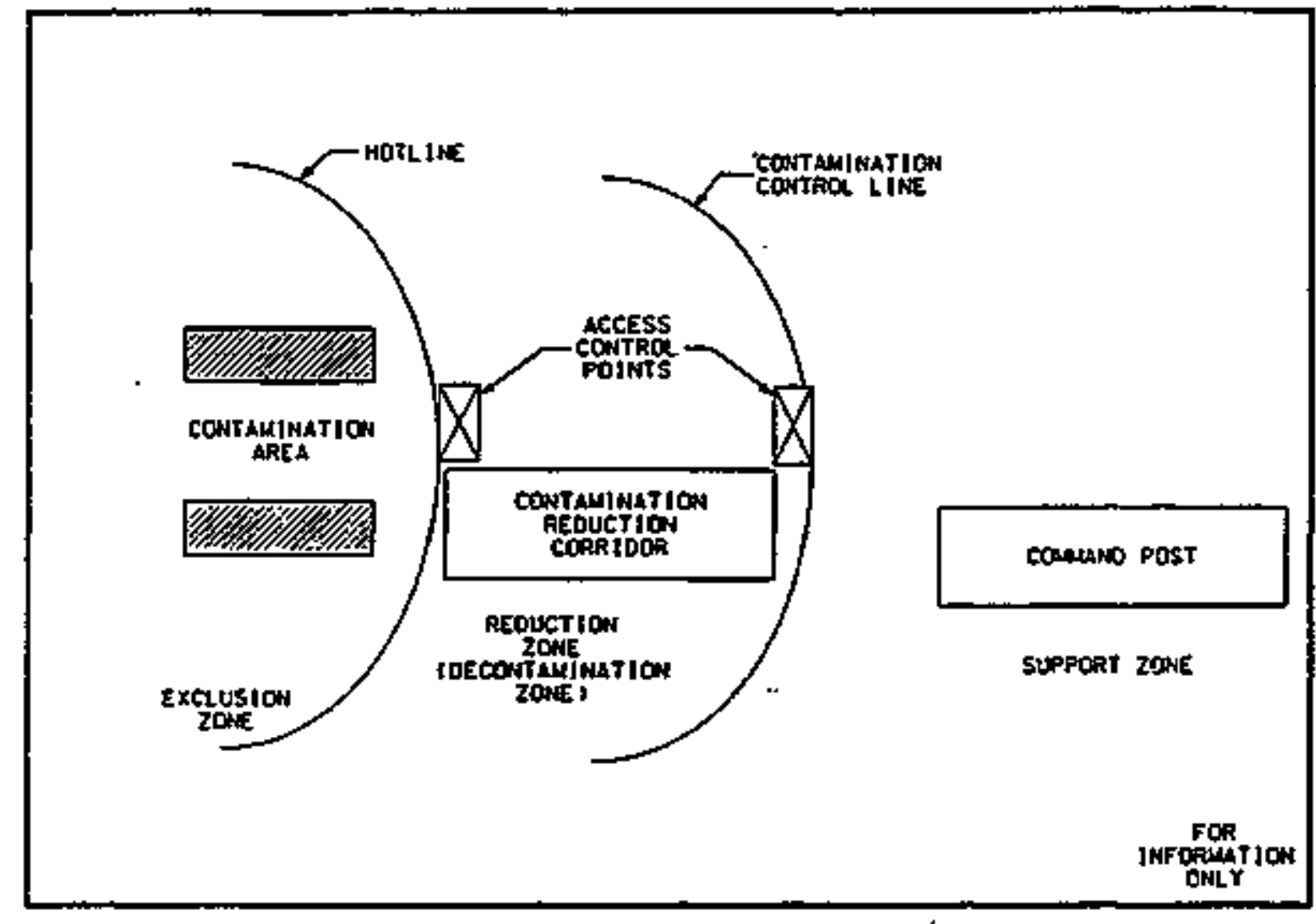

Figure 3. Sthematic of Work Zones. 


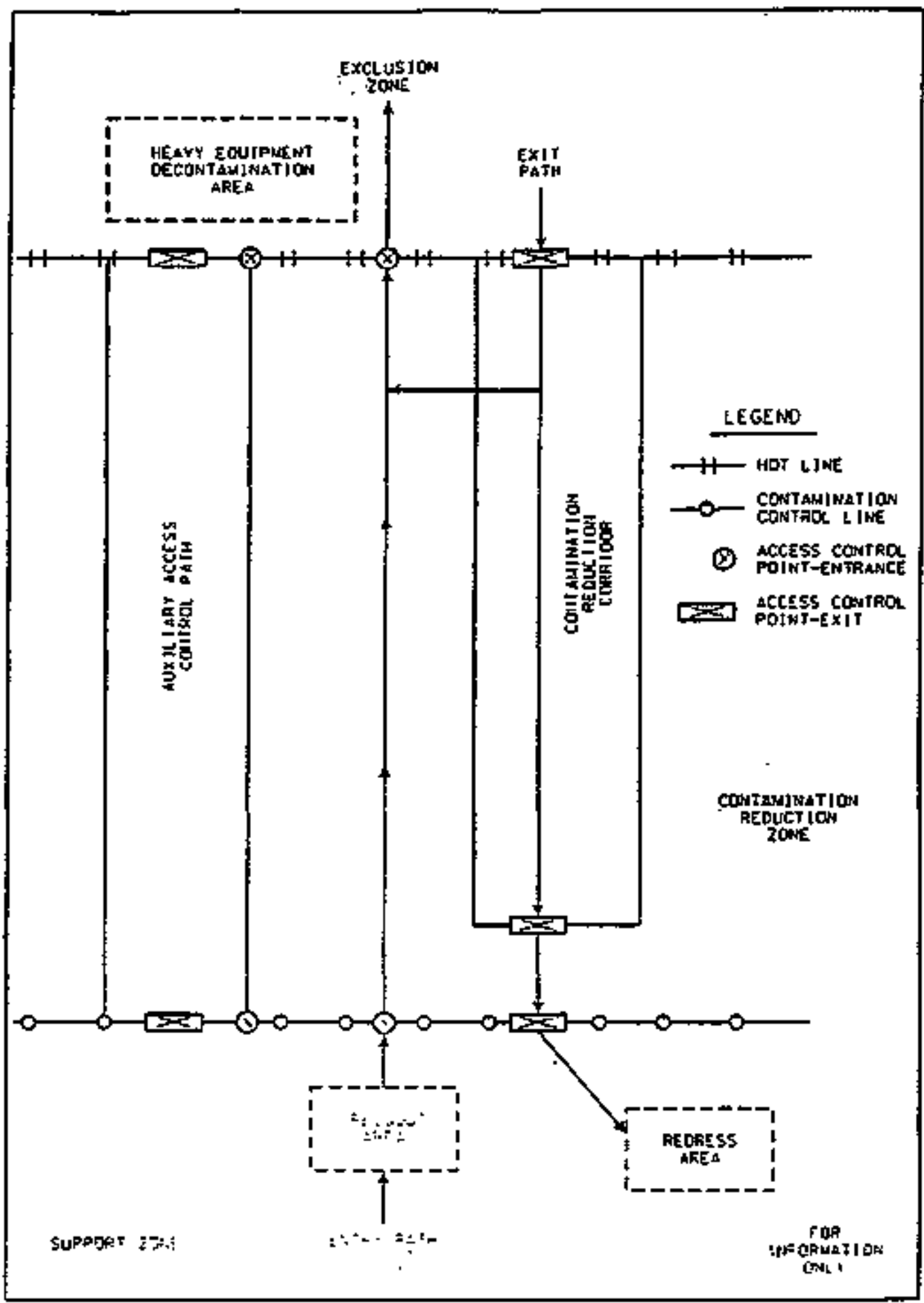

Figure \&. Schematic for Perstsnnel/Equipment:Control. 


\subsection{Suppart Zone}

The-Support Zone is considered clean, and generally is the cutermost of the designated areas. The command post for feld operations, fiss aid station, communicattons, and other elements necessary to support site opuations are contained within this area, and nofmal street elothes (Level D) are generally worn. Command post location-within the Support Zone will be baed upon a mumber of factors, inchuiing wind direction and accessibility.

\subsection{Emergency aud Support Equipment}

This section applies only to the extent directed by the HSO, ERD Project Manager, RCO andor IH persomel.

Adequate cmargency equipmest will be available to protect all woik site persometi from known of unticipated bealth and safery hazards. Before entry to any work area, all personnel and visitors will bo atvited of the necessity for and utilization of emergency equipment As a minimum, emergency ecpipment to be made avaikable on-site will inalende the following during all astivities:

- First ajd kits (16 unit as specified in National Safety Council Data Sheet No. 202 or equivalept);

- Portable eyewash stetion meeting ANSI 235801, 1986;

- Decontamination spratyers, water, and soap;

- Respirators (including a minimum of two SCBA, if applieable; see Seetion 62) (ANSI Z8821980);

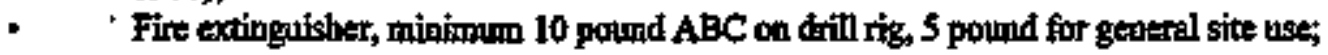

- Appropriate monitoring equipeneat (see Chapter 8.0);

- Communication equipmeat (nwo-way emergency radios or celtular belephones approved for use at SRS).

\section{SUPPORT EQUIPMENT}

- Drink cooler with water.

- PPE listed in Chapter 6.0.

\subsection{General Health and Safety Measures}

The following generad operating procedures will be foliowed by afl field personnel. These precautionary measures are desigued to reduce the risks of inadvertept of accidental radiologieal and chemical exposure of injury onring field operations. Instucting respective enployess in safe work practices and emerpency procedures is the respousibthity of the HSO.

\subsubsection{Personel Prectutions}

Ealing, drinking chewing gum or tobacos, of any practice that increases the probebility of band-to-reonth transfer and ingestion of material is probibited in any RBA, Exelusion of Contimination Rechetion Zobes. However cold water and disposable cups may be locaned in the decentranination inea such fitat employees will have access to water with only removal of gloves, hat, and respirator where used except when in an RBA. Smoking is not allowed at my tine in the waste sine.

Conbert lenses shall not be wors in Exchusion and Contamination Reduction Zones.

Fands and face muist te thoroughly washed upon leaving the-wock area; however, if in an RBF washing might not. be needed. Wheneve decontanisuation procedures for outer garments are in effect, the entipe body should be thotoughly washed as soirts possible after the protective gapent is removed.

Avoid contact with contaminated or suspected tomarninated sufaces. Whenever possible, woid walking through putides, pools, mud, eas, Avoid kneeling or sitting on the ground, equipment or drums. 
Pessonal articles shall be probibited in Exchusios and Contamination Rechetion Zones.

Medicine and aleobol can exacebate the effect fiom exposure to toxit chemieals. Aleonolic beverage intake is prohifited at SRS and should be minimized or evoided on off-wark hours during field operations. PTeseribed drugs should not be taken by personnsl during field operations where the potential for ahsorption, inialation, or ingestion of toxic substances exists umbers speetikcally approved by a qualified physician. Do not work when th.

\subsection{Drilling Soltety}

Drilling safety is the responsibjlity of each member of the urilling crew. Standard operating safety procedures shall cosuphy with the Westinghouse Savanoah RJver Company Mabual WSRC-3Q5, "Eydrageologic Data Collection," Chapter 2 and DOE Onder 54\%3.4.

\subsection{General Operating Procedures}

- Salety protocots for ord and hand signals witl be established

- All unfarmiliar operations will be rehearsed prior to implementation.

- Personnel involved in active operations will work in pairs, "buddies."

- A minimam of "buddies" plus n thirid party equipped al the same level of personal protection as the fleld team persomned (for emergeacy response), will be reguired if conditions requibe SCBA andior fully encapsulated suits. Commonieations between all work site petsonmel will be maintained at all times.

- A wind indicator will be provided to determine upwind escape routes dering intrusive operations.

- Only necessary equipment will be alliowed in contaminated antas.

- Appropriate areas for support, contasination reduction, and exclusion will be establisbed and will be described at the initial operation spectfic (i.e., drilling, excavation, etc) bealth and safery meting.

- The HSO will condnct, at a minimum, weekhy bealth and saftey meetings.

- Job sítes will be maintained in a clean, safe, and orderly mantaer. Daily cleanup activities shiall be performed, including the following:

All stored matefial that witl be used in wotk activity will be stored square to the site. An mobstructed walkway at least three feet wide will be powided for atcess to materials.

All loose trateriduwill be picked op and stored away from the work activity in a neat, ordierly manner.

All work areas, walkways, and material storage areas shall be kept free of trast and debris.

Common sense of the worker shonid prevail in preventing cold stress. This may include ingostion of warm beverages and/or removal of wet elothing-

Guidelines for control of heat stress in WSRC $4 Q$ are to be followed. Heat stress cuntrils are required for continuous work longer than 1 hour when tenperanure is above 85 degress F and humifity is above $60 \%$. Temperatare and other weathes-related information can be obtained from the Weather Cepter Anatysis Lab at 51182 or from the SRS Opernions Center (SRSOC) at 3-2117. The following steps can be taken to roduee heat stress:

\footnotetext{
- Acetingate la body.

- Driak mase liquids to repiace body wakertiest during stweating

- Increase salt consumption (salt tables are not recolmineoded).

- Wear personal cootiag devices.
} 
- When usigg supplied-air respirators or suits, equip them with a vortex tube (maximugn length 50 feet with a plastic stiti).

- Follow appropriate worktest cyele.

\subsection{Trenching and Ereavations}

Excavations is excess of 4 feet deep must meet the requirements of WSRC $8 Q$ Procedure 34 and 29 CFR 1926, Subpart $P$. The project manger is responsible for prowiding an approved Vessel or Copflned Space Entry Permit per the requirements of WSRC 80 Procedure 33.

\subsection{DECONTAMNATION}

Personvel decontamination is highly taskespecific and will be detemined by the HSO, RCO (if applicables, and the ERD project manager. It will be consistent with WSRC SQ, "Radialogical Controls Mannigl" and WSRC 20Q, "Heath and Safety Manual for Hazardious Wate Operations,", Procedure K. Decontaminatiop procedures will be perforned on toals and equipenent taken from the Exclusion Zone, if monitoting inditeates the presence of hagardous stabstances. The equiptrent will be decontaminated at the end of the project or between indivitual work operations as netedeot.

For all egress from the Exchwsion Zops, shations for decontamination of personnel will consist of the following:

- RCO clearance point specific to needs

- Equipmentit drop;

- Boot and glove disposal (if disposable items);

- Coverall drop;

- Respirator drop (wash and tinse periodically) applies to chemjealcontanination only; and

- Personrel shower, if work stre conditions warant.

If contarisation is detected or suspeted, cleansing procedurs for Level D will include the following:

- Decoptamination solutions: spep and water, and water rinse;

- Place equipinenttools on teble or groundeloth;

- Remove hard has;

- Wash outer work gloves and remove duct tape;

- Remove outer work glowes;

- Remove Tyvek sult and place in plastic bag;

- Remove Safety Glasses

- Remove imer surgieal gloves and place in plastic bag;

- Troroughly wash hands, arms, and face; rinse with clean water, and

- Pump alt decontanination fhrids into trums, apply appropriate labols, and place on pallets for removal from work site.

The HSO may modify the above decontanimation procedure commensurate with level and variety of PPE and contamination encountered. Equipmeat decontamination procedures are described in Appendix A for specific trorusive tasks. Spectite requirement for waste diposal WSRC-MM-90-138, "Waste Disposal Manual", WSRC IS, "Waste Acceptance Critein", Procesture 6.3 of WSRC 3Q, "Eiviromental Compliance Manual", and Chapters 3 and 4 of WSRC SQ, "Radialogisal Comtrols Manual".

Equipment and matedajsispected to be contaninated with firioativiny will be handled according to standard SRS procedires. 


\section{0}

\section{EMIFRGENCY RESFONSE PLANS}

As specified in WSRC 20Q, "Health and Safety Mamual for Hazardons Waste Operations," Procedure L, a plan is fequined to handle anticipaxed emergencies. An emergency situation is considered to exist if:

a any member of the field cretr is injured in an acejdent, or exhibits any adverse effects or symptoms of chemical, heat, or cold exposure,

b. safety monitoring indicates unit conditions more hazardous than antictpated,

a an immedtan danger to lift or health exists.

\subsection{General Emergeney Procedures}

in the event that any member of the field crow experiences any adverse effects or cymptoms of exposure while on the scept, the extire fietd ctew will immediately balt work and act aceosdiag to the instruttions provided by the HSO. This ineludes heat or cold exposire as well as chemical/radiological exposure.

The discovery of any condition which would sugpest the existente of a stabation more hazardous than anticipated wil result in the evaeustion of the field tean and re-valuation of the hezand and the lovel of protection required. Contact shall be mode to appropriate departments (Red Con, IH, and Subeontractor Safery Gromp) to identify the source and assess condiaions prior to restating work.

In the event that an accidient occurs, the HSO will document any information related to the accident, and the project manager will use the information to complete an ateidest report to inelude notifiteation of the Subcontractor Safery Group at 4-5663. Follow-up axtion will be taken to correct the sithration that caused the aceident.

- In the event that any member of the field crew experiences any adverse effrects or symptoms of exposure while on the seene, the entire field erew will inmediately balt work, potify the HSO, and act according to the instuctions provided.

- The discovery of any condition that would suggest the existence of a situation more hatardous thar anticipated will result tin the evacuation of the field tean and re-evahuation of the harard and the level of protection required.

- In life-threatening situations, work strail stop and care will be instituted immediately. All personnel will leave the affected area. Respisators will be removed when clear of the affected area if breathing difficulties are ovidest. Prorective cloting will be temoved or cut awry if this will not canse delays, intertite with treatuent, or aggravate the problem. If contaninated

- If protective cloting canrot be rentoved, viction will be wrapped in clean materials, if conditions allow, to bito prevent possible contamination of modical personnel and ambulinges.

- In the event that an accident occurs, the HSO will folly docamentt all pertinent informatiog retated to the accident An Employee Initury/Exposure incident Report form is included in Appendix C, and this form will assist the HSO in documenting important details. This report will be provided to the project manager upon rog̣ust.

- Notification will be made by the SRS RU PM (or designtet)to the ERD SIRIM Reporting Official and SRSOC for emergeney medieal seryiets. Please rofer to WSRC 9B, "Site frem Reportahility atio: issue Management (SIRIM)" and WiRC 9B1, "General Site Requirentivits for SIRIM" for informiătion regarding emergency reportinfsas it relates to STRIM.

Rechearsols of the epgergency procedures shal te conducted by the HSO if warranted 


\subsection{Personal İjury}

Designated work site personnel will be trined in Ameriean Red Cross (or equivalent) finst aid procedures and witl administer appropriate first aid treatmont, incluoing CPR, in emetgency situmtions. These general emergency procedures will be carried out in the event of injuty:

- Notify the HSO of the incident.

- If the victim ean be moved stafely, remove the victim firor the Exelusion Zone to the Decontamination Zone using cosablished control points.

- Administer first aid as appropriate.

- Notify SRS Operations Center (SRSOC) @ \$-2117, 5-1911, 5-2661 or CB radio, channel 9, to request entergetscy medical serviess.

NOTE: The unit HSO and RCO personnel (if in a jadiological area) shall ditert the removal of infured persomel from the Exclusion Zone and shall approve any necessary deviation from establikhed decontamination procentures. Sucb deviarlon stall be based upon the severity or tife threatering natme of the injery.

\section{Chemical Exposure}

This procedure shall be followed for chemiteal exposure emengencies:

1. Move the victim from the immediate ara of exposure or contanination only if absolutely necessary, taking precautions to prevent additional exposure of other individuals.

2. Notify the HSO of the exposure incident.

3. If victim can be moved safely, proceed from the Exclusion Zone to the Contamination Reduetion Zone through established control points.

4. Decontaminate clothing or remove if safe to do so.

5. Adminitter adffitional fixst aid treatment as appropriate.

For skin or eys contict, thoroughly wash affected area with water (eyes should be flusthed for at least 15 mimules).

Eor inhalation exposent, ensure that viction bas adequate fresb aif.

Call SRSOC @ 5-2117, 5-1911, 5-2661 or CB ratio, channet 9, to request emergency medied sevitet.

\section{$11.4 \quad$ Firt or Explosion}

In the event of a fire of axplosion:

Immediately evacuate thjured personnel and leave the area.

Administer first aid to apprepriate.

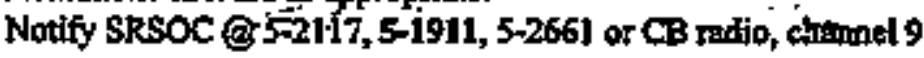

\subsection{Emergency Coutocts}

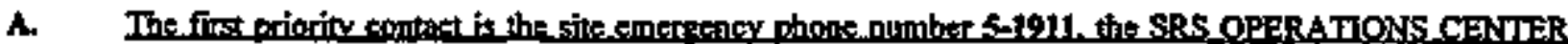
(SRSOCC. which will contact ambulance and other conergency heilp as otevessary. 


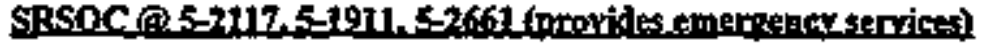

NOTE: In the event no telephoote can be reached, Chamel 9 can be accossed with a Citizens' Bind (CB) Radio for emergency assistance.

B. The following people are designated as Site ltem Reportabjity and lasue Management (STRIM) Reporting Officials (SRO) in the Waste Site Contingency Plan located in the RCRA Part A Permit. These peopie have the authority and resources necessary to deal with a waste sire inxideat. An ERD SRO is to be contanted ff an tacident occurs.

Tabe 3

ERD Phone Listing

\begin{tabular}{|c|c|c|c|}
\hline NAME & DEPAFTMETT & SYrE PIONE & FOME PHONE \\
\hline $\begin{array}{l}\text { Primary: } \\
\text { Kevin J. Kuelske }\end{array}$ & ERD & (805) 644-6659 & 1706 855-0766 \\
\hline $\begin{array}{l}\text { Secondary: } \\
\text { Wayne F+ Jolmnson }\end{array}$ & $\mathrm{ERD}$ & $\begin{array}{l}(803) 644-6858 \\
\text { 725-PAGE (2038) }\end{array}$ & $(803) 278-3689$ \\
\hline
\end{tabular}

Notify the ERD if unforeseen circumstances roguive the immediate proturement of additional personal protective of emergency equipment

Attending emergency physicians should be given the tekphone number of the sebcontractor's medical direator to obtaia immediate access to an employes's medieal records and for cionsulintion puposes.

\subsection{Accident Reporting}

On-she-job accidents or insiderts nesulining in injwy or thiness to personnel will be reported to the ERD project manager following any aceident. Any accidents in an RBA will be Ieported to RCO for their reporting requirenuthts. Subcontractors are required to follow injury/illness reporting regulations as specified in 29 CFR 1904 as well as contecting the Subcontractor Safety Group (Reuben Raysor (3 45663), The HSO is responsible for providing a withen report desinibing unsafe work practices, conditions, illness, infiry of timcidents for the WSRC RI PM to be used when filing a report with the ERD and the Safety Department in arcortince with SIRM. Please . refer to WSRC 9B and WSRC 9B1 for information regarding accidemt reporting as it retates to SRMM. The reports will contain the following informations

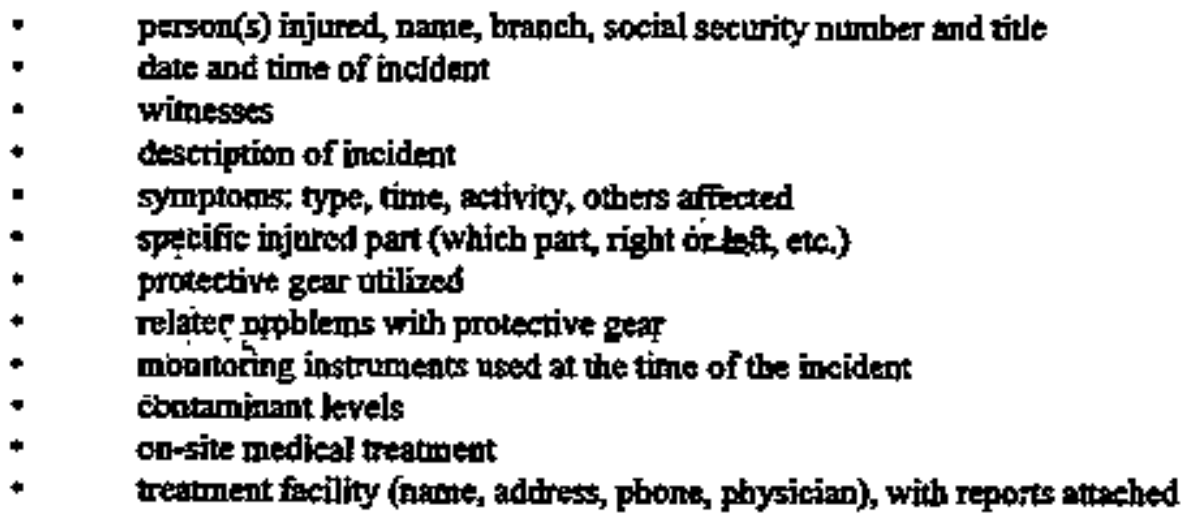


- acident investigation (canse, conrective action)

- summary of adequacy of the plan.

- report date, author signature, and title.

A sample Employe Injtry/Exposine Incident Report is included in Appendix C.

\subsection{CONFDNOD SPACE ENTRY}

Site Procedures (Procedure 33 of WSRC 8C) shall be followed, inchuding the issuance of a Woik Clearance Permit and a Confined Space Entry Permit. Refer to WSRC 8Q for more information. SRS-specifie training is requitred for confined space entry. No confined space entries are expected to be required for the investigation activities.

\subsection{SPLL CONTROL}

A spill control kit will be located at the Waste Unit at the diretion of the HSO, project marager, RCO or IH. If aid is required in containing a spill, contat SRSOC via radio (CB-9) or via telephone (5-2117, 5-1911, 5-2661).

All spills should be reported immediately to the waste site eustodian/project menager, Kovin J. Knelske (644-6659) and ERD Environmental Coordinator, Susan Dyer (644-6764). Spill reports will be made in accortance with the WSRC 9B Mannal.

Al dilling operntions will comply with spill control messures described in WSRC 3Q5, Section 3.0, WSRC 3Q Procedure 2.4, and WSRC 1B Manual. Any equipment remaining in one location for more than 1 work day is also subject to these requirements. A spill eleminip/control kit will be provided at the site.

\subsection{RECORD KEEPING}

All work clearance permits shall be maintained by the project manager and the HSO. Each subzontrattor dust maintain employee training records. A recond of al! significant evepts oceuring at the waste unit incheding visitors and safety operations will be maintained by the HSO. The HSO will complete the health and safey sheld forms (Appendix C) as necessary. The information to be logged will include:

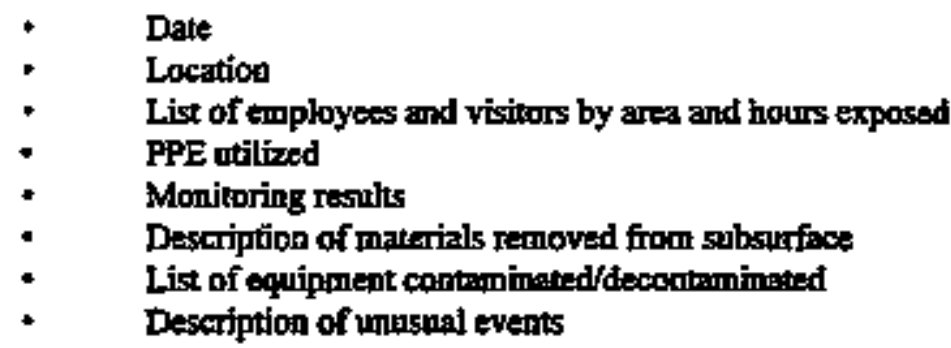

Other records to be mgintained by the ERD Projeat Engiucer or ER Trating include:

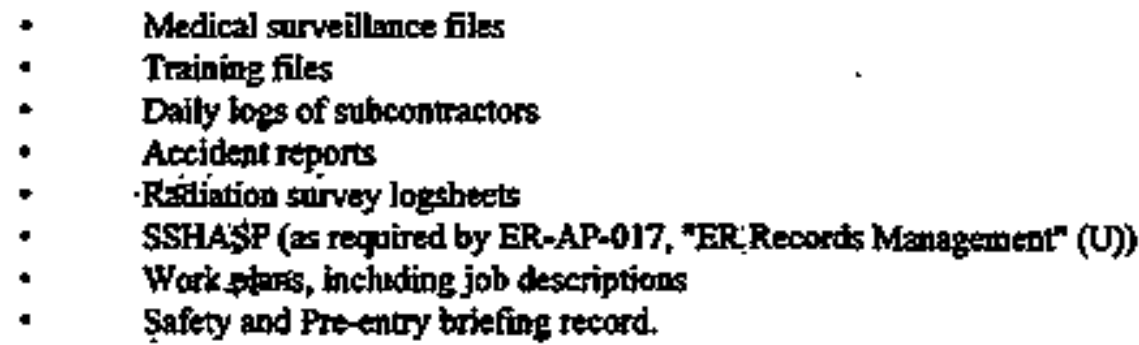




\subsection{RESERENCES}

\section{1-Relereaces}

1. Indutrial Fygienc Procedure Manual WSRC 40, "II - Heat Stress Managenent".

2. Titie 29, Code of Fecteral Regultions, Part 1910.129, "Hazardons Waste Operations and Emergency Response".

3. WSRC Procedure Manual 97, "Site lien Reportabjlity aud lssue Management (STDM)"

4. WSRC Procedure Mapusl 9B1, "Geveral Site Requiirements for SIRDM"

5. WSRC Procedure Manual 305, " Fydrogeologic Data Collectiop".

6. WSRC Procedure Manual 40, "Irductrial Hygleat Manual".

7. WSRC Procedure Manual SO, "Rediologicol Controts Manual".

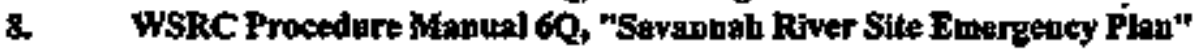

9. WSRC Procedare Mapural 80, "Smployee Safety Mabual".

10. WSRC Procedare Mavual 20Q, "Health and Sofety Mabual for Hazsidous Waste Operations".

11. WSRC Proeedure Manual WSRC-C1, "Enviroumental Restoration Administrative Procednres Manual (U)".

12. Phase If Revision I Renedial Investigation Work Plob for the F-Ares Retention Basin (182-3F) (U), October 1994, WSRC-RP-94-498.

\subsection{Appendices}

Appendix A: Summary of Operation Specific Safezy Procedures

Appendix B: Guidelines for Heat Stress and Cold Exposure

Appendix C: Suhcontractor Technical Dversight/HSO Heath and Safety Forms 
SUMMARY OF OPERATION SPECIFTC SAFETY PROCEDURES

\section{E-39}




\section{SUMMARY OF OPERATION SPECIFIC SAFETY PROCEDURES}

\section{AI MONITORING}

Al.1 A meniber of RCO will coordinate and conduet moettoring of radiosctive specties. Periodic examination of materials removed from the surface and subsurface will be conducted by RCO to determine if respiratory protection or other PPE is required.

A1.2 Monitoring with an OVA of cquivalent before and during drilting will be perforned by the HSO and/or IH as netssary, to determine if respiratory protection is required as described in seetion 3.2.3. Any instruments may become radiologically controlled if they are contamtinated during their use th the fietd.

\section{A2 PROTECTIVE CLOTHING}

A2.1 Standard safety clothing (Lovel D) will be required wless monttorimg indicates otherwise. Personitel who witl be in contast with waste matertals removed from the waste site will have chemical resistant coteralls, shoe covers, and gloves or appropriate radiation protective cloching as circumstances require.

A2.2 Decontemtnation of clothing is not antitipated to be necessary. If monttoing indicates otherwise, the HSO will provide directions. In cases where contanination is limited, disposable coveralls, gloves, and shoe covers are recommended.

A2.3 SCBA is to be used in emergency conditions ooly. Work should halt imtit monitoring indicates SCBA is no longer necessary.

\section{A3 OTHER CONTROLS}

A3.J At the direction of the HSO, SCBA or an equivalent portable eroergency at supply should be located within 150 feet, or as close to the RCA as practical, of active tijling locations for rescue purposes. At least I person qualified to use SCBA should be available during drilling operations.

A32 At least one persen nomally located ontside of the exclusion zone should be trained in CPR.

A3.3 Generators stould be located downwind of drilting depending upon the air activity.

\section{A4 DECONTAMINATTON}

Contamtinated equipotent and contraminated PPE camnot leave the waste site.

Equipment

A4.] The auger flights will be stean cleaned between each bole. HSO is to verify that the auger flights have been properly cleaned prior to beginning drilling at a net. site. The HSO will ensure that the split spobns are also decoptaminated afjer each use: Runoff will be directed onts the ground and away from holes is the waste site.

A4.2 Before the ofrill rig can move to a new work area, the HSO and RCO must give approval that the work area is ckosed as ciesn. 


\section{PeE}

A4.3 Workers exiting the exclusion area will be mogitored with the OVA or equivalent to determise if contamination is present

If no contamination is detetet, the PPE should be deposited in the support zone. Relse is encouraged when possible. If contamination is detected, decontaminate as cessribed in Section 10. If PPE cannot be decontaminated, it must be placed with the waste material removed from the hole. Successfully decontaminated PPE may be placed in the support zone and reased when teasible.

\section{AS CONTAMDNATED WASTE MATERIALS}

Contaminated waste materials are not expected to be geperated in large quantities al this waste unit.

A5.1 Contaninated PPE, soil, and waste from holes will be stackpiled within the waste site and covered at the end of exch day with plastic or tarps. Scockpiles should be placed and covered so that runofi will not cany any of the materials from the stockpile location(s).

A5.2 Contaminated PPE will be placed to the side of the work site as directed by the ERD Project Manager.

\section{A6 CONTINGENCY PROCEDURES}

A6.1 In the event that an atruosphere $>10 \%$ of the Lower Explosive Limit (LEL) is detected, the area is to be evacuated and equipment is to be shutdown until levels go below $10 \%$ LEL.

A6.2 Section 8 of the SSHASP describes emergency procedures. Phone numbers are listed in 8.5 . Ctamel 9 of the Citizens' Band radio is monitored by energency response persomel.

A6.3 If an atmosphere $>50 \%$ of the LEL and/or $<15 \% \mathrm{O}_{2}$ persists in a hake, then the hole will be vented by introducing a gas into the bottom of the hole via a fiexible hose. Air may be used if the mixture is below the Upper Explosive Limit (UEL). An timert gas such as nitrogen or argon is preferred.

\section{A7 EXCAVATION}

No excavation is anticipared as part of this project.

Procedure is to be devetoped by subcontractor performing excavation and will comply with guidetines set forth in this SSHASP and OSHA Regulations 29 CFR Part 1926, Subpart P "Excavations." 
Irdestrid Fyolians Nincuat

Minged:

Procedirta:

Effective:

Hor Ror 1

Page:

6 risto3

1 of 13

Heat Stress Management

\section{Putpose}

\section{Scope}

The repons

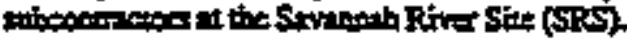

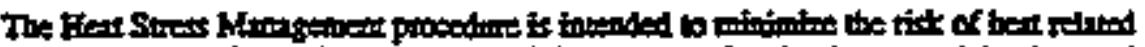

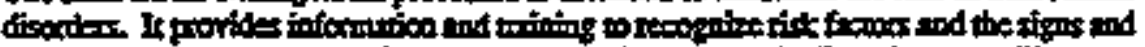

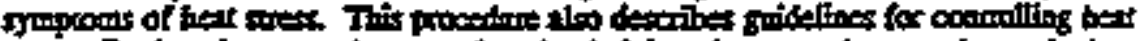

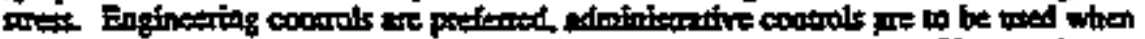

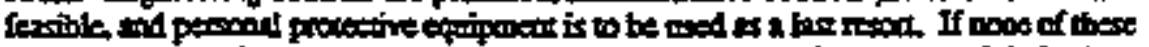

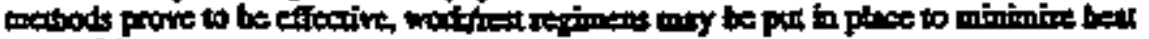
stres ritio.

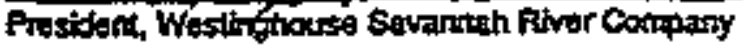

\section{Tems and Defintatons}

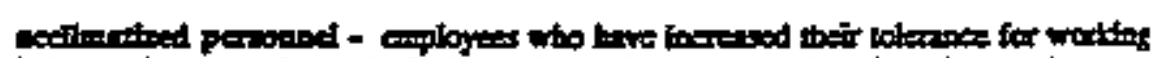

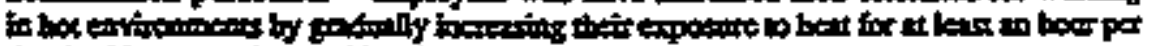

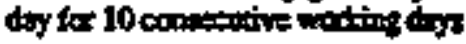

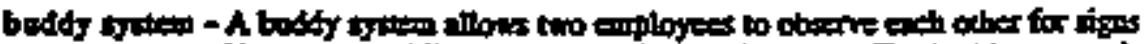

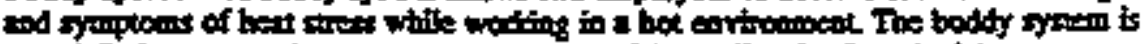

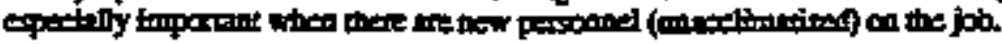

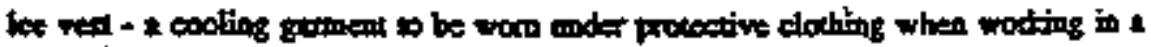
hoxestuoniert

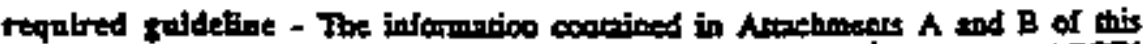

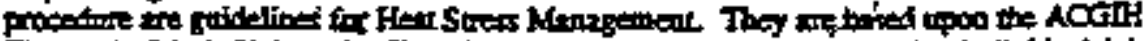

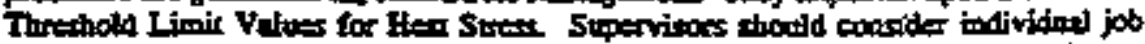

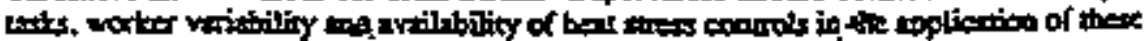

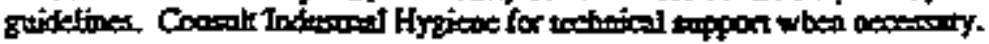

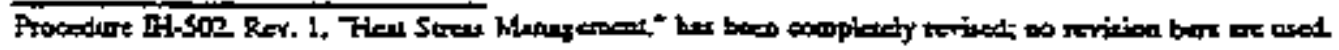


Mtenuat: Procedure: Ettective: Papa:
40 $1+502$, Fiev. 1 6rit/93 2에 13

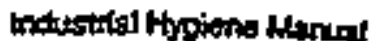

Hert Siross Aangemant

\section{Fesponstbtlities}

Mansgemeit

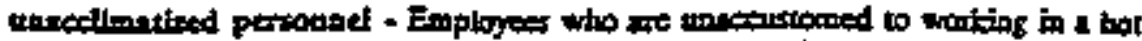

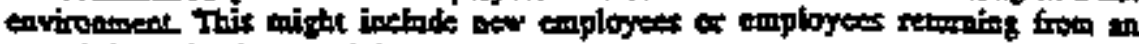
conded nerim (nowets)

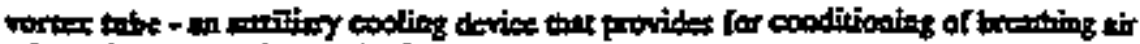

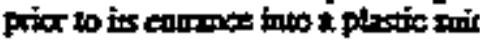

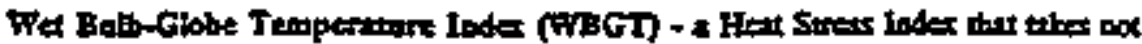

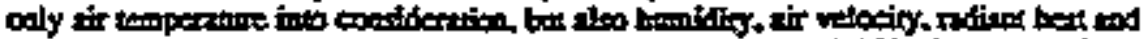

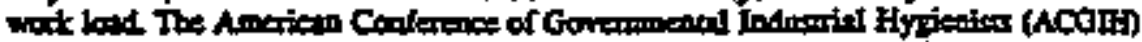

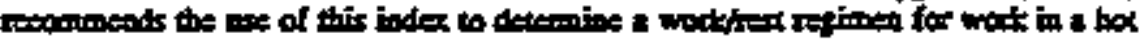

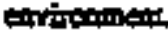

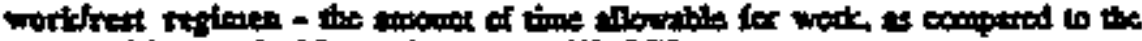

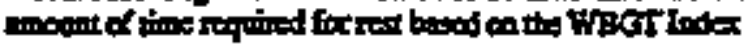

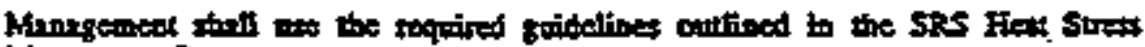

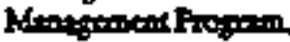

\section{Supervisors}

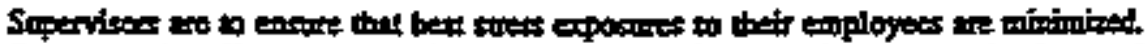

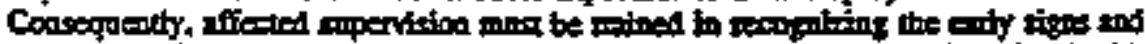

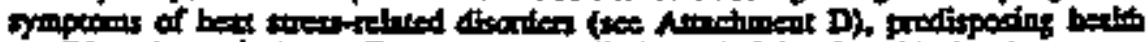

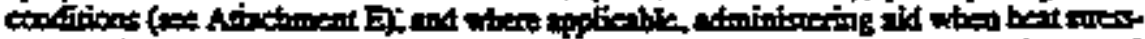

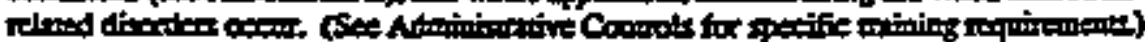

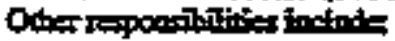

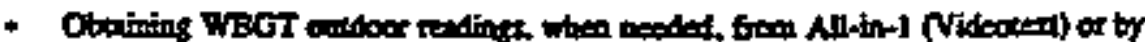

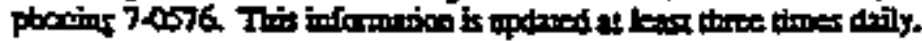

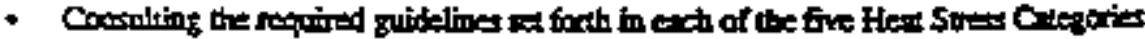

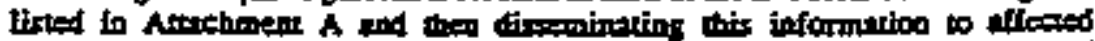

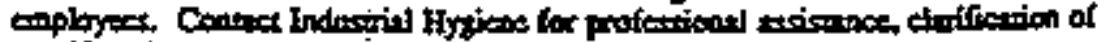

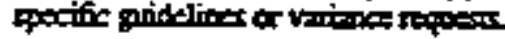

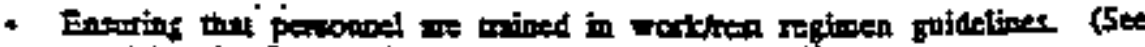

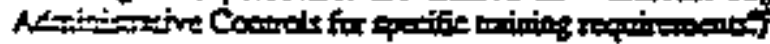

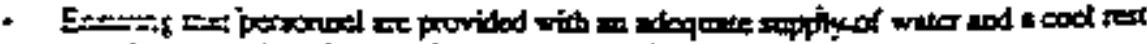

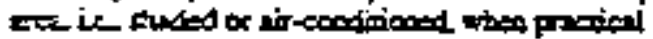

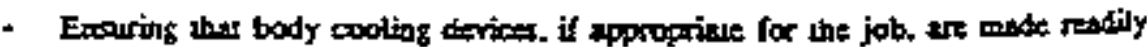

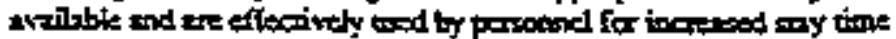


Wropet:

Procediure:

Efiective:

40

th.5\%2, Fev, 1

$6 / 1493$

Heat Stross Maragonant

Pane:

3 of to

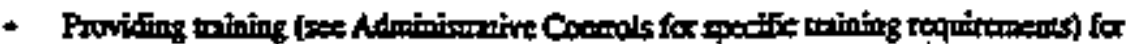

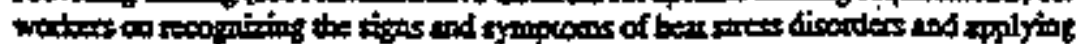

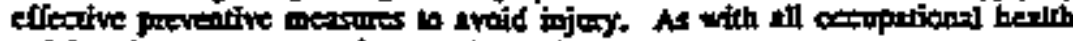
iniming, formel dormentation most be resined.

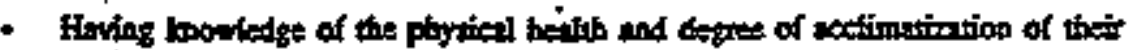
emplayos.

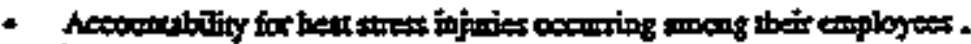

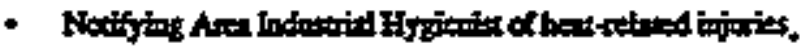

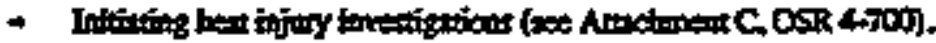

Inơustrial Hygiene

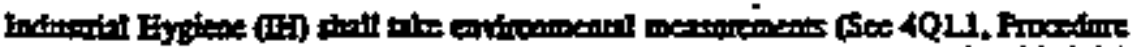

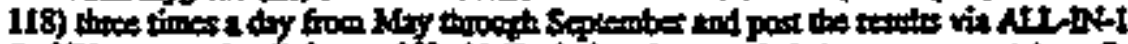

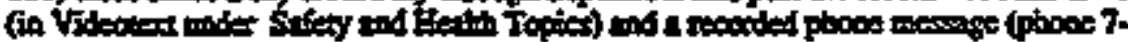

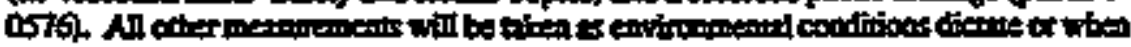

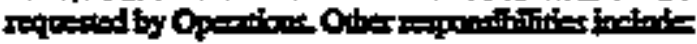

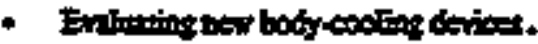

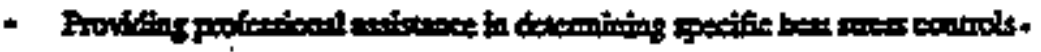

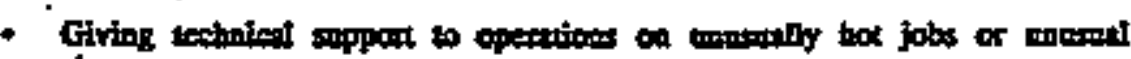
intorimases.

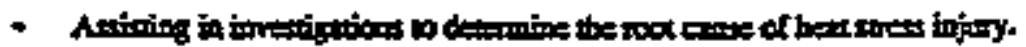

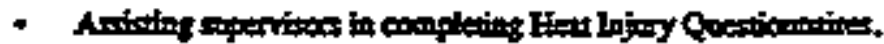

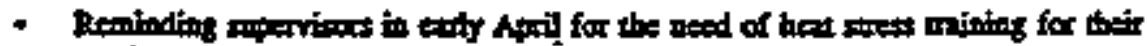
coplopes.

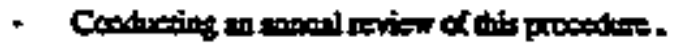

Health Protection Operations

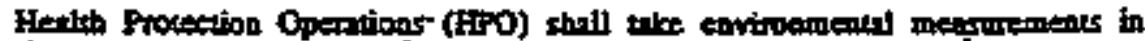

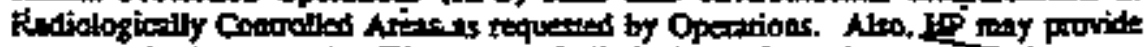

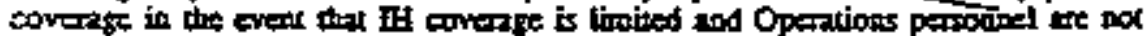
polifit. 
Manuat Orosedeste:

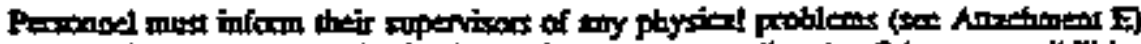

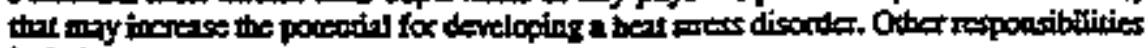
intors

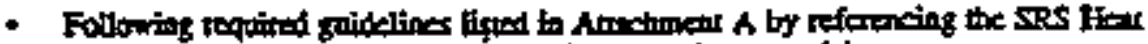

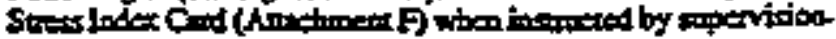

- Fupipang in the budty symen.

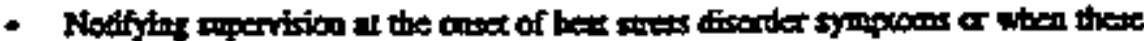

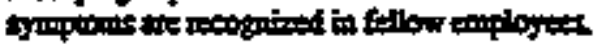

\section{Procedure}

\section{A. Heat Stress Categories/mplemantation}

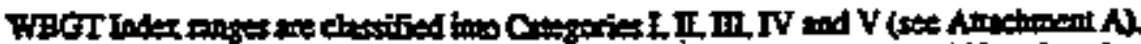

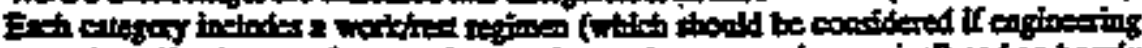

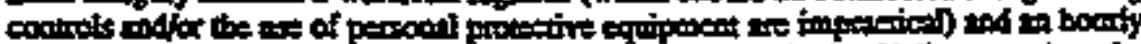

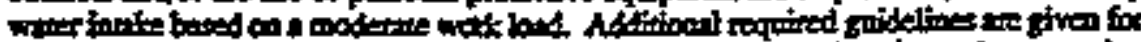

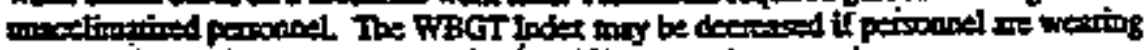

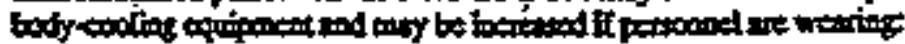

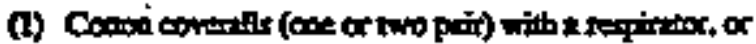

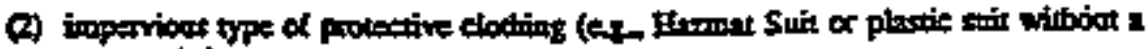
vates

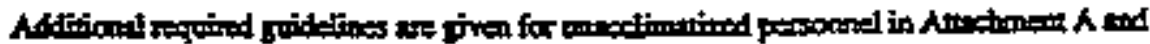
in Arratiment F.

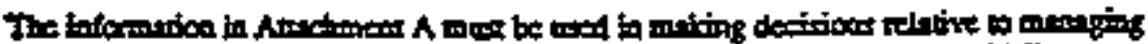

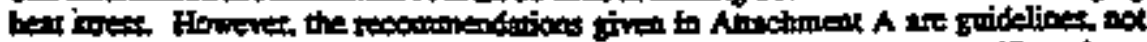

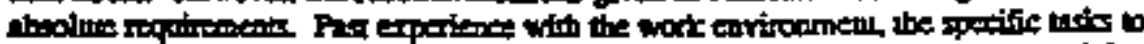

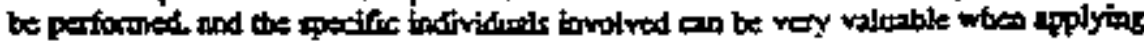

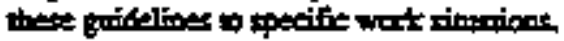

\section{Emvironmental Measurement}

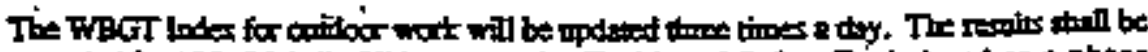

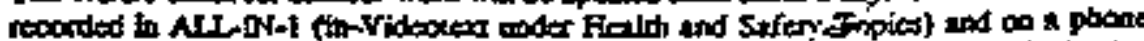

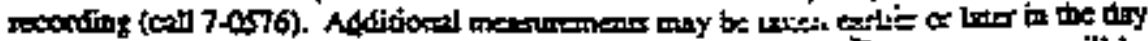

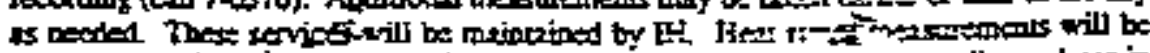

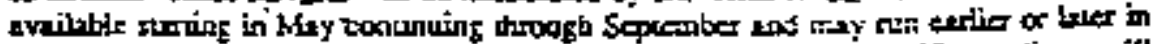

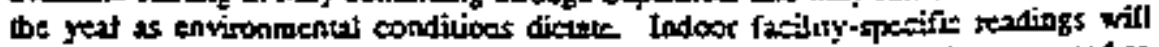

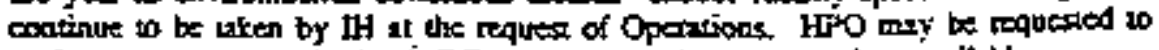

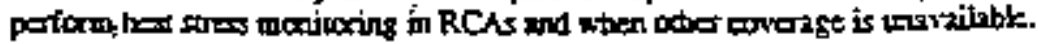




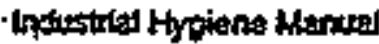

Nantist

Precedute: Hock Rev, 1

Etioctive:

6/14/83

Heat Stross Meniagomert

Pape:

5 of 13

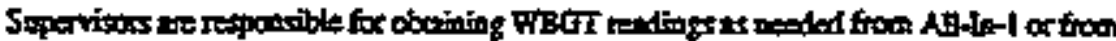

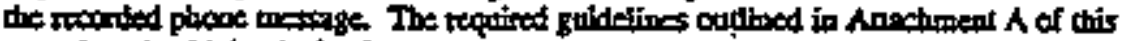

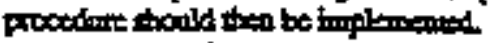

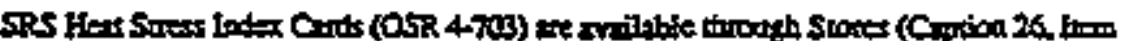

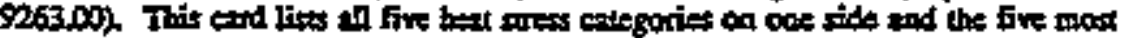

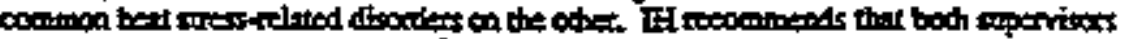

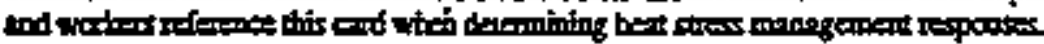

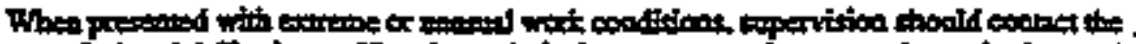

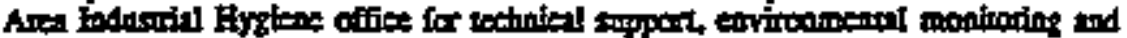

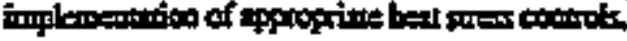

\section{Administrative Controls}

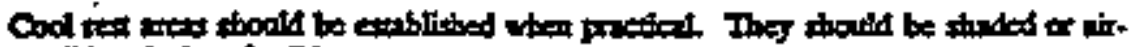

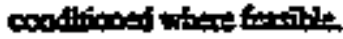

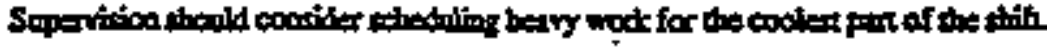

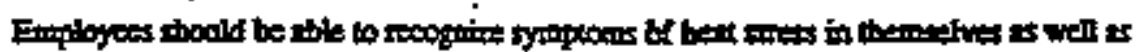

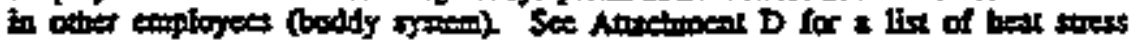
tymporent.

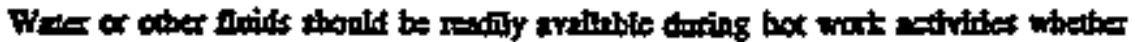

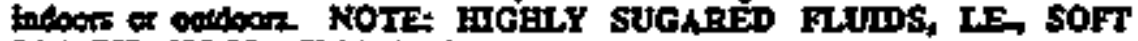

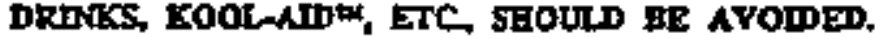

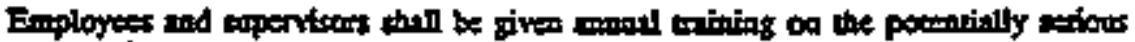

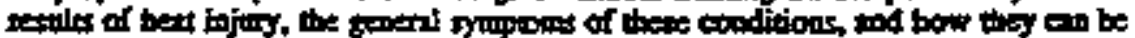

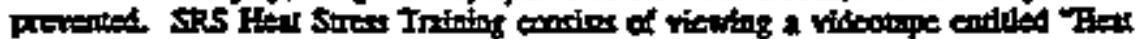

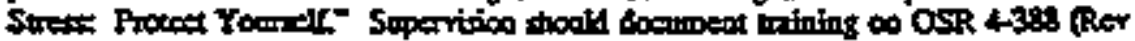

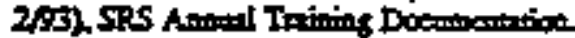

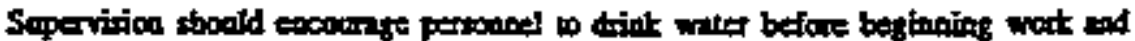

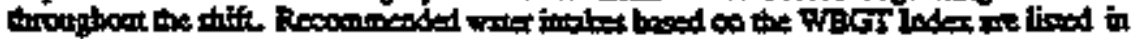

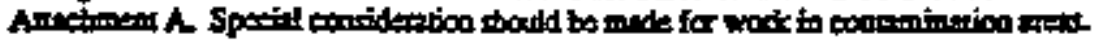

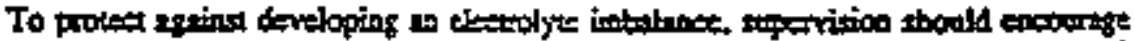

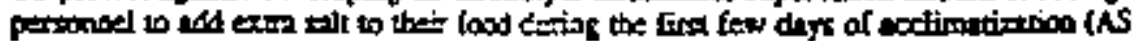
APPROVED BY A PFYSICDAN).

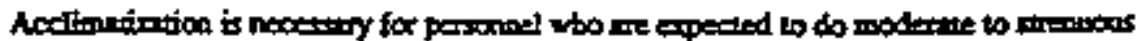

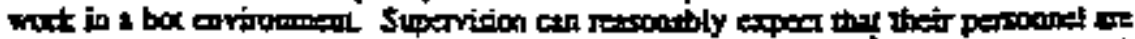

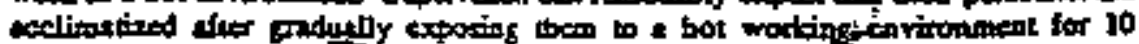

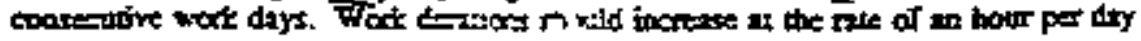
ouring this pariod

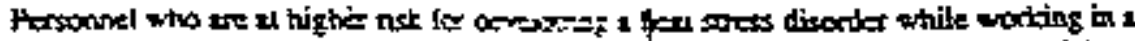

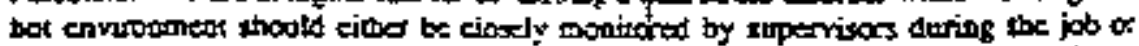

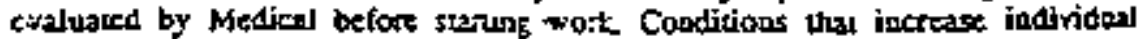

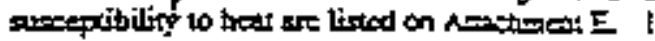


Martial: Procodutit: Etiective: Page:
40

HA-502, PLV. 1

E/14/93

6 of 13
Inclusital thyteng Maruas

Heat Stress Mangogmara

\section{Personal Protective Equipment}

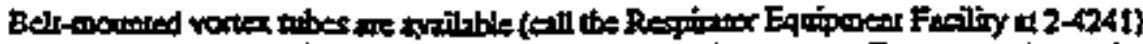

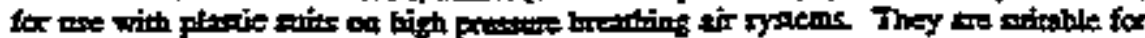

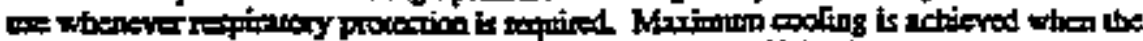

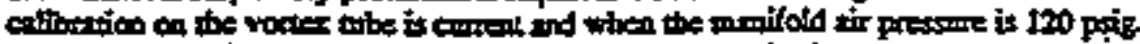

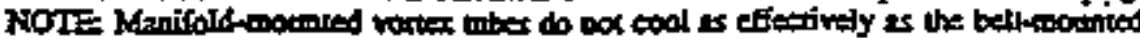
rimy.

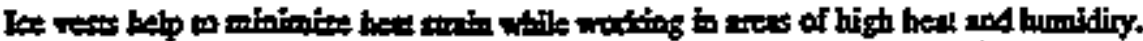

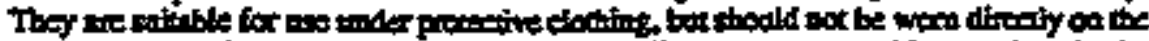

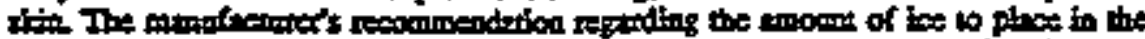

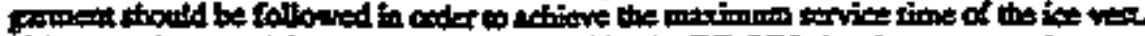

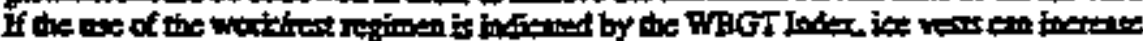

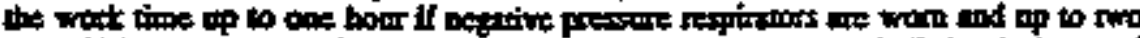

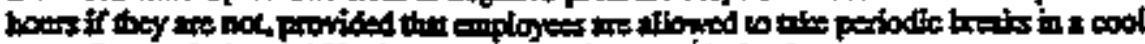

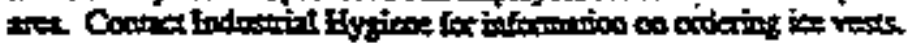

\section{E Engineering Controls}

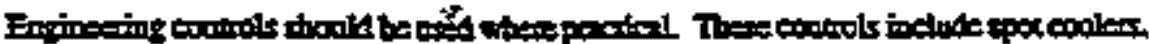

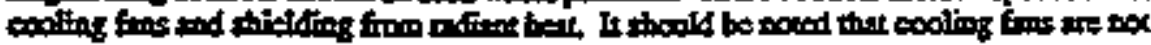

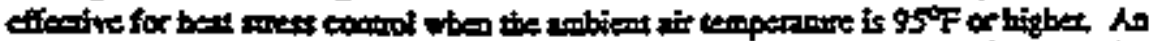

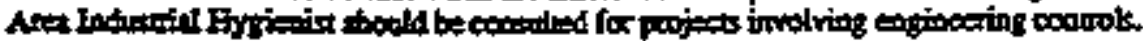

\section{F. Heat injury Investigations}

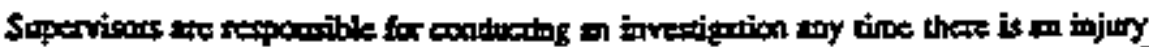

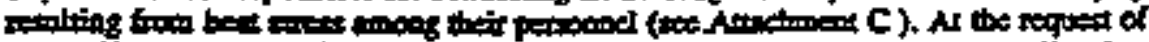

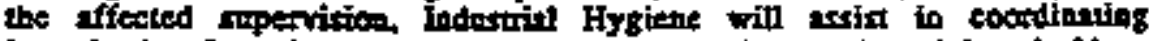

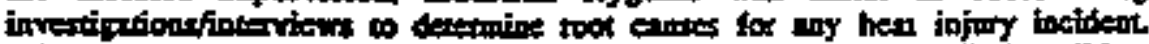

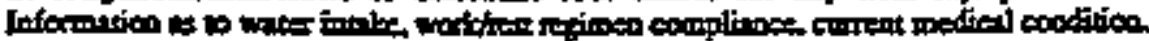

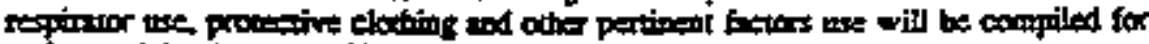

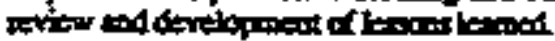

\section{Records}

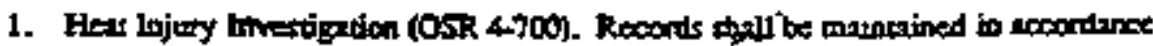

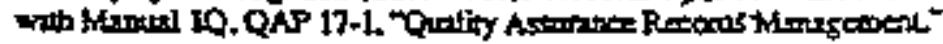


trocustrial Hygians Mtartat

HaAn Stress Maraqomeri
Kanual:

Prosediers:

Eflective:

Paga:
Hit502 Aor I

$6 / 14 / 93$

7 of 13

\section{Reforences}

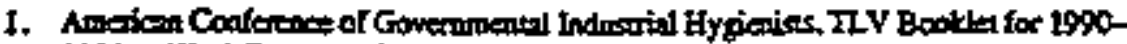
1991. WotkRer Regimes for a modente woth had was thed to develop Ancentent $A$.

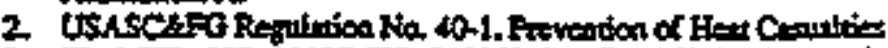

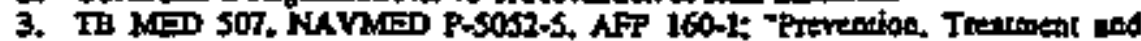
Contol of Hetriging

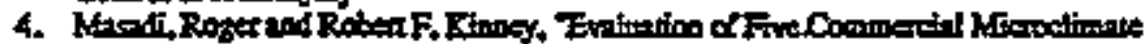

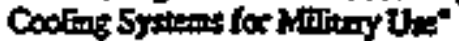

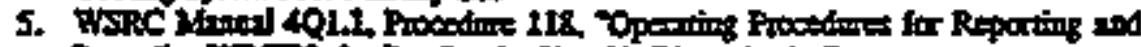

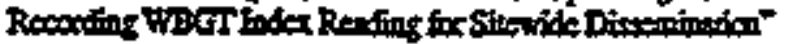

\section{Attachments}

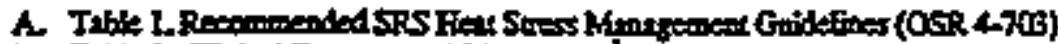

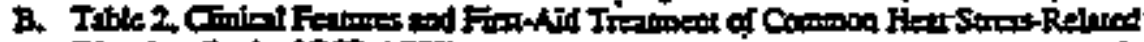

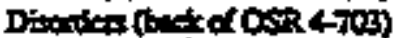

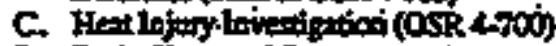

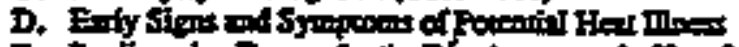

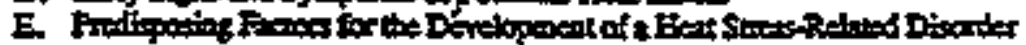

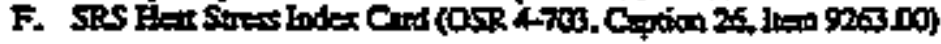




\begin{tabular}{|c|c|}
\hline 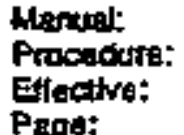 & $\begin{array}{l}40 \\
4+502, \text { Fiok, } 1 \\
61483 \\
8 \text { of } 13\end{array}$ \\
\hline
\end{tabular}

\section{Attachment A. Table T, Fecotnmended SRS Heat Stress Mankgetnent Guldellines}

\section{Table $*$}

Recommended SRS Hent Stress Management Guidelines

\begin{tabular}{|c|c|c|c|c|}
\hline CATreosy & 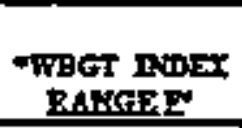 & 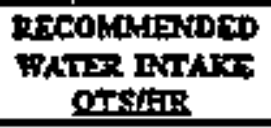 & 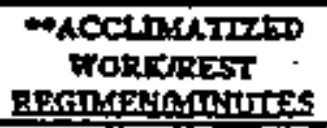 & 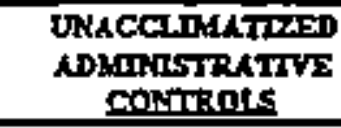 \\
\hline 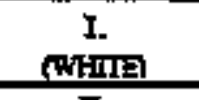 & $\pi 0-510^{\circ}$ & $1 \sqrt{2}$ & conimuocts & 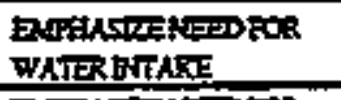 \\
\hline त् & $80-369$ & 17 & $4 S t: S$ & 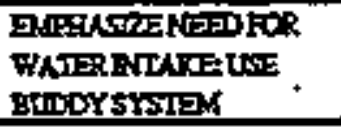 \\
\hline III & $500=\pi s$ & $\overrightarrow{1}$ & $30 / 30$ & 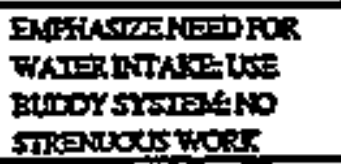 \\
\hline T. & $00-200$ & $1-12$ & $15 / \mathrm{s}$ & 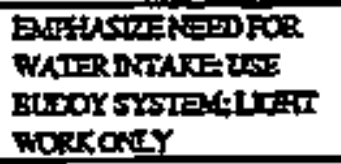 \\
\hline (Bun & 8000 & ORStar & 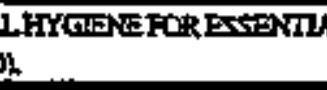 & LFORX WRHPOATHE \\
\hline
\end{tabular}

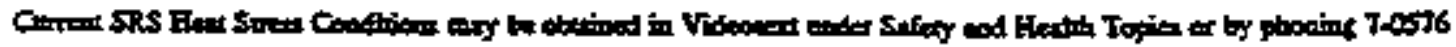

- USEC A

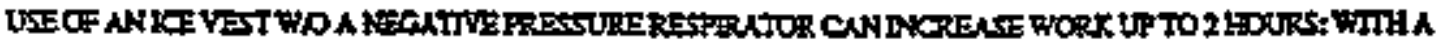
AEOATVEFREXXRERESTRATR UPTO1BOUR.

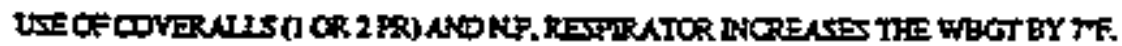

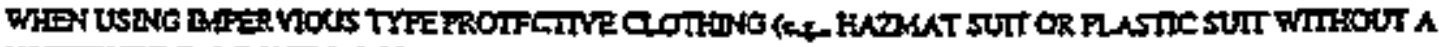

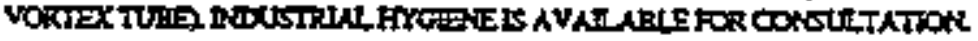

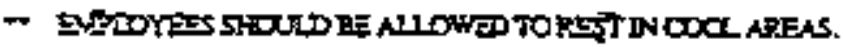


Indiectrial frtyotone Manutas

Hean Sress Marigemert:
Maruat:

Procedutra:

Effective:

Pata:
H. 500 Aors $6 \sqrt{4493}$

9 of 13

Attachment E. Table 2, Cilnical Fartures and First Ald Treatment of Common Heat Stress-Rielated Disorders

Table 2

Clinical Features and First Aid Treatment of Common Feat Stress-Reinted Disorders

\begin{tabular}{|c|c|c|}
\hline DISORDER & OAfICAL FEATURES & TR:ATMens \\
\hline GEAT SROKE & 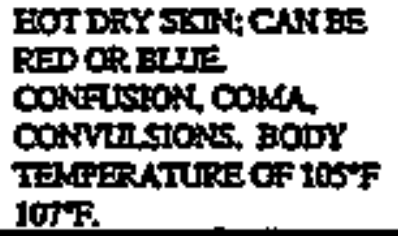 & 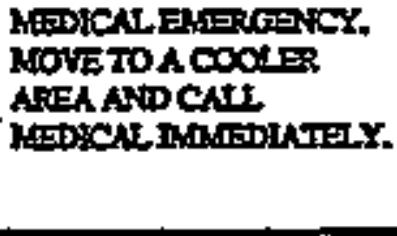 \\
\hline HFATSMCOEE & 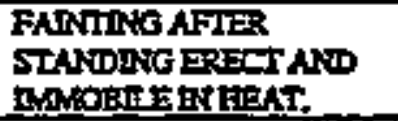 & 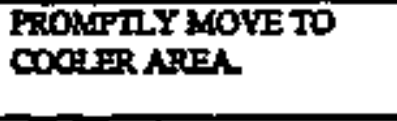 \\
\hline $\begin{array}{l}\text { ESTT } \\
\text { EXTALUSTION }\end{array}$ & 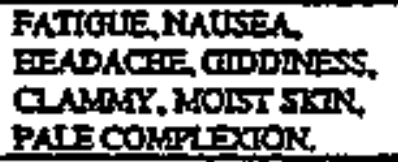 & 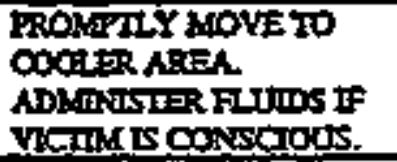 \\
\hline HrET QRAHPS & 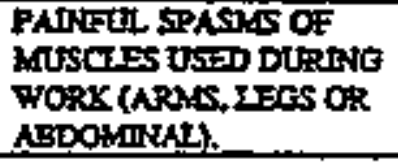 & 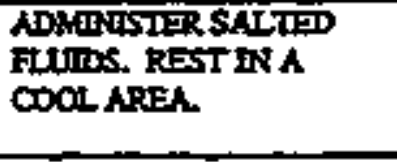 \\
\hline GEAT RASH & 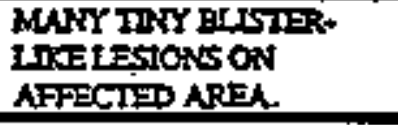 & 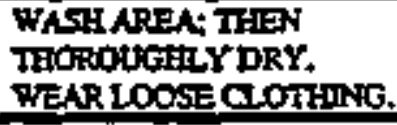 \\
\hline
\end{tabular}




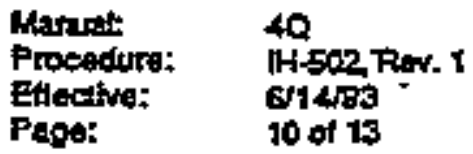

AttBchtrent C. Heat Injury linvestigation (OSR 4-700, Rev 3/10/83)

\section{Heat Injury Investigation}

Name

Date

Age Sex Orgenitrotion

Time of injury WBGT Rerding Locrtion

Stpervisor Address

Activity at time of tajury

Type of protective clothing worn tit time of iojury

Nutmber of cansecutive days on-job

Quantity of water consumed

Existing medieal conditions

Previous hest frjurg

Received ocoupationel heollth trating on beal stress?

Date of lest training

Diegnosis

Adữitiongl comments 
Intustrial Hoiane Manest

Hath Stress Manadomon
Mancent:

Propodura:

Etlectint:

Page:
IHste fitor. 1 $6 / 1493$

11 of 13

Aftachmant D. Early Signs and Symptoms of Potential Heat lisiness

- Thingy

- R-pid polks

- Eleatacis

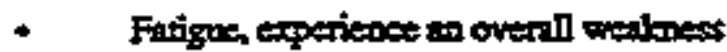

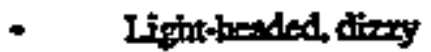

- Eligh body partare with or yithout swerting

- Pale elamiry sion

- Erathol faces

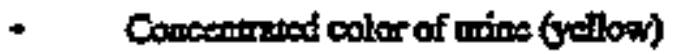




\begin{tabular}{|c|c|c|}
\hline 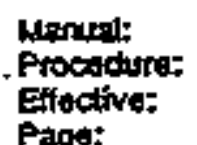 & 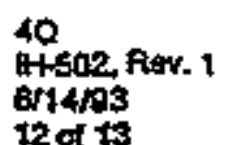 & 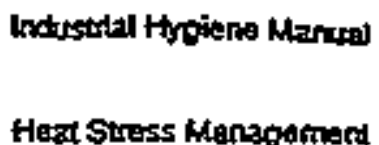 \\
\hline
\end{tabular}

Attachment E Predtsposing Factors for the Development of a Heat StrassRelated - Disorder

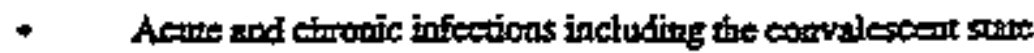

- Febialte (feveisi) conditioms

- Reactions to itrominizations

- Vaseniar diseness

- Confitions affecing sweat sectetion

- Stin ustrine such as hear rach or ecotre smbirm

- Provions cosimenos of heat injtity,

- Dehytrition

- Lack of alept cratlarive farigue

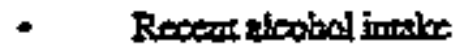

- Dieting.

- Sarne Inticarions. 


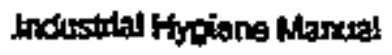

Hieat Suess Mangoenect
Manuil:

Frocedurt:

Elfective:

Page:
thi.50e 40 $6 / 14 / 93$
t3 of 13

\section{Attachment $F$. The SRS Heat Stress Index Card}

\begin{tabular}{|c|c|c|}
\hline 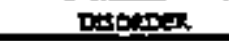 & 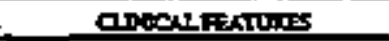 & THEXTOST \\
\hline artrsing & 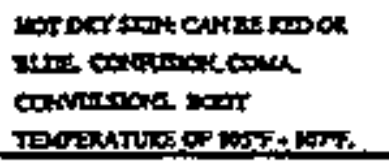 & 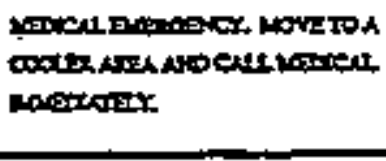 \\
\hline 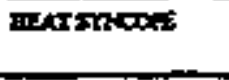 & 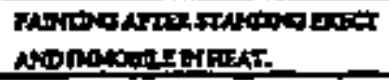 & 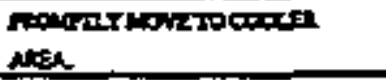 \\
\hline 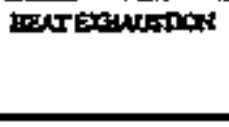 & 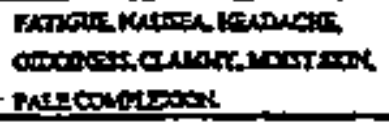 & 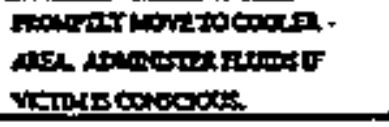 \\
\hline $\begin{array}{c}\text { protoutas } \\
\text {. }\end{array}$ & 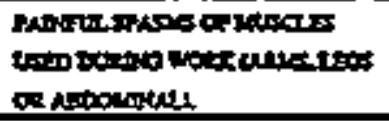 & 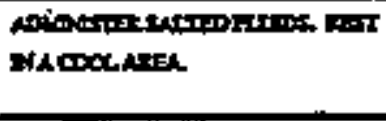 \\
\hline Exerest & 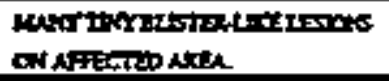 & 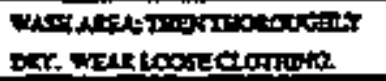 \\
\hline
\end{tabular}

\begin{tabular}{|c|c|c|c|c|}
\hline ClTERotiry & 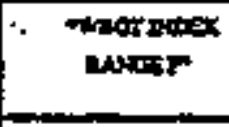 & 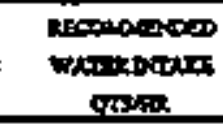 & 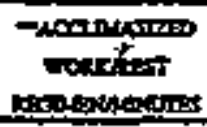 & 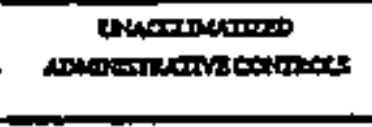 \\
\hline I & 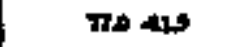 & in & concosuas & 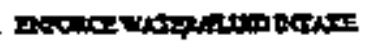 \\
\hline$\underline{-1}$ & Et+40 & in & sins & 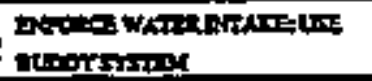 \\
\hline II & 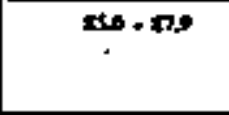 & 1 & saspo & 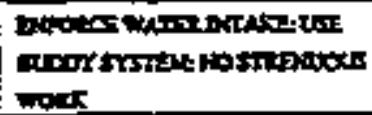 \\
\hline $\mathbf{N .}$ & 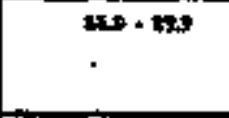 & $1-10$ & 13res & 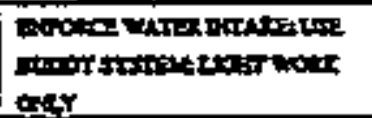 \\
\hline $\mathbf{v}$ & 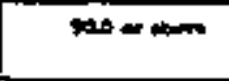 & 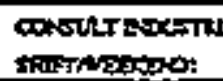 & I ponter & 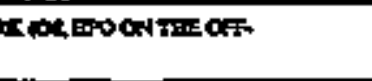 \\
\hline
\end{tabular}

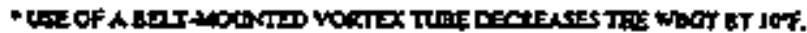

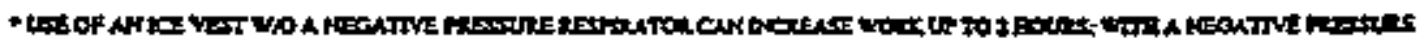

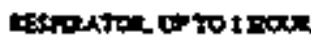

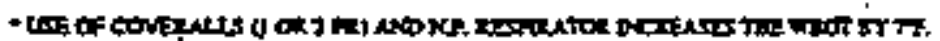

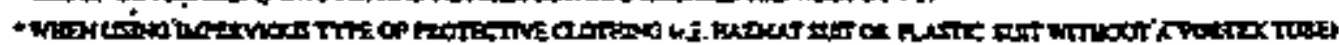

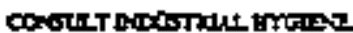

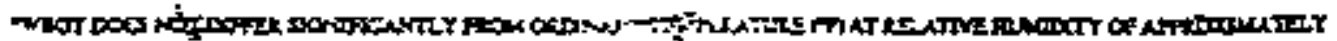

'rst on 0xestr.

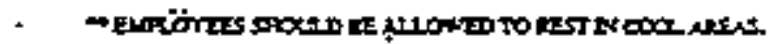

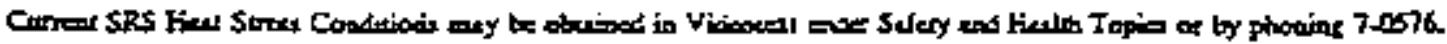




\section{COLD EXPOSURE PROTOCOL}

Cold injury (frostbite and hypothermia) and inopaired ability to work are dangers at low temperatures and when the wind-chill factor is fow. To guard against thers:

1. Do not allow cors oral cemperature to fatl below $968^{\circ} \mathrm{F}$.

2. Pain in extremities may be the first dangers to cold stess. Wokk must be terminated if pain and shivering becomes evident.

3. Change ciothes if worker becomes wet.

4. Follow Table 1 for determining Wind Chill

Follow Table 2 for Workwarm-Up Setiectute

\section{Clotbing}

Workers sball dress in layers protecting exposed skin. 'Layers should be shed as the air temperature rises.

6. Worixers in protective clothing should wear cotton or ofher absotteat materials to absorb swear and maintaio body warminth

\section{Buddy System}

All workers shill use the buddy system and consistently watch for frost-bit and/or exessive saivering.

\section{Warming}

If conditions warrant special teats, trailers or rest rooms, etc. will be made available for workers to warm in regular intervals. (Se Table 2.)

9. Dehydrafion

Warm sweet drinks and soups will be provided to provide caloric intake and fluid volurie.

10. The Health and Safety Ofticep

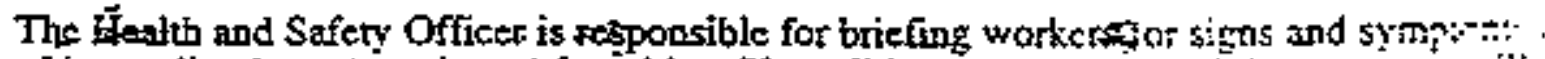
of impending hypothermia and frost-bive. If conditions warrant speciat themomet: wit! be considereo if the air umperatures falls below $60^{\circ} \mathrm{F}$. 
PROJECT:

PROJECT LOCATION:

PROJECT NO.:

HEALTH AND SAFETY OFFICER:

FIELD MODIFICATION TO SSHASP:

PREPARED BY:

TOHSO DATE.

ACCEPTED BY:

TOFM DATE

ACCEPTED BY:

APPROVED BY:

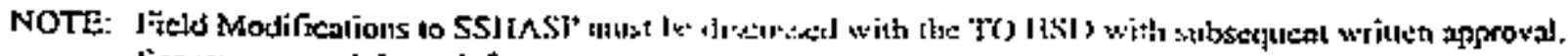
Secure approval throojalt tax 
SITE SAFETY MRETING ATTENDANCE

PAGE $\ldots$ OF

PROJECT:

PROIECT NUMBER:

MEETING CONDUCTED BY:
DATE:

TMME-

\section{SIGNATURE}

SIGNATURE DISCLOSURE: Each person, by signing this form, commits to baving read and understood; then accepts all requirements and procedurss outlined in the Site-Specific Safety and Health Plan (SSHASP.

\section{PERSONNEL PRESENT}

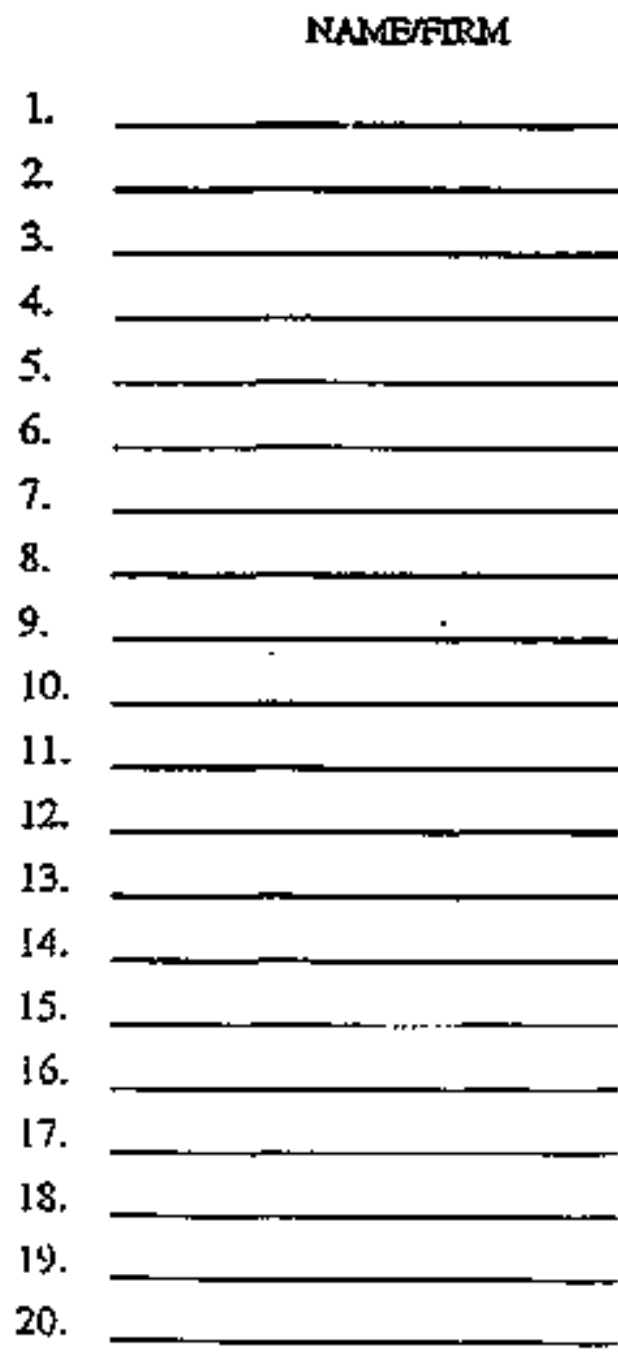

8.

9.

10.

11

12.

I4.

15 .

16.

14.

20.

SIGANTUREANTE

SOCIAI SECTIRITY NUMBER
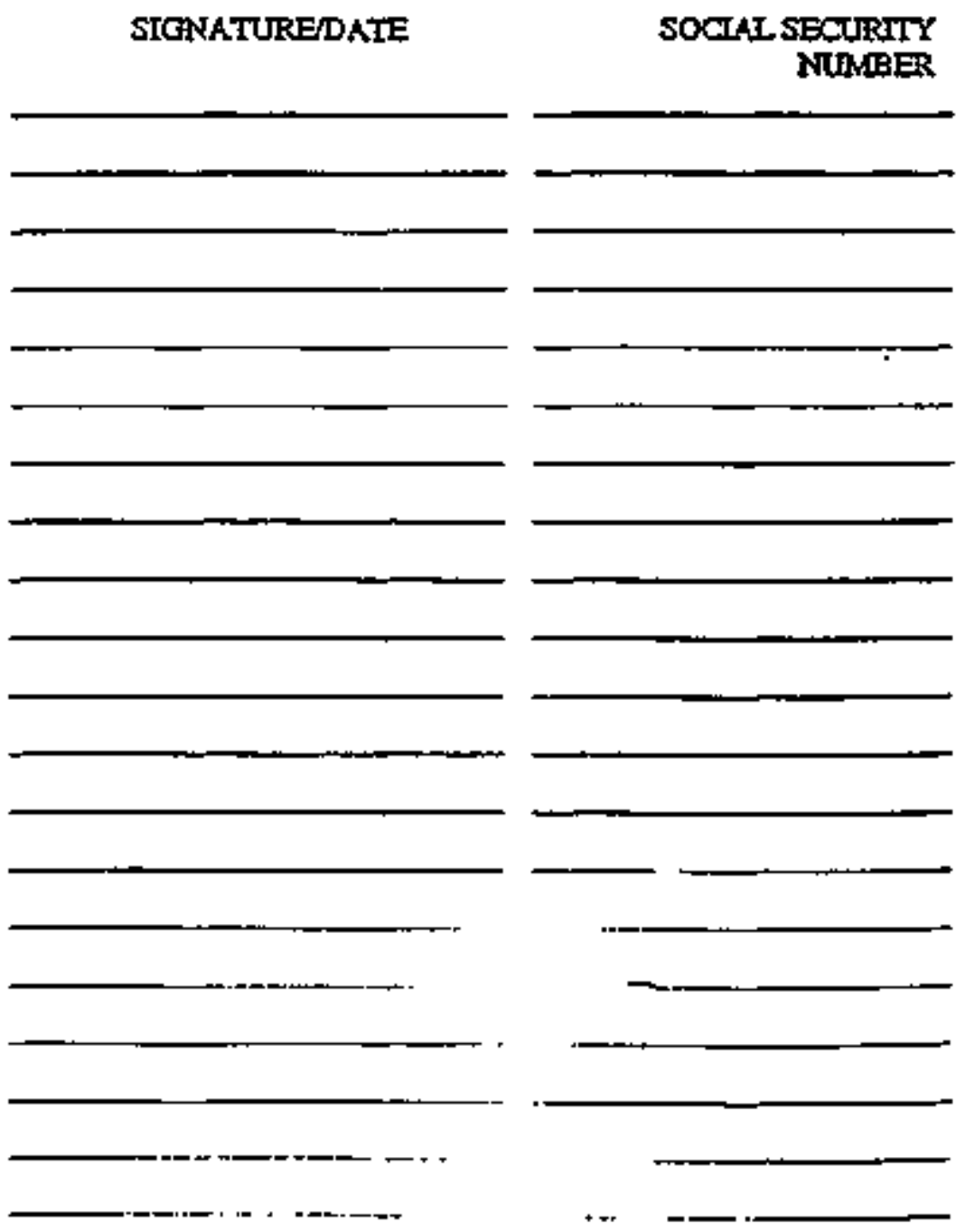
ATMOSPHERIC MONITORJNG LOG FIELD HEALTH AND SAFETY

Health and Safety Officer:

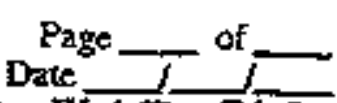

Circle; Stu Mon Tuc Wed Thu Fi Sat

Action Levels: D ' C ${ }^{\circ} B{ }^{\prime}$ ' (Stop work, call in for instructions)

(check box and write in levels for upgrade)

Task/Equipment:

Weacher:

\begin{tabular}{|c|c|c|c|c|c|}
\hline Tiroe & $\begin{array}{l}\text { OVA } \\
\text { PPM }\end{array}$ & $\begin{array}{l}\text { DUST } \\
\mathrm{m}^{\prime} / \mathrm{m}^{3}\end{array}$ & RAD & $\begin{array}{c}\text { NOISE } \\
\mathrm{dB}\end{array}$ & $\begin{array}{l}\text { Cornrents: Duration of Readings, } \\
\text { Upgrades, Location, etc. }\end{array}$ \\
\hline & & & & & \\
\hline & & & & & \\
\hline & & & & & \\
\hline & & & & & \\
\hline & & & & & \\
\hline & & & & & \\
\hline & & & & & \\
\hline & & & & & \\
\hline & & & & & \\
\hline & & & & & \\
\hline & & & & & \\
\hline & & & & & \\
\hline & & & & & \\
\hline & & & & & \\
\hline & & & & & \\
\hline & & & & & \\
\hline & & & & & \\
\hline & & & & & \\
\hline
\end{tabular}

Additional Comments:

Sintuinture: 


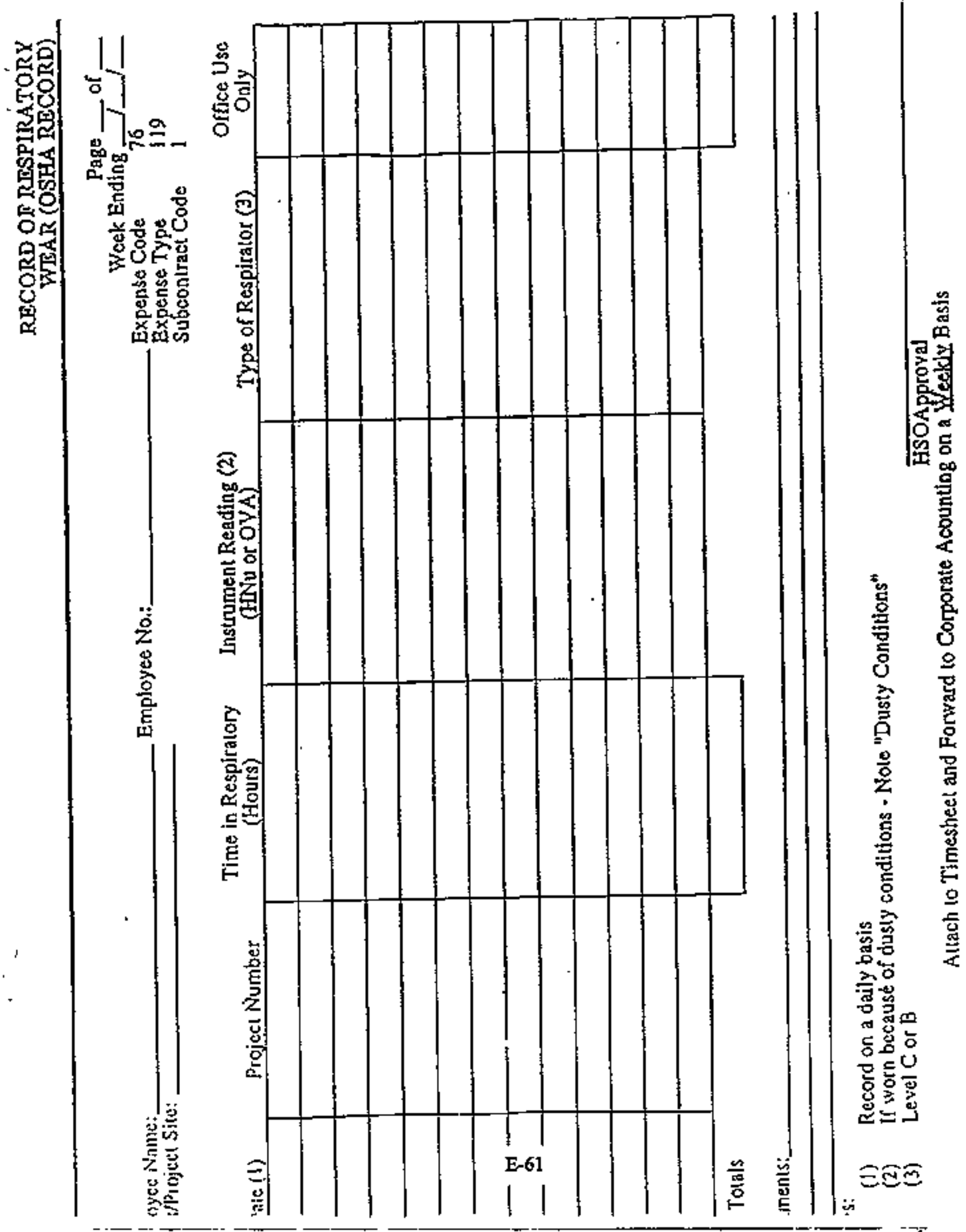


Date:

Employes: Employe No.

Site Name:

Sìte Location:

Project No:

Expostire (yes or no): Injury (yes or no)

Date of Incident: Time:

Location:

Activity During Time of Incident:

Site Conditions at Time of incident:

Temperazure

Wind Speed

Humidity

Other

Precipitation

Cause of Exposurellijury:

Type of Exposure/njury:

Matcrial Exposed 10 and Concentration Levels: 
Nature of Exposure/injury (part of body, etc.):

Hospital and Atrending Pbysician:

Medical Care Received (onsite and offsite):

Other Individuals Involved:

Winesses:

Has HSD Been Notified: No Yes (Anach Documenation) Incident Repart Completed by: 


\section{DAILY SIGN-IN/OUT LOG}

PROJECT:

PROJECT LOCATION:

PROJECTNO.

HSO:

DATE-

PAGE NOA:

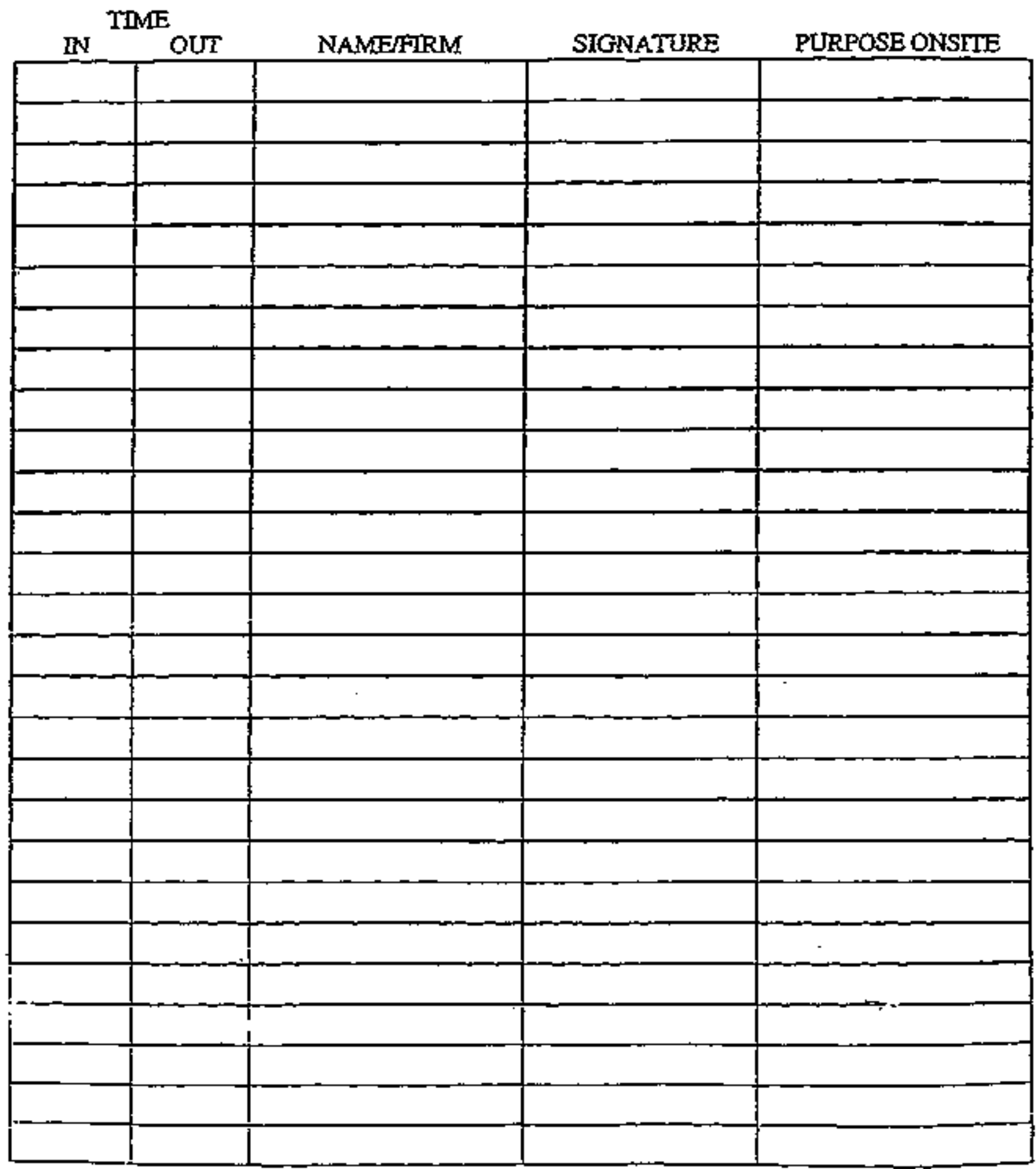

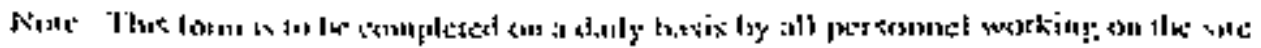

\section{E-64}


WORK ZONE ENTRY AND EXIT LOG (JOB EXPOSORE REPORT)

PROJECT:

PROJECT NOA-

STTE LOCATION

HSO:

DATE:

TME

IN OUT NAMESFIRM
FINU/OVA READINGS LEVEL PPE

SIGNATURE

BREATHING

ZONE)

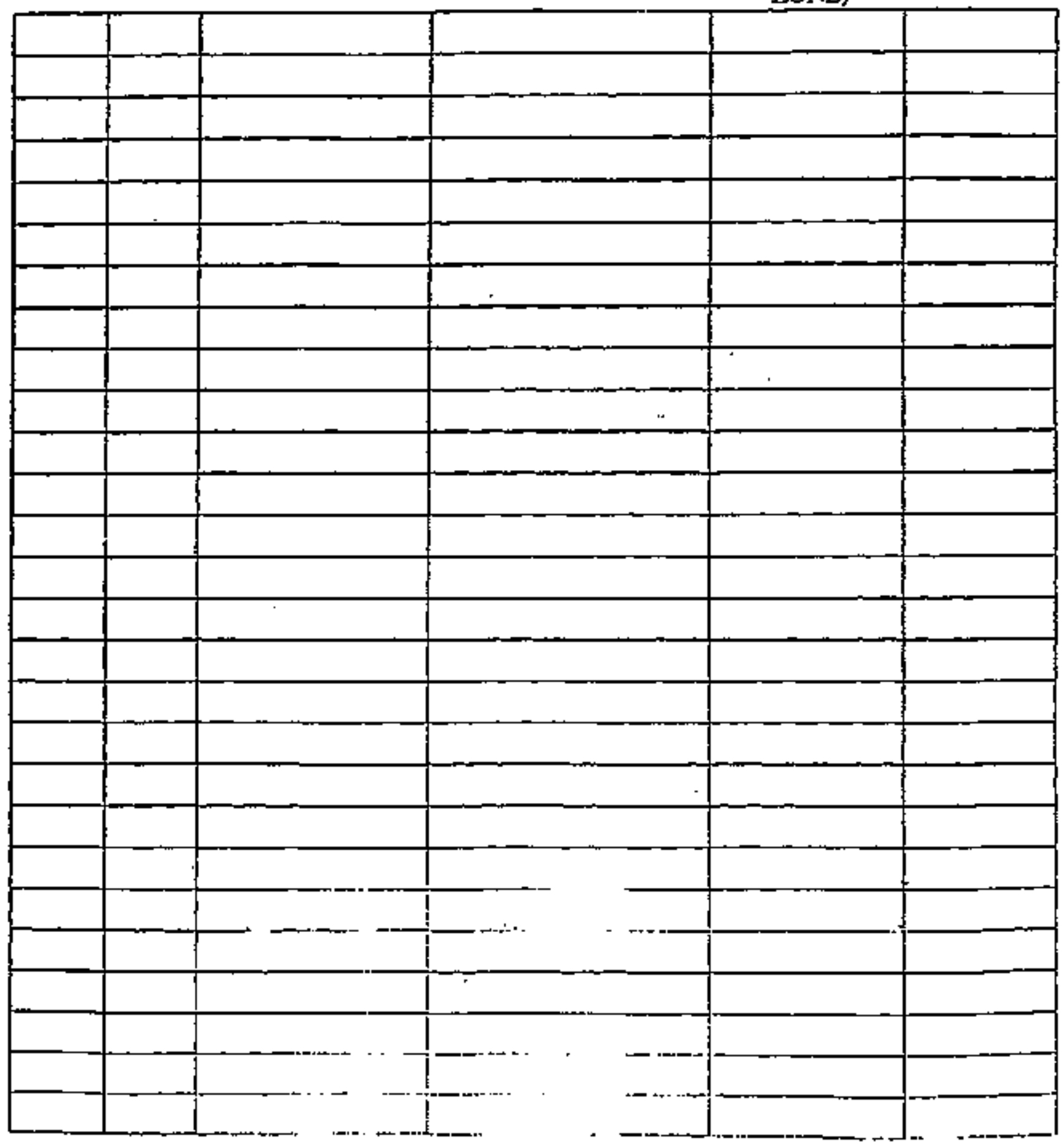


This Page Intentionaly Left Blank 


\section{Distribution}

\section{Federal Agencies}

3 U.S. Department of Energy Office of Technology Development Attn: J. Paladino, EM-521 Cloverleaf Center 19901 Germantown Rd. Germantown, MD 20874-12901

1 U.S. Department of Energy Albuquerque Operations Office ETN

Attn: Dennis Olona

Pennsylvaria/H Street

Albuquexque, NM 87116

1 U. S. Department of Energy Albuquerque Operations Office DOE AL ETD Attn: J. Lenhert Pennsylvania/H Street Albuquerque, NM 87116

\section{Corporations}

Westinghouse Savannah River Company Savannah River Technology Center Environmental Sciences Section P.O. Box 616 / 730-2B Aiken, SC 29802

1 Attn: Pete Zionkowski

1 Attr: Ahmet Seur

1 Attn: Thomas Gaughan

Coxporations (Continued)
3 Tech Reps, Inc.

Atth: Ralph D. Gruebel

5000 Marble NE

Albuquerque, NM 87110

\section{Libraries}

1 Thomas Branigan Library

Attn: D. Dresp

$106 \mathrm{~W}$. Hadley St.

Las Cruces, NM 88003

1 Government Publications

Zimmerman Library

University of New Mexico

$\begin{array}{lll} & \text { MS } & \text { O5g } \\ 1 & \overline{0719} & \text { 6621 } \\ 3 & 0719 & 6621 \\ 1 & 0986 & 2663 \\ 1 & 1159 & 9311 \\ 2 & 0719 & 6621 \\ 1 & 0756 & 6607 \\ 5 & 0899 & 4414 \\ 1 & 9018 & 8523-2 \\ 2 & 0619 & 12630\end{array}$

Library

C.V. Williams

R.A. Norman

G.J. Lockwood

T. Burford

G. Allen

Tecturical Library

Central Techrical

Files

Review \& Approval

Desk for DOE/OSiI 
This Page Intentionslly Left Blank

Dist-2 